\title{
A MULTIPHASE HARMONIC LOAD FLOW SOLUTION TECHNIQUE
}

by

WENYUAN XU

B. Eng., Xian Jiaotong University, 1982

M. Sc., University of Saskatchewan, 1985

A DISSERTATION SUBMITTED IN PARTIAL FULFILLMENT OF

THE REQUIREMENTS FOR THE DEGREE OF

DOCTOR OF PHILOSOPHY

IN

THE FACULTY OF GRADUATE STUDIES

DEPARTMENT OF ELECTRICAL ENGINEERING

We accept this thesis as conforming

to the required standard

THE UNIVERSITY OF BRITISH COLUMBIA

February 1990

(c) Wenyuan $\mathrm{Xu}, 1990$ 
In presenting this thesis in partial fulfilment of the requirements for an advanced degree at the University of British Columbia, I agree that the Library shall make it freely available for reference and study. I further agree that permission for extensive copying of this thesis for scholarly purposes may be granted by the head of my department or by his or her representatives. It is understood that copying or publication of this thesis for financial gain shall not be allowed without my written permission.

Department of Electrical Engineering

The University of British Columbia Vancouver, Canada

Date March 18,1990

DE-6 (2/88) 


\begin{abstract}
This thesis presents a comprehensive solution technique for power system harmonic analysis with unbalanced load flow conditions. It is based on multiphase modelling of the system in phase quantities.
\end{abstract}

Two of the most important features of this technique are the multiphase approach to the harmonic load flow problem, and the capability to add component nonlinearities easily. The first feature allows the technique to be used for either single-phase or threephase, and for either balanced or unbalanced harmonic analysis. The second feature allows the later addition of power electronic device models.

The technique is simple in concept. The nonlinear elements are first modelled as harmonic Norton equivalent circuits based on the network load flow conditions. These linear circuits are then included in the network solution with multiphase load flow constraints and network unbalances. Once the new load flow solutions are obtained, improved Norton equivalent circuits can be calculated, which in turn are used for improved network solutions. The entire solution scheme is therefore iterative, and stops when certain convergence criteria are met.

The unbalanced harmonics from nonlinear inductors, synchronous machines and static compensators with thyristor-controlled reactors are studied in this thesis. The convergence properties of the technique are investigated with test cases and theoretical analysis.

In addition to the harmonic load flow analysis, this technique can also be used as an improved initialization procedure for the Electromagnetic Transient Program (EMTP). 


\section{Table of Contents}

$\begin{array}{ll}\text { Abstract } & \text { ii }\end{array}$

List of Tables $\quad$ xi

List of Figures $\quad$ xiv

$\begin{array}{ll}\text { Acknowledgement } & \text { xv }\end{array}$

$\begin{array}{ll}\text { Nomenclature } & \text { xvi }\end{array}$

1 Introduction $\quad 1$

1.1 Power System Harmonics and Unbalances . . . . . . . . . . . . . 1

1.2 Techniques of Harmonic Analysis . . . . . . . . . . . . . . . . 3

1.2.1 Harmonic Solution: Time-domain Vs. Frequency-domain . . . . . 3

1.2.2 Harmonic Solution Viewed from Nonlinear Circuit Theory . . . . 4

1.2.3 Harmonic Solution Viewed from Power System Analysis . . . . . . 5

1.3 Power System Steady-state Analysis in Multiphase Framework . . . . . 7

1.4 The Purpose and Objectives of the Present Work . . . . . . . . . . 8

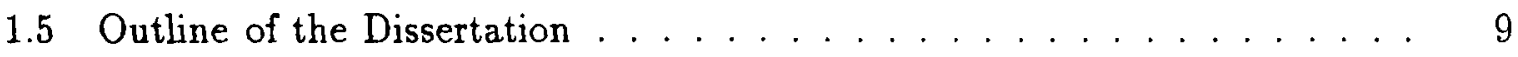


2.1 Static Compensator and Its Harmonics . . . . . . . . . . . . . 11

2.2 Principle of Harmonic Iteration $\ldots \ldots \ldots \ldots$

2.2 .1 The Basic Iterative Scheme $\ldots \ldots \ldots \ldots \ldots$

2.2 .2 Load Flow Constraints . . . . . . . . . . . . . . . . . . 14

2.2.3 Compensator System in Practice ... . . . . . . . . . 15

2.2.4 Summary of the Iterative Method . . . . . . . . . . . 17

2.3 Inclusion of Compensator Control Characteristics . . . . . . . . 17

2.3.1 Control Characteristics . . . . . . . . . . . . . . . . 18

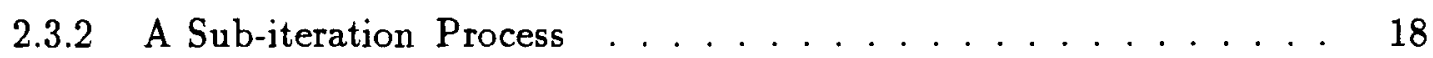

2.4 Discussion of Convergence Behaviour . . . . . . . . . . . . 20

2.5 Illustrative Examples . . . . . . . . . . . . . . . . . . 23

2.5.1 Comparison with Field Measurements . . . . . . . . . . . 24

2.5 .2 Harmonic Load Flow Solutions . . . . . . . . . . . . 24

2.5 .3 Convergence Behaviour . . . . . . . . . . . . . . 27

2.6 Conclusions . . . . . . . . . . . . . . . . . . . . 28

3 Multiphase Load Flow Solutions $\quad 30$

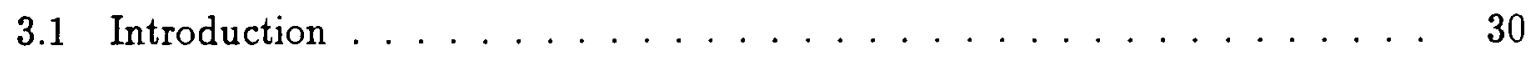

3.2 Modelling of Multiphase Network Components . . . . . . . . . . . 32 
3.2.1 Three-phase Synchronous Machines . . . . . . . . . . . . 32

3.2 .2 Multiphase Loads . . . . . . . . . . . . . . . . . 36

3.2 .3 Voltage and current sources $\ldots \ldots \ldots \ldots \ldots$

3.2 .4 Other Network Components . . . . . . . . . . . . . 39

3.3 Multiphase Load Flow Equations and Its Solutions $\ldots \ldots \ldots$

3.3.1 Formulation of Load Flow Equations . . . . . . . . . . . . 39

3.3.2 Solution of Load Flow Equations $\ldots \ldots \ldots \ldots$. . . . . . 41

3.4 Initialization of the Iterative Process $\ldots \ldots \ldots 43$

3.5 Frequency Scan Extension . . . . . . . . . . . . . . 45

3.5.1 Harmonic Models of Network Components . . . . . . . . . . 45

3.5 .2 Solution Technique . . . . . . . . . . . . . . . 46

3.6 Case Studies . . . . . . . . . . . . . . . . . . . . . 46

3.6.1 Field Measurement Comparisons . . . . . . . . . . . . 46

3.6.2 Unbalances in the B.C. Hydro Railway Case . . . . . . . . . 50

3.6.3 Frequency Scan Analysis of the TLR system . . . . . . . . . 51

3.6.4 Observations on the Convergence Behaviour ........ 52

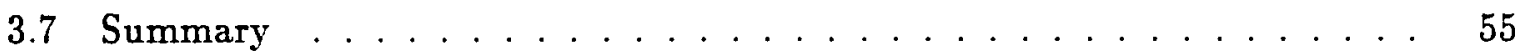

4 Multiphase Harmonic Load Flow Technique - The Basic Form 56

4.1 Frequency-domain Models of the Nonlinear Inductors . . . . . . . . 56 
4.2 Interface with the Multiphase Network Solution Process . . . . . . . . . 59

4.3 Test Results . . . . . . . . . . . . . . . . . . . . . 61

4.3.1 Comparison with Field Test Results . . . . . . . . . . . . 61

4.3.2 Comparison with EMTP Simulation Results . . . . . . . . . 63

4.3.3 Analysis of Harmonic Unbalance . . . . . . . . . . . . . 65

4.3.4 Numerical Convergence of the Solution . . . . . . . . . . . 68

4.4 MHLF technique for EMTP Initialization . . . . . . . . . . 68

4.4 .1 General Considerations . . . . . . . . . . . . . . 68

4.4.2 Load Models in the EMTP Transient Simulation . . . . . . . . 70

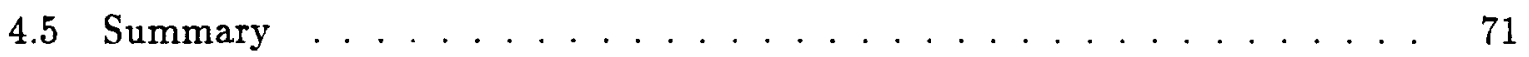

5 Modelling of Synchronous Machines as Harmonic Sources $\quad 73$

5.1 Harmonic Generation of Synchronous Machines . . . . . . . . 73

5.2 The MHLF Iterative Scheme . . . . . . . . . . . . . . . . 74

5.3 Machine Model with Frequency Conversion . . . . . . . . . . . 76

5.3.1 Harmonic Machine Model in DQO-coordinates . . . . . . . . 77

5.3.2 Harmonic Machine Model in ABC-coordinates . . . . . . . . 80

5.3 .3 Summary . . . . . . . . . . . . . . . . 83

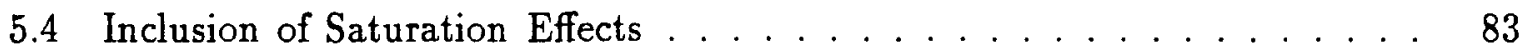

5.4.1 Saturation Effects in DQO-coordinates ........... . 84 


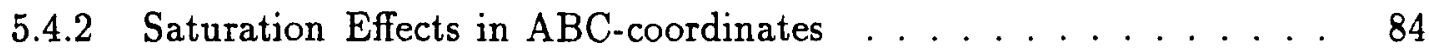

5.5 Case Studies . . . . . . . . . . . . . . . . . 85

5.5.1 Unbalanced Harmonic Solutions . . . . . . . . . . . . . 85

5.5.2 Comparison with Transient Simulations . . . . . . . . . 90

5.5.3 Comparison with Theoretical Results . . . . . . . . . . . . 91

5.5.4 Observations on the Convergence Behavior . . . . . . . . . 94

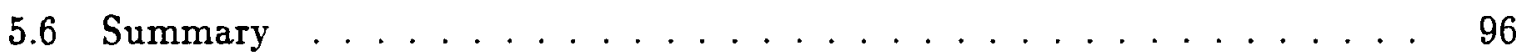

6 Unbalanced Harmonic Analysis of Static Compensators 97

6.1 Frequency-domain Model of Thyristor-Controlled Reactor . . . . . . . . 97

6.1.1 Harmonic Norton Equivalent Circuit of TCR . . . . . . . . 97

6.1.2 Considerations on the Practical Compensator Systems . . . . . 99

6.2 Interfacing with the MHLF Process $\ldots \ldots \ldots . \ldots \ldots 9$

6.3 Simulation of Static Compensator Control Characteristics . . . . . . . . 100

6.3.1 Control Characteristics in Multiphase Setting . . . . . . . 100

6.3.2 Conduction Angle Adjustment Scheme . . . . . . . . . . . . 101

6.4 Convergence Properties . . . . . . . . . . . . . . . . . . . . . 104

6.5 Case Studies and Applications . . . . . . . . . . . . 105

6.5.1 Field Measurement Comparisons . . . . . . . . . . . . . . 105

6.5.2 Investigation of Non-characteristic Harmonics . . . . . . . 105 
6.5.3 Harmonic Load Flow Solutions . . . . . . . . . . . . . . . . 111

6.5 .4 Convergence Behaviour . . . . . . . . . . . . . . . . 114

6.6 Summary . . . . . . . . . . . . . . . . . . . 116

7 Convergence Analysis of the MHLF Technique 118

7.1 General Considerations . . . . . . . . . . . . . . . . . . 118

7.2 A Simple Case: Static Network . . . . . . . . . . . . . . . . . . 119

7.2 .1 A Convergence Theorem . . . . . . . . . . . . . . . . 119

7.2.2 Analysis of the Basic Iterative Scheme . . . . . . . . . 121

7.2.3 Cases with More Than One Nonlinear Elements . . . . . . . . . 124

7.3 General Case: Dynamic Network . . . . . . . . . . . . . . . 125

7.3.1 Contraction Mappings in Harmonic Space . . . . . . . . . 126

7.3.2 Application 1: Nonlinear Inductor and Harmonic Resonance . . 128

7.3.3 Application 2: Thyristor-controlled Reactors . . . . . . . . 131

7.3.4 Application 3: Synchronous Machines . . . . . . . . . . . . 132

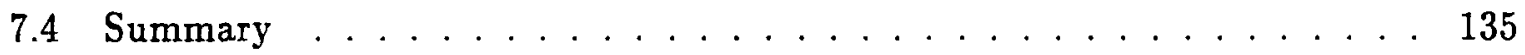

8 Conclusions $\quad 137$

$\begin{array}{lr}\text { References } & \mathbf{1 4 0}\end{array}$

$\begin{array}{lr}\text { A DQO-machine Model with Saturation } & 145\end{array}$ 
A.1 Flux Linkage Equation with Saturation . . . . . . . . . . . 145

A.2 Machine Model in DQO-coordinates . . . . . . . . . . . . 148

A.3 A Subiteration Process . . . . . . . . . . . . . . . 150

B Proof of Convergence Theorems 152

C Multiple Solutions in Unbalanced Load Flow Analysis 156

$\begin{array}{ll}\text { D Equivalent Circuit Models of Nonlinear Elements } & 161\end{array}$

E Selected Test System Data 162 


\section{List of Tables}

2.1 Bus voltage harmonics. . . . . . . . . . . . . . . . . 26

2.2 Static compensator current injections and conduction angles. . . . . 27

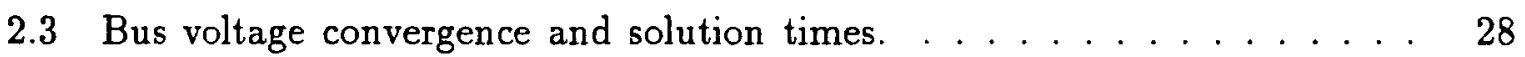

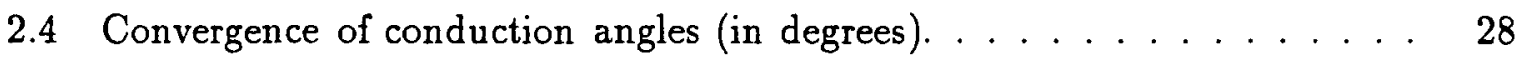

3.1 Comparison between proposed and existing load flow techniques . . . . 42

3.2 Percentage unbalance of motor currents. . . . . . . . . . 50

3.3 TLR percentage voltage unbalance with power factors. . . . . . . . 51

3.4 Convergence behaviour of the multiphase load flow technique. . . . . 54

4.1 Harmonic contents of the unbalanced test case. . . . . . . . . . 67

4.2 Convergence behaviour. . . . . . . . . . . . . . . 68

5.1 Third harmonic current caused by fundamental frequency voltage and current unbalance for five test machines. . . . . . . . . . . 86

5.2 Harmonic profiles of two synchronous machines. . . . . . . . 88

5.3 Third harmonic voltages and currents with Y-g and $\Delta$ connection. . . 88

5.4 Voltage and current harmonics with and without saturation. . . . . 89

5.5 Machine 1 voltages with other harmonic sources. . . . . . . . . 90 
5.6 Values of negative sequence impedance. . . . . . . . . . . 93

5.7 Convergence behavior of the MHLF technique. . . . . . . . . . 95

6.1 Convergence behaviour of the MHLF technique. . . . . . . . 115

D.1 List of nonlinear elements and their equivalent circuits . . . . . . . 161

E.1 List of test cases. . . . . . . . . . . . . . . . . . . . 162

E.2 List of test cases (continue from previous table) . . . . . . . 163

E.3 Transformer short circuit test data. . . . . . . . . . . . . . . 163

E.4 Transmission line and equivalent source data. . . . . . . . . . . . 164

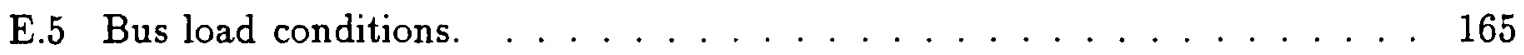

E.6 Data of static compensator components and filters. . . . . . . 166

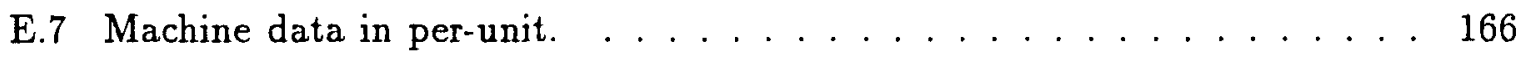




\section{List of Figures}

2.1 Thyristor-controlled reactor and waveforms. ............ 13

2.2 The structure of a practical static compensator. . . . . . . . . . 16

2.3 V-I characteristic of the static compensator. . . . . . . . . . . . 19

2.4 Solution for conduction angle. . . . . . . . . . . . . 20

2.5 Divergence of the iterative process. . . . . . . . . . . . . 21

2.6 Improved iterative process. . . . . . . . . . . . . . . 22

2.7 Iteration of harmonic component. . . . . . . . . . . . . . 23

2.8 Waveform comparison with field measurements. . . . . . . . . . . 25

3.1 Branch models for multiphase load flow analysis. . . . . . . . . . . 35

3.2 The B.C. Hydro TLR area network with single-phase railway load. . . . 47

3.3 TLR bus voltage unbalance as a function of railway load. . . . . . . . 48

3.4 Bus current unbalance as a function of TLR bus voltage unbalance. . . . 49

3.5 Frequency scan of the TLR system. . . . . . . . . . . . . . . 52

3.6 Single-phase diagram of test system $3 . \ldots \ldots$. . . . . . . . . . . 53

4.1 Characteristics of a nonlinear inductor. . . . . . . . . . . . 57

4.2 Norton equivalent circuit of a nonlinear inductor. . . . . . . . . . 58 
4.3 Flow chart of the MHLF technique. . . . . . . . . . . . . . 62

4.4 Single-phase diagram of test system CEMIG. . . . . . . . . . . . . 63

4.5 Comparison between MHLF and field test results. . . . . . . . . . . 64

4.6 Comparison between MHLF and EMTP results. . . . . . . . . . . 66

4.7 Analysis of harmonic unbalances. . . . . . . . . . . . . . . . . 69

5.1 A synchronous machine model with nonlinear effects included. . . . . . 75

5.2 Process of frequency conversion. . . . . . . . . . . . . . . . 82

5.3 One machine test system. . . . . . . . . . . . . . . 87

5.4 One machine test system with other harmonic sources. . . . . . . . 90

5.5 Comparison of waveforms from EMTP and MHLF. . . . . . . . . . 92

5.6 Two tests to determine machine negative sequence impedance. . . . . . . 93

5.7 A multimachine test system. . . . . . . . . . . . . . . . . . 95

6.1 TCR model for multiphase harmonic analysis. . . . . . . . . . . . 98

6.2 Solution of conduction angles. . . . . . . . . . . . . . 102

6.3 A typical static compensator. . . . . . . . . . . . . . 106

6.4 Harmonic current injection as a function of the compensator bus voltage

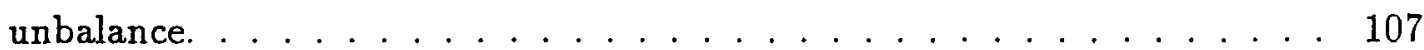

6.5 Harmonic current injection as a function of conduction angle. . . . . 108

6.6 Harmonic current injection as a function of firing asymmetry. . . . . . . . 109 
6.7 Harmonic current injection as a function of TCR reactor unbalance. . . . 110

6.8 Static compensator bus voltage harmonic spectrum. . . . . . . . . . . 112

6.9 TCR bus voltage harmonic spectrum. . . . . . . . . . . . . 113

6.10 Compensator current injection spectrum. . . . . . . . . . . 114

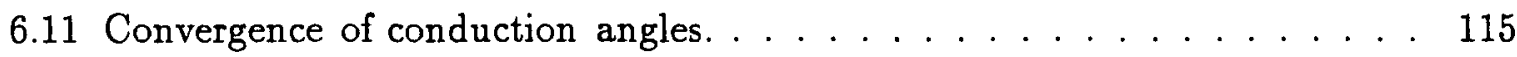

7.1 Iterative solution process of Equation $(7.1) \ldots \ldots \ldots$

7.2 Harmonic iteration of a simple resistive network. . . . . . . . 121

7.3 Convergence comparison between two modelling techniques. . . . . . 124

$7.4 \mathrm{~A}$ simple nonlinear inductor circuit. . . . . . . . . . . . . . 129

7.5 TCR $\lambda-i$ characteristics. . . . . . . . . . . . 132

7.6 A synchronous machine connected to a network (single-line diagram). . . 133

A.1 Normalized saturation curve of a synchronous machine. . . . . . . 145

C.1 Load arrangement that causes multiple solutions. . . . . . . . . . 157

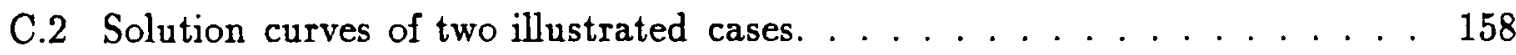

C.3 Interpretation of two solutions when $\beta=1 \ldots \ldots \ldots 160$

E.1 19 bus TransAlta Utilities Corporation test system. . . . . . . . 165 


\section{Acknowledgement}

I would like to express my sincere thanks and gratitude to my supervisors, Dr. Hermann W. Dommel and Dr. Jose R. Marti, for their continued interest, encouragement and guidance during the course of this research.

I wish to extend my gratitude to the engineers of British Columbia Hydro and Power Authority and TransAlta Utilities Corporation. In particular, I would like to thank Dr. K.C. Lee for revising this manuscript, Mr. T. Martinich, Mr. B. Hughes and Mr. I. Lu for providing the field test data used in this study.

I am also deeply grateful to my wife Amanda (Xiaomin) for her help in many aspects during this project, but most of all for her love and patience.

Thanks are due to Mr. G. Peterson for proof reading the thesis, to Mr. R. Ross for his tireless help in the usage of the computer facilities, and to my graduate fellows for their valuable comments and encouragement.

The financial assistance from British Columbia Hydro and Power Authority is gratefully acknowledged.

Finally, to those families and friends who cared, I offer my warmest thanks. 


\section{Nomenclature}

The following conventions will be followed in this dissertation.

- Bold-faced symbols indicate phasors.

- Bracketed symbols represent matrices and vectors.

- Lower case italic symbols refer to time functions.

- Symbols followed by $(h)$, such as $Y_{a b c}(h)$, indicate functions of harmonic order $h$.

Whenever necessary, deviations from these conventions will be indicated explicitly. 


\section{Chapter 1}

\section{Introduction}

\subsection{Power System Harmonics and Unbalances}

The concern about voltage and current harmonic distortion in power systems has resulted from the use of nonlinear devices. The concept of harmonics refers to the distortion of sinusoidal waveforms. These distorted waveforms are usually periodic. With Fourier analysis, they can be expressed as a fundamental frequency sinusoidal component and other higher order frequency sinusoidal components - the harmonics.

Power system harmonics are not new phenomena. As an unwelcome by-product, they have been present almost since the inception of $\mathrm{AC}$ networks. In the early power systems, nonlinear devices were rare. Harmonic distortion was not a concern in most cases. However, this situation has changed with the increased use of power electronic devices and economic design of ferromagnetic components. Waveform distortion in modern power systems is no longer negligible. It is estimated that 5 percent electric energy consumed today passes through power convertors. This amount is projected to increase to 40 percent shortly after the turn of the century [1]. In view of the already substantial cases of harmonic distortion, the control of harmonic pollution has become a major recent industrial concern.

The effects of harmonic distortion are numerous [1]. The excessive voltage harmonics

may cause insulation failure and shunt capacitors damage. When the harmonic currents 
are large and travel in parallel with communication circuits, telephone interference may occur. Transmission line losses are also increased by the presence of harmonic currents. Furthermore, watthour meter error is often a concern as well.

While the unbalance of three-phase power systems is itself a problem, it adds complications to the harmonic distortion. The fact is that harmonics are more sensitive to the network unbalance: even for small deviation from balanced conditions at power frequency, it has been noted that harmonic unbalance can be significant and it increases with harmonic orders $[2,3]$. In many cases, unbalanced operations can either cause the generation of extra so-called noncharacteristic harmonics ${ }^{1}$ or turn a linear component into a harmonic-producing nonlinear component (synchronous machine is an example). In other cases such as telephone interference, the nature of the problems requires an unbalanced harmonic analysis.

In normal operations, three-phase power systems are always kept as balanced as possible. However, the real situations such as the presence of untransposed transmission lines, single-phase loads, and other unbalanced sources render perfect balance impossible. Taking into consideration the sensitivity of harmonics to system unbalance, there is a strong need to include network unbalances into any harmonic investigation.

The ultimate objective to study harmonics is to reduce their effect on power systems and their customers. In recent years, this objective has stimulated considerable research on harmonic techniques, both in the area of hardware and software.

\footnotetext{
${ }^{1}$ Noncharacteristic harmonics refer to those harmonics which are ideally cancelled out by each other with special arrangement of the harmonic-producing elements. Such cancellations cannot be obtained if the operating conditions are unbalanced.
} 


\subsection{Techniques of Harmonic Analysis}

An understanding of harmonic problems requires the coordinated development of measurement and computer simulation techniques. While the measurement techniques are making much progress with the advance of signal processing techniques, the computer simulations can hardly meet the increasing requirements of harmonic analysis. However, the well-recognized benefits of simulation techniques have been motivating the development of harmonic analysis software to study both power systems and general electric circuits.

\subsubsection{Harmonic Solution: Time-domain Vs. Frequency-domain}

The central problem of harmonic analysis is to find the steady-state solutions of a nonlinear dynamic network with (single frequency) periodic excitations. The resultant steadystate waveforms can either be expressed as periodic time functions or as summations of harmonic phasors. Depending on the way of formulating the problem, harmonic solution techniques fall into two general categories, time-domain methods and frequency-domain methods.

As a principle, time-domain formulations have the advantage of dealing directly with real physical phenomena. This property greatly facilitates the modelling of nonlinear elements which are the sources of harmonics.

Frequency-domain formulations are originated from abstract mathematical transformations. However, its physical meaning in linear systems is widely accepted. For a linear system, the steady-state response possesses a linear relationship with sinusoidal excitations. This feature has made the frequency-domain approaches very attractive for steady-state linear circuit analysis. 
Thus the harmonic solution methods face a dilemma: on one hand, harmonics are steady-state phenomena that can easily be dealt with in frequency-domain analysis, on the other hand, they are related to component nonlinearities which are easier to be included in time-domain analysis.

\subsubsection{Harmonic Solution Viewed from Nonlinear Circuit Theory}

Nonlinear elements exist not only in power networks, but also in many other electric circuits. A wider view of the problem leads to the topic of nonlinear circuit analysis. As a matter of fact, there has been a sustained attempt to develop a technique for calculating the steady-state waveforms of general nonlinear circuits. Typical progress on the subject is summarized as follows:

1. Transient Simulation Method. This is the simple numerical integration method which is usually used for transient simulations. The steady-state is obtained when the transients have died out. As a time-domain approach, the method is very general and easy to model nonlinearities. But it is prohibitively expensive for lightly damped circuits where a long simulation time is needed for these transients to die out.

2. Shooting Method. This type of approach solves the problem by finding an initial state $x_{0}$ such that the network state starting from $x_{0}$ is periodic or has no transients. Aprill and Trick proposed this idea and applied the Newton-Raphson method for its solution [4]. Gradient methods [5] and extrapolation methods [6] were introduced later to solve the same problem. The shooting method is a time-domain approach. It shares the similar characters of the time-domain formulation. ${ }^{2}$

\footnotetext{
${ }^{2}$ Strict mathematical analysis indicates that the transient simulation is a shooting method solved with a simple iterative technique [4].
} 
3. Harmonic Balance Method. This type of approach assumes that the solution consists of a series of harmonic phasors. By substituting them into the differential equations of the circuit and equating the terms of same frequencies, a set of algebraic equations is formed. In its original formulation, Baily used an optimization algorithm to find the phasors [7]. This method is further improved by Ushida and Chua with the utilization of the Newton-Raphson algorithm [8]. The harmonic balance method is clearly a frequency-domain approach. It has the difficulty to model nonlinear elements. But this method can be simple and fast when only a few nonlinear elements are present.

In spite of these developments, none of the methods has been widely accepted as a general harmonic solution technique. A few of the methods may be used for power system harmonic analysis. But many improvements are needed in order to include the unique features of power networks.

\subsubsection{Harmonic Solution Viewed from Power System Analysis}

Compared with other circuits, the unique features of power networks include the active and reactive power constraints, the relative dominance of linear elements and the control characteristics of nonlinear elements. Accordingly, power system analysts have developed their own approaches to harmonic analysis, independent of the nonlinear circuit theory.

Time-domain simulations, such as the one provided by the Electromagnetic Transients Program (EMTP) [9], have been used to solve some harmonic problems [10]. With the same idea, Kitchin developed a numerical integration program dedicated for power system harmonic analysis [11]. As mentioned before, these methods are flexible to model nonlinearities but time consuming for lightly damped networks. If load flow constraints are to be included, they exhibit extra limitations. 
The frequency scan technique is a useful tool for harmonic analysis. However, it can hardly be considered as a harmonic solution technique. In this approach, nonlinear elements are simply treated as known harmonic current sources whose values are either set to 1 per-unit or determined from theoretical analysis of the elements. Node voltages are found by solving node voltage equations of different frequencies. Many programs have been developed to perform this function [3]. Among them, the option provided by the EMTP [12] might be the most popular.

Harmonic iteration is an advancement of the frequency scan. In this case, the current sources representing nonlinear elements are determined according to the network voltages. With these current sources, improved node voltages are computed, which in turn are used to determine improved current sources. This iterative process is continued until certain criteria are met. This idea was first applied by Yacamini and de Oliveira to analyze convertor bridges [13]. It has also been independently used by Dommel, Yan and Shi for the analysis of nonlinear inductors [14]. Although neither load flow constraints nor other control characteristics have been considered in these developments, the simplicity of the idea makes it a promising candidate for further exploration.

The most important advancement in harmonic load flow analysis is probably due to $\mathrm{Xia}$ and Heydt [15]. In their pioneering HARMFLO program, a system of algebraic equations with harmonic voltages as unknowns was established by the principle of harmonic balance. It is then solved with the Newton-Raphson method. This program was further extended to include HVDC subnetworks [16]. In addition to the inclusion of load flow constraints, HARMFLO has the advantage of fast convergence if a proper starting point is used. As a common disadvantage of the Newton-Raphson method, the computation of the Jacobian matrix may be difficult. This is unfortunately the case in the HARMFLO technique. There are also some difficulties in extending the technique to multiphase 
harmonic analysis.

In the framework of nonlinear circuit theory, both harmonic iteration and HARMFLO techniques belong to the harmonic balance method. It is interesting to note that there is no power system harmonic technique that corresponds to the shooting method. One apparent difficulty of the shooting method is the inclusion of power constraints. Nevertheless, it is still possible that this method may find its use for power system harmonic analysis in the future.

\subsection{Power System Steady-state Analysis in Multiphase Framework}

Traditionally, the analysis of unbalanced power systems has been limited to transient and dynamic studies. For steady-state analysis, balanced operation is normally assumed. In view of the increased existence of many significant unbalance sources, the necessity of three-phase steady-state analysis is obvious, needless to mention the harmonic situations. To analyze these conditions, the development of a general purpose multiphase load flow technique is warranted.

The concept of three-phase load flow analysis was first introduced by El-Abiad and Tarsi [17]. In their work, the importance to develop three-phase component models was emphasized and a generator model was proposed accordingly. Since then, much progress is reported. This includes the applications of decoupled techniques [18] and graph theory [19]. A three-phase load flow option was also included in the EMTP [12]. In their book, Arrillaga, Arnold and Harker provide a good summary on the current three-phase load flow techniques [18].

In spite of these latest developments, which are mostly concerned with numerical solution aspects, many fundamental concepts remain untouched. For example: 
- What are the three-phase or multiphase load models?

- How to formulate load flow equations that can model all possible component connections in the multiphase environment?

- How to find a starting point for the iterative solution process?

These problems clearly suggest that a simple extension of the single-phase load flow techniques cannot satisfy the need of unbalanced load flow analysis. A comprehensive approach must be developed.

Partly due to this situation, no technique has been developed for multiphase harmonic load flow analysis.

\subsection{The Purpose and Objectives of the Present Work}

It is the purpose of the work in this dissertation to develop a general purpose Multiphase Harmonic Load Flow (MHLF) technique for power system analysis. In view of the current situation in harmonic analysis, the characteristics of power systems and the prospect in nonlinear device applications, the proposed technique shall meet the following requirements:

1. As a load flow technique, it can be used for either regular (i.e. fundamental frequency) load flow or harmonic load flow analysis. Both balanced and unbalanced conditions with any number of coupled phases can be studied.

2. As a harmonic analysis technique, it shall provide a wide range of application purposes. Namely, it can be used as a simple frequency scan technique on one end, or as a comprehensive harmonic load flow technique on the other. 
3. Load flow constraints and other control characteristics can be included upon user's choice. This technique shall also take into account the property of linear element dominance in power networks.

4. With respect to the prospective use of power electronic devices, the technique must possess extensibility and flexibility. More specifically, the user can easily extend the program and data structure to include the nonlinearities of his choice.

5. This technique can also be used to initialize time-domain transient simulations such as to provide improved initializations for the EMTP.

6. The MHLF technique shall be simple and accurate in both concept and mathematical formulation. It must be computationally efficient as well.

The proposed multiphase harmonic load flow technique is based on the principle of harmonic balance. It is therefore a frequency-domain method. With the adoption of the harmonic iteration idea, however, the technique allows time-domain analysis of nonlinear elements. Thus the advantages of both time-domain and frequency-domain analysis are included, as will be explained in the dissertation.

\subsection{Outline of the Dissertation}

In view of the current achievements in the methods for harmonic solutions and for multiphase steady-state analysis of power networks, some more basic problems must be solved before the MHLF technique can be considered. On one hand, the existing harmonic techniques shall be improved so that they can be extended to multiphase networks. On the other hand, the current multiphase load flow techniques need thorough investigation and development. The strategy adopted in this work is to develop at first a new single-phase harmonic load flow technique that can easily be advanced into a multiphase framework. 
A multiphase fundamental frequency load flow technique is then proposed as a basis for the advancement. Accordingly, this research is conducted as follows:

- The idea of the MHLF technique is first tested by developing a single-phase version of the technique. Balanced harmonic load flows of static compensators with thyristor-controlled reactors are analyzed. These investigations are presented in Chapter 2.

- The systematic development of the MHLF technique starts with Chapter 3 with the proposition of a general purpose multiphase load flow technique. This technique is then extended with frequency scan capability. This forms the basis of the MHLF technique.

- The general solution procedures of the MHLF technique is outlined in Chapter 4. This is illustrated by the inclusion of nonlinear inductors in the analysis.

- The MHLF technique is further expanded to include synchronous machines as harmonic sources. The responses of machines to unbalanced and harmonic conditions are investigated in Chapter 5.

- Unbalanced harmonic load flows of static compensators are analyzed in Chapter 6 using the MHLF technique. The compensator control characteristics are modelled and the generation of noncharacteristic harmonics is studied.

- The MHLF technique is an iterative solution technique. In the final development, convergence properties of the technique are discussed. The results are reported in Chapter 7. 


\section{Chapter 2}

\section{Single-Phase Harmonic Analysis of Static Compensators}

As indicated in Chapter 1, the existing harmonic analysis techniques are either unsuitable for multiphase extension or incomplete in load flow considerations. Therefore, in order to develop a full multiphase program for harmonic analysis, it is necessary to establish a new harmonic solution scheme at first. This scheme is proposed and tested for the singlephase case in this chapter. A static compensator is used as the source of harmonics to explain the scheme.

\subsection{Static Compensator and Its Harmonics}

The static compensator with thyristor-controlled reactors (TCR) has been known as an effective and reliable means for power system voltage regulation. This device is essentially a variable reactor that can adjust its reactive power consumption. The adjustment is realized through the firing angles of thyristors. The thyristors normally operate in partial conducting states, and thereby produce harmonics [20].

These harmonics need to be calculated so that harmonic mitigative measures can be designed. For thyristor operation with fixed firing angles, the magnitudes of the TCR harmonics are reasonably well known $[20]$ and the resultant harmonic distributions can easily be found with existing techniques. For normal operation of the compensator, however, these harmonics depend critically on its control characteristics, which make the firing angles dependent on the network load flow conditions and harmonic voltage 
distributions.

In power electronic devices, it is common that the firing angles and the generation of harmonics depend on the load flow conditions. The proposed solution method must be able to take such properties into account. With the assumption of balanced operating conditions, a new single-phase harmonic load flow technique is developed for the static compensators.

\subsection{Principle of Harmonic Iteration}

The basic element of the static compensator is the thyristor-controlled reactor. It is a reactor in series with anti-parallel thyristor valves, as shown in Figure 2.1. The valves conduct on alternate half-cycles of the supply frequency, for durations which depend on their firing angles $\alpha$. The operating range of firing angles goes from no conduction at $\alpha=180^{\circ}$ to full conduction at $\alpha=90^{\circ}$. The duration of conduction is defined by the conduction angle

$$
\sigma=2\left(180^{\circ}-\alpha\right)
$$

If $\sigma$ is less than $180^{\circ}$, harmonic currents are generated, as shown in Figure 2.1. To explain the harmonic iteration scheme for obtaining harmonic solutions, the TCR operation with known conduction angles is considered first.

\subsubsection{The Basic Iterative Scheme}

In general, the voltages appearing across the TCR will be distorted with harmonics,

$$
v(t)=\sum_{h=1}^{n} \sqrt{2}\left|\mathbf{V}_{h}\right| \cos \left(h \omega t+\phi_{h}\right),
$$

where $n$ is the highest harmonic order of interest. With the valve fired at $t_{f}$, the current through the reactor during one half of a cycle is determined by the following equations:

$$
L(d i / d t)=v(t), \quad i\left(t_{f}\right)=0
$$



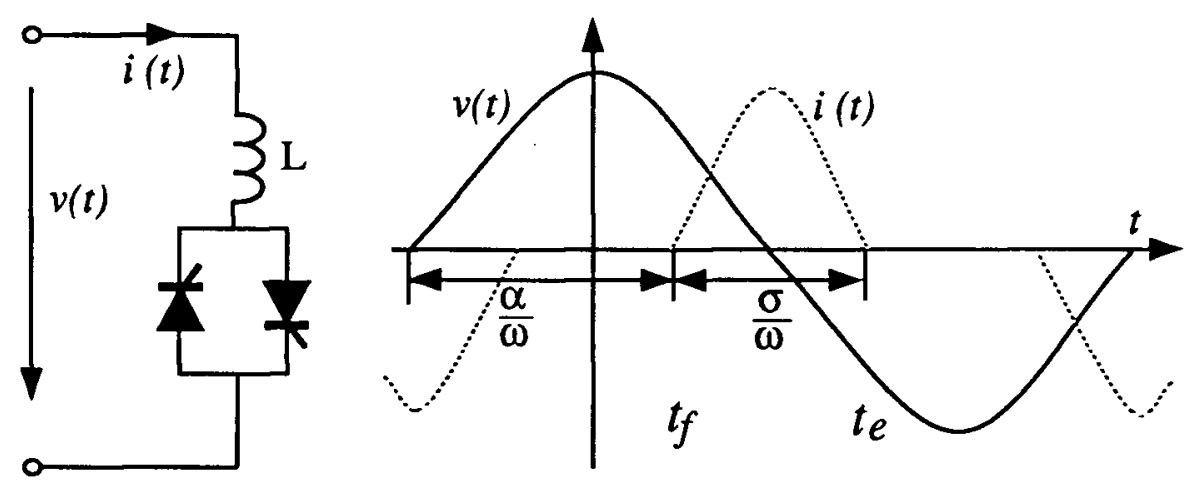

Figure 2.1: Thyristor-controlled reactor and waveforms.

or

$$
i(t)=\left\{\begin{array}{l}
\sum_{h=1}^{n} \sqrt{2}\left|\mathbf{V}_{h}\right|(h \omega L)^{-1}\left[\sin \left(h \omega t+\phi_{h}\right)-\sin \left(h \omega t_{f}+\phi_{h}\right)\right], \quad t_{f} \leq t \leq t_{e}, \\
0, \quad 0<t<t_{f} \text { and } t_{e}<t<T / 2
\end{array}\right.
$$

where $t_{f}$ is the instant of firing, $t_{e}$ that of extinction, and $\mathrm{T}$ the cycle period at fundamental frequency. For the second half cycle, the currents will reverse in sign (Figure 2.1). To obtain the current phasors as a function of the voltage phasors, Fourier analysis is required. Since the closed-form Fourier analysis of Eq. (2.3) is complicated due to discontinuous conduction, the distorted current is generated point-by-point from the given voltage with Eq. (2.3) and then analyzed with discrete Fourier analysis. This produces the harmonic content expressed by

$$
i_{\text {harmonic }}(t)=\sum_{h=1}^{n} \sqrt{2}\left|\mathbf{I}_{h}\right| \cos \left(h \omega t+\theta_{h}\right) .
$$

In other words, for given voltages at the TCR terminal, the corresponding current phasors can always be computed with this simple (time-domain) steady-state numerical process. This relationship is symbolically expressed as a nonlinear function

$$
\left[\mathbf{I}_{1}, \mathbf{I}_{H}\right]=N\left(\mathbf{V}_{1}, \mathbf{V}_{H}\right),
$$


where $\mathbf{I}_{1}$ and $\mathbf{V}_{1}$ are the fundamental frequency current and voltage phasors, and $\mathbf{I}_{\boldsymbol{H}}$ and $\mathrm{V}_{H}$ are the harmonic current and voltage phasors, respectively. This approach can be modified to include the reactor resistances as well.

With known harmonic currents, the nonlinear TCR can be replaced by voltagedependent current sources at fundamental and harmonic frequencies. The network then becomes a linear network, and the node voltages at each harmonic are then easily found by a frequency scan process, namely to solve a system of nodal equations of the form

$$
\left[Y_{h}\right]\left[\mathbf{V}_{h}\right]=\left[\mathbf{I}_{h}\right], \quad h=1, \ldots, n,
$$

where the nonlinear TCR effects are represented as currents in the vector $\left[\mathbf{I}_{h}\right]$. Once the voltages have been found for the fundamental and harmonic frequencies, an improved equivalent current source can then be calculated from Eq. (2.5), which in turn is used to compute improved voltages. This is the harmonic iteration process. It is continued until the changes of TCR voltages or currents between two successive iterations are within certain convergence limits.

\subsubsection{Load Flow Constraints}

To include load flow features, Eq. (2.6) at fundamental frequency $(h=1)$ must be modified to take the usual load flow constraints of constant active and reactive power $(P, Q-$ bus), of constant active power and voltage magnitude ( $\mathrm{P}, \mathrm{V}$-bus) and other constraints (transformer control, area interchange control, etc.) into account. In effect, Eq. (2.6) at $h=1$ becomes the normal load flow equations, except that TCR's appear as additional current injections. In this study, the Newton-Raphson method in the power equation form with rectangular coordinates [21] was chosen to solve the load flow equations at fundamental frequency, which can be symbolically expressed in the form

$$
\left[\mathbf{V}_{1}\right]=F\left(\left[\mathbf{I}_{1}\right]\right),
$$


where $\left[V_{1}\right]$ is the fundamental frequency bus voltage vector, and $\left[I_{1}\right]$ the fundamental frequency current injection vector representing the TCR's. Note that Eq. (2.7) assumes that the usual $|\mathrm{V}|, \mathrm{P}$ and $\mathrm{Q}$ constraints are specified for the fundamental frequency only.

At harmonic frequencies, generators are represented as equivalent impedances in Eq. (2.6). It is also assumed that the PQ loads are presented in a similar form. Reference [22] recommends:

$$
\begin{gathered}
Z_{\text {generator-h }}=j h X_{\text {negative }} \\
Z_{\text {load-h }}=\left(R_{\text {series }}+j X_{\text {oeries }}\right) / / j X_{\text {parallel }}
\end{gathered}
$$

with

$$
\begin{aligned}
R_{\text {series }} & =\left|V_{1}\right|_{\text {rated }}^{2} / P_{1} \\
X_{\text {series }} & =0.073 h R_{\text {series }}, \\
X_{\text {parallel }} & =h R_{\text {series }} /\left[6.7\left(Q_{1} / P_{1}\right)-0.74\right]
\end{aligned}
$$

where $h$ is the harmonic order, and $X_{\text {negative }}$ the negative sequence reactance of the generator at fundamental frequency. Transmission lines are represented as exact equivalent $\pi$-circuits at each harmonic frequency [12]. All these equivalent impedances are then used to build the harmonic $\left[Y_{h}\right]$ matrices. Since these matrices remain constant for all iteration steps, they are only triangularized once outside the iteration loop.

\subsubsection{Compensator System in Practice}

A practical compensator system, such as the one shown in Figure 2.2, is more complicated than described so far. First of all, the effects of TCR delta connections must be included in Eq. (2.5). This is achieved by eliminating the triple harmonics and shifting phase angles for each harmonic component in the equation. The phase-shifting effect of the 
transformer connections must be represented as well, which can be done with a phaseshifter model [23]. For the three-winding transformer of Figure 2.2, the arm of the well known star circuit on the delta side would be represented by such a model. The threewinding transformer connection of Figure 2.2 leads to the cancellation of the 5 th and 7 th harmonics [20], which is useful to reduce harmonic distortions.

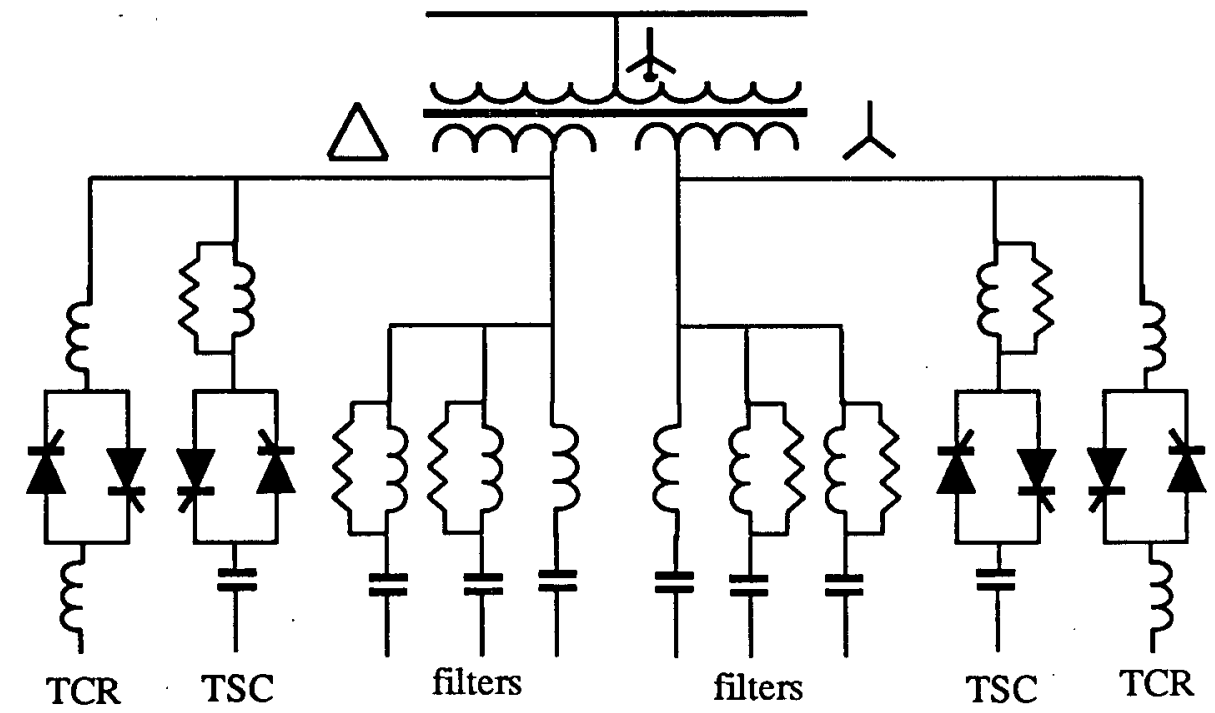

(a) Static compensator structure (single-phase diagram)

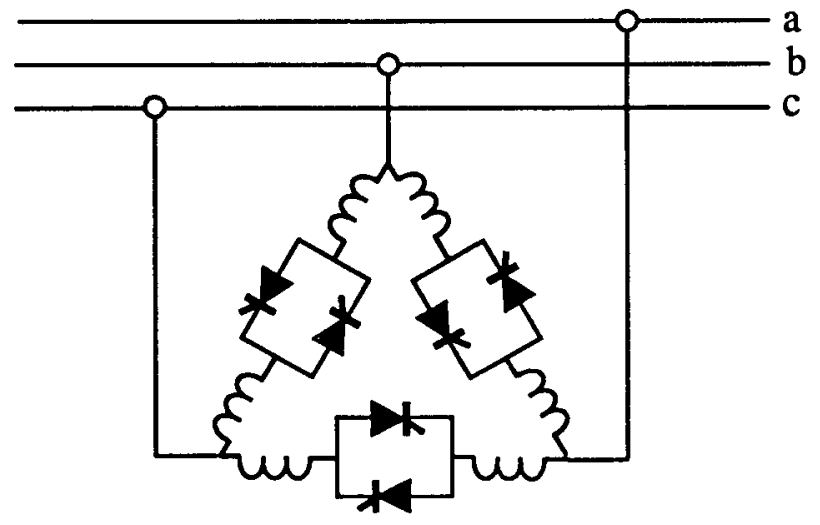

(b) TCR delta connection

Figure 2.2: The structure of a practical static compensator. 
It shall be noted that phase-shifter models are unsymmetric. Their inclusion leads to an unsymmetric $\left[Y_{h}\right]$ matrix. However, if the network is represented in the multiphase frame, there is no such problem because the phase shift can be realized by proper node assignments rather than by a phase angle rotation, as proposed in [12].

\subsubsection{Summary of the Iterative Method}

The iterative process can now be summarized in the following form of three equation sets:

$$
\begin{aligned}
\text { Nonlinear TCR elements: } & {\left[\mathbf{I}_{1}, \mathbf{I}_{H}\right]=N\left(\left[\mathbf{V}_{\mathbf{1}}\right],\left[\mathbf{V}_{H}\right]\right) } \\
\text { Fundamental frequency load flow: } & {\left[\mathbf{V}_{1}\right]=F\left(\left[\mathbf{I}_{1}\right]\right) } \\
\text { Harmonic frequency scan: } & {\left[\mathbf{V}_{H}\right]=H\left(\left[\mathbf{I}_{H}\right]\right) }
\end{aligned}
$$

where $\left[\mathbf{I}_{1}\right],\left[\mathbf{I}_{H}\right]$ are the fundamental frequency and harmonic current phasor vectors, and $\left[\mathbf{V}_{1}\right],\left[\mathbf{V}_{H}\right]$ are the voltage phasor vectors, respectively. With the described iterative process, these three equation sets are solved as follows:

$$
\begin{aligned}
{\left[\mathbf{I}_{1}(J+1), \quad \mathbf{I}_{H}(J+1)\right] } & =N\left(\left[\mathbf{V}_{1}(J)\right], \quad\left[\mathbf{V}_{H}(J)\right]\right) \\
{\left[\mathbf{V}_{1}(J+1)\right] } & =F\left(\left[\mathbf{I}_{1}(J+1)\right]\right) \\
{\left[\mathbf{V}_{H}(J+1)\right] } & =H\left(\left[\mathbf{I}_{H}(J+1)\right]\right)
\end{aligned}
$$

where $\mathrm{J}$ indicates the iteration number.

\subsection{Inclusion of Compensator Control Characteristics}

For the purpose of voltage regulation, the reactive power generated or absorbed by the static compensator is automatically adjusted according to certain characteristics. The TCR conduction angle is therefore an unknown variable in the normal operation. A 
practical harmonic load flow technique must be able to calculate the conduction angles and the corresponding harmonic distributions under any given load flow condition.

\subsubsection{Control Characteristics}

The most common static compensator control characteristic is the linear voltage-current relationship shown in Figure $2.3[20]$. It is realized through the proper design of the measurement, control and firing units. In the normal steady state, the conduction angle is automatically adjusted by the controller such that

$$
\left|\mathbf{V}_{1}\right|=V_{s}+I_{r} k
$$

where

$V_{1}$ is the fundamental frequency voltage at the compensator bus,

$V_{a}$ is the voltage set point,

$I_{r}=\left|\mathbf{I}_{1}\right| \sin \left(\theta_{1}-\phi_{1}\right)$ is the reactive component of $\mathbf{I}_{1}$,

$k$ is the slope constant,

$\mathbf{I}_{1}$ is the compensator current injected into the network, and

$\phi_{1}, \theta_{1}$ are the phase angles of $V_{1}$ and $I_{1}$ respectively.

This is the compensator characteristic equation. The device operates at the intersection point of its control characteristic with the network characteristic, as shown in Figure 2.3. To include this constraint in the solution, a sub-iteration scheme is used.

\subsubsection{A Sub-iteration Process}

Assume that the bus voltages are known initially. For a given conduction angle, the fundamental frequency current phasor can be found from Eq. (2.5). These voltage and 


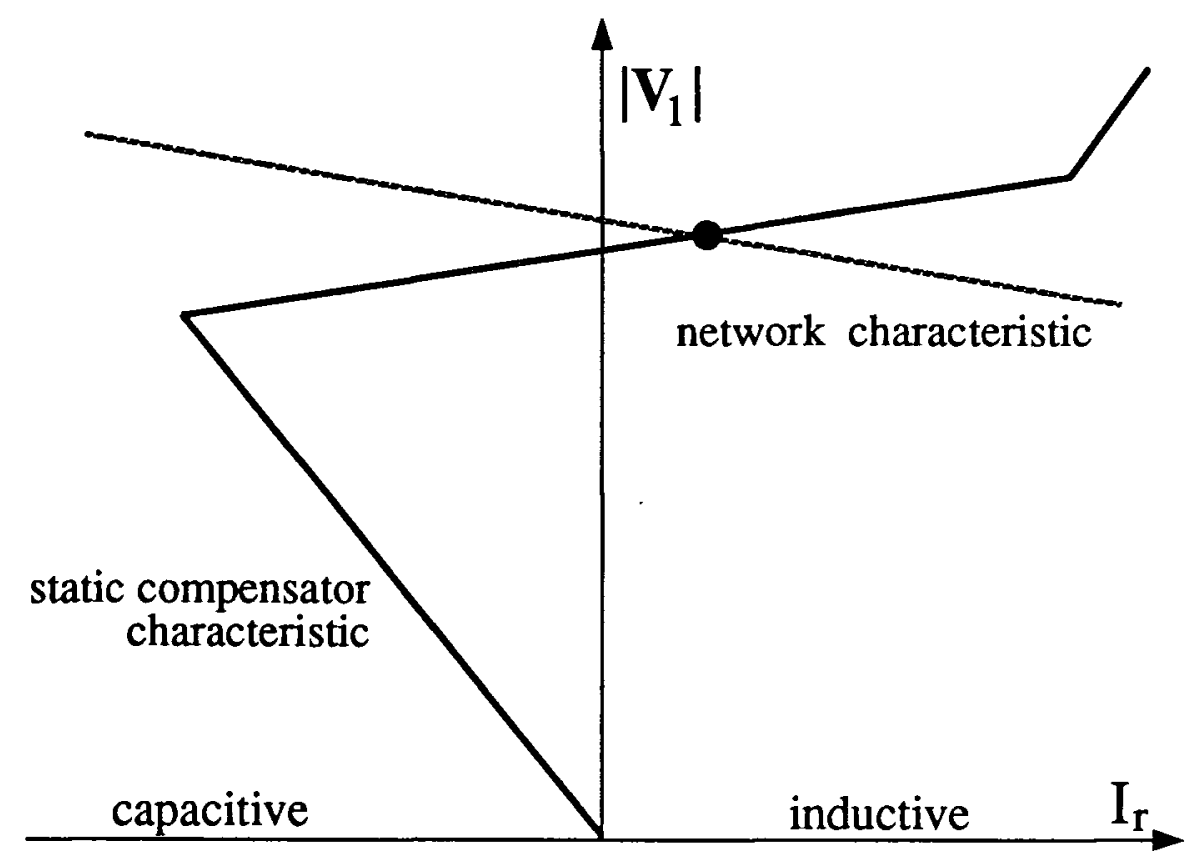

Figure 2.3: V-I characteristic of the static compensator.

current phasors are then used to compute the right-hand side

$$
f(\sigma)=V_{s}+I_{r} K
$$

of Eq. (2.13). By varying the conduction angles, the curve of Figure 2.4 for $f(\sigma)$ is obtained point by point. For a given voltage $\left|V_{1}\right|$, the correct $\sigma$ is then simply the intersection of $f(\sigma)$ with the horizontal line $\left|V_{1}\right|$. This intersection is found with the secant method [24].

Once the conduction angle has been found through this sub-iteration, the TCR can again be represented by the current sources of Eq. (2.5), which are then used for the harmonic iteration described earlier. To start the overall iterative process, the load flow at fundamental frequency is first solved. The compensator busses are treated as PV busses with $\mathrm{P}=0$ and $\left|\mathrm{V}_{1}\right|=V_{\text {s }}$ in this initial load flow solution. 


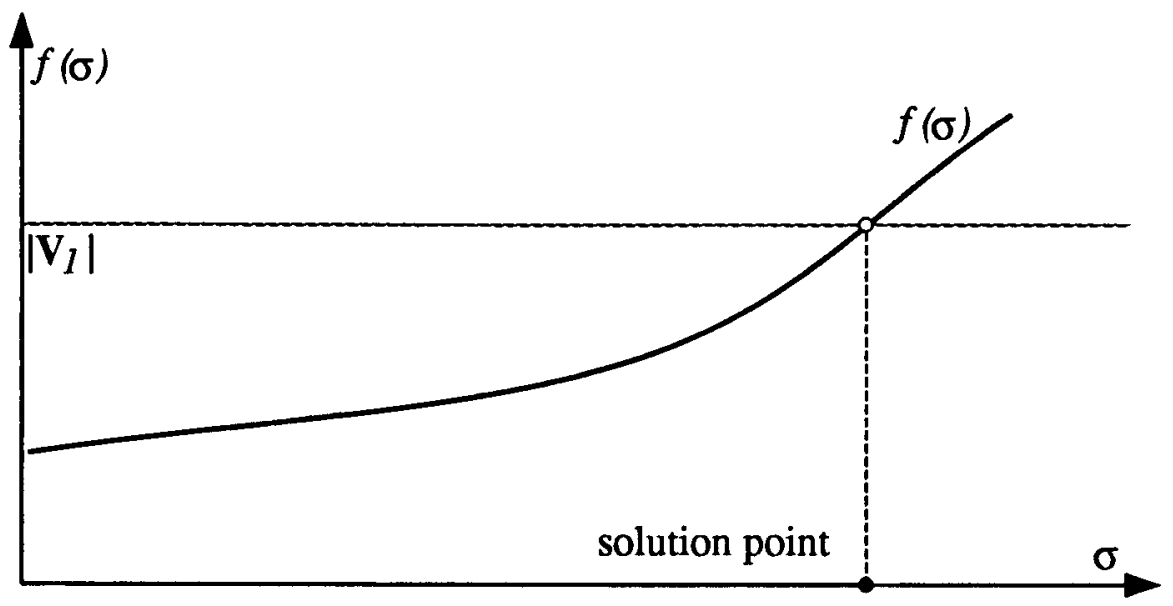

Figure 2.4: Solution for conduction angle.

\subsection{Discussion of Convergence Behaviour}

The method of including control characteristics of static compensator seemed simple at first, but failed to converge when first implemented for the following reason: With a given bus voltage $\left|\mathbf{V}_{1 a}\right|$, the iteration for conduction angle provides a current on the compensator characteristic at point a of Figure 2.5. Injecting this current into the network and solving for the fundamental frequency bus voltages leads to point $b$. The voltage in point $b$ is then used to calculate a new conduction angle and current injection (point $c$ ). This is the process on which the harmonic iteration, as originally conceived, is based. As shown in Figure 2.5, the iteration diverges because the slopes of the compensator and network characteristics do not meet the criteria required for convergence.

Convergence may be achieved by iterating in the reverse direction (as seen in the figure from a to I and to II). This approach is not always reliable, however, because the network line may slope in such a way that the convergence criteria for the reverse iteration are violated. Instead of reverse iteration, a reliable method was proposed to overcome the convergence difficulty and also to preserve the advantages of the original 


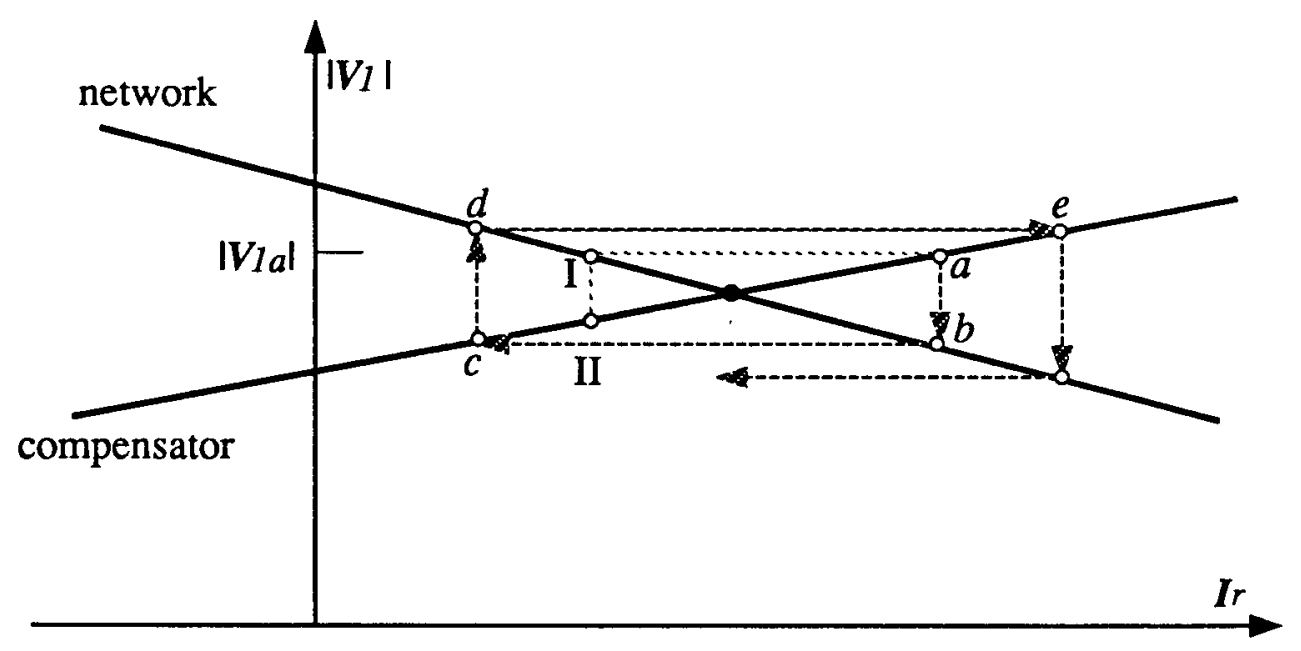

Figure 2.5: Divergence of the iterative process.

harmonic iteration method. The basic idea of this method is to compute a new point c' closer to the solution point by using a sloped line $\mathrm{S}$, instead of a horizontal line, as shown in Figure 2.6. Ideally, the slope of this line $\mathrm{S}$ should be close to the slope of the network characteristic. If the short-circuit impedance of rest of the network viewed from the compensator location is known, then its value is the best possible slope. If it is not known, then it can be estimated from an approximate network characteristic

$$
\left|\mathbf{V}_{1}\right|=E-I_{\tau} X_{s}
$$

where $E$ and $X_{s}$ are values obtained from successive iterations (e.g., points b' and d' in Figure 2.6). The modified network characteristic (line S) is then solved with the compensator characteristic, to obtain a new bus voltage $\left|\mathbf{V}_{1}\right|$. Since the actual network characteristic is almost a straight line as long as one is far away from voltage instability, the linear approximation of Eq. (2.15) becomes quite accurate after two iterations. Consequently, the convergence behaviour of this approach is very good. 


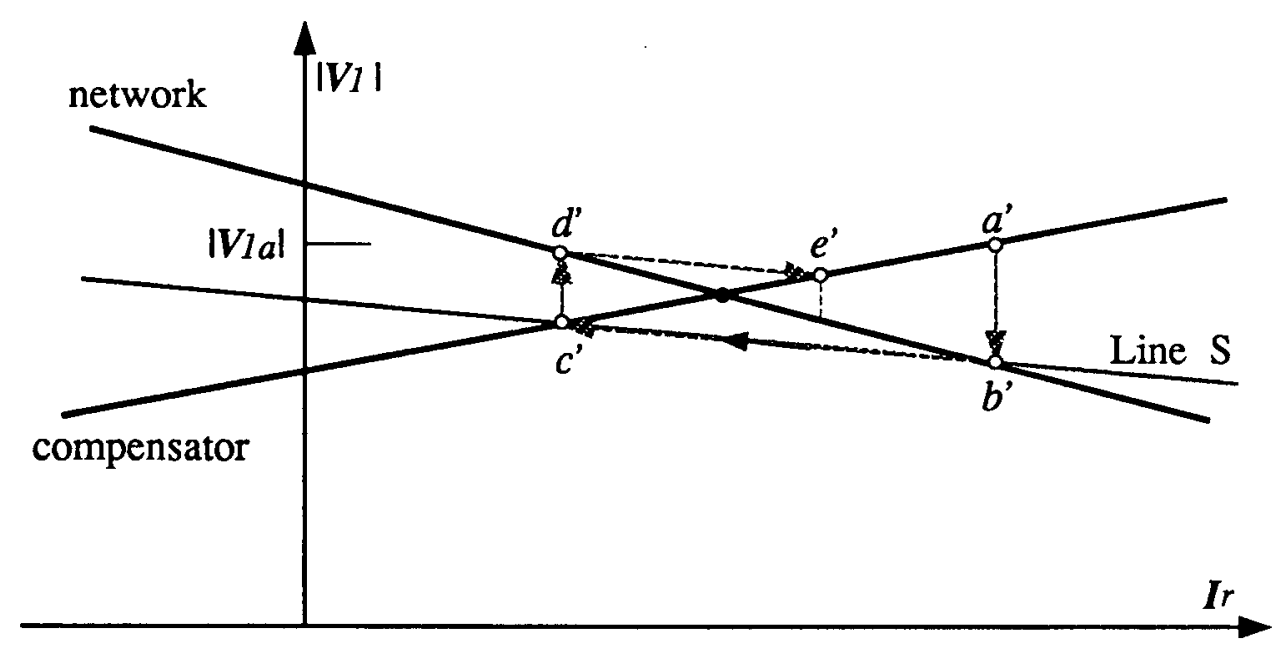

Figure 2.6: Improved iterative process.

The overall iterative technique can now be explained with Eqs. (2.10), (2.11) and (2.12). First of all, there are two sub-iterations implemented in the solution process. One is the sub-iteration to include the static compensator control characteristic inside Eq. (2.10). This is a single-variable problem without any convergence problems. The second sub-iteration is the solution of Eq. (2.11), which is basically the ordinary load flow problem with extra current injections for compensators. Considerable experience has been accumulated for this problem, and its solution with the Newton-Raphson method is quite reliable now.

The proposed iterative technique is a multivariable fixed point iterative process [24]. Convergence of the iterations between Eq. (2.10) and Eq. (2.11) is generally fast, as explained in Figure 2.6. For the iterations between Eq. (2.12) and the rest of the equations, no convergence difficulties have been found for normal cases so far. This may partly be due to the low pass filter effects of the TCR. Because of these effects, the harmonic 
voltage changes at the TCR terminal do not influence the harmonic current injections into the system significantly. The fact that the network harmonic characteristic is just a passive impedance and the TCR a harmonic current source with a large impedance in parallel makes convergence easier as well. The iterative process for one particular harmonic is illustrated in Figure 2.7.
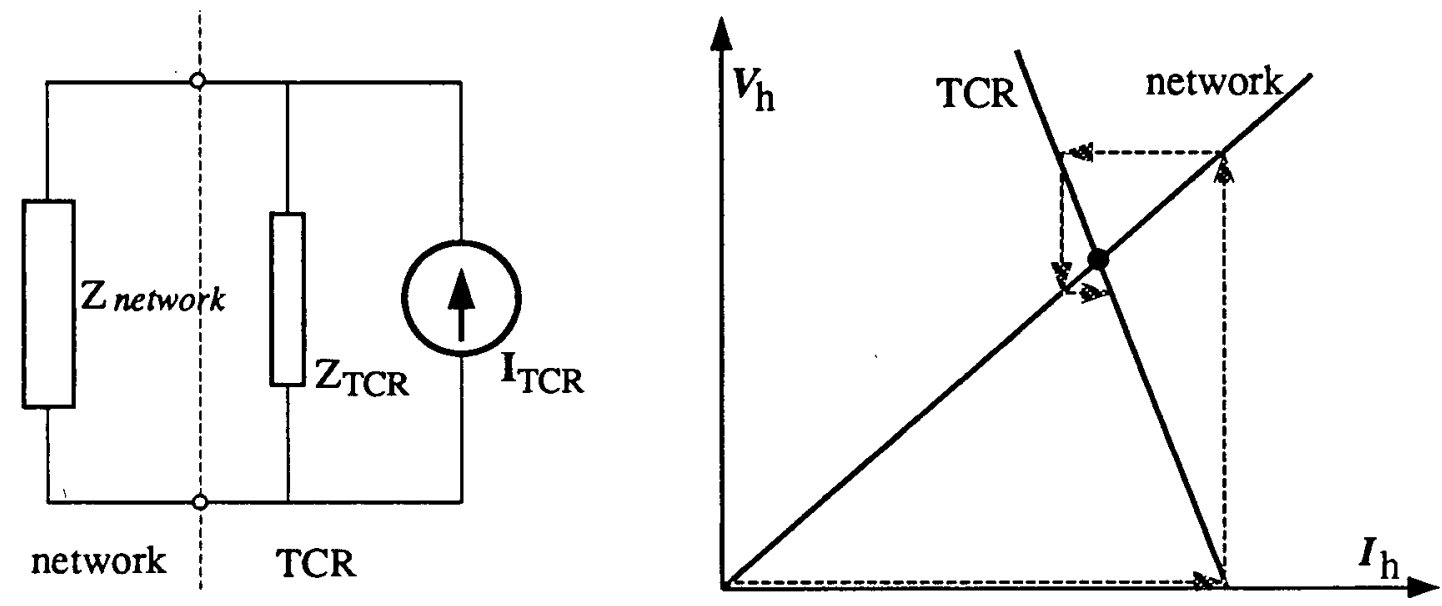

Figure 2.7: Iteration of harmonic component.

\subsection{Ilustrative Examples}

To illustrate the computation of steady-state harmonics in networks with static compensators, three test systems are studied:

1. A practical 19 bus system of TransAlta Utilities Corporation with one static compensator [25] (data are listed in Appendix E). The model representation of that compensator is as shown in Figure 2.2. 
2. A 9 bus system which is an equivalent of the above system. The 11th, 13th and 23rd harmonic filters were removed in this case.

3. A modified IEEE 14 bus test system [26]. Two fictitious static compensators are connected to busses 6 and 8 , respectively. Each of them is assumed to have one twowinding transformer with one TCR set. There are no filters at all. The transformer connection at bus 6 is $\mathrm{Y}-\mathrm{Y}$, and that at bus 8 is $\mathrm{Y}-\Delta$.

The computations were done in per-unit values, with a base power of 100 MVA. Analysis up to the 23rd harmonic is performed.

\subsubsection{Comparison with Field Measurements}

Field measurements of the first system were made available to this work [25]. These are then used to compare against simulated results. The case with the capacitor banks disconnected at a conduction angle of $39.8^{\circ}$ was chosen. Converged solutions were obtained without difficulty. Both measured and computed waveforms are plotted in Figure 2.8. As shown in the figures, all simulated results correlate well with the measured ones, if one considers the fact that the system data and the load flow conditions are only an approximation for the real operating conditions.

\subsubsection{Harmonic Load Flow Solutions}

The program is then used to compute harmonic load flow distributions of all test systems. Table 2.1 shows the voltage solutions of the three systems with fundamental frequency and with the harmonics of order $5,7,11$ and 13 at two busses. The last column provides the percentage of harmonic distortion (\%HD) [1] of the bus voltages.

Note that the 5 th and 7 th harmonic voltages are zero at the compensator busses for the first two systems. This phenomena is as expected due to the fact that the 5 th and 

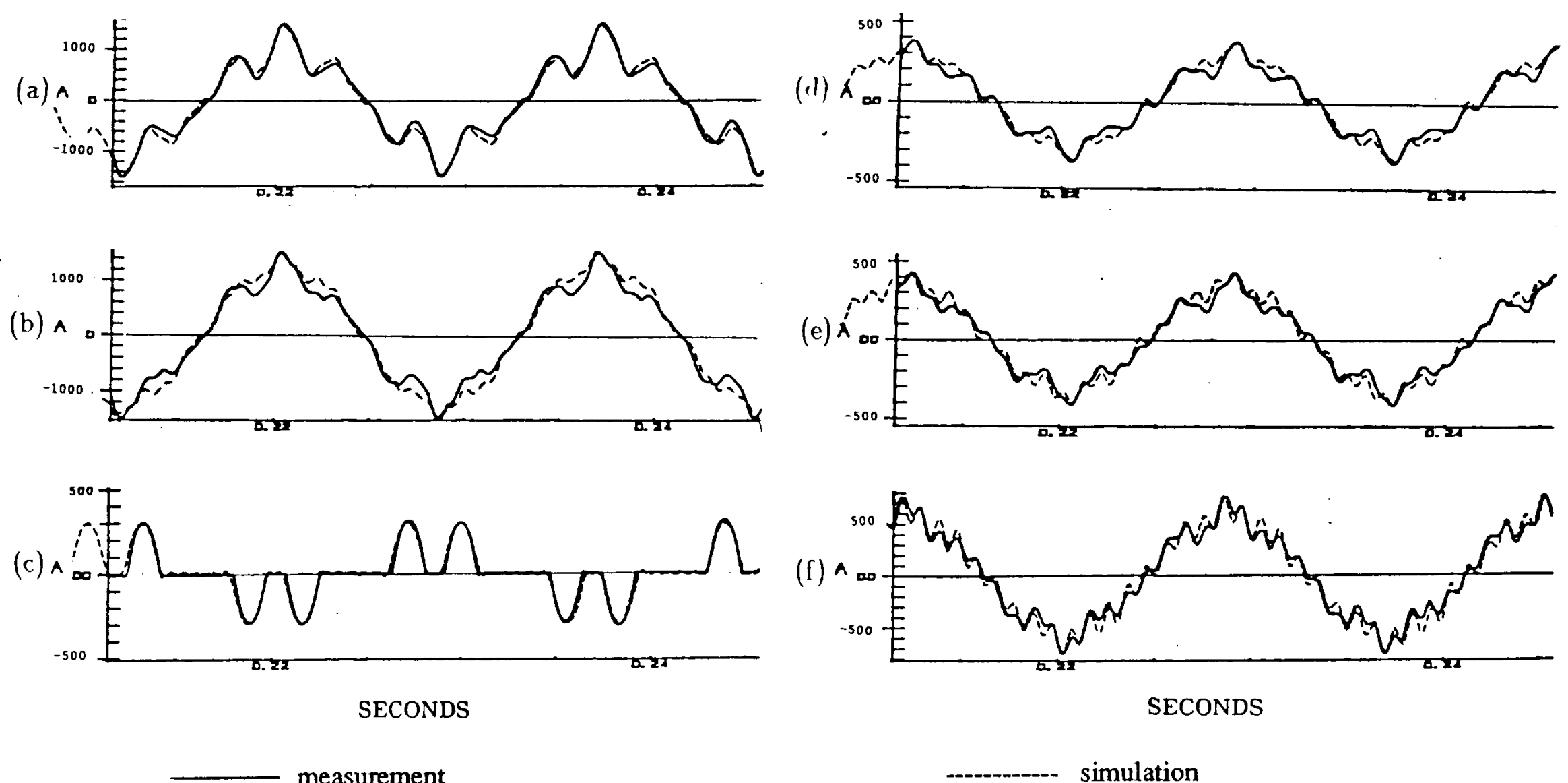
(a) $\mathrm{Y}$ - side $\mathrm{C}$ - phase current
(b) $\mathrm{Y}$ - side $\mathrm{C}$ - phase total filter current
(c) $Y$ - side $C$ - phase TCR current

(d) $\mathrm{Y}$ - side $\mathrm{C}$-phase 11 th filter current

(e) $\mathrm{Y}$ - side $\mathrm{C}$ - phase 13th filter current

(f) $\mathrm{Y}$ - side $\mathrm{C}$ - phase 23 rd filter current

Figure 2.8: Waveform comparison with field measurements. 
7th harmonics are cancelled out in the three-winding connection of the transformer of Figure 2.2. These harmonics are observed at the TCR terminal, however, though their magnitudes are small. The harmonic reduction is due to the presence of delta-connected capacitor bank which acts as a 5th harmonic filter. All of these observations agree with the reported field measurements (harmonic distortion less than $1 \%$ at the compensator bus). For the test system 3 , significant harmonic distortion occurs, due to the absence of harmonic filters and three-winding transformer connections.

Table 2.1: Bus voltage harmonics.

\begin{tabular}{|c|c|rrrrr|c|}
\hline System & Bus & 1st & 5th & 7th & 11th & 13th & \%HD \\
\hline \hline 1 & COM & 1.0221 & 0.0000 & 0.0000 & 0.0002 & 0.0001 & 0.02 \\
& & $/-27.2$ & $/-158.2$ & $/ 151.9$ & $/ 42.9$ & $/ 20.4$ & \\
& TCR & 0.9926 & 0.0061 & 0.0044 & 0.0005 & 0.0011 & 0.97 \\
& (Y-side) & $/-27.2$ & $/ 20.9$ & $/-19.9$ & $/ 62.4$ & $/ 88.2$ & \\
\hline \hline 2 & COM & 1.0150 & 0.0000 & 0.0000 & 0.0032 & 0.0015 & 0.36 \\
& & $/-9.4$ & $/-89.2$ & $/-78.1$ & $/ 46.1$ & $/-73.5$ & \\
& TCR & 0.9542 & 0.0056 & 0.0034 & 0.0098 & 0.0087 & 1.58 \\
& (Y-side) & $/ 80.7$ & $/-152.4$ & $/-158.5$ & $/-18.8$ & $/-38.3$ & \\
\hline \hline $3-(1)$ & COM & 1.0212 & 0.0373 & 0.0127 & 0.0028 & 0.0012 & 3.87 \\
& & $/-13.0$ & $/ 117.8$ & $/ 85.6$ & $/ 32.4$ & $/-173.6$ & \\
& TCR & 1.0120 & 0.0595 & 0.0186 & 0.0044 & 0.0018 & 6.18 \\
& & $/-13.0$ & $/ 120.4$ & $/ 90.6$ & $/ 36.4$ & $/-167.7$ & \\
\cline { 2 - 7 } $3-(2)$ & COM & 1.0481 & 0.0562 & 0.0041 & 0.0024 & 0.0010 & 5.38 \\
& & $/-12.9$ & $/-64.5$ & $/ 88.1$ & $/-141.4$ & $/-170.4$ & \\
& TCR & 1.0575 & 0.0728 & 0.0040 & 0.0031 & 0.0011 & 6.90 \\
& & $/-42.9$ & $/-34.3$ & $/ 63.9$ & $/-112.4$ & $/ 162.4$ & \\
\hline
\end{tabular}

1 - Phase angle in degrees.

COM - The bus to which the compensator is connected.

TCR - The bus to which the TCR set is connected.

$3-(1)$ - Compensator 1 of test system 3 .

The harmonic current injections into the system at the compensator busses are listed in Table 2.2. Also listed in the tabie are the computed TCR conduction angles in degrees. 
The current distortion is larger than that of the voltages. This trend is also reported in the field measurements [25]. Note the 5th and 7th harmonic currents are nonzero, though of small amplitude. It is caused by slight differences between the impedances of $\mathrm{Y}$ and $\Delta$ secondary windings of the transformer. The current distortion is even larger in system 3 . This is again due to the absence of filters and three-winding transformers, and also due to the small conduction angles.

Table 2.2: Static compensator current injections and conduction angles.

\begin{tabular}{|l|c|ccccc|r|}
\hline System & $\sigma$ & 1st & 5th & 7th & 11th & 13th & \%HD \\
\hline \hline 1 & $134.7^{\circ}$ & 0.4847 & 0.0003 & 0.0001 & 0.0005 & 0.0014 & 0.31 \\
2 & $135.9^{\circ}$ & 1.0009 & 0.0002 & 0.0001 & 0.0101 & 0.0119 & 1.58 \\
$3-(1)$ & $91.8^{\circ}$ & 0.1214 & 0.0582 & 0.0113 & 0.0020 & 0.0006 & 48.86 \\
$3-(2)$ & $72.2^{\circ}$ & 0.1235 & 0.0442 & 0.0008 & 0.0009 & 0.0002 & 35.80 \\
\hline
\end{tabular}

Values shown in the table are the current magnitude.

3 -(1) indicates compensator 1 of test system 3 .

\subsubsection{Convergence Behaviour}

The convergence behaviour of the proposed technique was excellent. In all cases tested, less than six iterations were needed to obtain converged solutions with an accuracy of $10^{-4}$ per-unit. A detailed convergence behaviour is listed in Table 2.3 for the three test systems, along with the computer CPU times. ${ }^{1}$

The change of the conduction angles during the iterations is shown in Table 2.4. Both tables indicate fast convergence characteristics of the method. For all the three test cases, no difficulties have been encountered from the initial guess of zero harmonic bus voltages. Because the magnitudes of the initial harmonic voltages are zero, no estimates are needed for their phase angles.

\footnotetext{
${ }^{1}$ All computer times reported in this dissertation were recorded on the VAX-11/750 computer of Electrical Enginering Department, University of British Columbia.
} 
Table 2.3: Bus voltage convergence and solution times.

\begin{tabular}{|c||cc|cc|cc|}
\hline \multicolumn{1}{|c||}{ Iteration } & \multicolumn{2}{c|}{ system-1 } & \multicolumn{2}{c|}{ system-2 } & \multicolumn{2}{c|}{ system-3 } \\
\cline { 2 - 7 }$\#$ & $\Delta V_{1}$ & $\Delta V_{H}$ & $\Delta V_{1}$ & $\Delta V_{H}$ & $\Delta V_{1}$ & $\Delta V_{H}$ \\
\hline \hline 1 & 0.07558 & 0.00504 & 0.05000 & 0.00751 & 0.05000 & 0.05156 \\
2 & 0.03231 & 0.00307 & 0.03505 & 0.01064 & 0.00121 & 0.00849 \\
3 & 0.01897 & 0.00039 & 0.01660 & 0.00096 & 0.00047 & 0.00246 \\
4 & 0.00018 & 0.00004 & 0.00006 & 0.00008 & 0.00032 & 0.00055 \\
5 & 0.00006 & 0.00000 & & & 0.00016 & 0.00022 \\
6 & & & & & 0.00010 & 0.00004 \\
\hline \hline CPU(s) & \multicolumn{2}{|c|}{34.8} & \multicolumn{2}{|c|}{34.9} \\
\hline
\end{tabular}

$\Delta V_{1}$ - The maximum update of the fundamental frequency voltages.

$\Delta V_{H}$ - The maximum update of the harmonic voltages.

Table 2.4: Convergence of conduction angles (in degrees).

\begin{tabular}{|c||c|c|cc|}
\hline Iteration & system-1 & system-2 & \multicolumn{2}{|c|}{ system-3 } \\
\cline { 4 - 5 }$\#$ & & & $\sigma_{1}$ & $\sigma_{2}$ \\
\hline \hline 0 & 114.59 & 114.59 & 114.59 & 114.59 \\
1 & 129.46 & 123.43 & 93.28 & 75.20 \\
2 & 134.67 & 135.70 & 92.05 & 72.70 \\
3 & 134.72 & 135.99 & 91.86 & 72.35 \\
4 & 134.73 & 135.97 & 91.81 & 72.28 \\
5 & 134.73 & & 91.80 & 72.27 \\
6 & & & 91.80 & 72.27 \\
\hline
\end{tabular}

\subsection{Conclusions}

Based on the principle of harmonic iteration, a single-phase harmonic load flow method has been proposed as the basis of the multiphase harmonic load flow (MHLF) technique to be developed in this work. Its application for the harmonic analysis of static compensators was found to be successful, as indicated by both the field measurement comparisons and the results from a number of test cases. With respect to the ultimate goal of the MHLF development, this newly developed method offers many attractive features: 
1. The limitations of other harmonic iteration approaches $[13,14]$ on modelling the load flow constraints and the control characteristics of nonlinear elements have been removed.

2. The method exhibited good convergence behaviour. Initial guess of zero harmonic conditions is straightforward. On the other hand, it is difficult to find a proper starting point for the HARMFLO technique. This problem has considerably reduced the convergence rate of that technique [15].

3. This method is also efficient, due to the use of frequency-domain steady-state analysis of the linear network. Since the harmonics are decoupled in such a network, the computational requirement is minimized and confined to only a regular load flow solution and a harmonic frequency scan. On the other hand, the HARMFLO technique needs the computation of the harmonic Jacobian matrix. This matrix is very large because of the coupling nature of all the harmonics.

4. The method is very versatile. It is easy to analyze other nonlinearities with or without control characteristics with this method.

In addition, the new method presents no major difficulties for multiphase extension, as long as the problems of multiphase fundamental frequency load flow are solved. This leads to the development of a new multiphase load flow solution technique, as shown in the next chapter. 


\section{Chapter 3}

\section{Multiphase Load Flow Solutions}

Based on the encouraging results of the last chapter, the proposed single-phase harmonic load flow method has been selected for multiphase expansion. Two major conclusions can be drawn from the single-phase method:

1. A load flow technique with frequency scan capability is needed for the steady-state solution of the linear network.

2. A frequency-domain model is needed to represent the nonlinear element, with or without control specifications.

The above conclusions are also applicable to the multiphase harmonic load flow technique. For this case, however, all problems must be considered in the multiphase framework. This chapter deals with the first problem, the development of a multiphase (fundamental frequency) load flow solution method.

\subsection{Introduction}

A multiphase load flow solution is not only essential for the harmonic analysis which is the primary concern of this work, the technique is also useful by itself to investigate the unbalanced operations of power systems at the power frequency. Although considerable progress has been made on the subject of unbalanced load flow analysis $[17,18]$, there are still many problems to be solved. 
The first problem is the load modelling in the multiphase environment. A load has traditionally been modelled as an individual phase-to-ground real and reactive power consumption. Apparently, this model, which is derived directly from the positive sequence load flow case, fails to include the mutual couplings among phases of the load. In a real situation, it may lead to incorrect conclusions on, for instance, the distribution of the negative sequence currents. As a matter of fact, some field measurements have revealed that such errors are severe [27].

The way of formulating load flow equations presents another problem with the existing techniques. All these formulations follow the customary approach of node-to-ground power constraints. While the node-to-ground power consumption is exclusively true in the positive sequence load flow studies, this single-phase concept can hardly be useful in the multiphase environment, as there are many cases where the load flow constraints are defined in the forms of phase-to-phase or node-to-node. Such examples would include the loads connected between two phases and delta connected generators. There is therefore a strong need for better approaches which can handle other possible system configurations.

The complexities resulted from the multiphase analysis also create a problem of finding a starting point for the numerical solution processes, as all load flow solution methods are iterative. For the positive sequence load flow solutions, a straightforward 'flat' start [21] is generally satisfactory for most solution methods. In the multiphase case, however, the accumulated phase shifts across transformer connections and other 'irregular' connections can often lead to a large change in the phase angles of the node voltages. This makes the selection of a starting point very difficult. Although it is well-known that a proper starting point is critical to the convergence of some numerical methods, no work has been found to address the multiphase starting point problem.

In view of this situation, none of the existing methods are suitable for comprehensive 
multiphase load flow analysis. A systematic study of the problem is therefore necessary. This leads to the development of a new multiphase load flow technique.

\subsection{Modelling of Multiphase Network Components}

As explained before, a new formulation is needed to setup the multiphase load flow equations. In contrast to most existing methods, the formulation proposed in this work is based on modelling both the power system components and their load flow constraints in branch level, instead of constraints on node quantities. Since branches can be connected in any way by the user, this provides greater flexibility.

\subsubsection{Three-phase Synchronous Machines}

The response of a synchronous machine is different for the positive, negative, or zero sequence current injections. This must be taken into account in unbalanced load flow studies. Reference [17] has studied this problem and suggested that three equations are required to describe the behaviour of synchronous machines in the symmetrical components frame:

$$
\begin{aligned}
& \mathbf{I}_{k m-p}=\left(R_{\text {armature }}+j X_{q}\right)^{-1}\left(\mathbf{V}_{k-p}-\mathbf{V}_{m-p}-\mathbf{E}_{q}\right) \\
& \mathbf{I}_{k m-n}=Y_{n}\left(\mathbf{V}_{k-n}-\mathbf{V}_{m-n}\right) \\
& \mathbf{I}_{k m-0}=Y_{o}\left(\mathbf{V}_{k-0}-\mathbf{V}_{m-0}\right) .
\end{aligned}
$$

where,

Subscripts $\mathrm{k}$ and $\mathrm{m}$ refer to the machine terminals;

$\mathrm{V}_{k}$ and $\mathrm{V}_{m}$ are the node voltages;

$\mathrm{I}_{k m}$ represents the currents flowing from side $\mathrm{k}$ to side $\mathrm{m}$;

$\mathbf{E}_{q}$ is an equivalent excitation voltage. 
Subscripts $\mathrm{p}, \mathrm{n}$, and $\mathrm{o}$ indicate the positive, negative, and zero sequence components, respectively. For the negative sequence reactance, $j \omega\left(L_{d}^{\prime \prime}+L_{q}^{\prime \prime}\right) / 2$ can be used. The zero sequence reactance is $j \omega L_{o}$. For the resistance, the armature resistance can be used, though this is not quite correct because the negative sequence resistance can be an order of magnitude larger ${ }^{1}$.

The phase domain representation of the machine can be obtained by applying the inverse symmetrical components transformation to Eqs. (3.1)-(3.3). However, since the negative and positive sequence admittances are not equal, it leads to an unsymmetrical machine admittance matrix. This problem can be solved by defining a new internal voltage $\mathrm{E}_{p}$ as follows:

$$
\mathbf{E}_{p}=\mathbf{V}_{k-p}-\mathbf{V}_{m-p}-Y_{n}^{-1}\left(R_{\text {armature }}+j X_{q}\right)^{-1}\left(\mathbf{V}_{k-p}-\mathbf{V}_{m-p}-\mathbf{E}_{q}\right)
$$

Equation (3.1) is then modified into

$$
\mathbf{I}_{k m-p}=Y_{n}\left(\mathbf{V}_{k-p}-\mathbf{V}_{m-p}-\mathbf{E}_{p}\right)
$$

Transferring Eqs. (3.2), (3.3) and (3.4) into the phase domain, and using brackets to denote matrices, we have

$$
\left[\mathbf{I}_{k m}\right]=\left[Y_{g}\right]\left(\left[\mathbf{V}_{k}\right]-\left[\mathbf{V}_{\mathbf{m}}\right]-[\mathbf{E}]\right)
$$

where

$$
\begin{aligned}
& {\left[\begin{array}{ll}
\mathbf{I}_{k m}
\end{array}\right]=\left[\begin{array}{lll}
\mathbf{I}_{k m-a} & \mathbf{I}_{k m-b} & \mathbf{I}_{k m-c}
\end{array}\right]^{T}} \\
& {\left[\begin{array}{lll}
\mathbf{V}_{k}
\end{array}\right]=\left[\begin{array}{lll}
\mathbf{V}_{k-a} & \mathbf{V}_{k-b} & \mathbf{V}_{k-c}
\end{array}\right]^{T}} \\
& {\left[\begin{array}{lll}
\mathbf{V}_{m}
\end{array}\right]=\left[\begin{array}{lll}
\mathbf{V}_{m-a} & \mathbf{V}_{m-b} & \mathbf{V}_{m-c}
\end{array}\right]^{T}} \\
& {[\mathbf{E}]=\left[\begin{array}{lll}
\mathbf{E}_{p} & a^{2} \mathbf{E}_{p} & a \mathbf{E}_{p}
\end{array}\right]^{T}} \\
& a=e^{-j 2 \pi / 3} .
\end{aligned}
$$

\footnotetext{
${ }^{1}$ More accurate modelling of synchronous machines is described in Chapter 5.
} 
Matrix $\left[Y_{g}\right]$ is the machine's admittance matrix in phase quantities. This is a symmetric matrix with mutual quantities equal to $\left(Y_{o}-Y_{n}\right) / 3$, and self quantities equal to $\left(Y_{o}+\right.$ $\left.2 Y_{n}\right) / 3$. The machine internal voltage $\mathbf{E}_{p}$ is unknown and must be adjusted to satisfy the machine load flow constraints for terminal voltage and power output, as follows:

(a) Slack machine. The specified constraints are the magnitude and the phase angle of the positive sequence voltage at the machine terminals.

$$
[T]\left(\left[\mathbf{V}_{k}\right]-\left[\mathbf{V}_{m}\right]\right)=\mathbf{V}_{\text {specified }}
$$

where

$$
[T]=(1 / 3)\left[\begin{array}{lll}
1 & a & a^{2}
\end{array}\right]
$$

Machines with these constraints correspond to the slack nodes in conventional load flow studies. Under unbalanced conditions, the negative and zero sequence voltages will be nonzero because the network sees the machine as admittances $Y_{n}$ and $Y_{o}$ in negative and zero sequence representations.

(b) PV machine. The specified constraints are the three-phase active power output and the magnitude of the positive sequence voltage at the machine terminals.

$$
\begin{gathered}
\operatorname{Real}\left\{-\left[\mathbf{I}_{k m}\right]^{H}\left(\left[\mathbf{V}_{k}\right]-\left[\mathbf{V}_{m}\right]\right)\right\}=P_{\text {epecified }} \\
\left|[T]\left(\left[\mathbf{V}_{k}\right]-\left[\mathbf{V}_{m}\right]\right)\right|=V_{\text {specified }},
\end{gathered}
$$

where superscript ${ }^{H}$ denotes conjugate transposed.

(c) PQ machine. The specified constraints are the three-phase active and the threephase reactive power output.

$$
-\left[\mathbf{I}_{k m}\right]^{H}\left(\left[\mathbf{V}_{k}\right]-\left[\mathbf{V}_{m}\right]\right)=(P+j Q)_{\text {specified }}
$$


The constraints for either type of machine can be generalized as

$$
G\left(\left[\mathbf{I}_{k m}\right],\left[\mathbf{V}_{k}\right],\left[\mathbf{V}_{m}\right]\right)=F_{\text {specified }} .
$$

This equation and Eq. (3.5) jointly define the three-phase machine model in a branch form, as shown in Figure 3.1(a).

(a)

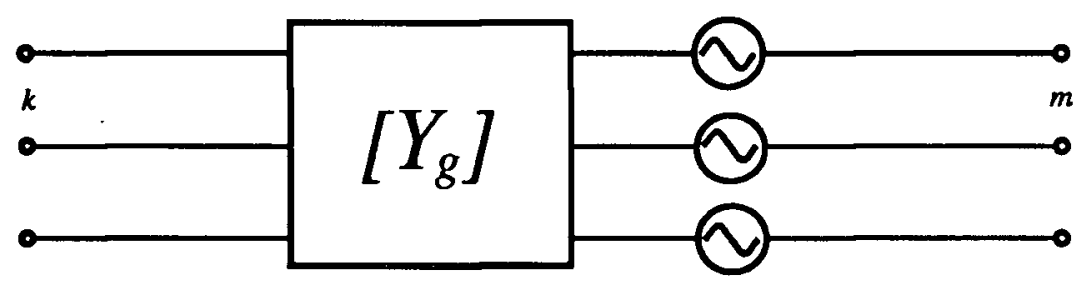

(b)

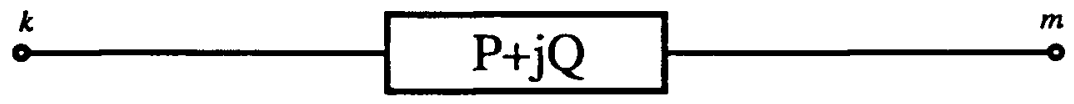

(c)

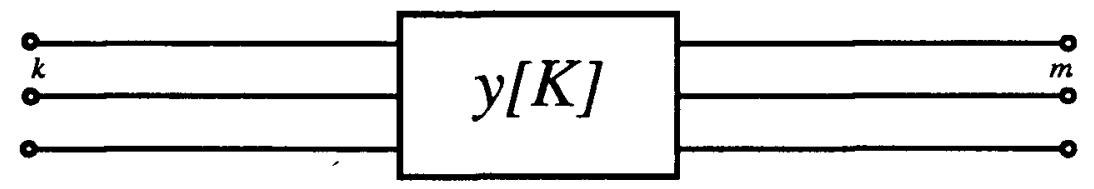

(d)

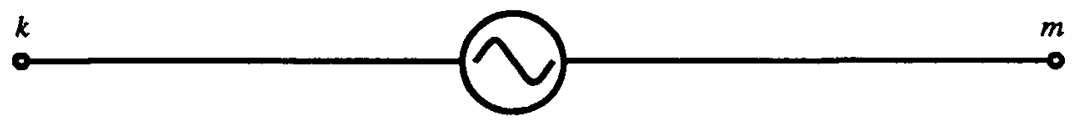

(e)

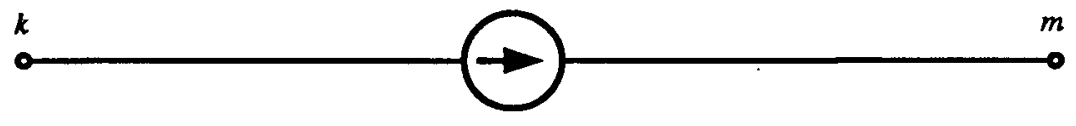

(a) three-phase synchronous machine.

(b) individual branch load. (c) three-phase static load.

$\begin{array}{ll}\text { (d) voltage source. } & \text { (e) current source. }\end{array}$

Figure 3.1: Branch models for multiphase load flow analysis. 


\subsubsection{Multiphase Loads}

Loads are usually represented as constant power consumption in single-phase (positive sequence) load flow programs. For unbalanced load flow analysis, in addition to the power constraints, the different load responses to positive, negative and zero sequence voltages and currents must also be considered. In view of the variety of load characteristics, four basic types of load models are proposed.

Type 1. Constant impedance load with known $[\mathrm{Z}]$ or $[\mathrm{Y}]$ branch-matrix representation.

Type 2. Load with constant active and reactive power specified as a single-phase branch (Figure $3.1(\mathrm{~b})$ ). It is defined as:

$$
\mathbf{I}_{k m}^{H}\left(\mathbf{V}_{k}-\mathbf{V}_{m}\right)=(P+j Q)_{\text {specified }}
$$

This load corresponds to the traditional PQ representation. However, it is defined as a branch between two nodes, rather than from node to ground as in single-phase load flow programs. This allows large flexibility in the type of connection, as for example phase-to-phase loads in delta systems.

Type 3. Static load (Figure 3.1(c)). In this type of load, it is assumed that the positive and negative sequence impedances are equal and that the ratio of the positive to zero sequence impedance is available. In effect, it is assumed that the load impedances are balanced for the three phases. The total active and reactive power is specified, but the positive and zero sequence impedances are not explicitly known. Using symmetrical components, the branch equations for this type of load in phase quantities can be expressed as

$$
\begin{gathered}
{\left[\mathbf{I}_{k m}\right]=y[K]\left(\left[\mathbf{V}_{k}\right]-\left[\mathbf{V}_{m}\right]\right)} \\
{\left[\mathbf{I}_{k m}\right]^{H}\left(\left[\mathbf{V}_{k}\right]-\left[\mathbf{V}_{m}\right]\right)=(P+j Q)_{\text {specified }}}
\end{gathered}
$$


where $y$ is an unknown admittance and $[K]$ is a known constant symmetric matrix determined from the positive to zero sequence impedance ratio. The unknown admittance must be adjusted to fulfill Eq. (3.13).

Type 4. Rotating machine load with unequal negative and positive sequence impedances, as in the case of induction motors. For this load, it is assumed that both the negative and zero sequence admittances are known. The positive sequence admittance is not known and is to be determined from the three-phase active and reactive power consumption. With symmetrical components, this type of load can be represented as

$$
\begin{aligned}
& \mathbf{I}_{k m-p}=Y_{p}\left(\mathbf{V}_{k-p}-V_{m-p}\right) \\
& I_{k m-n}=Y_{n}\left(V_{k-n}-V_{m-n}\right) \\
& I_{k m-o}=Y_{o}\left(V_{k-o}-V_{m-o}\right)
\end{aligned}
$$

In stead of using $Y_{p}$ as an unknown, it is better to model the load as an internal voltage $\mathbf{E}_{p}$ behind the negative sequence admittance $Y_{n} . \mathbf{E}_{p}$ then becomes the unknown variable and Eq. (3.14) can be rewritten as

$$
\mathbf{I}_{k m-p}=Y_{n}\left(\mathbf{V}_{k-p}-\mathbf{V}_{m-p}-\mathbf{E}_{p}\right)
$$

Transforming Eqs. (3.15), (3.16) and (3.17) into the phase domain and including the built-in load flow constraints, this load model can be defined as:

$$
\begin{gathered}
{\left[\mathbf{I}_{k m}\right]=\left[Y_{g}\right]\left(\left[\mathbf{V}_{k}\right]-\left[\mathbf{V}_{m}\right]-[\mathbf{E}]\right)} \\
-\left[\mathbf{I}_{k m}\right]^{H}\left(\left[\mathbf{V}_{k}\right]-\left[\mathbf{V}_{m}\right]\right)=(P+j Q)_{\text {specified }}
\end{gathered}
$$

where

$$
[\mathbf{E}]=\left[\begin{array}{lll}
\mathbf{E}_{p} & a^{2} \mathbf{E}_{p} & a \mathbf{E}_{p}
\end{array}\right]^{T}
$$




$$
\begin{gathered}
{\left[Y_{g}\right]_{\text {mutual }}=\left(Y_{o}-Y_{n}\right) / 3} \\
{\left[Y_{g}\right]_{\text {oelf }}=\left(Y_{o}+2 Y_{n}\right) / 3}
\end{gathered}
$$

Note that with the introduction of $\mathbf{E}_{p}$, this type of load has the same structure as the $\mathrm{PQ}$ synchronous machine. It can then be simply treated as a $\mathrm{PQ}$ machine with negative power generation.

The assumption of constant power consumption may not be quite accurate for some loads with strong voltage dependence. Options have been provided in the technique to include the following load-voltage characteristics [30]:

$$
\begin{aligned}
& P=P_{\text {specified }}\left(\left|\mathbf{V}_{p}\right| / V_{r}\right)^{\alpha} \\
& Q=Q_{\text {specified }}\left(\left|\mathbf{V}_{p}\right| / V_{r}\right)^{\beta}
\end{aligned}
$$

where $\alpha$ and $\beta$ are the known load characteristic parameters, $\mathrm{V}_{p}$ is the positive sequence voltage seen by the load, and $V_{r}$ is the rated voltage of the load.

By specifying the load flow constraints at the branch level, the capability of load modelling is no longer limited to each bus with one of the load models. Loads can be arbitrarily connected between nodes or from node to ground. The combination of these basic types connected to the same bus is also allowed, thus providing the potential to model a wide variety of loading conditions.

\subsubsection{Voltage and current sources}

Voltage and current sources are again represented as branches. The voltage source (Figure $3.1(\mathrm{~d}))$ is defined as

$$
\mathrm{V}_{k}-\mathrm{V}_{m}=\mathrm{E}_{\text {specified }}
$$


A current source between two nodes (Figure 3.1(e)) is defined as currents leaving two nodes,

$$
\begin{aligned}
& \mathbf{I}_{m}=\mathbf{I}_{\text {specified }}, \\
& \mathbf{I}_{k}=-\mathbf{I}_{\text {specified }} .
\end{aligned}
$$

\subsubsection{Other Network Components}

Overhead transmission lines, underground cables, transformers, reactors and capacitors can all be modelled as coupled $\pi$ circuits. Details are well-documented in references [18] and [12]. There are no load flow constraints associated with these components.

\subsection{Multiphase Load Flow Equations and Its Solutions}

The load flow equations are formed by imposing power and voltage constraints on the network node quantities in the traditional approaches. As mentioned earlier, these approaches are very limiting in the multiphase environment. With the proposed branchmodelled load flow constraints for each component, there is no longer a need to consider the constraints for each node and hence arbitrary connections can easily be simulated.

\subsubsection{Formulation of Load Flow Equations}

The interaction of all the individual components is finally established through the network node current equations. With all components described at the branch level, it becomes easy to write the nodal equations for the entire network. The branch admittance matrix of each component without load flow constraints enters the larger network admittance matrix according to well-known building rules [12]. While the components with constraints (machines, voltage sources, and type-2, -3 and -4 loads) are modelled as unknown currents $\left[\mathbf{I}_{u}\right]$ leaving the nodes to which they are connected. As a result, the 
network equation is formed as

$$
[Y][\mathbf{V}]+\left[\mathbf{I}_{s}\right]+\left[\mathbf{I}_{\boldsymbol{u}}\right]=0
$$

where

$[Y]$ is the network node admittance matrix constructed from the components without load flow constraints, $[\mathbf{V}]$ is the node voltage vector,

$\left[\mathbf{I}_{\boldsymbol{B}}\right]$ is the vector of known current sources leaving each node, $\left[\mathbf{I}_{\mathbf{u}}\right]$ is the vector of unknown currents (associated with load flow constraints) leaving each node.

Collecting all the related equations together, the multiphase load flow problem can be formulated as:

$$
\begin{aligned}
\text { Network: } & {\left[f_{1}\right]=[Y][\mathbf{V}]+\left[\mathbf{I}_{s}\right]+\left[\mathbf{I}_{u}\right]=0 } \\
\text { Voltage source: } & f_{2}=\mathbf{V}_{k}-\mathbf{V}_{m}-\mathbf{E}_{\text {specified }}=0 \\
\text { Type-2 load: } & f_{3}=\mathbf{I}_{k m}^{H}\left(\mathbf{V}_{k}-\mathbf{V}_{m}\right)-(P+j Q)_{\text {specified }}=0 \\
\text { Machine constraints: } & f_{4}=G\left(\left[\mathbf{I}_{k m}\right],\left[\mathbf{V}_{k}\right],\left[\mathbf{V}_{m}\right]\right)-F_{\text {specified }}=0 \\
\text { Load-3 constraints: } & f_{5}=\left[\mathbf{I}_{k m}\right]^{H}\left(\left[\mathbf{V}_{k}\right]-\left[\mathbf{V}_{m}\right]\right)-(P+j Q)_{\text {specified }}=0 \\
\text { Machine: } & {\left[f_{6}\right]=\left[\mathbf{I}_{k m}\right]-\left[Y_{g}\right]\left(\left[\mathbf{V}_{k}\right]-\left[\mathbf{V}_{m}\right]-[\mathbf{E}]\right)=0 } \\
\text { Type-3 load: } & {\left[f_{7}\right]=\left[\mathbf{I}_{k m}\right]-y[C]\left(\left[\mathbf{V}_{k}\right]-\left[\mathbf{V}_{m}\right]\right)=0 }
\end{aligned}
$$

The general form of these equations can be written as

$$
F([x])=0,
$$

where 


$$
\begin{aligned}
{[\boldsymbol{x}] } & =\left[\begin{array}{lllllll}
\mathbf{V} & \mathbf{I}_{V} & \mathbf{I}_{L 2} & \mathbf{I}_{M} & \mathbf{I}_{L 3} & \mathbf{E}_{p} & y
\end{array}\right]^{T}, \text { the vector of unknowns, } \\
{[F] } & =\left[\begin{array}{lllllll}
f_{1} & f_{2} & f_{3} & f_{4} & f_{5} & f_{6} & f_{7}
\end{array}\right]^{T}, \text { the constraint equations } \\
{\left[\mathbf{I}_{u}\right] } & =\left[\begin{array}{llllll}
\mathbf{I}_{V} & \mathbf{I}_{L 2} & \mathbf{I}_{M} & \mathbf{I}_{L 3}
\end{array}\right]^{T} .
\end{aligned}
$$

and $\quad\left[\mathbf{I}_{V}\right]$ is the vector of currents from voltage sources,

$\left[\mathbf{I}_{L 2}\right]$ is the vector of single-phase $P Q$ (type-2) load currents,

$\left[\mathbf{I}_{M}\right]$ is the vector of machine currents,

$\left[\mathbf{I}_{L 3}\right]$ is the vector of static (type-3) load currents,

$\left[\mathbf{E}_{p}\right]$ is the vector of machine internal voltages,

$[y]$ is the vector of static load parameter $y$.

With the above development, the advantages of the branch-level component modelling approach compared with the traditional node-to-ground approaches can be seen more clearly. The major differences between the new multiphase load flow technique and the existing techniques (e.g. [18]) are summarized in Table 3.1 .

\subsubsection{Solution of Load Flow Equations}

Equation (3.33) is a set of nonlinear algebraic equations, which must be solved iteratively. Experience has shown that the Newton-Raphson method is probably the best method for conventional load flow studies [21]. It has also been chosen for the solution of Eq. (3.33). Rectangular coordinates are used here to separate the complex variables and equations into real form. Besides its simplicity, the rectangular representation has other advantages. For example, if there are no PV and PQ constraints, the Jacobian matrix becomes constant. The solution is then equivalent to the direct solution of the linear problem $[Y][\mathbf{V}]=[\mathbf{I}]$. 
Table 3.1: Comparison between proposed and existing load flow techniques

\begin{tabular}{|c|c|c|}
\hline TECHNIQUES & PROPOSED TECHNIQUE & EXISTING TECHNIQUES \\
\hline $\begin{array}{l}\text { PV Machine } \\
\text { PQ Machine }\end{array}$ & $\begin{array}{l}\text { 1. load flow constraints } \\
\text { specified } \\
\text { 2. arbitrary connections } \\
\text { (delta or wye) } \\
\text { 3. branch equations } \\
\text { as above } \\
\text { as above (new model) }\end{array}$ & $\begin{array}{l}\text { 1. no load flow constraints } \\
\text { specified } \\
\text { 2. grounded-wye connection } \\
\text { only } \\
\text { 3. node equations } \\
\text { as above } \\
\text { not available (impossible } \\
\text { to include unbalance effects) }\end{array}$ \\
\hline $\begin{array}{l}\text { Type-2 Load } \\
\text { Type-3 Load } \\
\text { Type- } 4 \text { Load }\end{array}$ & $\begin{array}{l}\text { as above } \\
\text { as above (new model) } \\
\text { as above (new model) }\end{array}$ & $\begin{array}{l}\text { phase to ground only } \\
\text { not available } \\
\text { not available }\end{array}$ \\
\hline $\begin{array}{l}\text { Voltage Sources } \\
\text { Current Sources }\end{array}$ & $\begin{array}{l}\text { as above } \\
\text { as above }\end{array}$ & $\begin{array}{l}\text { N/A (can be included) } \\
\text { N/A (can be included) }\end{array}$ \\
\hline $\begin{array}{l}\text { Load Flow } \\
\text { Equation }\end{array}$ & $\begin{array}{l}\text { formed by simply grouping } \\
\text { the above equations with } \\
\text { the multiphase }[Y] \text {-matrix } \\
\text { equation }\end{array}$ & $\begin{array}{l}\text { Real/reactive power equations } \\
\text { for each node are formed first. } \\
\text { Power constraints are then } \\
\text { specified for each node. }\end{array}$ \\
\hline Characteristic & $\begin{array}{l}\text { Load flow constraints } \\
\text { are the property of } \\
\text { network components }\end{array}$ & $\begin{array}{l}\text { Load flow constraints } \\
\text { are the property of } \\
\text { network nodes. }\end{array}$ \\
\hline
\end{tabular}

With the Newton-Raphson method, the system of linear equations

$$
\left[J_{i}\right]\left[\Delta x_{i}\right]=-\left[\Delta F\left(x_{i}\right)\right]
$$

is solved in each iteration step, and the variables are then updated with

$$
\left[x_{i+1}\right]=\left[x_{i}\right]+\left[\Delta x_{i}\right]
$$

where $\quad i$ is the iteration number,

$\left[J_{i}\right]$ the Jacobian matrix, $\left[\Delta F\left(x_{i}\right)\right]$ the residual vector. 
With Eqs. (3.26) to (3.32), Eq. (3.34) becomes

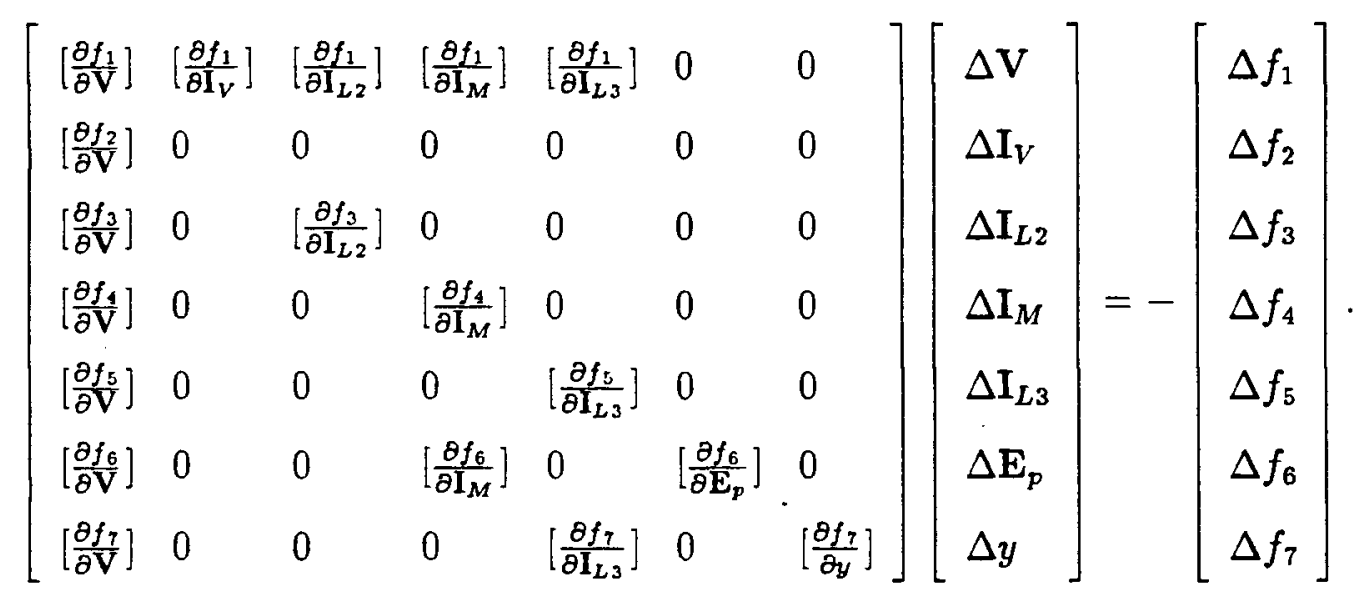

For the above equation, all the components in $[\Delta x]$ and $[\Delta F]$ are subvectors. Each element of these subvectors is further separated into real and imaginary parts, as following examples:

$$
\begin{aligned}
& {[\mathbf{V}]=\left[\begin{array}{llllll}
V_{R-1} & V_{I-1} & V_{R-2} & V_{I-2} & \ldots & V_{I-(\text { number of nodes })}
\end{array}\right]^{T}} \\
& {\left[f_{2}\right]=\left[\begin{array}{llllll}
f_{2 R-1} & f_{2 I-1} & f_{2 R-2} & f_{2 I-2} & \ldots & f_{2 I \text {-(number of voltage sources) }}
\end{array}\right]^{T}}
\end{aligned}
$$

where the subscripts $R$ and $I$ indicate real and imaginary part respectively. The submatrices in the first two rows of the large Jacobian matrix are constant matrices. In particular, $\left[\frac{\partial f_{1}}{\partial V}\right]$ is the node admittance matrix arranged in a real matrix form.

The procedure for obtaining the submatrices is the same as in conventional load flow techniques. Once the Jacobian matrix is obtained, Eq. (3.34) is solved by Gauss elimination with sparsity techniques. The largest component in the residual vector is used to test for convergence.

\subsection{Initialization of the Iterative Process}

Choosing an initial guess $\left[x_{0}\right]$ for the iterations is more complicated in the multiphase case. The traditional initialization technique, which uses 1.0 per-unit node voltage magnitudes 
with respective $120^{\circ}$ phase shifts among phases $a, b$, and $c$, becomes unreliable if there are phase shifting effects through wye-delta transformer connections. Since the convergence of the Newton-Raphson method is sensitive to the initial guess, a special initialization procedure is used before entering the iteration loop.

The procedure is based on the observation that the load flow equations become linear if there are no PV or PQ constraints. To approximate the network this way, the components with $\mathrm{PQ}$ and $\mathrm{PV}$ constraints are modified as follows:

(a) Machines with PV and PQ constraints and rotating machine loads are represented as admittance matrices of very small magnitude. This approximates open-circuit conditions.

(b) Other loads are represented as known admittances $y$ whose values are determined from

$$
y|\mathbf{V}|^{2}=(P-j Q)_{\text {specified }}
$$

for the single-phase PQ loads, and

$$
y\left([T][K][T]^{H}\right)|\mathbf{V}|^{2}=(P-j Q)_{\text {specified }}
$$

for three-phase static loads. The voltage magnitude $|\mathbf{V}|$ is estimated to be equal to the user-supplied rated voltage of the load.

With these approximations, the load flow solution becomes linear. Rather than writing a separate algorithm for this initialization, the normal Newton-Raphson algorithm is used with zero initial values, and the linear estimate $\left[x_{1}\right]$ is obtained in one iteration. This becomes the starting point for the following load flow iterations. 


\subsection{Frequency Scan Extension}

The extension of the multiphase load flow program to provide the capability of performing frequency scans is needed for the ultimate multiphase harmonic analysis. To implement this extension, it is first necessary to define how the machines and loads respond to harmonics.

\subsubsection{Harmonic Models of Network Components}

(1) Machines. As a first approximation, it is assumed that machines do not produce harmonics ${ }^{2}$. For harmonic frequencies they can be modelled as known admittance matrices, as suggested in reference [22]:

$$
\begin{gathered}
Y_{h-p}=Y_{h-n}=1 /\left(j h X_{n 1}\right) \\
Y_{h-o}=1 /\left(j h X_{\mathrm{o} 1}\right),
\end{gathered}
$$

where $\mathrm{h}$ is the harmonic order, and $X_{n 1}$ and $X_{o 1}$ are the negative and zero sequence reactances at fundamental frequency, respectively. The excitation voltage is simply zero for $h>1$. A similar model is used for rotating machine load.

(2) Loads. The performance of the load under the combined effects of unbalanced and harmonic conditions is usually not well known. Using the load modelling techniques of reference [29], a multiphase load can be modelled as a combination of lumped $R, L, C$ elements. If the test data needed for this representation is not available, the recommendations of reference [22] can be used, as has been done in Equation (2.9) of Chapter 2. Using these approximations, the individual-load of Figure 3.1(c) is replaced by $Z_{\text {load }-h}$, and the static load of Figure 3.1(d) is represented as

$$
Z_{h-p}=Z_{h-n}=Z_{\text {load }-h}
$$

\footnotetext{
${ }^{2}$ Detailed analysis on the machine's harmonic behaviour will be presented in Chapter 5 .
} 


$$
Z_{h-0}=r_{h}^{-1} Z_{h-p}
$$

where $r_{h}$ is the user supplied positive to zero sequence impedance ratio of the load at the given frequency. These sequence parameters are then transformed into phase quantities.

3. Transmission lines. Transmission lines can be represented as exact multiphase $\pi$ equivalent circuits calculated at the considered harmonic frequency [12].

\subsubsection{Solution Technique}

With the various system components represented at each harmonic frequency, the problem is also formulated as a multiphase load flow problem of Equation (3.33) and solved by the Newton-Raphson method. However, since the machines and loads are represented as constant impedances at harmonic frequencies, the problem is linear and iterations are therefore not required.

\subsection{Case Studies}

The performance of the multiphase load flow program has been investigated with four test systems. Comparisons between analytical and measured results were made for one system.

\subsubsection{Field Measurement Comparisons}

Field measurements for an unbalanced railway load case were made available by B.C. Hydro. The problem involves a single-phase railway load connected to a $230 \mathrm{kV}$ substation as shown in Figure 3.2. The network within the dashed box is a three-phase diagram of the electric system of the railway. In this arrangement, only phases A and B supply electric power to the railway through a single-phase $230 \mathrm{kV} / 50 \mathrm{kV}$ transformer. Since the railway load (around 30MVA) accounts for 30 percent of the total Tumbler Ridge 
(TLR) substation load, the load unbalance can be quite severe in some cases. As a result, complaints about motor trippings, excessive voltage unbalances, and capacitor banks damage have been reported [27, 32].

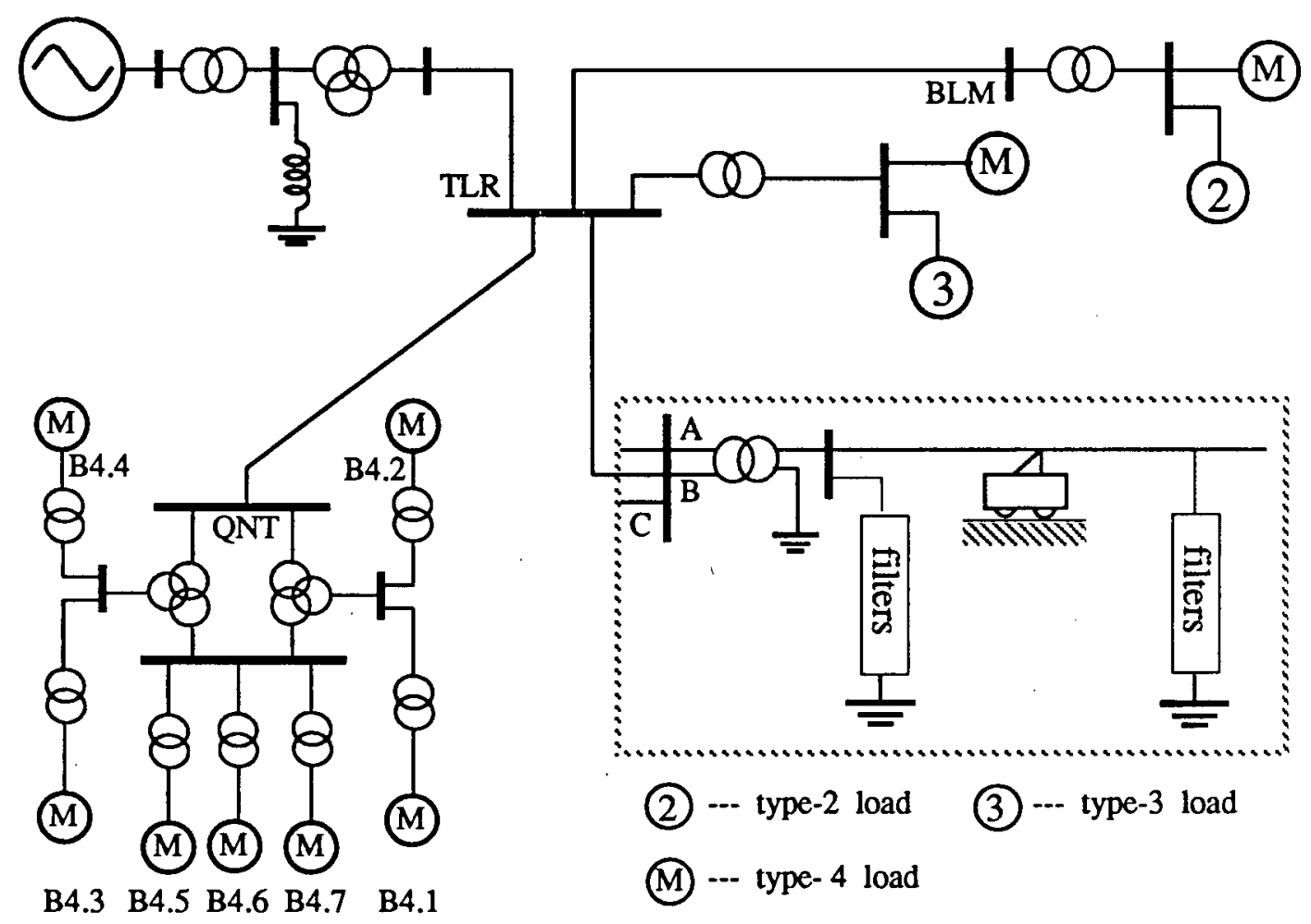

Figure 3.2: The B.C. Hydro TLR area network with single-phase railway load.

Field measurements were taken to identify the problems associated with the TLR bus voltage unbalance and the railway operations [27]. To study this system, the network model of Figure 3.2 was developed based on reference [32] (see Appendix E). In this model, all motor loads are represented as type-4 load model. The sequence impedances of the motors are estimated from reference [33]. Other loads are modelled as either type-2 or type-3, according to the observations and recommendations of reference [27]. 
Figure 3.3 shows the measured and calculated voltage unbalance at the TLR substation. The unbalance is shown as the ratio of negative to positive sequence voltages and is determined for different railway loading conditions. For the purpose of comparison, results were also calculated using the load models of existing techniques, that is, with all three-phase loads represented as three individual-phase node-to-ground PQ loads.

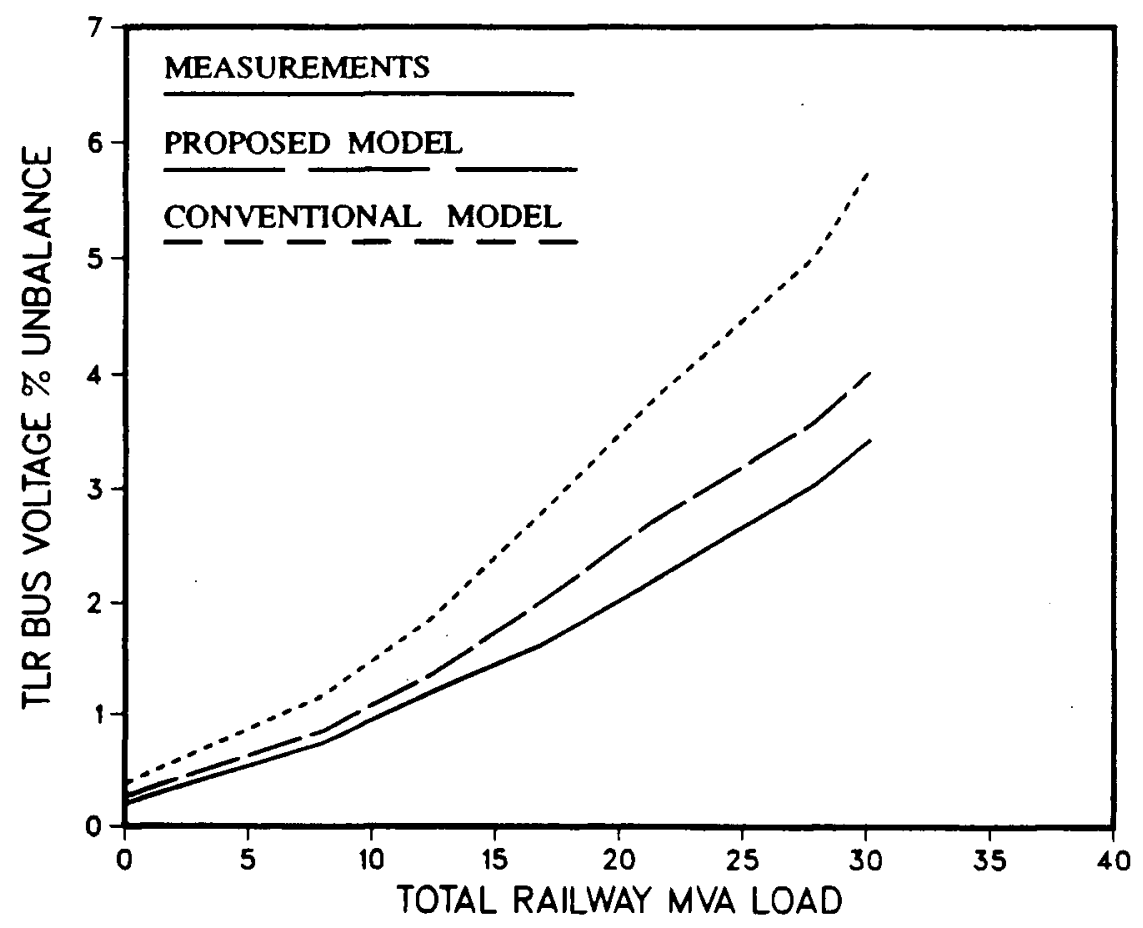

Figure 3.3: TLR bus voltage unbalance as a function of railway load.

From the results shown in Figure 3.3, it can be seen that the proposed load modelling gives reasonable results, while the conventional $P Q$ modelling gives large errors. The accuracy of both modelling techniques can further be assessed by observing the unbalance of the current flowing from the TLR bus to the QNT and BLM busses. This current unbalance is shown in Figure 3.4. The large errors with the conventional PQ modelling may lead to erroneous conclusions regarding, for instance, the identification of motor 
trippings.

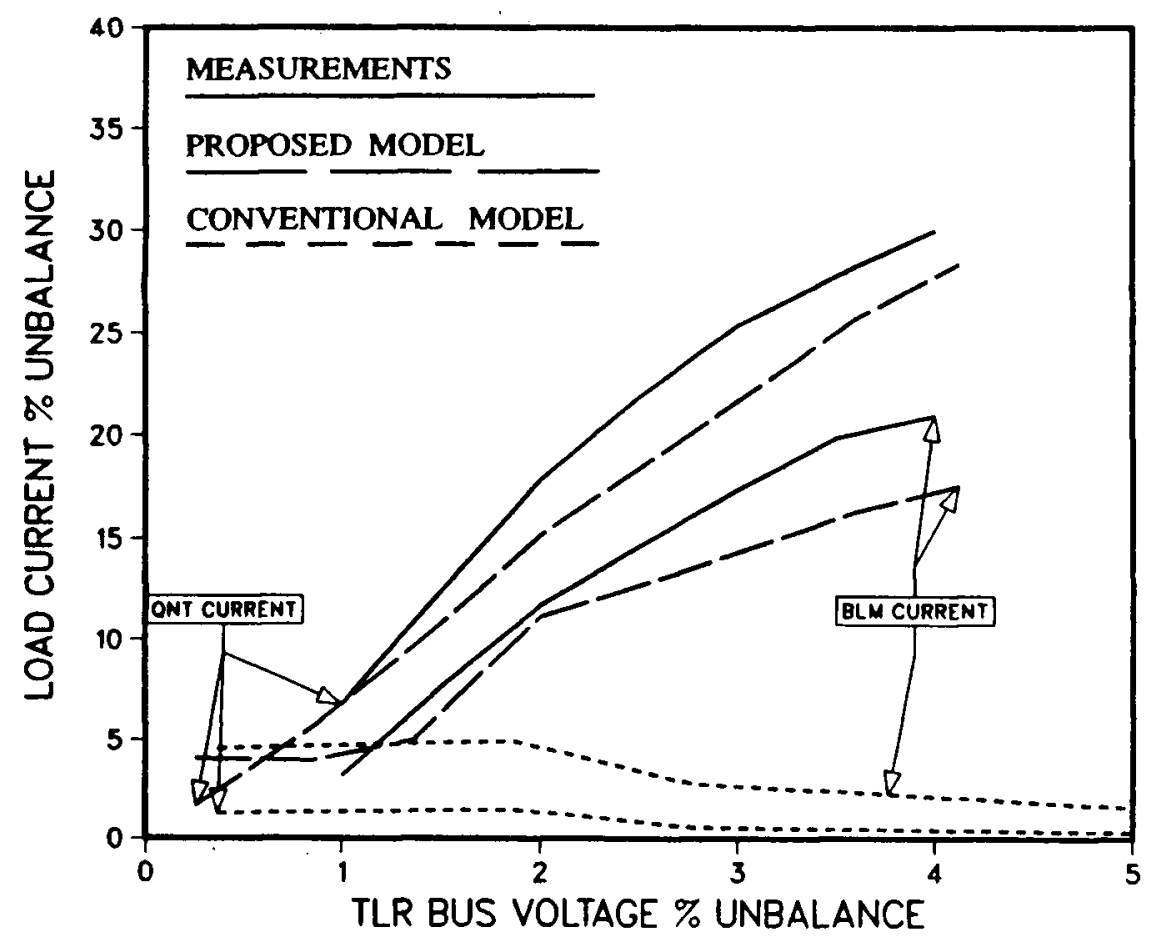

Figure 3.4: Bus current unbalance as a function of TLR bus voltage unbalance.

The reason for the poor results obtained with the conventional modelling techniques is that their load model assumes equal positive and negative sequence impedances for the induction motors, whereas in actual motors, the negative sequence impedance is much smaller than the positive sequence impedance. As a result, the motors are able to sink some of the negative sequence currents from the unbalanced loads. Type- 4 modelling of the motor load takes this sequence inequality factor into account and more realistic simulation results are obtained. There is still a discrepancy in the simulation results of Figure 3.3 and Figure 3.4 with respect to the measured values. Possible reasons for this discrepancy are the lack of actual data for the motor parameters and the limited knowledge of the loading conditions at the time the measurements were taken. 


\subsubsection{Unbalances in the B.C. Hydro Railway Case}

The program is then used to investigate some of the reported unbalance problems, in particular the problem of motor trippings, in the B.C. Hydro system described in the previous section.

Simulation results were obtained for current unbalances in the major motors of the system. Table 3.2 shows the unbalance factors of motor currents for three voltage unbalance levels at the TLR bus. As indicated in Table 3.2, the current unbalance of some motors can go beyond the usual $20 \%$ relay setting when the TLR bus experiences $2 \%$ to $3 \%$ voltage unbalances. This observation is consistent with the actual situation reported in reference [27]. It can also be observed that the degree of current unbalance of small motors is higher than that of large motors.

Table 3.2: Percentage unbalance of motor currents.

\begin{tabular}{|c|c|c|c|c|c|c|c|c|}
\hline \multicolumn{2}{|c|}{ Motor location } & 4.1 & $\overline{\mathrm{B} 4.2}$ & $\overline{\mathrm{B} 4.3}$ & 4.4 & 4.5 & 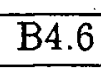 & B4. \\
\hline \multicolumn{2}{|c|}{ Motor rating(hp) } & & $\overline{300}$ & 0000 & 5700 & 1000 & & \\
\hline \multirow{4}{*}{$\begin{array}{l}\text { TLR bus } \\
\text { voltage } \\
\text { unbalance } \\
(\%)\end{array}$} & & \multicolumn{7}{|c|}{$\overline{\text { Motor current unbalance (\%) }}$} \\
\hline & & & & & & & & \\
\hline & &. \pm & & & & & & \\
\hline & & 9.07 & 2.2 & 13 & 4. & 0.2 & 2.0 & 5. \\
\hline
\end{tabular}

In order to reduce the network voltage and current unbalance, the following operation scenarios were investigated:

1. Connect the railway load between phases $B$ and $C$, or $A$ and $C$, instead of the present connection between phases $\mathrm{A}$ and $\mathrm{B}$.

2. Change the power factor of the total railway load.

It was found in the first scenario that for a railway load of $30.15 \mathrm{MVA}$, the voltage unbalance at the TLR bus was $4.12 \%$ for the load connected between phases A and B 
(actual connection), $4.76 \%$ for the B-C connection, and $3.97 \%$ for the A-C connection. Therefore, the system unbalance might be slightly improved by simply switching the load to phases $\mathrm{A}$ and $\mathrm{C}$.

For the second scenario, the change in the TLR bus voltage unbalance as a function of the total railway load power factor is listed in Table 3.3 for a total railway load of 22MVA. The results in this table indicate that the leading power factor can reduce voltage unbalance to some degree.

Table 3.3: TLR percentage voltage unbalance with power factors.

\begin{tabular}{|l|ccccccc|}
\hline Power factor & $0.7^{*}$ & $0.8^{*}$ & $0.9^{*}$ & 1.0 & 0.9 & 0.8 & 0.7 \\
\hline TLR voltage unbalance(\%) & 2.410 & 2.443 & 2.501 & 2.694 & 2.938 & 3.045 & 3.123 \\
\hline
\end{tabular}

* - Leading power factor.

\subsubsection{Frequency Scan Analysis of the TLR system}

Measurements on the TLR bus in the B.C. Hydro railway system showed significant negative sequence harmonics in the phase voltages [27]. It was also observed that the 5th harmonic in the voltages was insensitive to the change of railway load. Frequency scan analysis were performed with the program in order to verify these measurements. Simulations were run by injecting one ampere of current of different frequencies at the locomotive bus and monitoring the TLR bus harmonic voltages. These results are shown in Figure 3.5.

Even though direct frequency scan measurements were not available for comparison, significant correlations between the plots and the field observations were noted. The calculated results were also consistent with those of reference [32]. The computed results suggest 
1. The locomotive can cause more distortion on the negative sequence harmonics than on the positive sequence ones.

2. The 5 th harmonic distortion at the TLR bus is prevented by the 5 th harmonic filter of the railway subsystem.

3. Increase of the railway load may not be a problem from the locomotive harmonic generation point of view, since the railway subsystem sinks most of its harmonics due to installed filters.

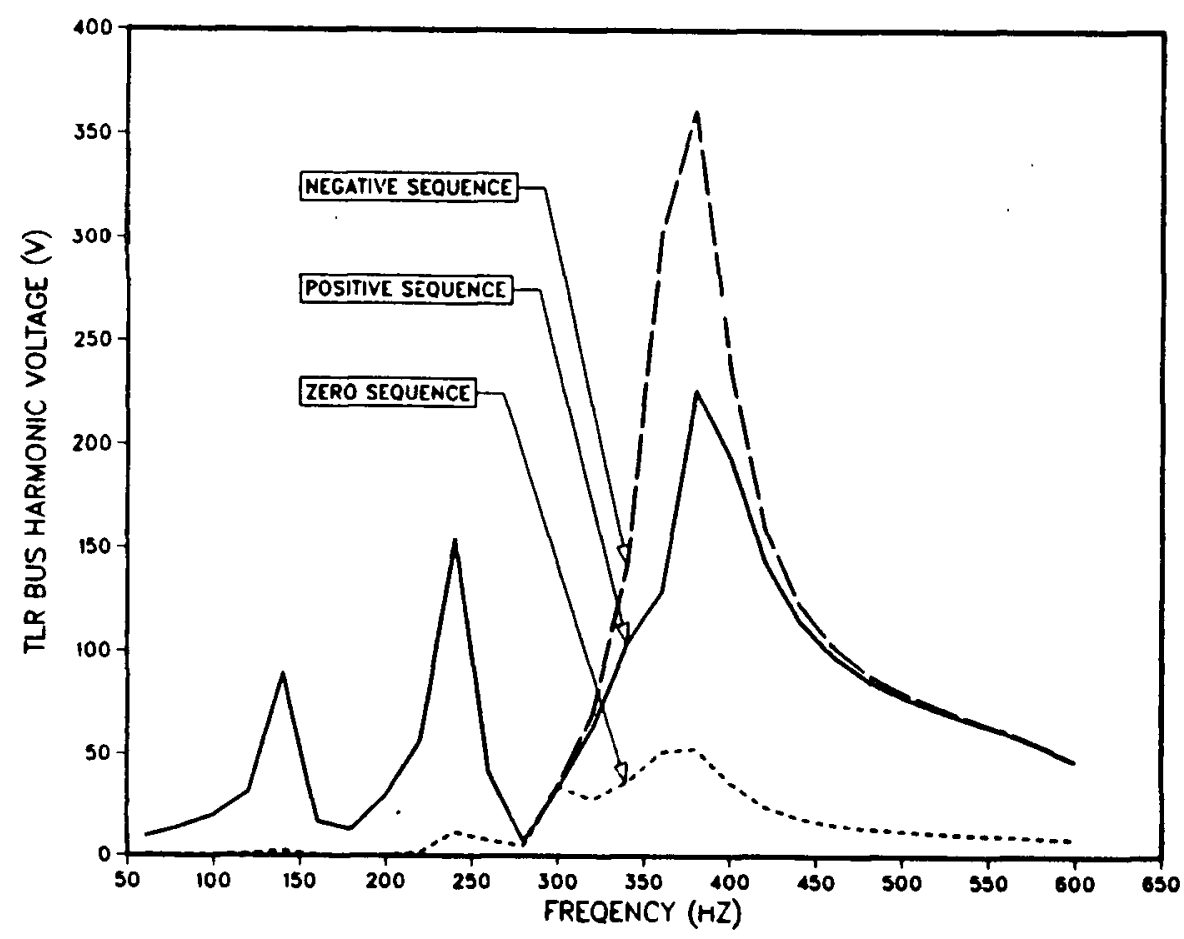

Figure 3.5: Frequency scan of the TLR system.

\subsubsection{Observations on the Convergence Behaviour}

The numerical performance of the program has been investigated with four test systems: 
1. The B.C. Hydro's TLR substation railway load case described above (data are listed in Appendix E).

2. The BPA three-phase load-flow test case for EMTP initialization [12].

3. The 7 bus simplified Southern Alberta system case (Figure 3.6).

4. The IEEE 118-bus single-phase load-flow test system [26].

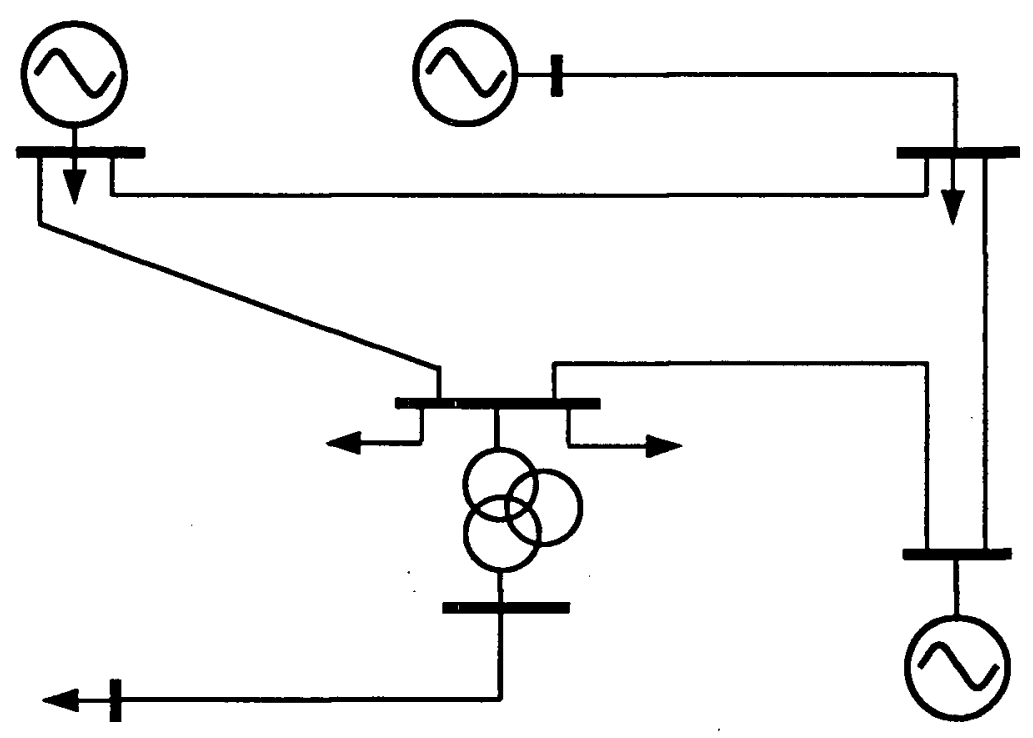

Figure 3.6: Single-phase diagram of test system 3.

For the test systems above, Table 3.4 lists the maximum relative residuals in each iteration and the total number of iterations and CPU times needed to converge to the solution.

It is noted in Table 3.4 that the relative residuals in the first iteration are around 100 percent. This is the typical value experienced in conventional single-phase load flow solutions. It indicates that the starting point estimation process described in Section 3.5 
for the multiphase program has effectively provided reasonable starting point conditions. Further observation suggests that the method exhibits quadratic convergence behaviour for both the balanced and unbalanced cases, which is what is expected for the Newtontype methods. It is also noted that the method needs more iterations for the unbalanced cases than for the balanced ones. Respective tests indicate that the extra iterations in the unbalanced cases is probably due to the assumption of balanced properties in some parts of the system during the estimation of the starting solution point (e.g. Eq. (3.37)).

Table 3.4: Convergence behaviour of the multiphase load flow technique.

\begin{tabular}{l|cccccc|}
\hline Iter.\# & sys-1 & sys-2** & sys-2 & sys-3** & sys-3 & sys-4 \\
\hline \hline 1 & 1.00000 & 0.27985 & 0.23528 & 1.38776 & 1.20530 & 1.00000 \\
2 & 0.20794 & 0.00050 & 0.04566 & 0.06654 & 1.81466 & 1.06509 \\
3 & 0.00821 & 0.00001 & 0.00468 & 0.00039 & 0.38840 & 0.14139 \\
4 & 0.00004 & & 0.00009 & 0.00000 & 0.05428 & 0.00650 \\
5 & & & & & 0.00184 & 0.00009 \\
6 & & & & & & 0.00000 \\
\hline \hline CPU(s) & 36.50 & 0.97 & 1.11 & 10.49 & 13.65 & 276.24 \\
\hline NODE & 64 & 13 & 13 & 33 & 33 & 118 \\
BRAN & 163 & 18 & 18 & 60 & 60 & 289 \\
JACB & 214 & 38 & 38 & 124 & 124 & 566 \\
\hline * - Railway load 30.15MVA. & & & \\
** - Balanced operating conditions. \\
NODE - Total number of nodes. \\
BRAN - Total number of branches. \\
JACB - Dimension of Jacobian matrix.
\end{tabular}

The convergence of the single-phase IEEE test case was compared with that of a dedicated single-phase load flow program. In these single-phase comparisons, the program had the same convergence rate as the dedicated single-phase load flow program in spite of the program's larger Jacobian matrix to include the generator models. 
In addition to these reported cases, the program was also tested using a number of fictitious systems with irregular connections. The program exhibited fast convergence in those cases as well.

\subsection{Summary}

A multiphase load flow program has been developed in this chapter. This program is essentially a general purpose steady-state network analysis program with built-in multiphase load flow constrained components. Compared with the previous work, the program provides a wide range of load models for unbalanced load flow analysis. With the concept of branch-level load flow constraints, the program possesses the capability to model any possible network component connections and constraint specifications. As well, it has exhibited good convergence performance with the utilization of the Newton-Raphson method and a reliable starting point estimation procedure.

As a tool for the steady-state analysis of unbalanced power systems, the multiphase program has been successfully tested with a number of cases. The usefulness and correctness of the technique and its load models were confirmed by the comparisons of the computed results with field measurements.

For the objective of multiphase harmonic analysis, the program has been extended with frequency scan capability. Moreover, the three functions of the program, initialization, load flow solution and frequency scan, are all configured in one solution structure, namely, the Newton-Raphson solution process with or without iterations. Thus it has formed a comprehensive base needed for the multiphase harmonic load flow technique. 


\section{Chapter 4}

\section{Multiphase Harmonic Load Flow Technique - The Basic Form}

With reference to the single-phase method of Chapter 2, the second step towards developing the multiphase harmonic load flow technique is to derive frequency-domain models of the nonlinear elements. There is no standard solution to this problem, however, due to the variety of nonlinear characteristics. The resultant models may also take many different forms ranging from simple current sources to multiphase Norton equivalent circuits.

Once the frequency-domain models are obtained, they are interfaced with the multiphase network load flow solution and frequency scan process developed in Chapter 3 . The harmonic load flows can then be solved. The purpose of this chapter is to present the general procedure followed in the multiphase harmonic load flow (MHLF) technique for the analysis of any particular nonlinear element. It is illustrated with the harmonic analysis of nonlinear inductors.

\subsection{Frequency-domain Models of the Nonlinear Inductors}

As just mentioned, there are many approaches to derive the frequency-domain models of nonlinear elements. For the modelling of nonlinear inductors, at least two methods have been published. These methods proposed the current source model [14] and the harmonically-coupled impedance model [34]. The modelling technique employed in this work is similar to reference [14], but it yields a harmonically-decoupled Norton equivalent circuit. Time-domain simulation and numerical Fourier analysis are used in this 
approach. Since nonlinearities can more easily be dealt with by time-domain techniques, the approach is somewhat of general nature, and will also be used in later chapters for the model development of synchronous machines and thyristor-controlled reactors.

The current-flux relationship of a nonlinear inductor can generally be expressed as a nonlinear function:

$$
i=S(\lambda) \text {. }
$$

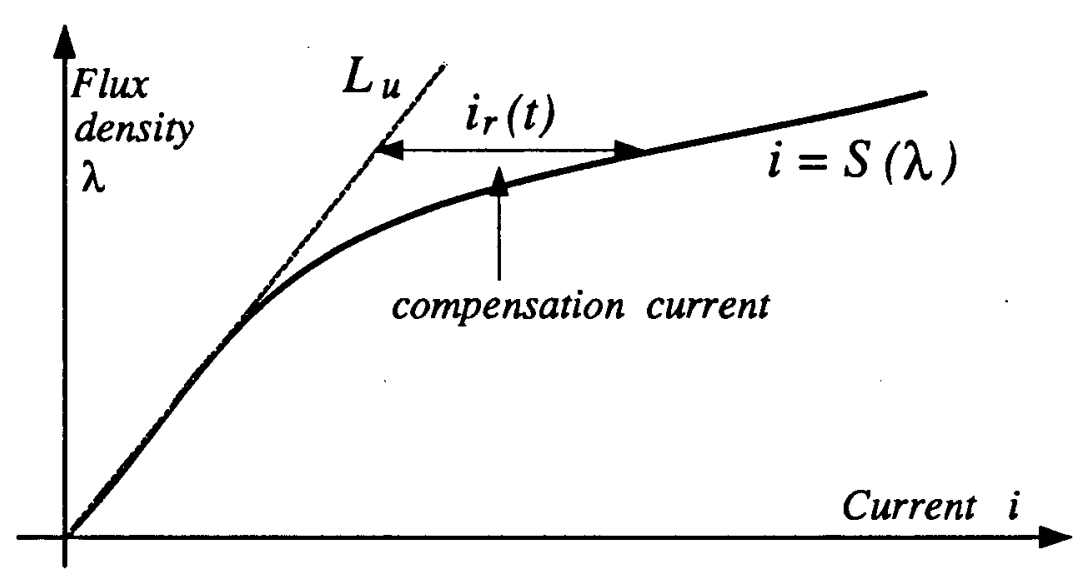

Figure 4.1: Characteristics of a nonlinear inductor.

This characteristic is shown in Figure 4.1, and is assumed to be symmetric with respect to the origin. It is generally true that the unsaturated inductance $L_{u}$ can represent the nonlinear inductor well at fundamental frequency and other frequencies if no saturation is to be modelled. This inductance is therefore adopted as the equivalent inductance of the Norton equivalent circuit for the nonlinear inductor. The difference between the current absorbed in $L_{u}$ and the actual current from Eq. (4.1) become the parallel current 
sources in the Norton equivalent circuit, as follows:

$$
\begin{aligned}
i(t) & =S(\lambda(t))-\lambda(t) / L_{u}+\lambda(t) / L_{u} \\
& =-i_{r}(t)+\lambda(t) / L_{u}
\end{aligned}
$$

where

$$
i_{r}(t)=\lambda(t) / L_{u}-S(\lambda(t))
$$

is the parallel current source needed to compensate for the nonlinear effects. Since $d \lambda / d t=v$, Eq. (4.2) can be further expressed as

$$
L_{u} \frac{d}{d t}\left[i(t)+i_{r}(t)\right]=v(t)
$$

where $v(t)$ is the terminal voltage of the nonlinear inductor. It is straightforward to find that the above equation describes a linear Norton equivalent circuit of the inductor, as shown in Figure 4.2 .
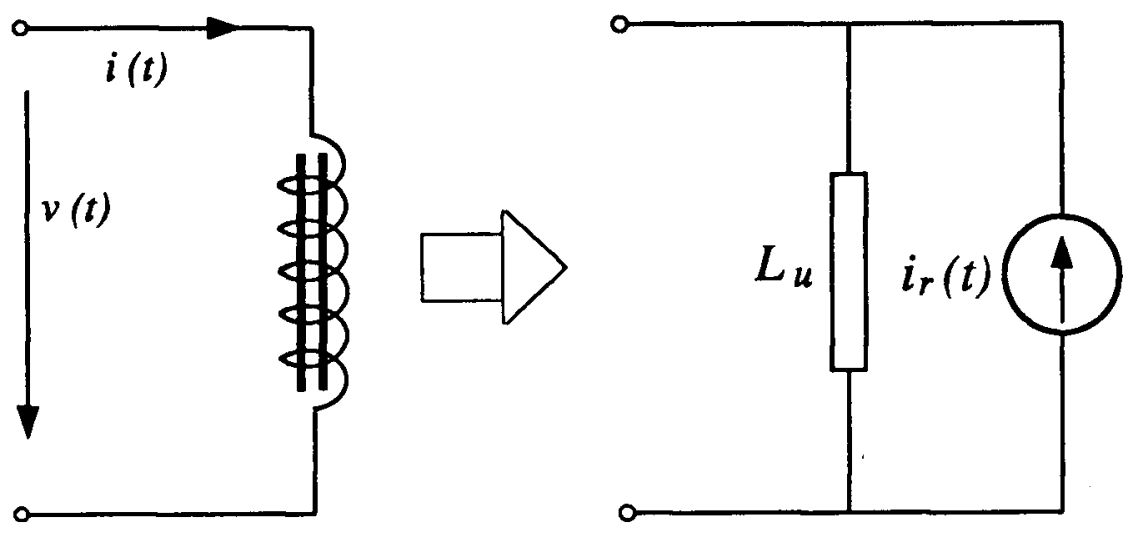

Figure 4.2: Norton equivalent circuit of a nonlinear inductor.

To complete this model, $i_{r}(t)$ must be determined. Assume that the terminal voltage 
$v(t)$ is known and can be expressed as the harmonic series

$$
v(t)=\sum_{h=1}^{n} \sqrt{2}\left|\mathbf{V}_{h}\right| \cos \left(h \omega t+\phi_{h}\right)
$$

where $n$ is the number of harmonics of interest. Accordingly, the flux $\lambda(t)$ is given by

$$
\lambda(t)=\sum_{h=1}^{n}(h \omega)^{-1} \sqrt{2}\left|\mathbf{V}_{h}\right| \sin \left(h \omega t+\phi_{h}\right) .
$$

With this known flux waveform, current $i_{r}(t)$ can be computed point-by-point from equation (4.3), and then analyzed by numerical Fourier analysis. This produces the required harmonic expression of the current:

$$
i_{\tau}(t)=\sum_{h=1}^{n} \sqrt{2}\left|\mathbf{I}_{h-e q}\right| \cos \left(h \omega t+\theta_{h}\right)
$$

In other words, with this approach a nonlinear inductor can always be represented in the frequency-domain as a Norton equivalent circuit of Figure 4.2: The harmonic coupling nature of the inductor is included in the known current source of the circuit. This current source is computed with a simple time-domain numerical process under the assumption of a known terminal voltage condition.

\subsection{Interface with the Multiphase Network Solution Process}

To compute the harmonic distributions caused by the nonlinear inductors, the network harmonic load flow conditions must be solved. The multiphase network solution technique developed in Chapter 3 can readily be used for this purpose. In this application, the Norton equivalent circuits representing nonlinear inductors are added into the network solution data base. A simple fundamental frequency solution and harmonic frequency scan will provide the harmonic load flow results.

It must be noted, however, that the current sources $i_{r}(t)$ of the Norton models are not known exactly beforehand. They can only be estimated from the terminal voltage 
conditions prior to the models being applied. An iterative process is therefore needed for the network solution part to obtain harmonic load flows with a certain accurate tolerance. This iterative process that forms the fundamental solution structure of the MHLF technique is now outlined as follows:

Step 1. Initialization.

Represent the nonlinear elements with simple linear models. For the nonlinear inductors, these initial models may be the Norton equivalent circuits with zero compensation current, i.e. $i_{r}(t)=0$.

The iteration numbers are initialized to zero.

Step 2. Network Solution.

Replace the nonlinear elements with their frequency-domain models in the network and solve the network equations by the technique described in Chapter 3 at the fundamental and the harmonic frequencies of interest.

Step 3. Frequency-domain Equivalent.

Compute the new harmonically-decoupled frequency-domain models for the nonlinear elements using the newly obtained network voltages and currents.

Step 4. Convergence Check.

If the obtained equivalent circuits are sufficiently close to the previous ones, a convergence criteria has been reached. The iteration is then stopped.

Otherwise, the equivalent circuits and the iteration numbers are updated, and the process is redirected to Step 2 .

This is the basic form of the proposed multiphase harmonic load flow technique. It is a very general algorithm and can be applied to any nonlinear element, as long as the 
element can be represented by a frequency-domain Norton equivalent circuit. The flow chart of this harmonic iteration process is shown in Figure 4.3. In the figure, process NETWORK_SOLUTION is a subroutine to form the multiphase network equations and to solve them with conditions such as load flow constraints, harmonic excitations and unbalances. The initialization of the entire harmonic load flow iteration is also described in the figure. For the harmonic analysis of nonlinear inductors, control characteristics need not be considered. However, the control characteristics of other nonlinear elements can be included, as will be discussed in the next two chapters.

\subsection{Test Results}

The performance of the MHLF technique has been investigated with recorded field results from a Brazilian utility company (CEMIG) [10]. The network consists of three generator sets connected through two sets of three-winding transformers feeding into a shunt compensated $345 \mathrm{kV}$ overhead transmission line (see Figure 4.4). In this study, the nonlinearity is mainly due to the presence of the magnetizing impedances of the stepup transformers. The corresponding waveforms are computed by the MHLF technique. The obtained results are then compared with field test records as well as the EMTP simulations.

\subsubsection{Comparison with Field Test Results}

The field test data available to this work was obtained with balanced operating conditions. Even though the system is balanced, the flow of harmonics with orders $3,9,15$ etc. follows the 'zero sequence path' [35]. Since the zero sequence path can be different from the positive sequence network, the single-phase simulations that are normally based on the positive sequence components may yield incorrect solutions. As a result, three-phase simulation which has automatically taken the zero sequence components into account is 


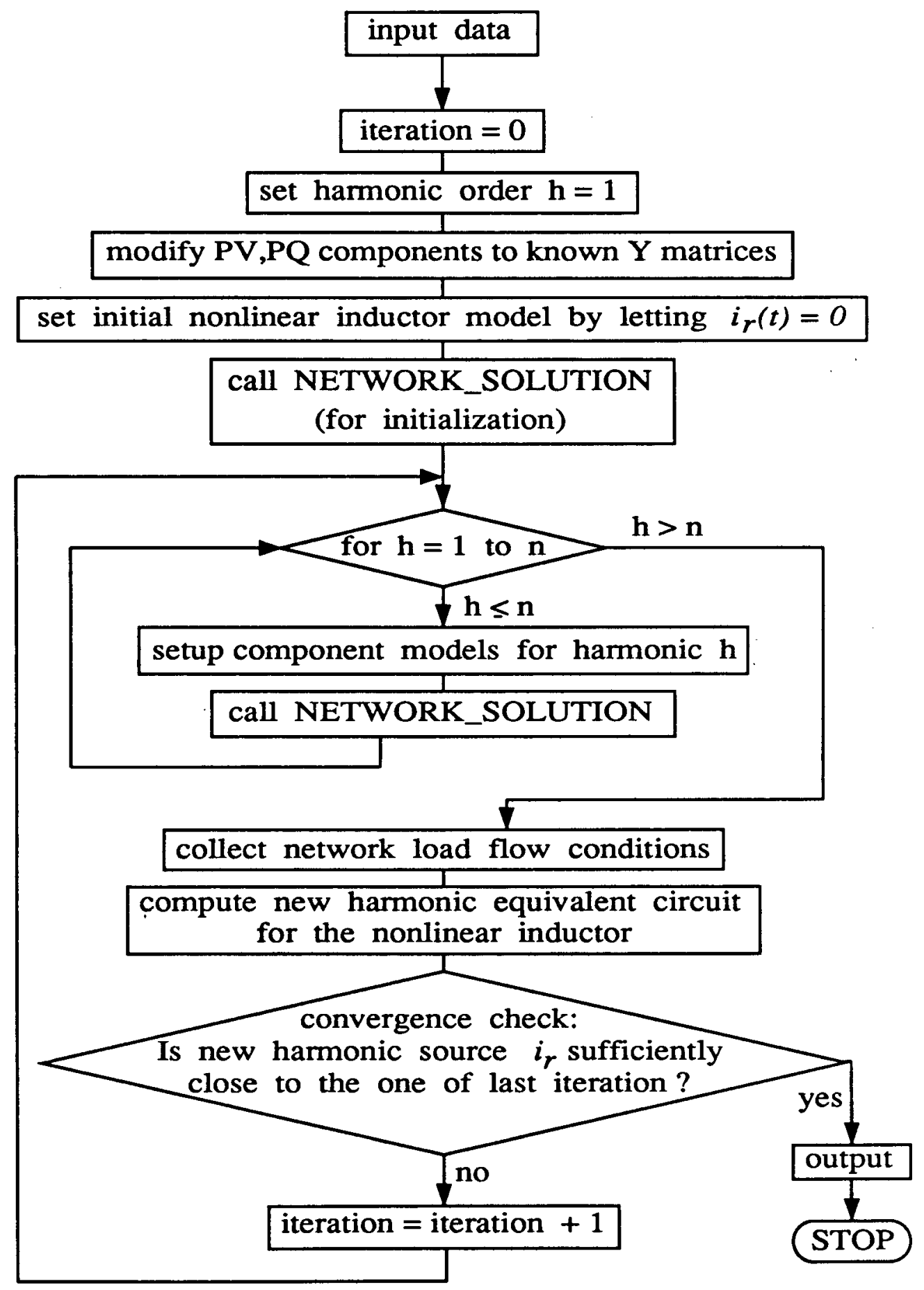

Figure 4.3: Flow chart of the MHLF technique. 


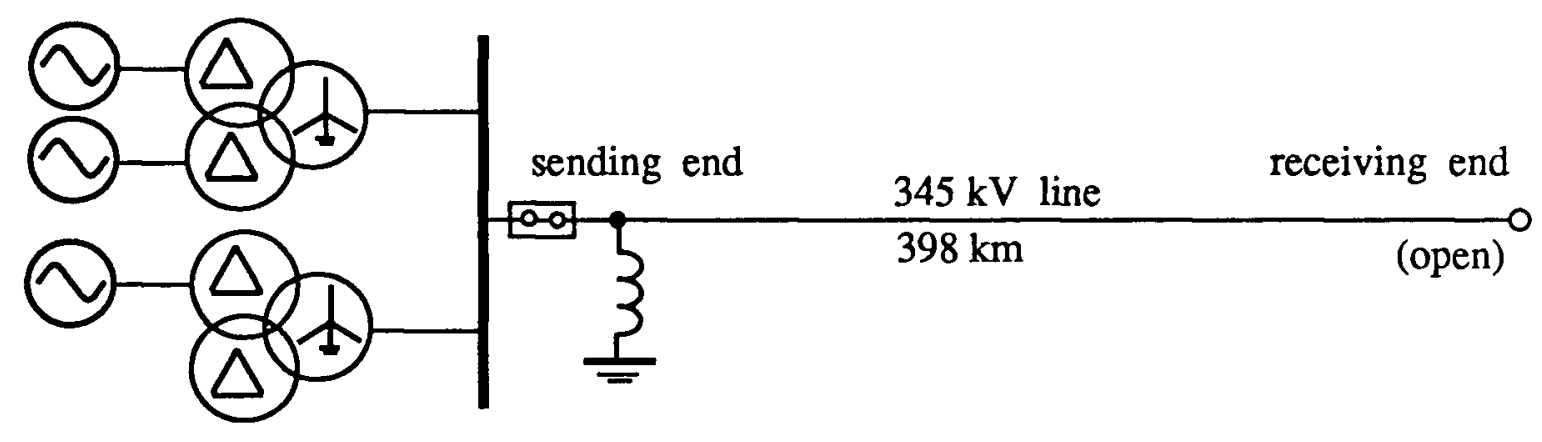

Figure 4.4: Single-phase diagram of test system CEMIG.

adopted. In the simulation, all three magnetizing branches of the step-up transformers are modelled as two-slope nonlinear inductors connected from phase to ground. The generators are represented by the equivalent machine model as derived from last chapter, The generator internal voltages are specified according to the given system conditions.

A comparison between the MHLF simulation and field test results is shown in Figure 4.5. The line to ground voltages of a certain phase at the sending and receiving end are compared. Similar results for the other two phases are also obtained except the $120^{\circ}$ phase difference, due to the balanced conditions. As shown by the good correlation with field measurements, the correctness of the MHLF technique is confirmed.

\subsubsection{Comparison with EMTP Simulation Results}

The MHLF technique is also compared with the EMTP transient simulation for an unbalanced test case. In this test case, the same CEMIG network is adopted but with the equivalent generator voltages modified into unbalanced voltage sources. It was found that the EMTP simulation which starts from a linear AC steady-state condition can build up 

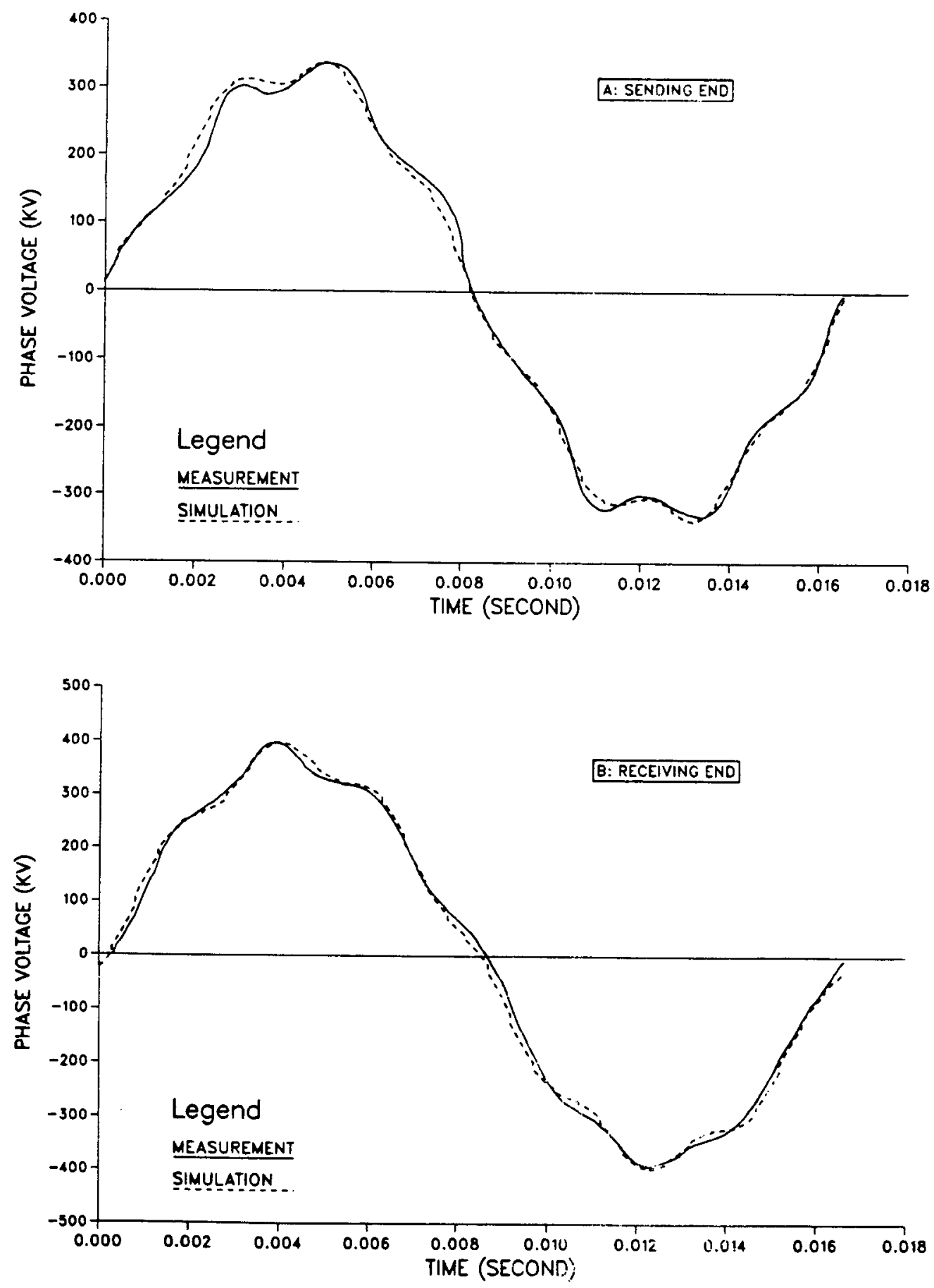

Figure 4.5: Comparison between MHLF and field test results. 
to the final waveforms in about 3 cycles in this case. Figure 4.6 shows the waveforms from these two completely different solution methods. Again, the correctness of the MHLF technique is confirmed with the close agreement between the two solution results. The results also indicate that the MHLF technique is applicable for EMTP initialization. This MHLF initialization is much more efficient than the present scheme in the EMTP. It can save a large amount of computer time especially when harmonic components take long time to build up to the final waveform.

\subsubsection{Analysis of Harmonic Unbalance}

To demonstrate the importance of the multiphase modelling in harmonic analysis, the sequence and unbalance of the three-phase harmonics obtained for the unbalanced test case were analyzed. This was done by evaluating the symmetrical components of the selected waveforms. The results for the harmonic voltages at the sending and receiving end of the line, and for the current at the generator are tabulated in Table 4.1.

It is well-known that, under balanced conditions, the $3 \mathrm{rd}, 9$ th and 15 th harmonics are of zero sequence type; while the 5 th and 11 th harmonics are of negative sequence. Accordingly, the results in Table 4.1 are normalized with respect to these sequence quantities. Examination of the table indicates that the 3rd and 9th harmonics are still dominantly in zero sequence, and the 5 th, 11 th harmonics in negative sequence, as footnoted in the table. However, due to the slight unbalance at the fundamental frequency, the components of all other sequences are present. It indicates the unbalance of harmonic components. As suggested by the normalized results of the table, such unbalances increase considerably with the harmonic orders. Figure 4.7 gives more clear illustration of this harmonic property. The figure shows the results of Table 4.1 in the form of bar chart. Based on these results, one may conclude: 


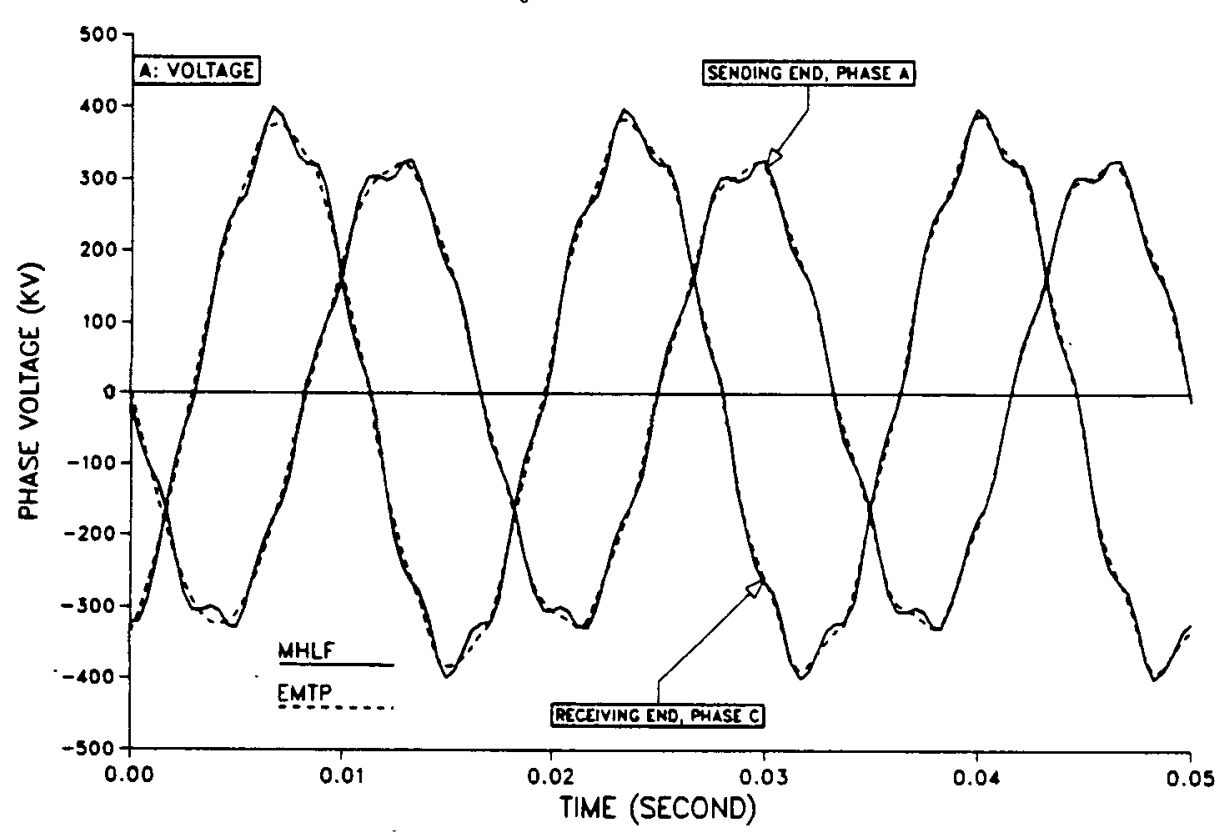

(a) phase $\mathrm{A}$ sending and phase $\mathrm{C}$ receiving end voltages

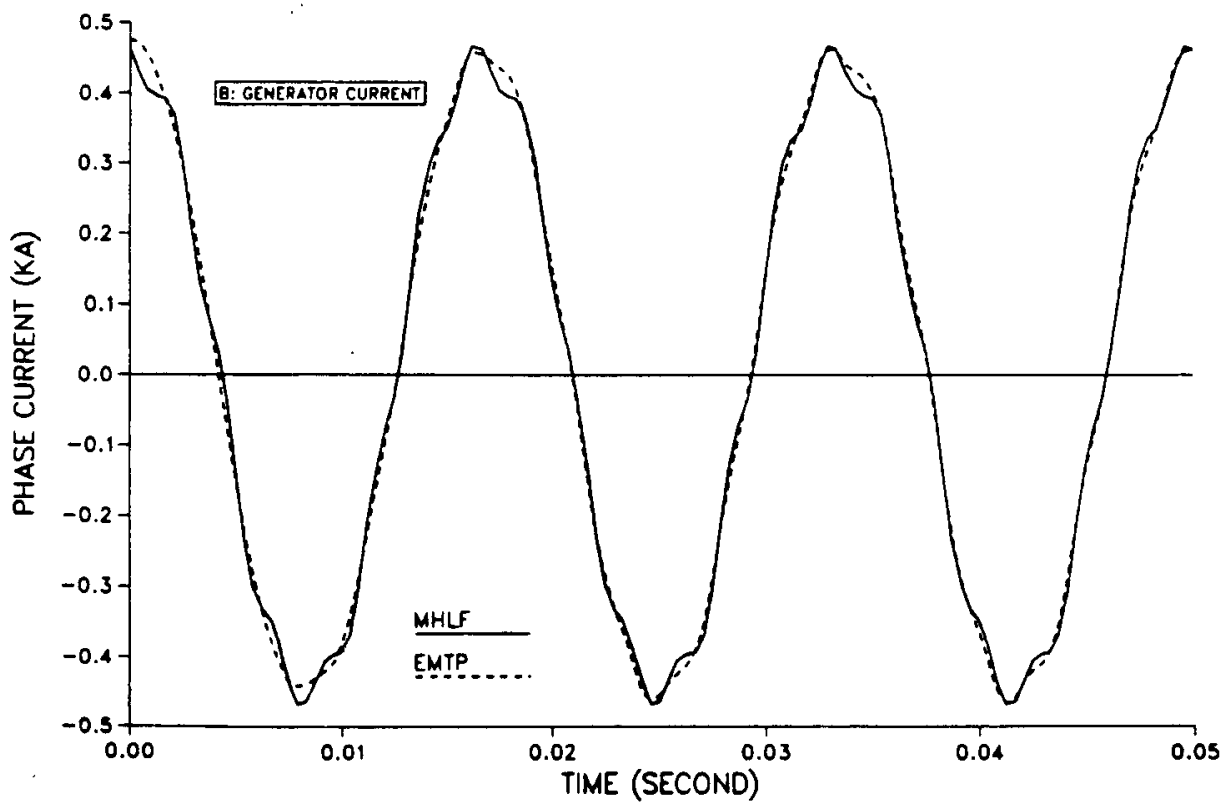

(b) generator phase A current

Figure 4.6: Comparison between MHLF and EMTP results. 
1. The $3 \mathrm{rd}, 9$ th and 15 th harmonics are predominantly in zero sequence. Whereas the 5 th and 11 th harmonics are in negative sequence. To study the distribution of such harmonics (even in balanced conditions), zero and negative sequence networks are needed. In the multiphase framework of the MHLF, sequence quantities are automatically and correctly represented.

2. A slight unbalance in the fundamental frequency components ( $2 \%$ in Table 4.1 ) can result in significant unbalance on the harmonic components. The degree of the harmonic unbalance generally increases with the harmonic orders.

Table 4.1: Harmonic contents of the unbalanced test case.

\begin{tabular}{|c|c|c|c|c|c|c|c|c|c|}
\hline item & nit & eq. & 1st & 3rd & 5 th & 7 th & 9 th & 11th & $13 \mathrm{th}$ \\
\hline \multirow{6}{*}{$\begin{array}{l}\text { sending } \\
\text { end } \\
\text { voltage }\end{array}$} & \multirow{3}{*}{ KV } & $T$ & 31.88 & 0.0231 & 0.0793 & 15.583 & 0.0100 & 0.0775 & 1.5614 \\
\hline & & - & 4.7153 & 0.0145 & 1.6609 & 1.4238 & 0.01 .15 & 0.1728 & 0.7471 \\
\hline & & 0 & 1.1382 & 0.8609 & 0.1718 & 0.0482 & 0.2794 & 0.3102 & 0.0252 \\
\hline & \multirow{3}{*}{$\% * *$} & + & 100.00 & 2.6800 & $4.770^{*}$ & 100.00 & $3.570^{*}$ & $44.85^{*}$ & 100.00 \\
\hline & & - & $2.030^{*}$ & $1.690^{*}$ & 100.00 & $9.140^{*}$ & $4.120^{*}$ & 100.00 & $47.85^{*}$ \\
\hline & & 0 & $0.490^{*}$ & 100.00 & $10.34^{*}$ & $0.310^{*}$ & 100.00 & $179.4^{*}$ & $1.610^{*}$ \\
\hline \multirow{6}{*}{$\begin{array}{l}\text { receiving } \\
\text { end } \\
\text { voltage }\end{array}$} & \multirow{3}{*}{ KV } & + & 266.28 & 0.4788 & 0.0942 & 17.349 & 0.1066 & 0.0957 & 1.6938 \\
\hline & & - & 5.3906 & 0.3010 & 1.9716 & 1.5859 & 0.1228 & 0.2133 & 0.8105 \\
\hline & & 0 & 1.3969 & 1.3325 & 0.1554 & 0.0891 & 0.2672 & 0.3839 & 0.0331 \\
\hline & \multirow{3}{*}{$\% * *$} & $t$ & 100.00 & $35.93^{*}$ & $4.780^{*}$ & 100.00 & $39.91^{*}$ & $44.85^{*}$ & 100.00 \\
\hline & & - & $2.020^{*}$ & $22.58^{*}$ & 100.00 & $9.140^{*}$ & $45.97^{*}$ & 100.00 & $47.85^{*}$ \\
\hline & & 0 & $0.520^{*}$ & 100.00 & $7.880^{*}$ & $0.510^{*}$ & 100.00 & $179.8^{*}$ & $1.950^{*}$ \\
\hline \multirow{6}{*}{$\begin{array}{l}\text { generator } \\
\text { current }\end{array}$} & \multirow{3}{*}{$\mathrm{KA}$} & + & 0.3294 & 0.0001 & 0.0002 & 0.0223 & 0.0000 & 0.0001 & 0.0012 \\
\hline & & - & 0.0055 & 0.0000 & 0.0033 & 0.0020 & 0.0000 & 0.0002 & 0.0006 \\
\hline & & 0 & 0.0013 & 0.0086 & 0.0010 & 0.0002 & 0.0009 & 0.0008 & 0.0001 \\
\hline & \multirow{3}{*}{$\% * *$} & $t$ & 100.00 & $0.890^{*}$ & $4.780^{*}$ & 100.00 & $1.190^{*}$ & $44.87^{*}$ & 100.00 \\
\hline & & - & $1.660^{*}$ & $0.570 *$ & 100.00 & $9.140^{*}$ & $1.370^{*}$ & 100.00 & $47.84^{*}$ \\
\hline & & 0 & $0.400^{*}$ & 100.00 & $31.14^{*}$ & $0.940^{*}$ & 100.00 & $539.8 *$ & $4.880^{*}$ \\
\hline
\end{tabular}

* - These components shall be zero if the system is balanced.

** - Normalized to the dominant sequence quantity of each harmonic.

These observations confirm the considerations in Chapter 1: the harmonics are more 
sensitive to unbalances. It therefore justifies the necessity of a multiphase approach to the harmonic load flow analysis of power systems.

\subsubsection{Numerical Convergence of the Solution}

The convergence behaviour of the MHLF technique is listed in Table 4.2 for both test cases. It shows the maximum change of the equivalent current $\mathbf{I}_{h-e q}$ between successive iterations. A rapid convergence is obtained. In both test cases, up to the 15 th order harmonic was computed in the MHLF solution.

Table 4.2: Convergence behaviour.

\begin{tabular}{|c|cc|}
\hline Iteration \# & Balanced & Unbalanced \\
\hline 1 & 0.01354 & 0.01420 \\
2 & 0.00143 & 0.00134 \\
3 & 0.00014 & 0.00014 \\
4 & 0.00003 & 0.00003 \\
\hline CPU(s) & 7.40 & 8.57 \\
\hline
\end{tabular}

\subsection{MHLF technique for EMTP Initialization}

Although the MHLF technique is developed as a tool for multiphase harmonic analysis, the technique is also applicable as an improved initialization procedure for EMTP transient simulations. For this application, two options of improved initialization can be provided. They enable the EMTP simulation to be initialized either with only the fundamental frequency load flow results or with the harmonic load flow results.

\subsubsection{General Considerations}

The traditional initialization procedure in the EMTP is simply the solution of nodevoltage matrix equations. It is therefore restricted to constant impedance loads and fixed 

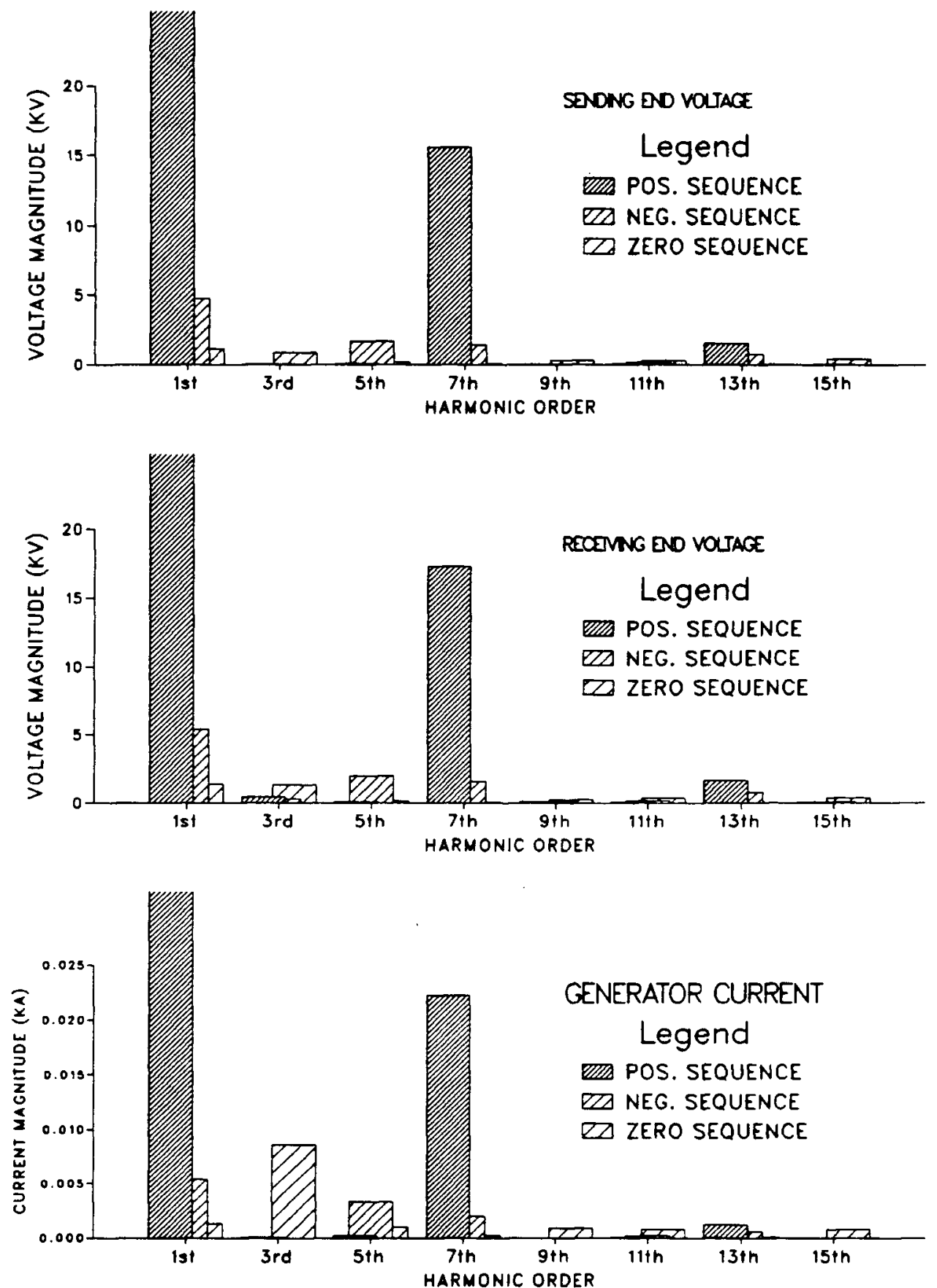

Figure 4.7: Analysis of harmonic unbalances. 
voltage or current sources. Although additional load flow constraints were included later [12], this function still has limited capabilities in the modelling of loads and machines. The convergence characteristics can also be unsatisfactory or poor. The inclusion of harmonic components in the EMTP initialization scheme was not considered until a recent work [14]. In the work of [14], the harmonics generated from nonlinear inductors are taken into account. However, the load flow constraints are not modelled in the process.

The MHLF initialization is a comprehensive improvement over the existing methods, and also is a straightforward process. With the MHLF (harmonic) load flow results, the initial and historic values of network voltages and currents can readily be computed. These values in conjunction with the network components are then used to construct the the EMTP required transient models.

\subsubsection{Load Models in the EMTP Transient Simulation}

In spite of the mathematical simplicity, special attention must be paid to load modelling in the transient simulations. Due to the lack of knowledge on transient behaviours of most loads, general transient load models are still not available. In one of the recent studies, the loads are modelled as $R, L, C$ subnetworks whose parameters are determined by a system identification technique [29]. Since such data have not yet been widely available, and as a compromise, the harmonic load models recommended in reference [22] and used in the MHLF program described in Chapter 3 of this work are also adopted to represent the load behaviour in EMTP simulations. With this condition, the constant PQ loads (type-2 and -3) are modelled as a RL equivalent subnetworks as has been done in Sections 2.2 and 3.6. Rotating motor loads (type-4) are modelled in the same way as machines as shown in Section 3.6. 


\subsection{Summary}

Based on the multiphase network solution program of Chapter 3 and the Norton equivalent model of nonlinear elements, the single-phase harmonic method of Chapter 2 has been extended into a multiphase harmonic load flow technique. The general solution process of the technique has been outlined in this chapter. As a tool of multiphase harmonic and load flow analysis, this technique possesses some very useful characteristics.

The most important characteristic is the flexibility and extendibility of the technique. The harmonics from any nonlinear element can easily be analyzed. This is achieved by first developing a frequency-domain model of the element, and then interfacing the model with the main MHLF iteration process. The MHLF program has been structured in such a way that the user can analyze any nonlinear element by only supplying a subroutine. This user-supplied subroutine computes the Norton equivalent circuit of the nonlinear element with the MHLF program giving the network current and voltage conditions. One of the best approaches to derive the equivalent circuits may be the time-domain technique used for the nonlinear inductor model development presented in this chapter. However, the MHLF iteration process allows other approaches as well.

The second important characteristic is that the MHLF technique provides a wide range of application options. They include:

1. Multiple source frequency scan solutions.

2. Multiple frequency solutions [1]. In this option, the nonlinear elements are represented as pre-determined harmonic current sources.

3. Harmonic load flow solution with iteration $=1$. In this option, the nonlinear elements are modelled as Norton equivalent circuits computed from the fundamental 
frequency load flow solutions only ${ }^{1}$.

4. Comprehensive harmonic load flow solutions.

All the options are available for either balanced or unbalanced analysis, and for either single-phase or multiphase solutions.

The MHLF technique is also computationally efficient. First of all, the initialization of the iterative process that uses only the fundamental frequency components is simple. Because the harmonics in power networks are relative small compared with the fundamental frequency component, starting the iteration with fundamental frequency components has been found quite reliable. Secondly, because the Norton equivalent circuits are harmonically-decoupled, the network solutions are performed one frequency at the time. Thus the size of the Jacobian matrix is reduced considerably and the computational burden is only linearly proportional to the number of total harmonics. Thirdly, the technique has exhibited good convergence behaviour.

For the multiphase fundamental frequency load flow analysis, the program provides a very powerful tool to analyze network components with unusual load flow constraints, such as HVDC links. This can be achieved by also developing a Norton equivalent circuit of that component, and interfacing it with the MHLF process. As mentioned before, the program has been structured to facilitate such implementations.

The MHLF technique has therefore satisfied the general requirements set in Chapter 1. In view of the various nonlinearities that exist in power networks, the technique is further improved and enhanced as shown in the next chapters.

\footnotetext{
${ }^{1}$ This option is also useful to identify convergence difficulties, see Chapter 7 .
} 


\section{Chapter 5}

\section{Modelling of Synchronous Machines as Harmonic Sources}

Besides nonlinear inductors, another major source of harmonics in power systems is the synchronous machines. It has been known for years that synchronous machines may generate harmonics when operated under unbalanced conditions. Presently available machine models are not sufficient for the investigation of such harmonic effects. A new machine model for this harmonic analysis was thus developed. It was then integrated into the MHLF program by including load flow constraints.

\subsection{Harmonic Generation of Synchronous Machines}

There are many ways that a synchronous machine can produce harmonic distortions in a power network. Among the various causes, the most significant ones are the frequency conversion process and the inherent saturation effects. Both effects take place when the machine is operated under unbalanced conditions.

Under unbalanced conditions, a machine may experience a negative sequence current in its armature. This current, depending on the saliency of the rotor, may induce a second order harmonic in the rotor. The rotor harmonic in turn may induce the third order harmonic back into the armature, and so on. This is the process of frequency conversion which takes place for higher order unbalanced harmonics as well. The harmonic effects due to frequency conversion are two fold. First, the process causes a machine to generate harmonics. Second, it complicates the reaction of a machine to the harmonics produced 
from other sources.

The saturation of the excitation circuit is another cause of harmonic distortions. It interacts with the frequency conversion process, and therefore, adds extra complications to the problem.

Investigations on the machine harmonic behaviour have been carried out recently $[36,37]$. These works provide useful insight on the mechanism of frequency conversion. a harmonically-coupled admittance matrix model that includes frequency conversion effect has been developed in reference [37]. Although these studies are limited to machine analysis assuming the load flow results are available, they have established the basis for further development.

For the purpose of multiphase harmonic load flow analysis, such as the MHLF program, the machine harmonic problem must be considered in conjunction with the network load flow conditions. Moreover, the load flow control characteristics of the machines must be taken into consideration. Therefore, the problem of synchronous machine harmonic analysis becomes the study of a three-phase nonlinear network with control characteristics.

\subsection{The MHLF Iterative Scheme}

In the original MHLF analysis described in previous chapters, a synchronous machine was modelled as a set of positive sequence voltage sources behind a known admittance matrix, as shown in Figure 3.1(b). The voltage sources are unknown and must be adjusted by the load flow solution process to satisfy the machine constraints.

Although this simple model is useful to some extent, it is not valid for frequency conversion and saturation analysis. Thus, a machine model which includes the effects 
of frequency conversion and saturation is developed (see Figure 5.1). This model is also directly applicable to harmonic load flow analysis.

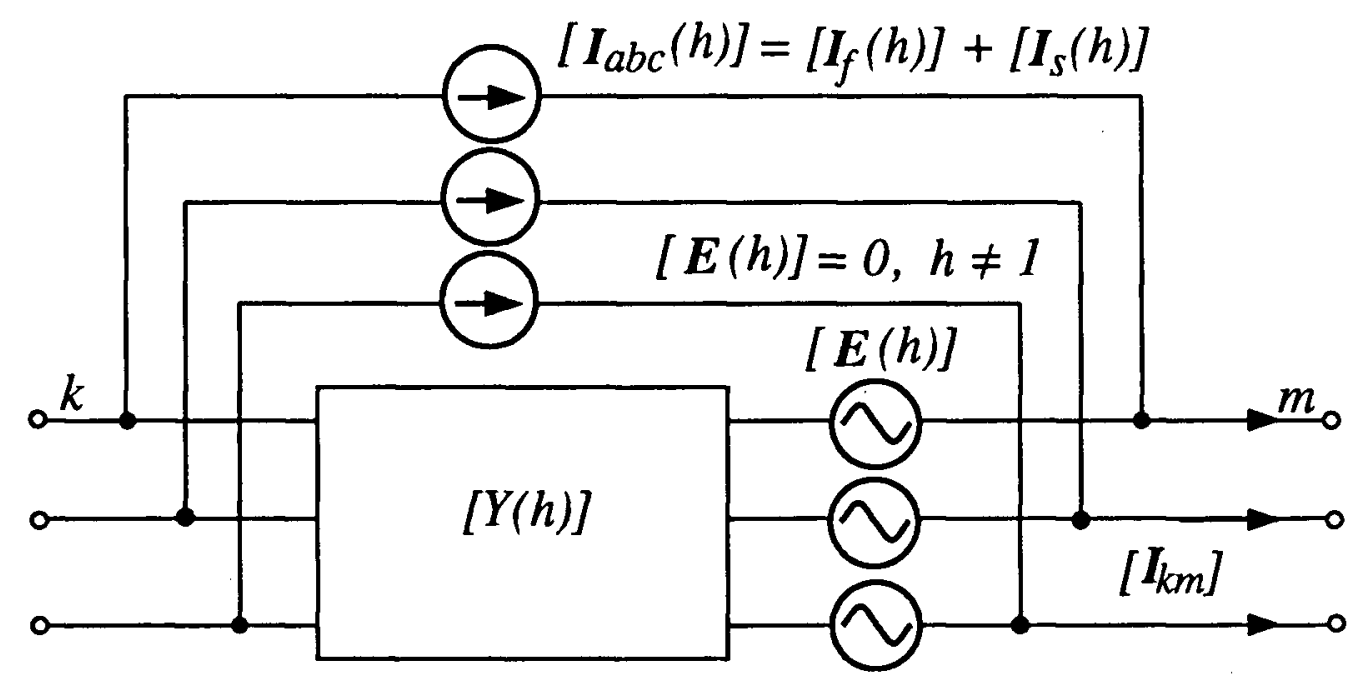

Figure 5.1: A synchronous machine model with nonlinear effects included.

As shown in the figure, the model consists of the machine admittance matrix at harmonic order $h,[Y(h)]$, the positive sequence voltage source $[\mathbf{E}(h)]$, and the harmonic current source $\left[\mathbf{I}_{a b c}(h)\right]$. The nonlinear effects are included in $\left[\mathbf{I}_{a b c}(h)\right]$. This harmonic current source which is the sum of harmonic components from frequency conversion $\left(\left[\mathbf{I}_{f}(h)\right]\right)$ and saturation $\left(\left[\mathbf{I}_{s}(h)\right]\right)$ is known beforehand. It can be seen that the model becomes the original form (Figure $3.1(b)$ ) if $\left[\mathbf{I}_{a b c}(h)\right]$ is zero. Since $\left[\mathbf{I}_{a b c}(h)\right]$ is a simple known current source, the model is linear and harmonically-decoupled. Interfacing the model with the MHLF solution process would calculate the harmonic contributions by the synchronous machine.

As mentioned before in Chapter 3, a machine is subject to the SK, PV and PQ load flow constraints. To include these constraints, the equation representing the machine 
model in the multiphase load flow equation set (Eqs. (3.26)-(3.32)) is modified. Namely, Eq. (3.31) is replaced with the newly developed machine model of Figure 5.1. Since the additional harmonic current component $\left[\mathbf{I}_{a b c}(h)\right]$ in the extended model is a known current source, there is no extra complication for the Newton-Raphson solution process.

Similarly to the case of nonlinear inductors, the parallel current source $\left[\mathbf{I}_{a b c}(h)\right]$ is only known approximately. It is estimated from the load flow results prior to the improved machine models being applied. An iterative process is therefore needed for accurate solution. This is the harmonic iteration process built into the MHLF technique. The iteration starts with the assumption that $\left[\mathbf{I}_{a b c}(h)\right]$ is equal to zero. It converges when the change of $\left[\mathbf{I}_{a b c}(h)\right]$ between two successive iterations is within specified criteria.

The remaining problem is the development of the new machine model shown in Figure 5.1. This is explained in the following sections.

\subsection{Machine Model with Frequency Conversion}

Each machine has different design characteristics in general. To develop a general purpose machine model for harmonic analysis, a number of idealized characteristics need to be assumed. For the purpose of initializing the machine transient simulations, the original assumptions in the EMTP are retained [12]. As a result, following Park transformation can be used to transform the machine quantities from abc-coordinates into the dqocoordinates:

$$
\left[\begin{array}{lll}
v_{d} & v_{q} & v_{o}
\end{array}\right]^{T}=[P]^{-1}\left[\begin{array}{lll}
v_{a} & v_{b} & v_{c}
\end{array}\right]^{T}
$$

where

$$
[P]^{-1}=\sqrt{2 / 3}\left[\begin{array}{lll}
\cos (\omega t+\delta) & \cos (\omega t+\delta-2 \pi / 3) & \cos (\omega t+\delta+2 \pi / 3) \\
\sin (\omega t+\delta) & \sin (\omega t+\delta-2 \pi / 3) & \sin (\omega t+\delta+2 \pi / 3) \\
\sqrt{1 / 2} & \sqrt{1 / 2} & \sqrt{1 / 2}
\end{array}\right]
$$


and $\delta$ is the angle between $\mathrm{d}$-axis and the real axis for the network phasor frame. Defining

$$
[D]=\left[\begin{array}{lll}
1 & e^{-j 2 \pi / 3} & e^{-j 4 \pi / 3} \\
-j & -j e^{-j 2 \pi / 3} & -j e^{-j 4 \pi / 3} \\
0 & 0 & 0
\end{array}\right] e^{j \delta} / \sqrt{6}, \text { and }\left[D_{0}\right]=\left[\begin{array}{lll}
0 & 0 & 0 \\
0 & 0 & 0 \\
\sqrt{1 / 3} & \sqrt{1 / 3} & \sqrt{1 / 3}
\end{array}\right]
$$

the matrix $[P]^{-1}$ is then simplified as:

$$
[P]^{-1}=[D] e^{j \omega t}+[D]^{C} e^{-j \omega t}+\left[D_{0}\right] .
$$

Since the Park transformation is an orthogonal transformation, it follows that

$$
[P]=\left([P]^{-1}\right)^{T}=[D]^{T} e^{j \omega t}+[D]^{H} e^{-j \omega t}+\left[D_{o}\right]^{T} .
$$

where superscripts $\mathrm{T}, \mathrm{C}$, and $\mathrm{H}$ represent transpose, conjugate, and conjugate transpose respectively. With the park transformation, the machine model including the effects of both frequency conversion and saturation can be derived. For clarity, the model with only frequency conversion is developed at first. It produces the machine admittance matrix $[Y(h)]$ and the equivalent harmonic current source $\left[\mathrm{I}_{f}(h)\right]$ resulted from the frequency conversion process.

\subsubsection{Harmonic Machine Model in DQO-coordinates}

With the usual generator conventions, a synchronous machine can be described in dqoor Park-coordinates as:

$$
\begin{gathered}
{\left[v_{p a r k}\right]=-[R]\left[i_{p a r k}\right]-\frac{d}{d t}\left[\lambda_{p a r k}\right]+[F]\left[\lambda_{p a r k}\right]} \\
{\left[\lambda_{p a r k}\right]=[L]\left[i_{p a r k}\right]}
\end{gathered}
$$

where

$$
\left[v_{p a r k}\right]=\left[\begin{array}{lllllll}
v_{d} & v_{q} & v_{0} & v_{f} & v_{g} & v_{D} & v_{Q}
\end{array}\right]^{T}
$$




$$
\begin{aligned}
& {\left[i_{p a r k}\right]=\left[\begin{array}{lllllll}
i_{d} & i_{q} & i_{0} & i_{f} & i_{g} & i_{D} & i_{Q}
\end{array}\right]^{T}} \\
& {[R]=\left[\begin{array}{lllllll}
r_{a} & r_{a} & r_{a} & r_{f} & r_{g} & r_{D} & r_{Q}
\end{array}\right]^{T}} \\
& {[F]=\left[\begin{array}{lllllll}
0 & -\boldsymbol{\omega} & 0 & 0 & 0 & 0 & 0 \\
\omega & 0 & 0 & 0 & 0 & 0 & 0 \\
0 & 0 & 0 & 0 & 0 & 0 & 0 \\
0 & 0 & 0 & 0 & 0 & 0 & 0 \\
0 & 0 & 0 & 0 & 0 & 0 & 0 \\
0 & 0 & 0 & 0 & 0 & 0 & 0 \\
0 & 0 & 0 & 0 & 0 & 0 & 0
\end{array}\right]} \\
& {[L]=\left[\begin{array}{lllllll}
L_{d} & 0 & 0 & M_{d f} & 0 & M_{d D} & 0 \\
0 & L_{q} & 0 & 0 & M_{q g} & 0 & M_{g Q} \\
0 & 0 & L_{o} & 0 & 0 & 0 & 0 \\
M_{d f} & 0 & 0 & L_{f f} & 0 & M_{f D} & 0 \\
0 & M_{q g} & 0 & 0 & L_{g g} & 0 & M_{g Q} \\
M_{d D} & 0 & 0 & M_{f D} & 0 & L_{D D} & 0 \\
0 & M_{q Q} & 0 & 0 & M_{g Q} & 0 & L_{Q Q}
\end{array}\right]}
\end{aligned}
$$

The subscripts $f$ and $D$ represent the field and damper windings in $d$-axis; $g$ and $Q$ represent two damper windings in q-axis, respectively. Substituting Eq. (5.2) into Eq. (5.1), and defining the differential operator $p=\frac{d}{d t}$, we have

$$
\begin{aligned}
{\left[v_{\text {park }}\right] } & =-[R]\left[i_{\text {park }}\right]-p\left([L]\left[i_{\text {park }}\right]\right)+[F][L]\left[i_{\text {park }}\right] \\
& =(-[R]-p[L]+[F][L])\left[i_{\text {park }}\right] \\
& =[Z(p)]\left[i_{\text {park }}\right]
\end{aligned}
$$

where

$$
[Z(p)]=-[R]-p[L]+[F][L]
$$


is the operational impedance matrix. Since the rotor angular speed $\omega$ is constant in the steady-state, Eq. (5.3) represents a linear time invariant system. Hence it can be analyzed in the frequency-domain at each harmonic frequency $h \omega$ as:

$$
\left[\mathbf{V}_{\text {park }}(h)\right]=[Z(h)]\left[\mathbf{I}_{\text {park }}(h)\right],
$$

where the bold-faced symbols indicate phasor quantities.

In the cases of harmonic components $h \neq 0$, the $g, D$ and $Q$ windings are shortcircuited. If we assume that the field winding is energized with an undistorted DC voltage source, the field winding becomes short-circuited as well. Eq. (5.4) can therefore be rewritten as

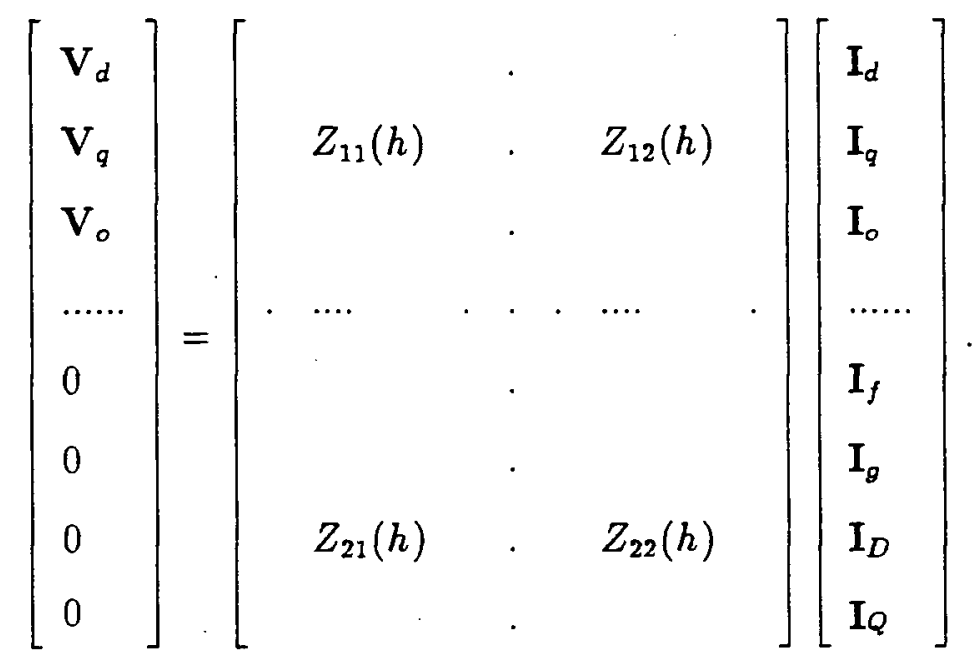

Solving for dqo-currents as a function of dqo-voltages yields

$$
\begin{aligned}
{\left[\mathbf{I}_{d q o}(h)\right] } & =\left[Z_{11}(h)-Z_{12}(h) Z_{22}^{-1}(h) Z_{21}(h)\right]^{-1}\left[\mathbf{V}_{d q o}(h)\right] \\
& =\left[Y_{d q o}(h)\right]\left[\mathbf{V}_{d q o}(h)\right],
\end{aligned}
$$

where

$$
\begin{aligned}
{\left[Y_{d q o}(h)\right] } & =\left[Z_{11}(h)-Z_{12}(h) Z_{22}^{-1}(h) Z_{21}(h)\right]^{-1} \\
{\left[\mathbf{I}_{d q o}(h)\right] } & =\left[\begin{array}{lll}
\mathbf{I}_{d} & \mathbf{I}_{q} & \mathbf{I}_{o}
\end{array}\right]^{T} \\
{\left[\mathbf{V}_{d q o}(h)\right] } & =\left[\begin{array}{lll}
\mathbf{V}_{d} & \mathbf{V}_{q} & \mathbf{V}_{o}
\end{array}\right]^{T}
\end{aligned}
$$


Eq. (5.5) defines the harmonic machine model in dqo-coordinates. As expected, this model consists of a simple admittance matrix coupling dqo-components.

In the case of harmonic order $h=0$, the voltage of f-winding is no longer zero. Same operations can still be performed however. The results are similar to Eq. (5.5) except that there is an equivalent $\mathrm{DC}$ voltage source in series with the $\left[Y_{\text {dqo }}\right]$ matrix. This voltage source is a function of the DC excitation voltage $v_{f}$.

\subsubsection{Harmonic Machine Model in ABC-coordinates}

To relate the machine model of Eq. (5.5) in dqo-coordinate to the rest of the network which is described in abc-coordinates, the respective dqo-components can be converted into phase quantities by Park transformation. Assume that the machine voltage $\left[v_{a b c}(t)\right]$ includes the harmonic component of order $h$ :

$$
\left[v_{a b c}(t)\right]=\operatorname{Real}\left\{\sqrt{2}\left[\mathbf{V}_{a b c}(h)\right] e^{j h \omega t}\right\} .
$$

The corresponding dqo-voltage is then computed by Park transformation:

$$
\begin{aligned}
{\left[v_{d q o}(t)\right] } & =[P]^{-1}\left[v_{a b c}(t)\right] \\
& =\operatorname{Real}\left\{[P]^{-1} \sqrt{2}\left[\mathbf{V}_{a b c}(h)\right] e^{j h \omega t}\right\} \\
& =\sqrt{2} \operatorname{Real}\left\{[D]\left[\mathbf{V}_{a b c}(h)\right] e^{j(h+1) \omega t}+[D]^{C}\left[\mathbf{V}_{a b c}(h)\right] e^{j(h-1) \omega t}+\left[D_{o}\right]\left[\mathbf{V}_{a b c}(h)\right] e^{j h \omega t}\right\}
\end{aligned}
$$

Note that the one harmonic abc-voltage has introduced three harmonics in the dqocoordinates. By this time, the machine dqo-model can be applied. The resultant dqocurrent is obtained by using Eq. (5.5):

$$
\begin{array}{rlrl}
{\left[i_{d q o}(t)\right]=\sqrt{2} \operatorname{Real}\{} & & {\left[Y_{d q o}(h+1)\right][D]\left[\mathbf{V}_{a b c}(h)\right] e^{j(h+1) \omega t}} \\
+ & {\left[Y_{d q o}(h-1)\right][D]^{C}\left[\mathbf{V}_{a b c}(h)\right] e^{j(h-1) \omega t}} \\
+ & \left.\left[Y_{d q o}(h)\right]\left[D_{o}\right]\left[\mathbf{V}_{a b c}(h)\right] e^{j h \omega t}\right\}
\end{array}
$$


Transforming this current back into abc-coordinates through $[\mathrm{P}]$ matrix, and using the condition

$$
[D]^{T}\left[D_{\circ}\right]=0
$$

we have

$$
\begin{aligned}
{\left[i_{a b c}(t)\right]=} & {[P]\left[i_{d q o}(t)\right]=\sqrt{2} R e a l } \\
\{ & {\left[D_{o}^{T} Y_{d q o}(h) D_{\circ}+D^{T} Y_{d q o}(h-1) D^{C}+D^{H} Y_{d q o}(h+1) D\right]\left[\mathbf{V}_{a b c}(h)\right] e^{j h \omega t} } \\
+ & {\left[D^{H} Y_{d q o}(h-1) D^{C}\right]\left[\mathbf{V}_{a b c}(h)\right] e^{j(h-2) \omega t} } \\
+ & {\left.\left[D^{T} Y_{d q o}(h+1) D\right]\left[\mathbf{V}_{a b c}(h)\right] e^{j(h+2) \omega t}\right\} }
\end{aligned}
$$

It can be seen from the above equation that the harmonic voltage of order $h,\left[\mathbf{V}_{a b c}(h)\right]$, could generate the harmonic currents of order $h-2, h$, and $h+2$ in abc-coordinates. This is the result of frequency conversion. The conversion process is also shown in more detail in Figure 5.2. As shown in the figure, the negative sequence voltage $[D]\left[\mathbf{V}_{a b c}(h)\right]$ generates harmonic currents of order $h+2$ and $h$; while the positive sequence voltage $[D]^{C}\left[\mathrm{~V}_{a b c}(h)\right]$ generates harmonic currents of order $h-2$ and $h$. The zero sequence voltage $\left[D_{o}\right]\left[\mathbf{V}_{a b c}(h)\right]$ generates only the harmonic current of the same order. All these observations are consistent with the rotating flux explanation [38].

Eq. (5.6) also indicates that there are three harmonic voltages which can generate harmonic currents of same order. Assuming that the machine voltage $\left[v_{a b c}(t)\right]$ consists of all harmonic components, the harmonic terminal voltages that result in the currents of same harmonic order $h$ can be grouped and described in a phasor form as:

$$
\begin{aligned}
{\left[\mathrm{I}_{a b c}(h)\right] } & =\left[D^{T} Y_{d q o}(h-1) D^{C}+D^{H} Y_{d q o}(h+1) D+D_{o}^{T} Y_{d q o}(h) D_{o}\right]\left[\mathbf{V}_{a b c}(h)\right] \\
& +\left[D^{H} Y_{d q o}(h+1) D^{C}\right]\left[\mathbf{V}_{a b c}(h+2)\right] \\
& +\left[D^{T} Y_{d q o}(h-1) D\right]\left[\mathbf{V}_{a b c}(h-2)\right]
\end{aligned}
$$




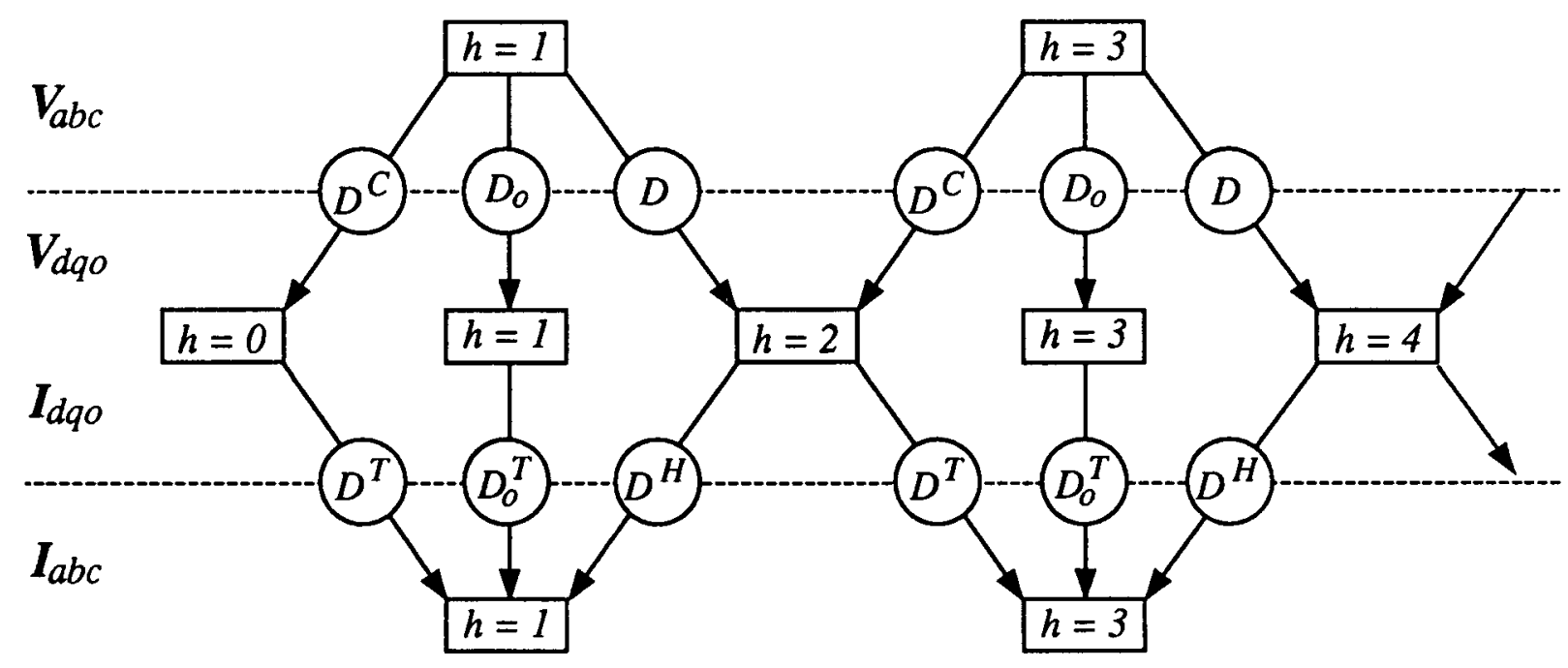

Figure 5.2: Process of frequency conversion.

Denote

$$
\begin{gathered}
{[Y(h)]=\left[D^{T} Y_{d q o}(h-1) D^{C}+D^{H} Y_{d q o}(h+1) D+D_{o}^{T} Y_{d q o}(h) D_{o}\right]} \\
{\left[\mathbf{I}_{f}(h)\right]=\left[D^{H} Y_{d q o}(h+1) D^{C}\right]\left[\mathbf{V}_{a b c}(h+2)\right]+\left[D^{T} Y_{d q o}(h-1) D\right]\left[\mathbf{V}_{a b c}(h-2)\right]}
\end{gathered}
$$

Eq. (5.7) can be rewritten as

$$
\left[\mathbf{I}_{a b c}(h)\right]=[Y(h)]\left[\mathbf{V}_{a b c}(h)\right]+\left[\mathbf{I}_{f}(h)\right]
$$

This is the equation that defines the machine model shown in Figure 5.1. Correspondingly, the admittance matrix $[Y(h)]$ represents the effects of the harmonic voltage of the same order as the current. The harmonic current $\left[\mathbf{I}_{f}(h)\right]$ describes the harmonic coupling of frequency conversion effects. This current is generated from the conversion of the machine voltages of different harmonic orders.

Following a similar process, the equivalent DC voltage source in the dqo-machine model of harmonic order $h=0$ becomes a set of positive sequence fundamental frequency 
voltage sources in abc-coordinates. As explained earlier, their values are determined in conjunction with the load flow constraints.

\subsubsection{Summary}

It can be seen that only simple matrix operations are needed to compute the admittance matrix $[Y(h)]$ and the equivalent current source $\left[\mathbf{I}_{f}(h)\right]$. This process is summarized in the following:

1. Compute $[Y(h)]$ according to Eq. (5.8) whenever the frequency scan process needs the machine admittance matrix to be added to the network admittance matrix.

2. Compute $\left[\mathbf{I}_{f}(h)\right]$ according to Eq. (5.9), where $\left[\mathbf{V}_{a b c}(h)\right]$ is the machine voltage obtained from the load flow solutions of last iteration.

It needs to be noted that $[Y(h)]$ matrix is unsymmetric in general, since the responses of a machine to the positive and negative sequence harmonic voltages are different. However, in the case of fundamental frequency, the admittance matrix can be made symmetric by setting the positive sequence admittance equal to the negative sequence admittance, as shown in Section 3.2.3.

\subsection{Inclusion of Saturation Effects}

It is well-known that the saturation of air-gap flux in a synchronous machine has most significant effects on its operation [12]. Other saturation factors either are negligible from network study point of view or have little contribution for harmonic load flows [38]. Therefore, only the air-gap flux saturation is studied in this work. 


\subsubsection{Saturation Effects in DQO-coordinates}

It is shown in Appendix A that the effects of air-gap flux saturation can be represented as a current source in parallel with the dqo-machine model developed in previous section (Eq. (5.5)):

$$
\left[\mathbf{I}_{d q o}(h)\right]=\left[Y_{d q o}(h)\right]\left[\mathbf{V}_{d q o}(h)\right]+\left[\mathbf{I}_{d q o-o}(h)\right],
$$

where $\left[\mathbf{I}_{d q o-s}(h)\right]$ is a known current vector representing the effects of saturation. It is obtained with given machine voltage conditions through a subiterative process described in Appendix A.

\subsubsection{Saturation Effects in ABC-coordinates}

With this newly developed machine model in dqo-coordinate, the Park transformation can again be performed to obtain the machine model in abc-coordinates. Since the first part of Eq. (5.11) has been processed in the previous section, only the second part which is the current source $\left[\mathbf{I}_{d q o-s}(h)\right]$ needs to be discussed. With the transformation matrix $[\mathrm{P}]$, the dqo harmonic current of order $h$ appears in abc-coordinates as:

$$
\begin{aligned}
{\left[i_{a b c-s}(t)\right] } & =\operatorname{Real}\left\{[P] \sqrt{2}\left[\mathbf{I}_{d q o-s}(h)\right] e^{j h \omega t}\right\} \\
& =\operatorname{Real}\left\{\sqrt{2}\left[D^{T} e^{j(h+1) \omega t}+D^{H} e^{j(h-1) \omega t}+D_{\circ} e^{j h \omega t}\right]\left[\mathbf{I}_{d q o-s}(h)\right]\right\} .
\end{aligned}
$$

It is seen that $\left[\mathbf{I}_{d q 0-s}(h)\right]$ also introduces three separate harmonic components of order $h-1, h$ and $h+1$ into the abc-coordinates. Assume that $\left[\mathbf{I}_{d q o-s}(h)\right]$ consists of harmonics of all orders, the dqo-harmonics that result in harmonic currents with same order in the abc-coordinates can be grouped together in a phasor form as:

$$
\left[\mathbf{I}_{\boldsymbol{s}}(h)\right]=[D]^{T}\left[\mathbf{I}_{d q o-s}(h-1)\right]+[D]^{H}\left[\mathbf{I}_{d q o-s}(h+1)\right]+\left[D_{\mathrm{o}}\right]\left[\mathbf{I}_{d q o-s}(h)\right] \text {. }
$$

This is the current that is needed to represent the effects of saturation in abc-coordinates. The entire machine model with both the frequency conversion and saturation effects 
included can then be described by

$$
\left[\mathbf{I}_{a b c}(h)\right]=[Y(h)]\left[\mathbf{V}_{a b c}(h)\right]+\left[\mathbf{I}_{f}(h)\right]+\left[\mathbf{I}_{\boldsymbol{s}}(h)\right] .
$$

The above equation defines the final machine model used for the harmonic load flow studies. The process of computing $\left[\mathbf{I}_{\delta}(h)\right]$ is summarized in the following:

1. Compute the saturation current in dqo-coordinates, $\left[\mathbf{I}_{d q o-s}(h)\right]$, by a subiteration process described in Appendix A.

2. Compute $\left[\mathbf{I}_{\boldsymbol{s}}(h)\right]$ according to Eq. (5.12) for all harmonics of interest.

\subsection{Case Studies}

The significance of synchronous machine frequency conversion and saturation in producing harmonics is demonstrated by the following case studies. The performance of the MHLF program is also investigated with the examples.

\subsubsection{Unbalanced Harmonic Solutions}

(1) Third harmonic current induced by unbalance operation.

The effects of frequency conversion are first evaluated by inspecting the induced third harmonic current $I_{f}(3)$ when the machines are operated under different unbalanced conditions. Five salient pole machines were selected for the study. The machine data obtained from references [36], [37], and [38] have been listed in Appendix E. Table 5.1 lists the results of such studies. In the table, the percentage third harmonic current $I_{f}(3)$ is computed for different degrees of fundamental frequency voltage and current unbalance. The percentage harmonic is defined as the ratio of that harmonic to the fundamental frequency component. The unbalance is defined as the ratio of the negative sequence quantity to the positive sequence quantity. 
As shown in the table, the third harmonic current injection into the network is proportional to the degree of either voltage or current unbalance. It is noted in some cases that the harmonic current injections could reach $3 \%$ when the machines experience $10 \%$ negative sequence current. This observation suggests that the unbalance induced third harmonic current is not negligible even under the normal operating conditions of the machines [39].

Table 5.1: Third harmonic current caused by fundamental frequency voltage and current unbalance for five test machines.

\begin{tabular}{|c|c|c|c|c|c|}
\hline \multirow{2}{*}{$\begin{array}{c}\text { Machine } \\
\#\end{array}$} & \multicolumn{5}{|c|}{ fundamental frequency voltage unbalance (\%) } \\
\hline & 2.00 & 4.00 & 6.00 & 8.00 & 10.00 \\
\hline & \multicolumn{5}{|c|}{ Generated third harmonic current (\%) } \\
\hline 1 & 1.19 & 2.39 & 3.58 & 4.77 & 5.97 \\
\hline 2 & 4.70 & 9.40 & 14.11 & 18.81 & 23.51 \\
\hline 3 & 0.91 & 1.82 & 2.74 & 3.65 & 4.56 \\
\hline 4 & 0.30 & 0.60 & 0.90 & 1.20 & 1.50 \\
\hline 5 & 2.25 & 4.51 & 6.76 & 9.02 & 11.27 \\
\hline Machine & \multicolumn{5}{|c|}{ fundamental frequency current unbalance (\%) } \\
\hline$\#$ & 2.00 & 4.00 & 6.00 & 8.00 & 10.00 \\
\hline & \multicolumn{5}{|c|}{ Generated third harmonic current (\%) } \\
\hline 1 & 0.47 & 0.94 & 1.41 & 1.88 & 2.35 \\
\hline 2 & 1.09 & 2.19 & 3.28 & 4.38 & 5.47 \\
\hline 3 & 0.29 & 0.58 & 0.87 & 1.16 & 1.45 \\
\hline 4 & 0.05 & 0.11 & 0.16 & 0.21 & 0.27 \\
\hline 5 & 0.59 & 1.17 & 1.76 & 2.34 & 2.93 \\
\hline
\end{tabular}

(2) Harmonic profile study.

Machines 1 and 2 are further selected for harmonic profile studies. Generally, the voltage and current harmonics of a machine are dependent on the external network conditions. To simplify the interpretation of the results, a straightforward operating condition is considered. In this case, a machine is operated in grounded $\mathrm{Y}$ connection with unbalanced R, L, and C loads as shown in Figure 5.3. The loads are determined so that 


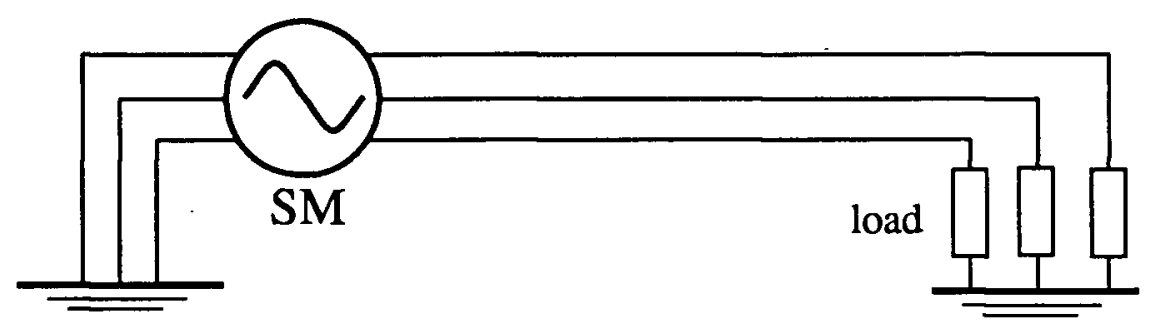

Figure 5.3: One machine test system.

the machine current unbalance in fundamental frequency is around its general operating condition (10\%) [39]. The results are shown in Table 5.2 for the voltage harmonics and the frequency conversion induced equivalent current source $\mathbf{I}_{f}(h)$.

Significant harmonics are observed in these cases when the machines are operated in an acceptable range of unbalance. The effects of these harmonics would easily be magnified if the voltage resonance takes place, as illustrated in reference [37]. Note that the harmonic magnitudes reduce quickly in higher orders, which is consistent with the observation made in reference [36]. From these results, it may be concluded that only 3rd and 5th harmonics need to be included in the machine harmonic studies.

(3) Effects of $\Delta$ connection.

It has been a general practice to connect generators or the low side of their step-up transformer in $\Delta$ form for the cancellation of external third harmonics. The possibility of preventing the machine produced 3rd harmonic penetrating to the external network is investigated in this work. The results are shown in Table 5.3 for the simple operating condition of machine 1 (Figure 5.3). Comparison between the results from grounded $Y$ and $\Delta$ connections reveals that the harmonic cancellation does not work. It is found by further studies that the third harmonic generated by frequency conversion is in negative sequence instead of in the zero sequence. Thus it can not be canceled by $\Delta$ connection 
Table 5.2: Harmonic profiles of two synchronous machines.

\begin{tabular}{|c|c|c|c|c|c|c|c|c|}
\hline \multirow{2}{*}{ Machine } & \multirow{2}{*}{ Item } & \multicolumn{2}{|c|}{$\mathrm{h}=1$} & \multicolumn{2}{|c|}{$h=3$} & \multicolumn{2}{|c|}{$\mathrm{h}=5$} & \multirow{2}{*}{$\begin{array}{c}\mathrm{h}=7 \\
\text { Mag. }\end{array}$} \\
\hline & & Mag. & Angle & Mag. & Angle & Mag. & Angle & \\
\hline \multirow[t]{2}{*}{$\overline{\text { No.1 }}$} & $\overline{\mathrm{V}_{a}}$ & 1.103 & 1.19 & 0.0148 & $\overline{88.34}$ & 0.0002 & 148.48 & 0.0000 \\
\hline & $V_{b}$ & 1.025 & -121.51 & 0.0131 & -36.50 & 0.0002 & 23.80 & 0.0000 \\
\hline \multirow{4}{*}{$\begin{array}{l}\mathrm{NP}= \\
9.35 \%\end{array}$} & $V_{c}$ & 1.022 & 120.24 & 0.0131 & -151.57 & 0.0002 & -90.28 & 0.0000 \\
\hline & $\mathbf{I}_{f-a}$ & 0.027 & 167.30 & 0.0225 & -152.50 & 0.0002 & -99.54 & 0.0000 \\
\hline & $I_{f-b}$ & 0.027 & -72.70 & 0.0225 & 87.45 & 0.0002 & 140.46 & 0.0000 \\
\hline & $\mathbf{I}_{f-c}$ & 0.027 & 47.30 & 0.0225 & -32.44 & 0.0002 & 20.46 & 0.0000 \\
\hline \multirow[t]{2}{*}{$\overline{\text { No.2 }}$} & $\overline{\overline{V_{a}}}$ & 1.080 & $\overline{1.31}$ & 0.0345 & $\overline{92.16}$ & $\overline{0.0011}$ & 157.27 & 0.0000 \\
\hline & $\mathrm{V}_{b}$ & 1.041 & -121.69 & 0.0313 & -33.05 & 0.0009 & 32.45 & 0.0000 \\
\hline \multirow{4}{*}{$\begin{array}{c}N P= \\
12.41 \%\end{array}$} & $v_{c}$ & 1.030 & 120.33 & 0.0315 & -148.27 & 0.0010 & -82.75 & 0.0000 \\
\hline & $I_{f-a}$ & 0.025 & 155.27 & 0.0704 & -151.39 & 0.0016 & -94.80 & 0.0000 \\
\hline & $I_{f-b}$ & 0.025 & -84.73 & 0.0696 & 88.30 & 0.0016 & 145.20 & 0.0000 \\
\hline & $I_{f-c}$ & 0.025 & 35.27 & 0.0696 & -30.97 & 0.0016 & 25.20 & 0.0000 \\
\hline
\end{tabular}

NP - ratio of the negative to positive sequence current at fundamental frequency.

that is only effective for zero sequence components.

Table 5.3: Third harmonic voltages and currents with Y-g and $\Delta$ connection.

\begin{tabular}{|c|c||cc|cc|cc|}
\hline \multicolumn{2}{|c|}{ Item } & \multicolumn{2}{c|}{ Phase-A } & \multicolumn{2}{c|}{ Phase-B } & \multicolumn{2}{c|}{ Phase-C } \\
\cline { 3 - 8 } \multicolumn{2}{|c||}{} & Mag. & Angle & Mag. & Angle & Mag. & Angle \\
\hline \hline \multirow{2}{*}{$\mathrm{V}_{3}$} & Y-g & 0.014840 & 88.34 & 0.013040 & -36.50 & 0.013130 & -151.57 \\
\cline { 2 - 8 } & $\Delta$ & 0.009119 & 81.72 & 0.007125 & -40.78 & 0.007895 & -168.40 \\
\hline$I_{3}$ & Y-g & 0.009088 & 48.31 & 0.012530 & -71.72 & 0.010850 & 170.14 \\
\cline { 2 - 7 } & $\Delta$ & 0.005586 & 41.69 & 0.006848 & -76.00 & 0.006523 & 153.31 \\
\hline
\end{tabular}

(4) Effects of saturation.

The effects of saturation are also studied using the simplified test system. The saturation curve is modelled as piecewise linear between the subsequent data points (MMF, $\lambda$ ) $=(0.9,0.9) ;(1.5,1.2) ;(2.7,1.8)[38]$. Table 5.4 lists the harmonic profiles of the machine. The results obtained without saturation are also listed. 
Table 5.4: Voltage and current harmonics with and without saturation.

\begin{tabular}{|c|c||rr|rr|rr||c|}
\hline \multicolumn{2}{|c||}{ Item } & \multicolumn{2}{c|}{$\mathrm{h}=1$} & \multicolumn{2}{c|}{$\mathrm{h}=3$} & \multicolumn{2}{c|}{$\mathrm{h}=5$} & \multirow{2}{*}{$\%$ dif. } \\
\cline { 3 - 9 } & & Mag. & Angle & Mag. & Angle & Mag. & Angle & \\
\hline \hline $\mathrm{N}$ & $\mathbf{V}_{\mathbf{a}}$ & 1.10300 & 1.19 & 0.01484 & 88.34 & 0.00020 & 148.47 & \\
$\mathrm{~S}$ & & 1.10300 & 1.18 & 0.01202 & 76.31 & 0.00014 & 115.81 & 19.00 \\
\hline $\mathrm{N}$ & $\mathbf{V}_{b}$ & 1.02500 & -121.51 & 0.01304 & -36.50 & 0.00017 & 23.80 & \\
$\mathrm{~S}$ & & 1.02500 & -121.50 & 0.01061 & -48.43 & 0.00012 & -8.87 & 18.63 \\
\hline $\mathrm{N}$ & $\mathbf{V}_{\mathrm{c}}$ & 1.02200 & 120.24 & 0.01313 & -151.57 & 0.00017 & -90.29 & \\
$\mathrm{~S}$ & & 1.02200 & 120.23 & 0.01068 & -163.78 & 0.00013 & -122.95 & 18.66 \\
\hline \hline $\mathrm{N}$ & $\mathrm{I}_{a}$ & 0.00272 & 167.30 & 0.02253 & -152.50 & 0.00022 & -99.54 & \\
$\mathrm{~S}$ & & 0.00221 & 168.55 & 0.01824 & -164.53 & 0.00014 & -131.31 & 19.04 \\
\hline $\mathrm{N}$ & $\mathrm{I}_{b}$ & 0.00272 & -72.70 & 0.02249 & 87.45 & 0.00022 & 140.46 & \\
$\mathrm{~S}$ & & 0.00221 & -71.45 & 0.01829 & 75.53 & 0.00014 & 108.69 & 18.67 \\
\hline $\mathrm{N}$ & $\mathbf{I}_{c}$ & 0.00272 & 47.30 & 0.02250 & -32.44 & 0.00022 & 20.46 & \\
$\mathrm{~S}$ & & 0.00221 & 48.55 & 0.01828 & -44.64 & 0.00014 & -11.31 & 18.76 \\
\hline
\end{tabular}

$\mathrm{N}$ - without saturation, $\mathrm{S}$ - with saturation.

\%dif. - relative difference of the 3rd harmonic magnitude between $\mathrm{N}$ and $\mathrm{S}$.

As indicated in the last column, the difference between the cases with and without saturations are large. The differences in the phase angles are even more noticeable. This observation suggests that for some cases including the saturation is important to get more accurate results.

(5) Cases with other harmonic sources.

The effects of machine nonlinearities are further investigated by considering the external harmonic sources. To facilitate the interpretation of the results, only simplified operating conditions are studied. In this case, the external harmonics are represented as 3rd and 5th current injections to the system as shown in Figure 5.4, and the network is in a grounded $\mathrm{Y}$ connection. The results are listed in Table 5.5. Three types of machine modelling, namely, no machine nonlinearities, with frequency conversion only, and with both frequency conversion and saturation are analyzed. These results further confirm 
the need to model machine nonlinearity in detail.

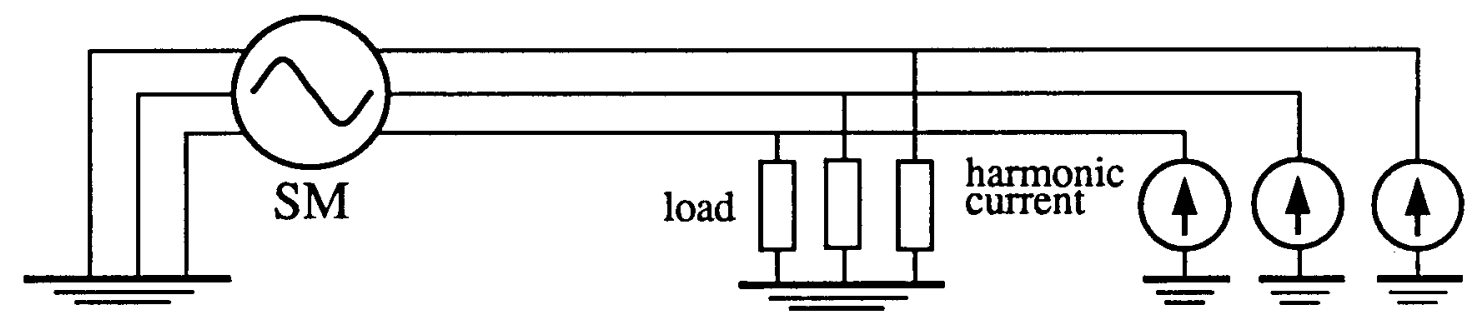

Figure 5.4: One machine test system with other harmonic sources.

Table 5.5: Machine 1 voltages with other harmonic sources.

\begin{tabular}{rl||cc|cc|cc|l|}
\hline \multirow{2}{*}{} & \multicolumn{2}{|c|}{ Phase-A } & \multicolumn{2}{c|}{ Phase-B } & \multicolumn{2}{c|}{ Phase-C } & \multirow{2}{*}{ Model } \\
\cline { 2 - 7 } & Mag. & Angle & Mag. & Angle & Mag. & Angle & \\
\hline \hline \multirow{3}{*}{$\mathrm{h}=1$} & 1.09900 & $\mathbf{0 . 2 8}$ & 1.02300 & -120.82 & 1.02800 & 120.52 & $\mathrm{~L}$ \\
& 1.10000 & $\mathbf{0 . 3 4}$ & 1.02400 & -120.90 & 1.02600 & 120.53 & $\mathrm{~F}$ \\
& 1.10000 & $\mathbf{0 . 3 4}$ & 1.02400 & -120.88 & 1.02600 & 120.52 & $\mathrm{~F}+\mathrm{S}$ \\
\hline \multirow{3}{*}{$\mathrm{h}=3$} & 0.06074 & 69.42 & 0.03042 & -171.54 & 0.05361 & -68.41 & $\mathrm{~L}$ \\
& 0.07777 & 67.31 & 0.02834 & -155.30 & 0.05724 & -82.96 & $\mathrm{~F}$ \\
& 0.07244 & 66.43 & 0.02953 & -156.70 & 0.05263 & -79.72 & $\mathrm{~F}+\mathrm{S}$ \\
\hline \multirow{3}{*}{$\mathrm{h}=5$} & 0.03365 & 69.71 & 0.04504 & -59.23 & 0.03795 & 173.58 & $\mathrm{~L}$ \\
& 0.04459 & 80.13 & 0.05390 & -50.01 & 0.04585 & -173.62 & $\mathrm{~F}$ \\
& 0.04174 & 76.81 & 0.05170 & -52.91 & 0.04392 & -177.43 & $\mathrm{~F}+\mathrm{S}$ \\
\hline
\end{tabular}

\subsubsection{Comparison with Transient Simulations}

An alternative method for obtaining distorted steady-state is to perform a transient simulation with EMTP that starts from the approximate linear ac steady-state conditions. The traditional machine model described in Chapter 3 is used for this ac solution. In this 
transient simulation, the only disturbances will then be the deviations resulting from harmonic components. The transients caused by these deviations may settle down quickly to the distorted steady-state if the system is well-damped.

The performance of the MHLF technique and its compatibility with EMTP can be evaluated by comparing the results from MHLF with those from transient simulations for some cases where the steady-state can be reached quickly. For this purpose, an ideal unbalance case was designed. In this case, the grounded $\mathrm{Y}$ connected machine 1 is supplied with a three-phase $\mathrm{Y}$ grounded RLC load. Phase A load is a 1pu resistive load, phase B a 1pu inductive load and phase C a 1pu capacitive load. The MHLF was run for this case with up to 7 th harmonics included. The program converged quickly to the final harmonic solution. Typical results are plotted in Figure 5.5, which shows the current and voltage waveforms of phase C.

It can be seen from the figure that the transients in EMTP simulation disappear quickly for this case. Once the transients are over, MHLF results match very well with the waveforms from EMTP. This is what is expected from a theoretical point of view, since both approaches adopt the same machine model and assumptions. Further inspection indicates that the waveform differences caused by numerical digitization in EMTP and harmonic truncation in MHLF are not noticeable. All these observations suggest that the MHLF technique is accurate and efficient. Its results are suitable to initialize the EMTP simulations.

\subsubsection{Comparison with Theoretical Results}

In the traditional three-phase load flow analysis, the negative sequence impedance of synchronous machines is important for the modelling of machine performance under unbalanced conditions. The value of this impedance is somewhat arbitrary, however, in 


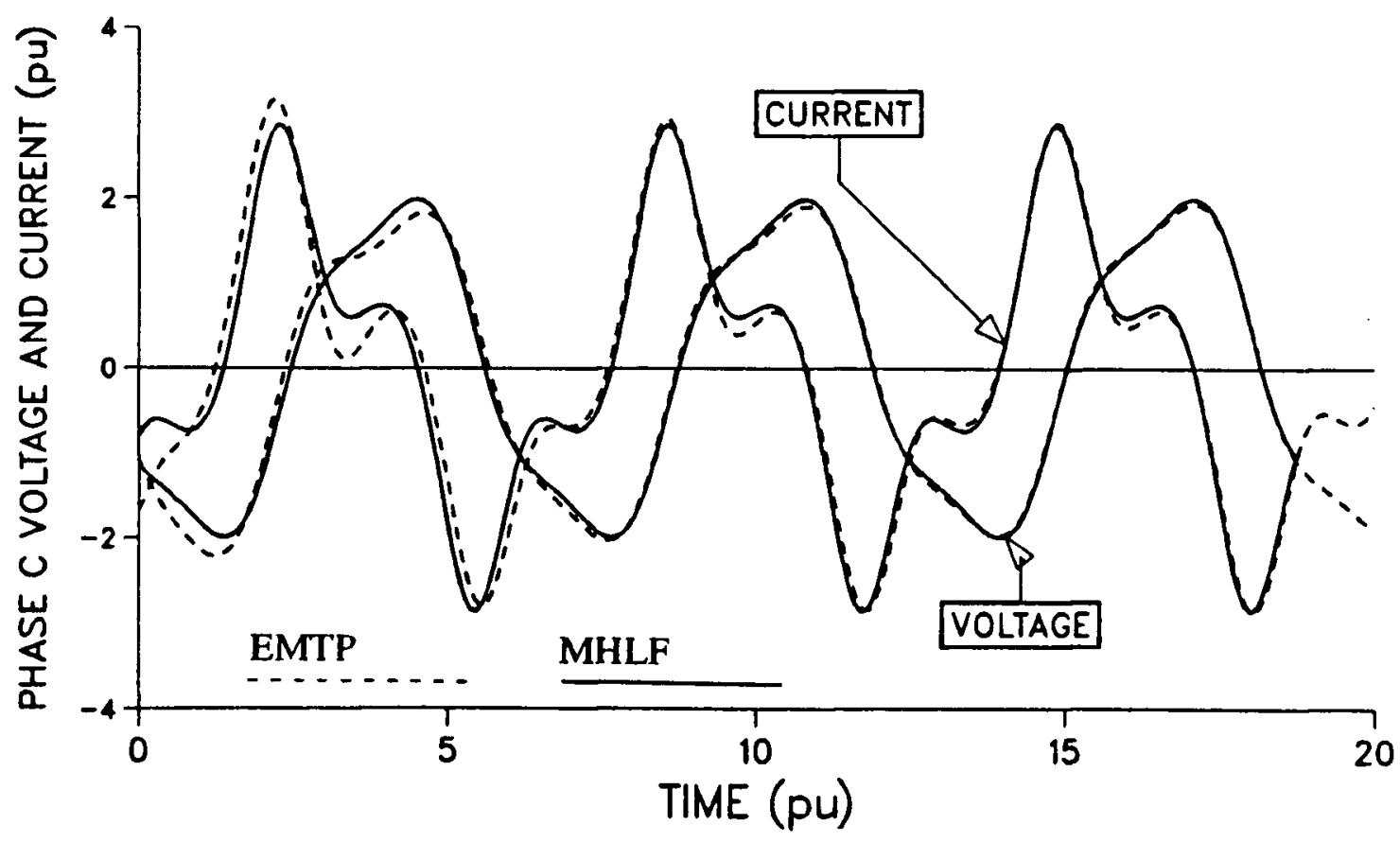

Figure 5.5: Comparison of waveforms from EMTP and MHLF.

the sense that it is different when the machine is supplied with negative sequence current (current- or I-definition) or voltage (voltage- or V-definition) [38]. This arbitrariness may lead to inconsistent conclusions regarding, for instance, the load flow distributions of salient pole machines.

This inconsistency was identified to be caused by the frequency conversion process. In this section, the effects of frequency conversion on the negative sequence impedance is studied using the MHLF technique. Since theoretical results are available for the impedance calculations [38], the performance of MHLF technique can also be accurately assessed. The two test conditions commonly accepted to define machine negative sequence impedance (Figure 5.6) are used for these tests. The first case (Figure 5.6(a)) is to connect the machine to a negative sequence current source and record the negative sequence terminal voltage (I-definition). The second one (Figure $5.6(\mathrm{~b})$ ) is to connect a 
negative sequence voltage source and record the current (V-definition). The impedance is given by the ratio of the voltage to the current. The results obtained with the test machine 2 have been listed in Table 5.6.

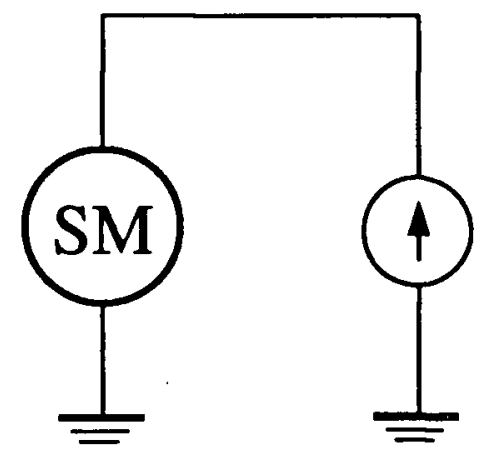

(a) current test

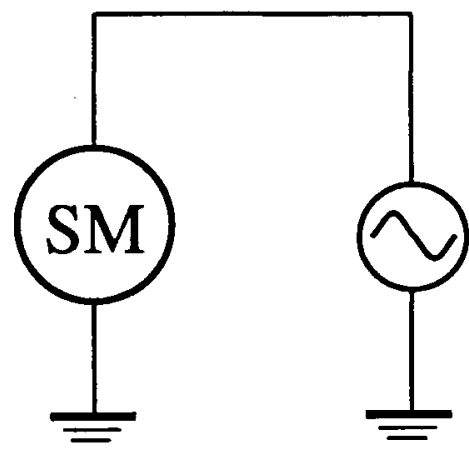

(b) voltage test

Figure 5.6: Two tests to determine machine negative sequence impedance.

Table 5.6: Values of negative sequence impedance.

\begin{tabular}{|c|r||r|r|r|}
\hline Tests & Theoretical & \multicolumn{1}{|c|}{$\mathrm{H}=1$} & $\mathrm{H}=1,3$ & $\mathrm{H}=1,3,5$ \\
\hline \hline current & 0.0655 & 0.0857 & 0.0655 & 0.0655 \\
definition & $+\mathrm{j} 0.3067$ & $+\mathrm{j} 0.2165$ & $+\mathrm{j} 0.3066$ & $+\mathrm{j} 0.3066$ \\
\hline voltage & $\mathbf{0 . 0 8 5 8}$ & $\mathbf{0 . 0 8 5 6}$ & $\mathbf{0 . 0 8 5 9}$ & $\mathbf{0 . 0 8 5 9}$ \\
definition & $+\mathrm{j} 0.2165$ & $+\mathrm{j} 0.2166$ & $+\mathrm{j} 0.2164$ & $+\mathrm{j} 0.2164$ \\
\hline
\end{tabular}

$\mathrm{H}=1$ - Only the fundamental frequency component included.

$\mathrm{H}=1,3-\mathrm{H}=1$ modelling plus the third harmonic.

$\mathrm{H}=1,3,5-\mathrm{H}=1,3$ modelling plus the fifth harmonic.

As shown in this table, the program was run for three different levels of modelling. The second column displays the exact impedance values computed from theoretical formulas. The following observations are obtained: 
1. The difference between the machine impedances calculated from I- and V- definitions is caused by the 3 rd harmonic. The contribution of 5 th harmonic is negligible.

2. If harmonics are not included, the MHLF technique exhibits the behavior of the machine models of V-definition.

3. In general unbalanced cases, the machine negative sequence impedance is neither equal to that of V-definition nor equal to that of I-definition. The machine response to such cases can only be fully evaluated with harmonics included. The integration of the load flow constraints with harmonic solutions is therefore the best approach in such situations.

4. The validity of the MHLF modelling and solution techniques is again justified by the close agreement with the theoretical results.

\subsubsection{Observations on the Convergence Behavior}

The convergence behavior of the program was investigated with the test cases in previous sections. A network with eight machines was also studied (see Figure 5.7). The converging process for all cases is shown in Table 5.7. This table shows the maximum update of the equivalent current sources representing the nonlinear effects. Also listed in the table is the computer times (CPU) used in all cases. It is noted that only 5 to 8 iterations are needed for all the cases with convergence criteria $0.0001 \mathrm{pu}$. The rate of convergence is found to be linear, which is expected from theoretical point of view. These observations are consistent with those made in nonlinear inductor studies. The number of subiterations used for solving the saturation was found between 7 and 15 . 


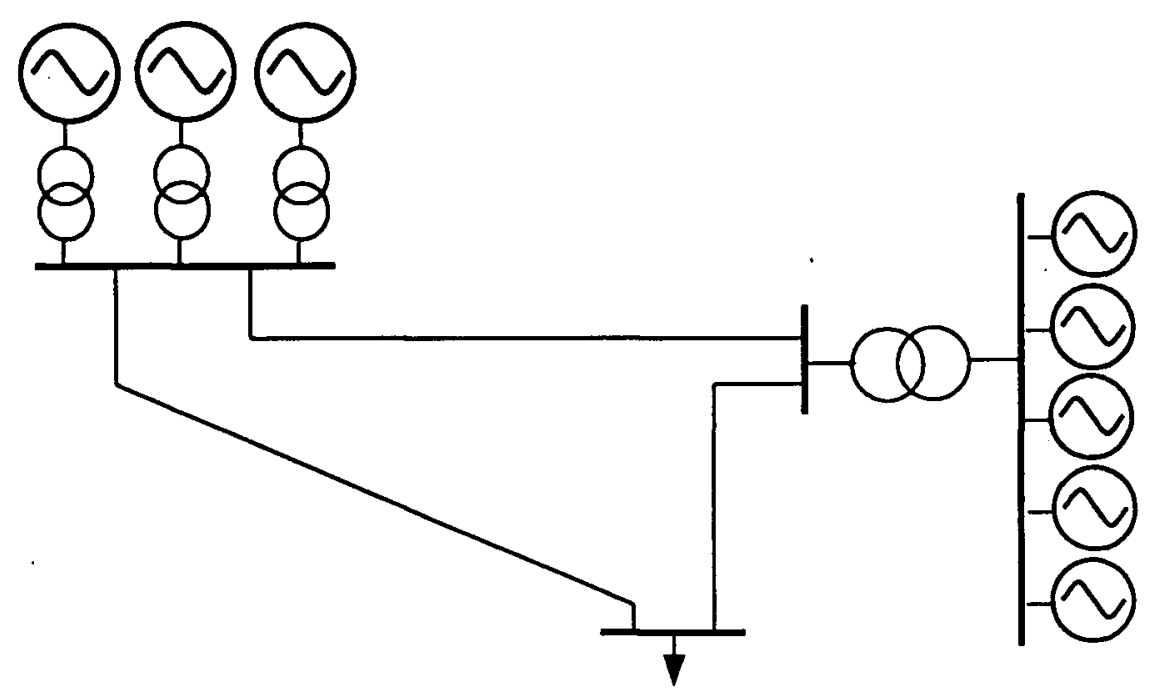

Figure 5.7: A multimachine test system.

Table 5.7: Convergence behavior of the MHLF technique.

\begin{tabular}{|c|c|c|c|c|c|}
\hline \multirow{2}{*}{$\begin{array}{c}\text { Iteration } \\
\#\end{array}$} & \multicolumn{5}{|c|}{ Maximum update of machine harmonic current source } \\
\hline & Case 1 & Case 2 & Case 3 & Case 4 & Case 5 \\
\hline$\overline{1}$ & $\overline{0.02202}$ & $\overline{0.05774}$ & 0.01798 & 0.01592 & 0.07770 \\
\hline 2 & 0.00259 & 0.02101 & 0.00219 & 0.00201 & 0.02323 \\
\hline 3 & 0.00046 & 0.00982 & 0.00034 & 0.00031 & 0.00848 \\
\hline 4 & 0.00006 & 0.00364 & 0.00004 & 0.00004 & 0.00261 \\
\hline 5 & & 0.00167 & & & 0.00108 \\
\hline 6 & & 0.00063 & & & 0.00030 \\
\hline 7 & & 0.00030 & & & 0.00011 \\
\hline 8 & & 0.00008 & & & 0.00003 \\
\hline $\mathrm{CPU}(\mathrm{s})$ & 2.49 & 4.92 & 6.81 & 7.21 & 69.88 \\
\hline $\begin{array}{l}\text { Case 1: } \\
\text { Case 2: } \\
\text { Case 3: } \\
\text { Case 4: } \\
\text { Case 5: }\end{array}$ & $\begin{array}{l}\text { Machine } 1 \\
\text { Machine } 2 \\
\text { Case } 1 \text { plus } \\
\text { Case } 3 \text { plus } \\
\text { Multimachi }\end{array}$ & $\begin{array}{l}\text { th unbala } \\
\text { th the san } \\
\text { aturation } \\
\text { xternal ha } \\
\text { test case }\end{array}$ & $\begin{array}{l}\text { ed load ( } \\
\text { load as } \\
\text { ect (Fig } \\
\text { nonic cur } \\
\text { Pigure } 5 .\end{array}$ & $\begin{array}{l}\text { sure 5.3) } \\
\text { e } 1 \text { (Figu } \\
5.3 \text { ). } \\
\text { it source }\end{array}$ & (re 5.4 ). \\
\hline
\end{tabular}




\subsection{Summary}

A detailed synchronous machine model has been developed in this chapter. Two nonlinear effects, the frequency conversion and saturation, are included. A comprehensive MHLF solution process is formed by including these effects with three type machine load flow constraints, thus allowing a systematic harmonic analysis for a wide range of operating conditions. The comparisons of MHLF results with both EMTP simulation and theoretical solution have demonstrated the usefulness and efficiency of the MHLF technique.

Results from the respective case studies indicated that there are noticeable harmonic distortions caused by the unbalanced operation of machines, even the degree of unbalance is within their operating limitations. Major observations of these studies can be summarized as the follows:

1. The results from MHLF program are compatible with those from EMTP simulations. The MHLF technique is therefore a suitable candidate for EMTP initializations.

2. The fundamental frequency load flow results may not be correct due to the ambiguous value of the machine negative sequence impedance. For such cases, the correct results can only be obtained by including harmonics in the load flow solution.

3. Only 3rd and 5th harmonics need to be included for machine harmonic analysis. The 3rd harmonic is in negative sequence and not in zero sequence. Hence, it can not be elimináted by $\Delta$-connections.

4. Machine saturation can have noticeable effects on the harmonic distributions. These effects are more significant with respect to the harmonic phase angles. 


\section{Chapter 6}

\section{Unbalanced Harmonic Analysis of Static Compensators}

With the multiphase harmonic load flow (MHLF) technique developed in previous chapters, it is now possible to analyze the unbalanced harmonics generated by static compensators. The necessary steps to accomplish such analysis are presented in this chapter. A general scheme for considering the compensator control characteristics in the harmonic solution is described. In effect, this chapter illustrates a complete procedure of using the MHLF technique for the harmonic analysis of a general nonlinear elements with specific control characteristics.

\subsection{Frequency-domain Model of Thyristor-Controlled Reactor}

It has been known that the harmonic source in the static compensators under study is the thyristor-controlled reactor (TCR). As the first step of harmonic analysis with the MHLF technique, the frequency-domain model of this nonlinear element shall be developed.

\subsubsection{Harmonic Norton Equivalent Circuit of TCR}

According to the development in Chapter 2, the TCR can always be represented as a harmonic current source of the following form:

$$
i_{\text {harmonic }}(t)=\sum_{h=1}^{n} \sqrt{2}\left|\mathbf{I}_{h}\right| \cos \left(h \omega t+\theta_{h}\right) .
$$

This current is obtained by a simple numerical process with a given harmonic voltage condition at the TCR terminal. Instead of replacing the TCR with the current source as 
in Chapter 2, it is better to model the TCR as a Norton equivalent circuit, however. It can be shown that the equivalent inductance of a TCR for a purely sinusoidal voltage at fundamental frequency is $[20]$ :

$$
L_{e q}=\pi L(\sigma-\sin \sigma)^{-1}
$$

where $L$ is the reactor inductance and $\sigma$ the known conduction angle. This equivalent inductance represents the TCR very well at fundamental frequency, and reasonably well at other frequencies. The differences between the current absorbed in $j h \omega L_{e q}$ and the actual current from Eq. (6.1) become the parallel current sources in the Norton equivalent circuit representation of Figure 6.1, with

$$
\begin{aligned}
& Y_{h-\text { eq }}=\left(j h \omega L_{e q}\right)^{-1} \\
& \mathbf{I}_{h-\text { eq }}=\left(j h \omega L_{\text {eq }}\right)^{-1} \mathbf{V}_{h}-\mathbf{I}_{h}
\end{aligned}
$$

where $\mathbf{V}_{h}=\left|\mathbf{V}_{h}\right| e^{j \phi_{h}}$ and $\mathbf{I}_{h}=\left|\mathbf{I}_{h}\right| e^{j \theta_{h}}$. Note that there is no coupling among the equivalent circuits of the different harmonic frequencies. They are also independent of any network unbalances.
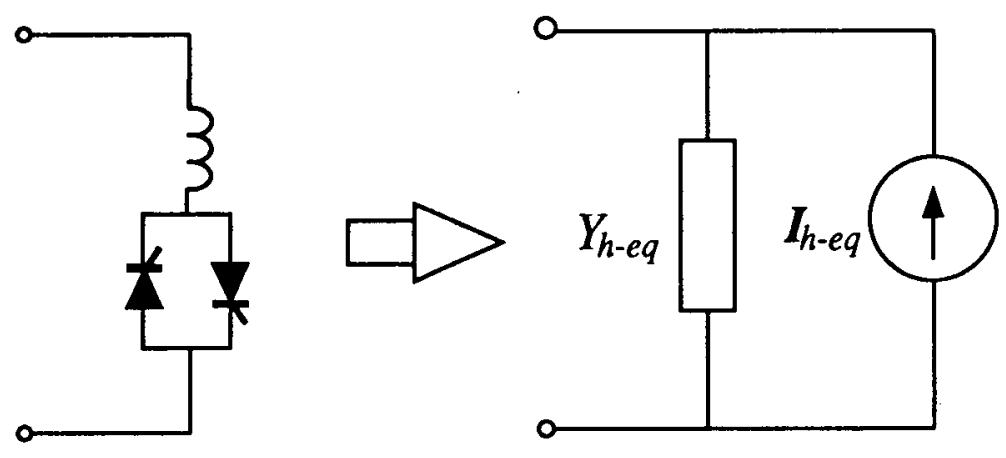

Figure 6.1: TCR model for multiphase harmonic analysis. 


\subsubsection{Considerations on the Practical Compensator Systems}

A practical static compensator, such as the one shown in Figure 2.2, is more complicated than the simple TCR unit of Figure 6.1(a). First of all, there is the delta connection of the TCR's. This connection is used to filter the zero sequence harmonics (e.g. 3rd, 9th). With the multiphase representation and the Norton equivalent circuits, the modelling of delta connection is straightforward, and the circulation of the zero sequence harmonic currents is automatically taken into account. When the operating conditions are unbalanced, the non-characteristic harmonics in the delta connection are automatically obtained.

The second complication is the three-winding transformer connection. The phase shifting effects of the connection are critical for the cancellation of the 5th and 7 th harmonics generated by the TCR's at the two secondary windings. With the multiphase modelling of transformers, this phase shift is automatically included [12]. The phase shifter model is no longer required. Since the voltage ratio can also be included in the transformer model, the load flow analysis can be performed in either per-unit or physical quantities.

\subsection{Interfacing with the MHLF Process}

With the Norton equivalent circuits just developed, each TCR can be replaced by an admittance in parallel with a harmonic current source. These representations are readily acceptable by the MHLF solution process. A multiphase load flow and harmonic frequency scan problem is then formulated. The problem is solved by the solution technique developed in Chapter 3 .

Similar to the Norton equivalent circuits for nonlinear inductors and synchronous machines, the parallel current source $\mathbf{I}_{h-e q}$ in the TCR equivalent circuit is only known approximately. It is estimated from the load flow results prior to the improved model 
being applied. An iterative process is therefore needed for accurate solution, as described in Chapter 4.

Compared with the current source representation for the TCR in Chapter 2, the advantages of Norton equivalent circuit are twofold. Firstly, the deviation of the initial load flow solutions from the final solutions is smaller, due to the presence of $L_{e q}$ in the network. Secondly, the Norton equivalent circuits can avoid possible singularity conditions caused by the current source models. Such conditions exist, for example, if the delta structured TCR's are directly connected to the transformer secondary winding without any grounding connections.

\subsection{Simulation of Static Compensator Control Characteristics}

During normal operation, the compensator operates according to its control characteristics. The TCR conduction angles and associated harmonic distributions are therefore dependent on the network load flow conditions. The MHLF technique has been developed to take the characteristics into account.

\subsubsection{Control Characteristics in Multiphase Setting}

The static compensator control characteristic has been described by a set of linear voltagecurrent conditions as shown in Figure 2.3 and expressed as

$$
\left|\mathbf{V}_{1}\right|=V_{s}+I_{r} k
$$

where

$V_{1}$ is the positive sequence fundamental frequency voltage at the compensator bus,

$V_{s}$ is the voltage set point, 
$I_{r}=\left|\mathbf{I}_{1}\right| \sin \left(\theta_{1}-\phi_{1}\right)$ is the reactive component of $\mathbf{I}_{1}$,

$k$ is the slope constant,

$I_{1}$ is the positive sequence fundamental frequency current injected into the network, and $\phi_{1}, \theta_{1}$ are the phase angles of $\mathbf{V}_{1}$ and $\mathbf{I}_{1}$, respectively.

It is assumed here that the characteristics are defined with respect to positive sequence quantities. The static compensator operates at the intersection of its control characteristic with the network characteristic, as shown in Figure 2.3. To include this constraint in the MHLF solution, a conduction angle adjustment scheme is developed, as described next.

\subsubsection{Conduction Angle Adjustment Scheme}

From the principle of the actual firing angle control, a control error can be defined as

$$
\varepsilon=\left|\mathbf{V}_{1}\right|-\left(V_{s}+I_{r} k\right)
$$

By varying the conduction angles, as shown in Figure $6.2(\mathrm{a})$, the value of $\varepsilon$ can be obtained as a function of $\sigma$ (Figure 6.2(b)). The correct conduction angle which meets the static compensator control characteristic is the one corresponding to $\varepsilon=0$ on the curve. To determine this angle, the secant method is applied to the $\varepsilon-\sigma$ curve [24]. The solution process is indicated in Figure 6.2(b), and can be expressed as

$$
\sigma_{i+1}=\sigma_{i}+\frac{\sigma_{i}-\sigma_{i-1}}{\varepsilon_{i}-\varepsilon_{i-1}} \varepsilon_{i}
$$

where $i$ is the iteration number. The complete solution of the TCR with both the conduction angle adjustment and the harmonic iteration can then be summarized as follows: 


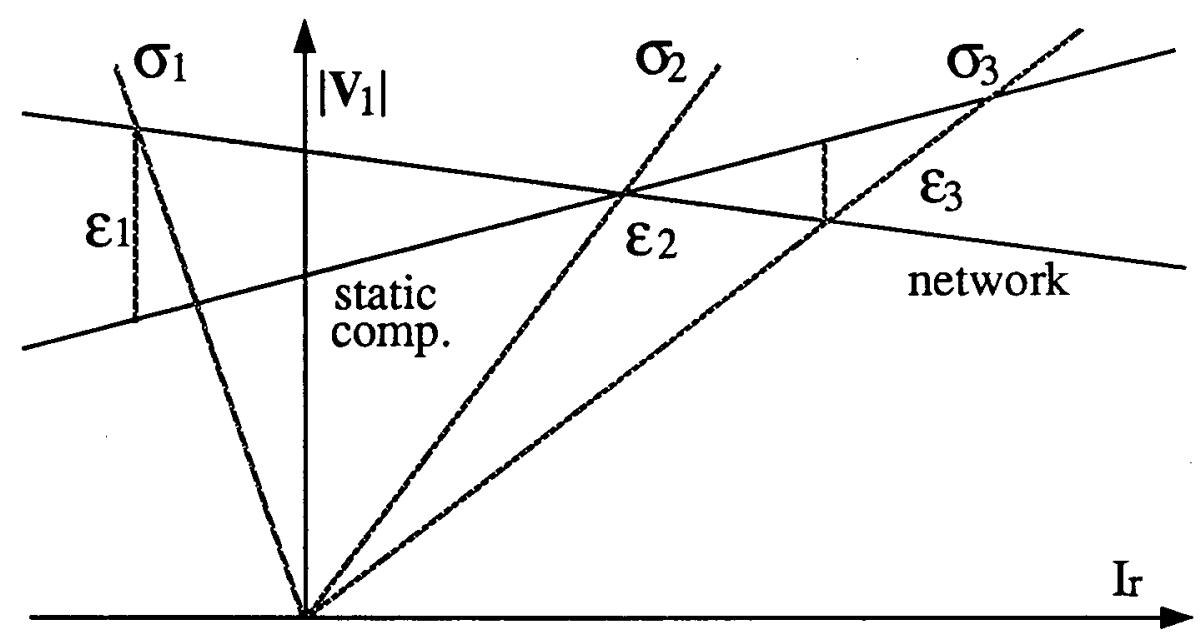

(a) static compensator control errors with conduction angles.

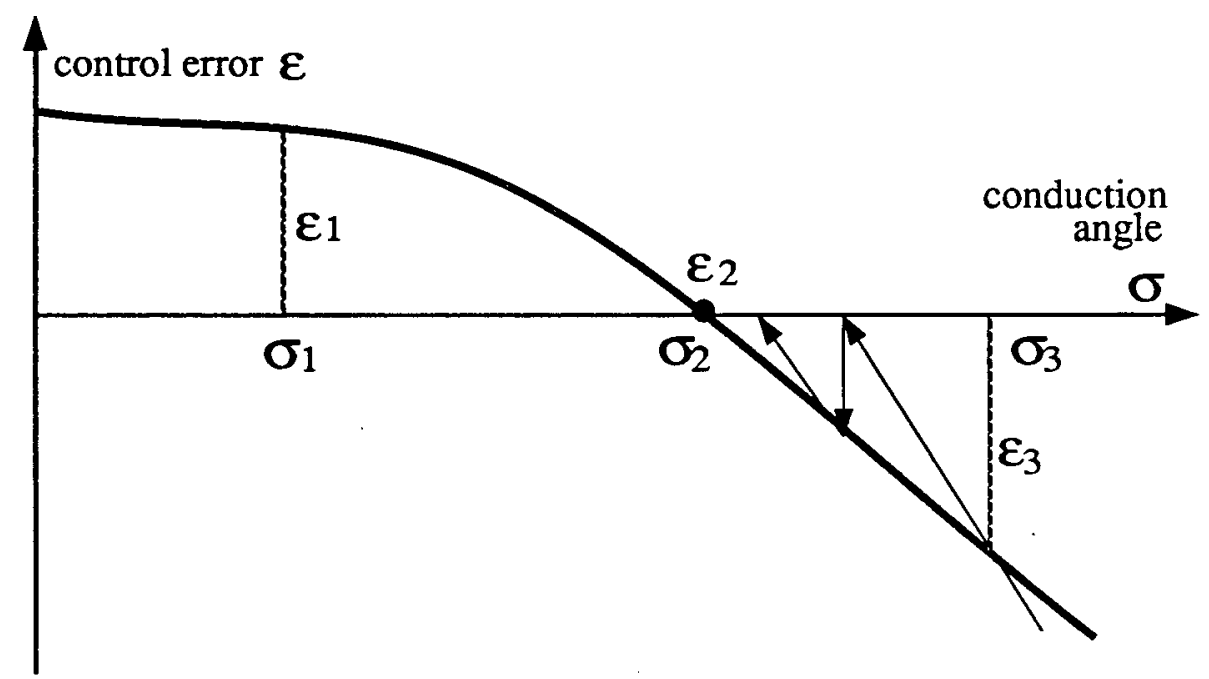

(b) control error as a function of conduction angle.

Figure 6.2: Solution of conduction angles. 
Step 1. Initialization.

Represent TCR's as Norton equivalent circuits with their parallel harmonic current sources set to zero. The equivalent circuits are computed for the given initial conduction angles.

Step 2. Network solution.

Replace the TCR's with the equivalent circuits and perform the multiphase fundamental frequency load flow solution and frequency scan.

Step 3. Conduction angle adjustment.

Calculate the control error $\varepsilon$ for the particular load flow solution, and find the new conduction angle with the method depicted in Figure 6.2.

Step 4. Norton equivalent circuit computation.

Recompute the TCR equivalent circuits with the newly obtained conduction angles and the TCR terminal voltage conditions.

Step 5. Convergence check.

If the new Norton equivalent circuits are sufficiently close to the previous ones, the solution has converged. Otherwise, return to Step 2.

This conduction angle adjustment scheme is much simpler than the one developed in Chapter 2. It does not require the estimation of network characteristics and resembles closely the actual voltage regulating process of the static compensator [20]. Other compensator configurations and control characteristics can be analyzed in a similar way. The only difference would be in the computation of the control error of Eq. (6.6). 


\subsection{Convergence Properties}

To analyze the convergence property of the MHLF technique with static compensators, both the iteration process for the harmonic components and the adjustment of TCR conduction angles must be considered.

In the iterative solution for harmonic components, no convergence difficulties have been experienced. This is partly due to the low pass filter effects of the TCR. Because of these effects, the harmonic voltage changes at the TCR terminal do not influence the equivalent harmonic currents significantly. Secondly, the network harmonic characteristic as a passive impedance and the TCR as a harmonic current source with a large impedance in parallel also improve the convergence performance. As for the solution at fundamental frequency, the Norton equivalent circuit can represent the TCR more accurately compared with a simpler current source representation. This also helps the convergence.

The iteration for the conduction angle is simply a single variable problem when there is only one static compensator in the network. Generally, the correct conduction angle can easily be obtained by the secant method. The direct solution of the conduction angle from the $\varepsilon-\sigma$ curve of Figure 6.2 avoids the convergence difficulties of the simpler approach in Chapter 2. If there are more compensators in the network, the interactions among the conduction angles are reflected on the network line, which represents the equivalent network impedance seen from each compensator location. These interactions are not strong since the reactive power generated by one compensator is not much influenced by the reactive power of a compensator somewhere else in the system. Consequently, the convergence performance of the $\varepsilon-\sigma$ iteration is not significantly affected by the presence of other compensators.

It needs to be noted that the two iteration schemes work in parallel to each other 
in the actual solution process. The above analysis is still applicable, however, due to two facts. Firstly, the conduction angle adjustment is mainly associated with the fundamental frequency component. The coupling of the two processes is not strong therefore. Secondly, the conduction angle converges in the first stage which is then followed by the convergence of harmonic iterations.

\subsection{Case Studies and Applications}

To illustrate the performance and application of the MHLF program for power systems with static compensators, various cases were studied. Major results of the investigations are presented in this section.

\subsubsection{Field Measurement Comparisons}

The correctness of the MHLF program for static compensators is first assessed by comparing the computed results with the field measurements. Again, the TransAlta Utilities Corporation system is selected for this purpose. Same measurements used in Chapter 2 are considered for making the comparisons. In this case, the network is represented in three-phase form. It was found that the computed waveforms are the same as those from the single-phase program of Chapter 2. The correctness of the multiphase technique is then confirmed by the close agreement with the field records as well as with the single-phase solutions.

\subsubsection{Investigation of Non-characteristic Harmonics}

Under ideally balanced operating conditions, the triple order harmonics from the TCR's are filtered by the delta connection, and the 5th, 7th, 17th and 19th harmonics from the two secondary windings are cancelled by each other due to the wye-delta transformer 
connection. In practice, there always exists some degree of unbalance in the system. Consequently, these and other non-characteristic harmonics will appear at the compensator bus.

To evaluate the penetration of these harmonics into the network, three typical unbalanced conditions were investigated for the compensator shown in Figure 6.3. The compensator data are listed in Appendix E.

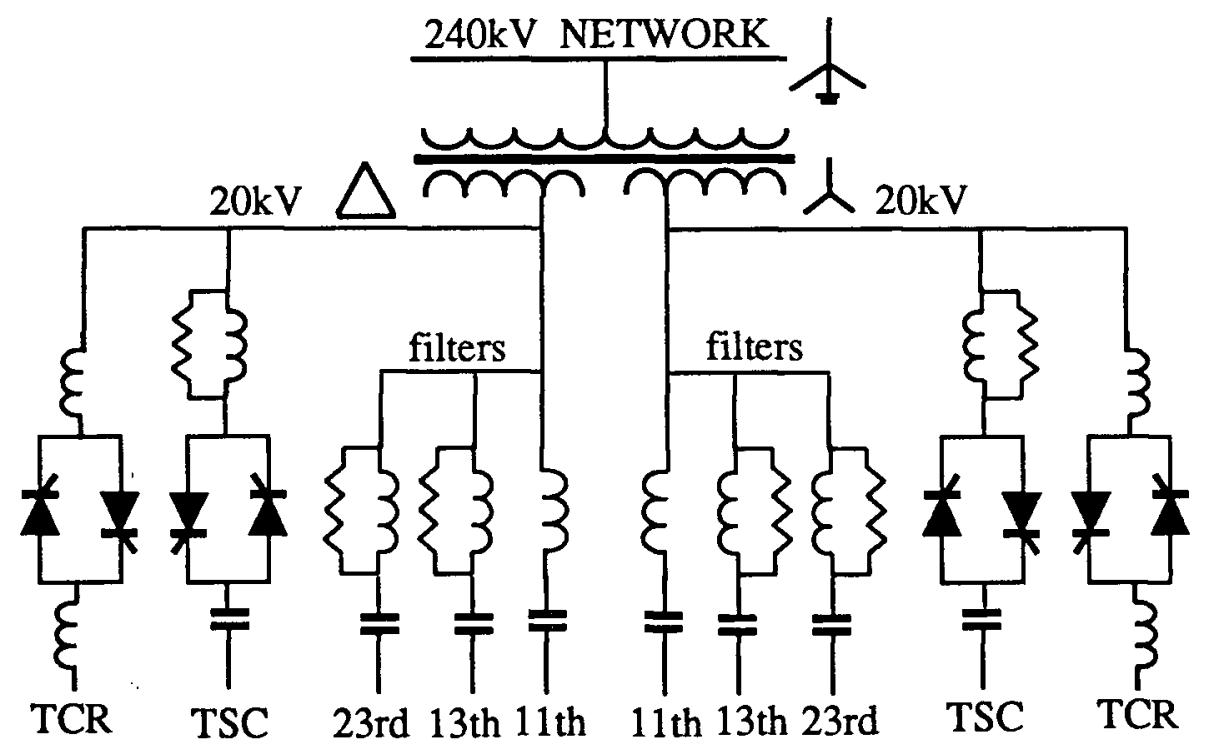

Figure 6.3: A typical static compensator.

(1) Effects of network voltage unbalance

To study the effects of voltage unbalances, it is assumed that the voltage at the compensator bus contains a negative sequence component, but that no harmonics are present in the voltage. The ratio of the negative to the positive sequence voltage $\left(V_{n} / V_{p}\right)$ is used to define the degree of voltage unbalance. Only the magnitude of the ratio is important. The relative phase angle between positive and negative sequence voltages 
had no effect on the harmonic spectrum. Figure 6.4 illustrates the percentage of harmonic currents as a function of the voltage unbalance. This percentage is defined as the ratio of the harmonic current to the positive sequence fundamental frequency current component. A conduction angle of $120^{\circ}$ was selected since it results in the largest 3rd harmonic generation of the TCR [20].
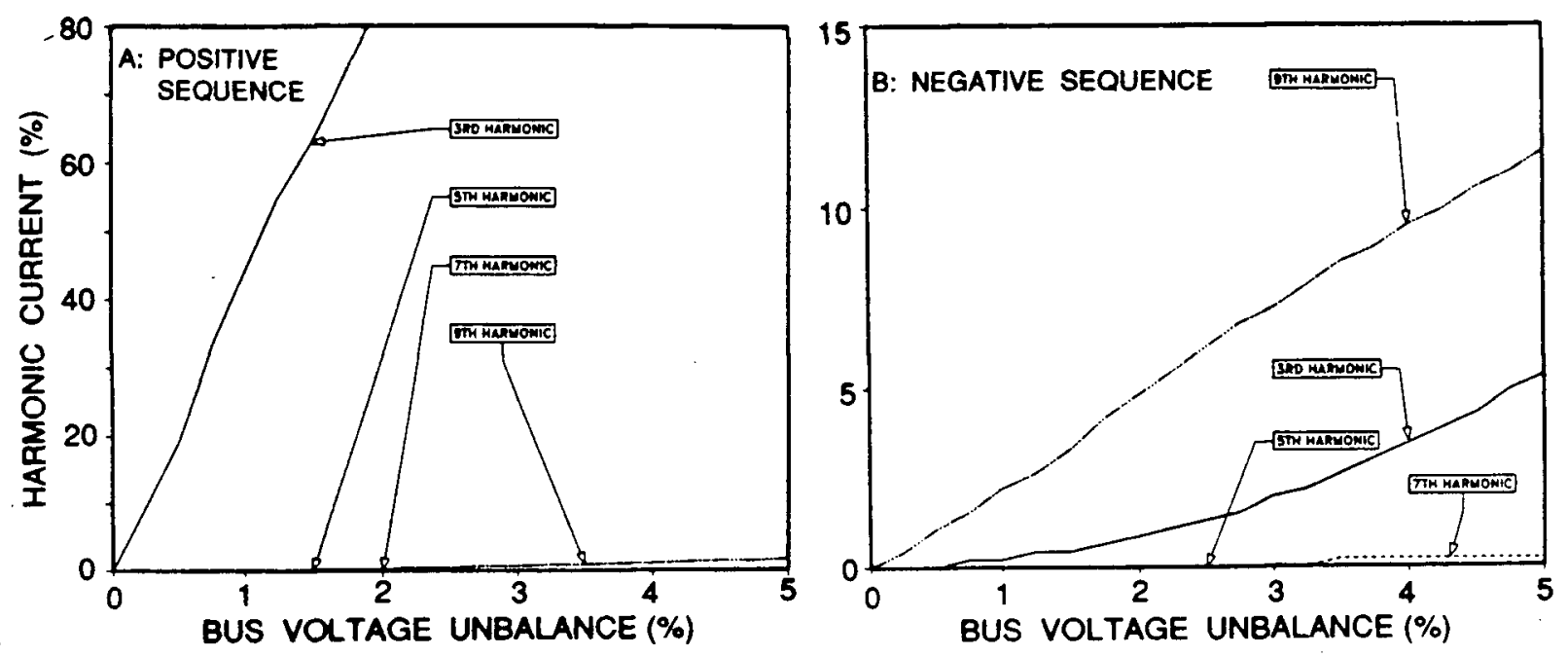

Figure 6.4: Harmonic current injection as a function of the compensator bus voltage unbalance.

The following conclusions can be drawn from these results:

1. The non-characteristic harmonic injection is proportional to the terminal voltage unbalance. Both negative and positive sequence harmonic currents are present. These harmonics are insensitive to the relative phase angle between the positive and negative sequence components of the fundamental frequency voltage.

2. The harmonic cancellation scheme of the three-winding transformer is still effective for the cancellation of the 5th and 7th harmonics. Further studies showed that this 
scheme also effectively cancels the negative sequence 3 rd harmonic and the positive sequence 9 th harmonic.

3. The percentage of $3 \mathrm{rd}$ harmonic injection is very high in the test case, because of a third harmonic current resonance in the compensator network. If the shunt capacitors are removed, the resonance disappears and the third harmonic injections become very small (approximately $2 \%$ ).

With the conduction angle changed from $0^{\circ}$ to $180^{\circ}$ and a voltage unbalance of $2 \%$, the percentage of harmonic current injections is plotted in Figure 6.5. These figures confirm the previous observations and indicate that $120^{\circ}$ is the worst case.
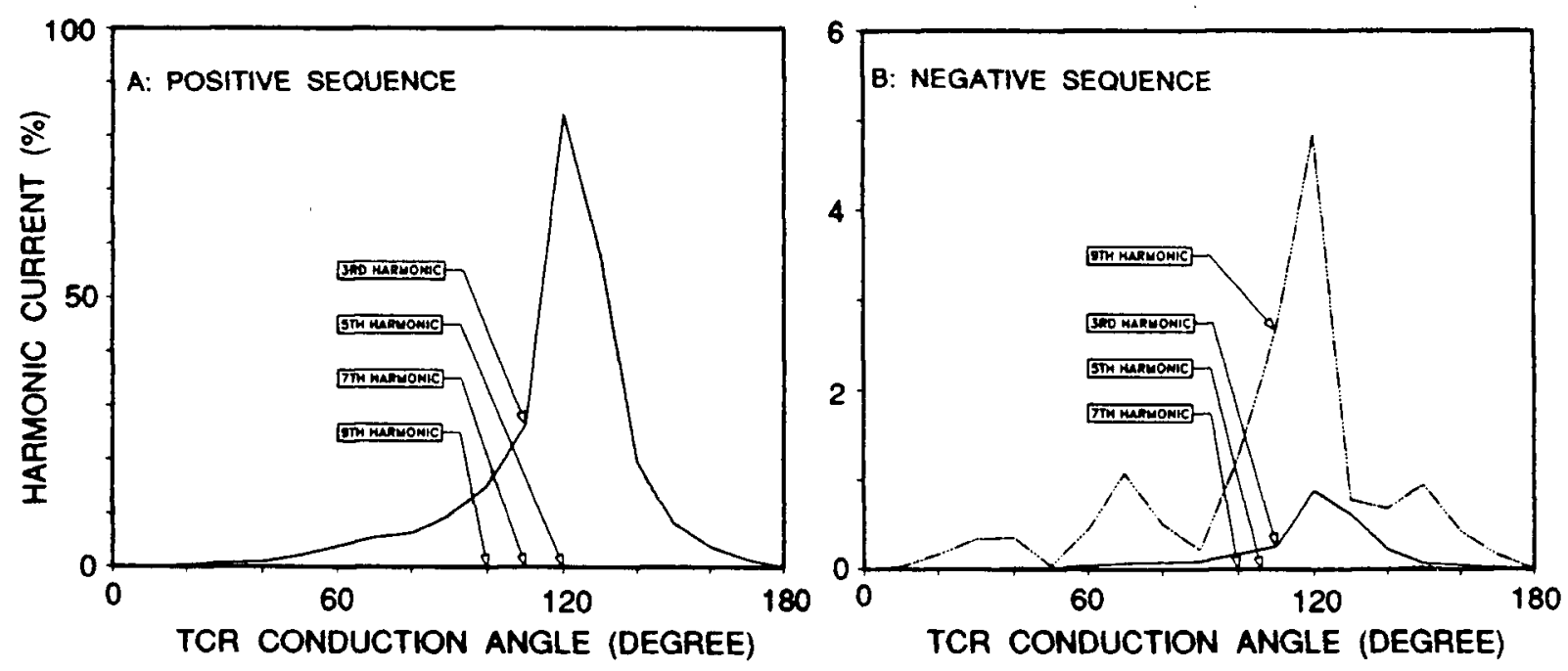

Figure 6.5: Harmonic current injection as a function of conduction angle.

(2) Effects of the TCR firing angle asymmetry

For a variety of reasons, it is not always possible to have identical firing angles for all six TCR's in the compensator of Figure 6.3. Differences of $2^{\circ}$ to $3^{\circ}$ are generally regarded as acceptable. To assess the impact of such firing angle asymmetry, it is assumed that 
only one TCR (phase A, delta side) has different conduction angles, changing from $115^{\circ}$ to $125^{\circ}$, while the other five TCR's have a fixed conduction angle of $120^{\circ}$. The rest of the system is assumed to be balanced. Figure 6.6 demonstrates the effects of firing angle asymmetry on the positive and negative sequence harmonic current injections.
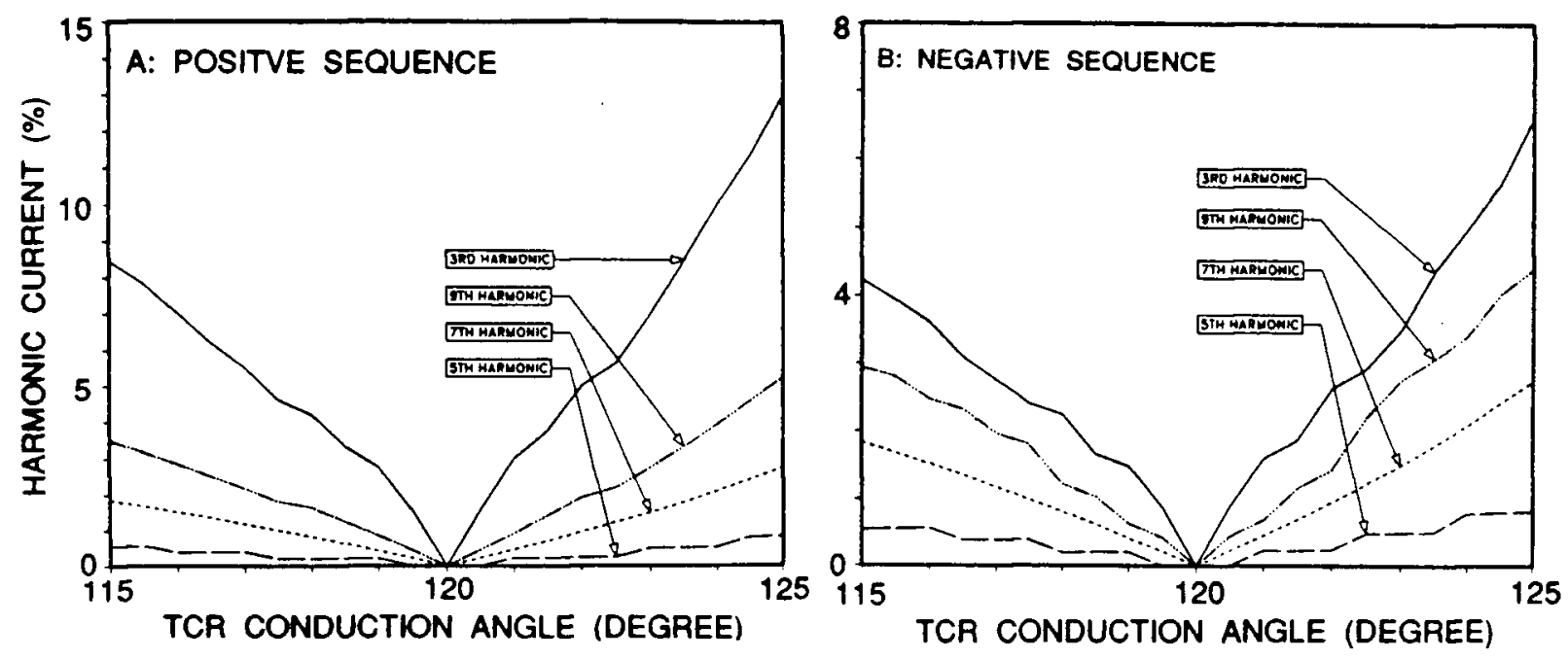

Figure 6.6: Harmonic current injection as a function of firing asymmetry.

(3) Effects of the TCR reactor unbalance

Another possible unbalance which cannot be completely eliminated is the difference in the reactance values of the six TCR reactors. A manufacturing tolerance of $2 \%$ to $5 \%$ is typical. The impact of this unbalance is assessed by assuming that only one TCR reactance (Phase- $A$, delta side) is different between $-5 \%$ to $5 \%$, while the other TCR reactances are identical. The rest of the system is balanced. The percentage magnitudes of the positive and negative sequence harmonic currents with respect to various degrees of reactor unbalance are plotted in Figure 6.7.

The results obtained with the firing angle asymmetry and TCR reactor unbalance suggest that: 

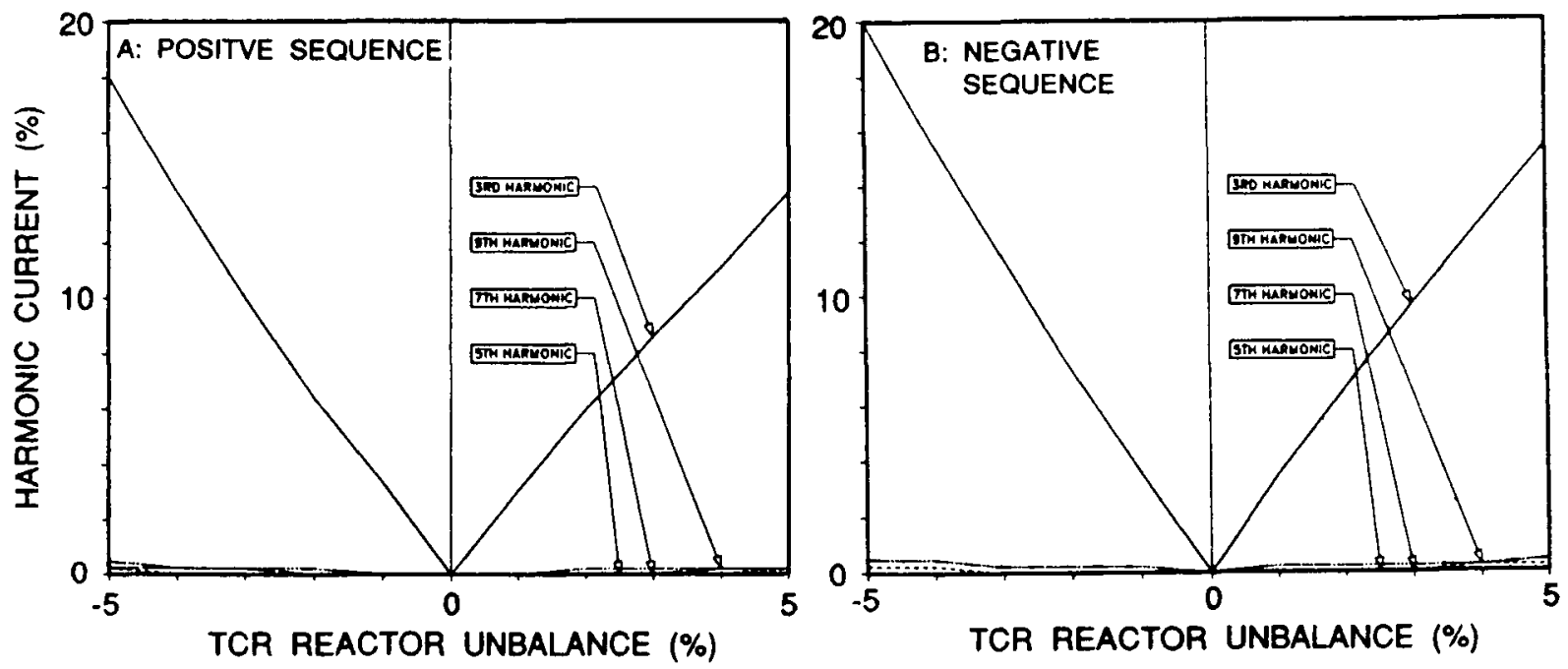

Figure 6.7: Harmonic current injection as a function of TCR reactor unbalance.

1. Both asymmetrical conditions can result in noticeable non-characteristic harmonic current injections. The harmonics caused by reactor unbalance are proportional to the degree of unbalance, while those by firing asymmetry are only approximately linear with respect to the degree of asymmetry.

2. The transformer connection is not very effective in cancelling the 5th and 7 th harmonic currents caused by firing asymmetry. But this cancellation scheme is still useful in the case of reactor unbalance.

3. As expected, the third harmonic current resonance also appears in these cases. However, the positive and negative sequence components are of the same order in both unbalanced conditions. This is caused by the unsuccessful cancellation of the negative sequence third harmonic current.

In all non-characteristic harmonic tests, there are no zero sequence harmonic currents in the $20 \mathrm{kV}$ and $240 \mathrm{kV}$ busses because of the TCR delta connection. 


\subsubsection{Harmonic Load Flow Solutions}

In practice, it is likely that several unbalanced conditions occur at the same time. The MHLF program is well suited for such studies, because it allows the modelling of various unbalance conditions, together with static compensator control characteristics. Such studies were made with the following examples:

1. The 19 bus TransAlta Utilities Corporation system with one compensator [25] (data are listed in Appendix E).

2. A 9 bus system, which is a reduced equivalent circuit of the above system. The 11th, 13th and 23rd harmonic filters were removed in this case.

3. A modified IEEE 14 bus (three-phase) test system [26], with two static compensators. Each compensator is assumed to have one two-winding transformer with one TCR set. There are no filters present in this case.

In these studies, all systems are represented in three-phase form. The unbalances are caused by transmission lines, loads, equivalent source voltages and TCR reactances. The TCR conduction angles are adjusted by the program to satisfy the control characteristics. Solutions were obtained up to the 15 th harmonic.

(1) Voltage harmonic spectrum.

The harmonic voltage spectra at the compensator bus are shown in Figure 6.8. Figure 6.9 shows the harmonic spectra of the bus voltage where the TCR's are connected. The results are presented in the form of symmetrical components, in percent of the fundamental frequency positive sequence voltage.

Note that a non-characteristic $3 \mathrm{rd}$ harmonic voltage is observable at the compensator bus for all test systems, with magnitudes larger than those of the characteristic harmonics. 
The 5th and 7th harmonic voltage are nearly zero at the compensator bus for the first two examples due to the transformer harmonic cancellation scheme. The 11th and 13th harmonics are present in the second system, because there are no filters to alleviate them. For the last system, more apparent harmonic distortions are observed due to the absence of filters and harmonic cancellations. All test results indicate that the positive and negative sequence harmonic voltages are nearly of the same order of magnitude, even though the fundamental frequency component of the compensator bus voltage has only about $1 \%$ unbalance.
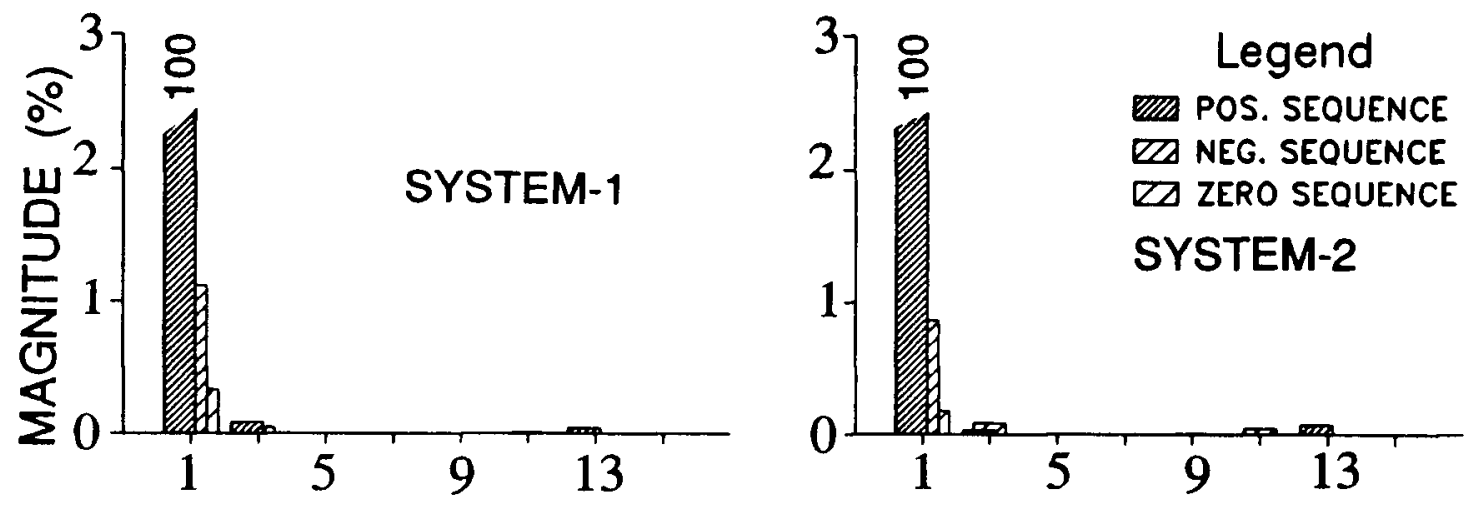

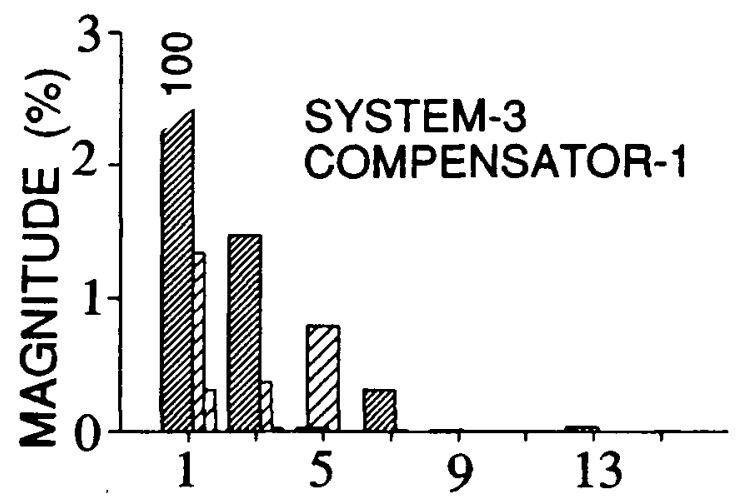

HARMONIC ORDER

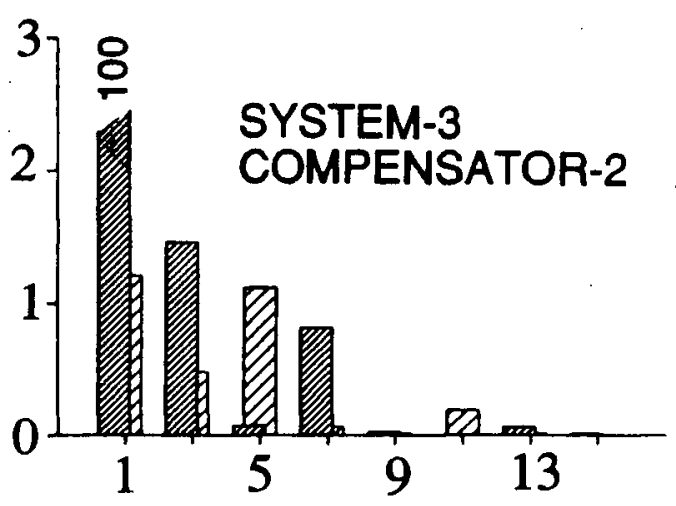

HARMONIC ORDER

Figure 6.8: Static compensator bus voltage harmonic spectrum. 

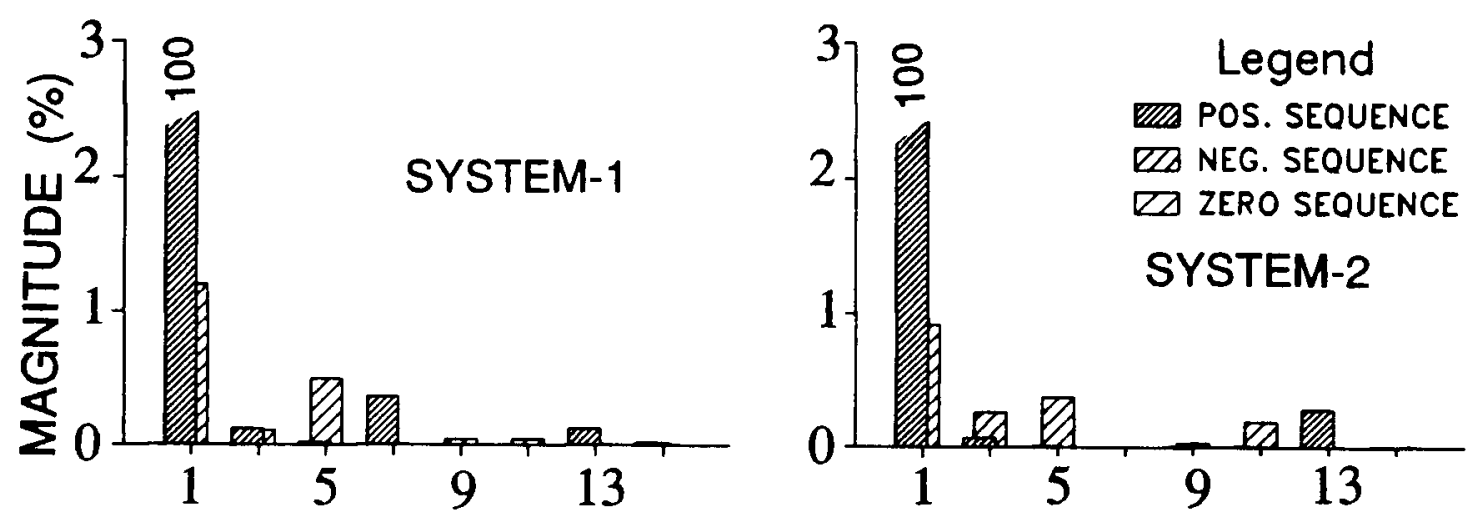

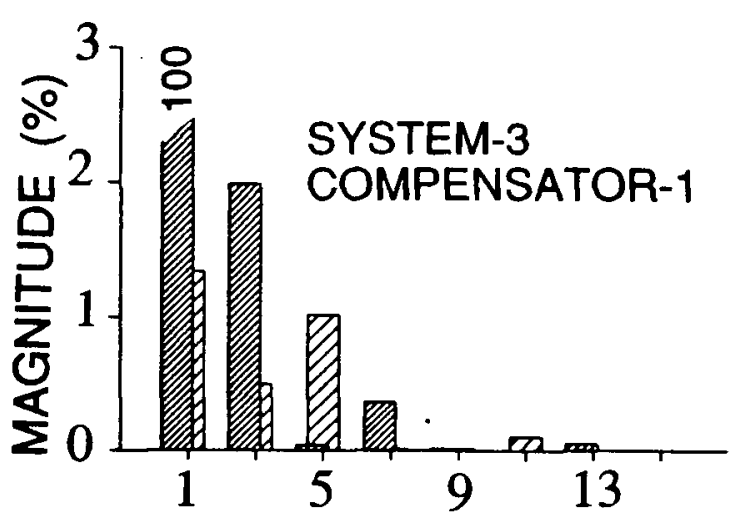

HARMONIC ORDER

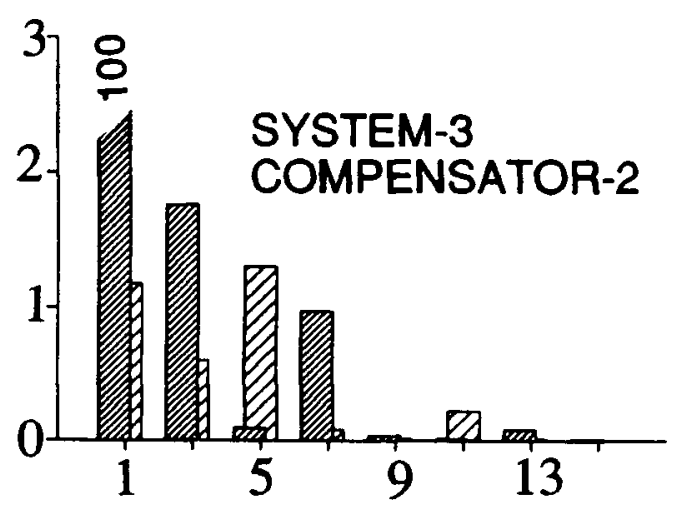

HARMONIC ORDER

Figure 6.9: TCR bus voltage harmonic spectrum.

(2) Current harmonic spectrum.

The harmonic current injections into the network at the compensator bus are shown in Figure 6.10 as positive, negative and zero sequence values in percent based on 100MVA and rated voltage $240 \mathrm{kV}$. The current distortion is found to be larger than that of the voltage. Similar observations have been reported in field measurements. Again, there is a significant amount of non-characteristic harmonic current injections. For all test cases, the harmonic currents are severely unbalanced, with the same order of magnitude in the positive and negative sequence components. 

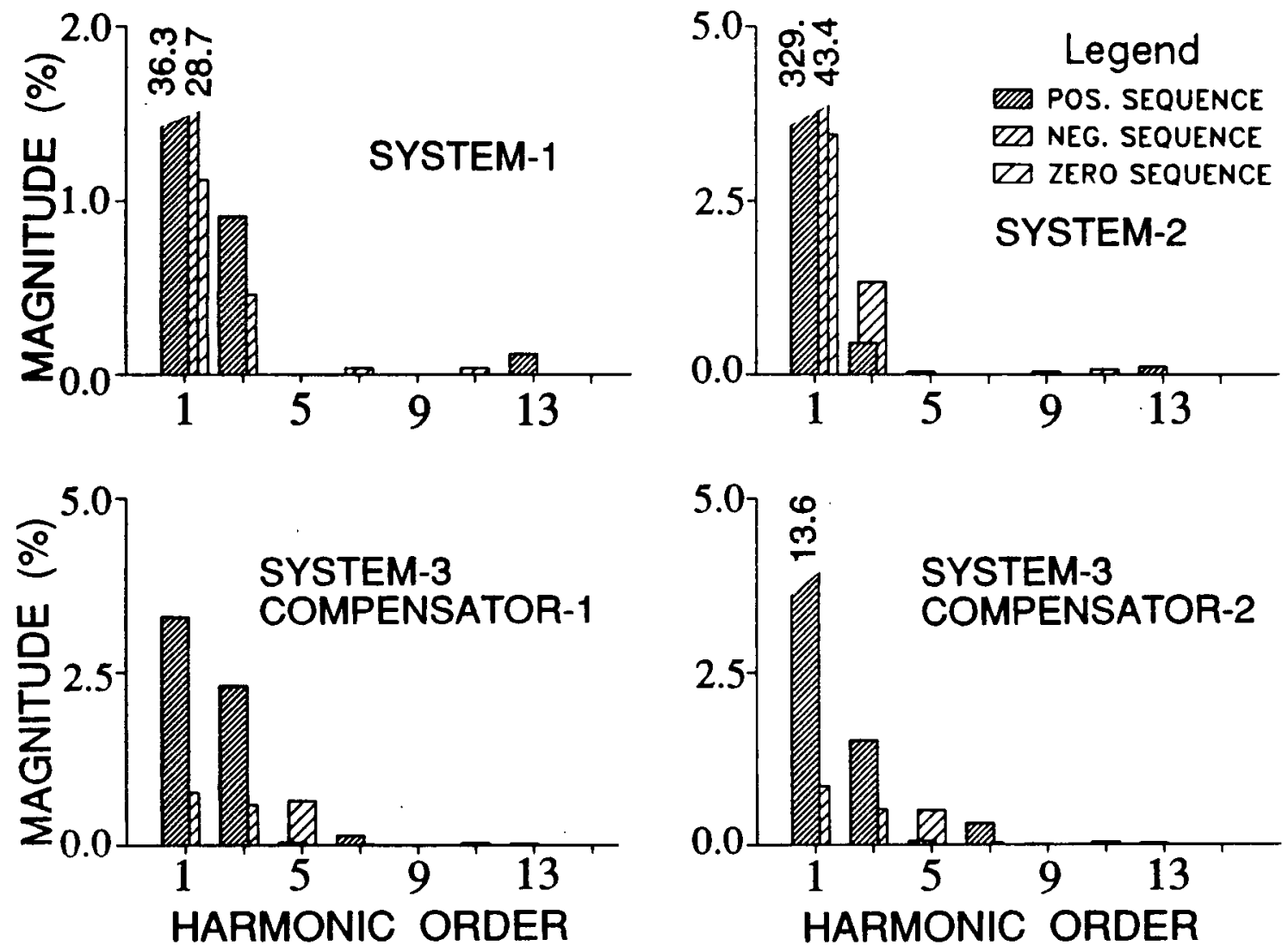

Figure 6.10: Compensator current injection spectrum.

\subsubsection{Convergence Behaviour}

Convergence of the MHLF technique was excellent for all the test cases. Less than 10 iterations were needed to obtain solutions with an accuracy of $10^{-4}$ p.u. The results are summarized in Table 6.1, along with the total computer CPU time.

For these test cases, the TCR conduction angles were automatically adjusted by the program. The convergence characteristics of the adjustment process are plotted in Figure 6.11. The conduction angles converge to the final value in only five iterations. 
Table 6.1: Convergence behaviour of the MHLF technique.

\begin{tabular}{|c|ccc|}
\hline iteration & system-1 & system-2 & system-3 \\
\hline \hline & \multicolumn{3}{|c|}{ maximum update of the TCR } \\
equivalent current & sources \\
\hline 1 & 0.52253 & 0.64732 & 0.06643 \\
2 & 0.01850 & 0.26561 & 0.01906 \\
3 & 0.00637 & 0.04164 & 0.00527 \\
4 & 0.00016 & 0.01893 & 0.01231 \\
5 & 0.00001 & 0.00251 & 0.00506 \\
6 & & 0.00098 & 0.00147 \\
7 & & 0.00027 & 0.00026 \\
8 & & 0.00012 & 0.00011 \\
9 & & 0.00006 & 0.00001 \\
\hline CPU(s) & 308.42 & 96.69 & 265.79 \\
\hline
\end{tabular}

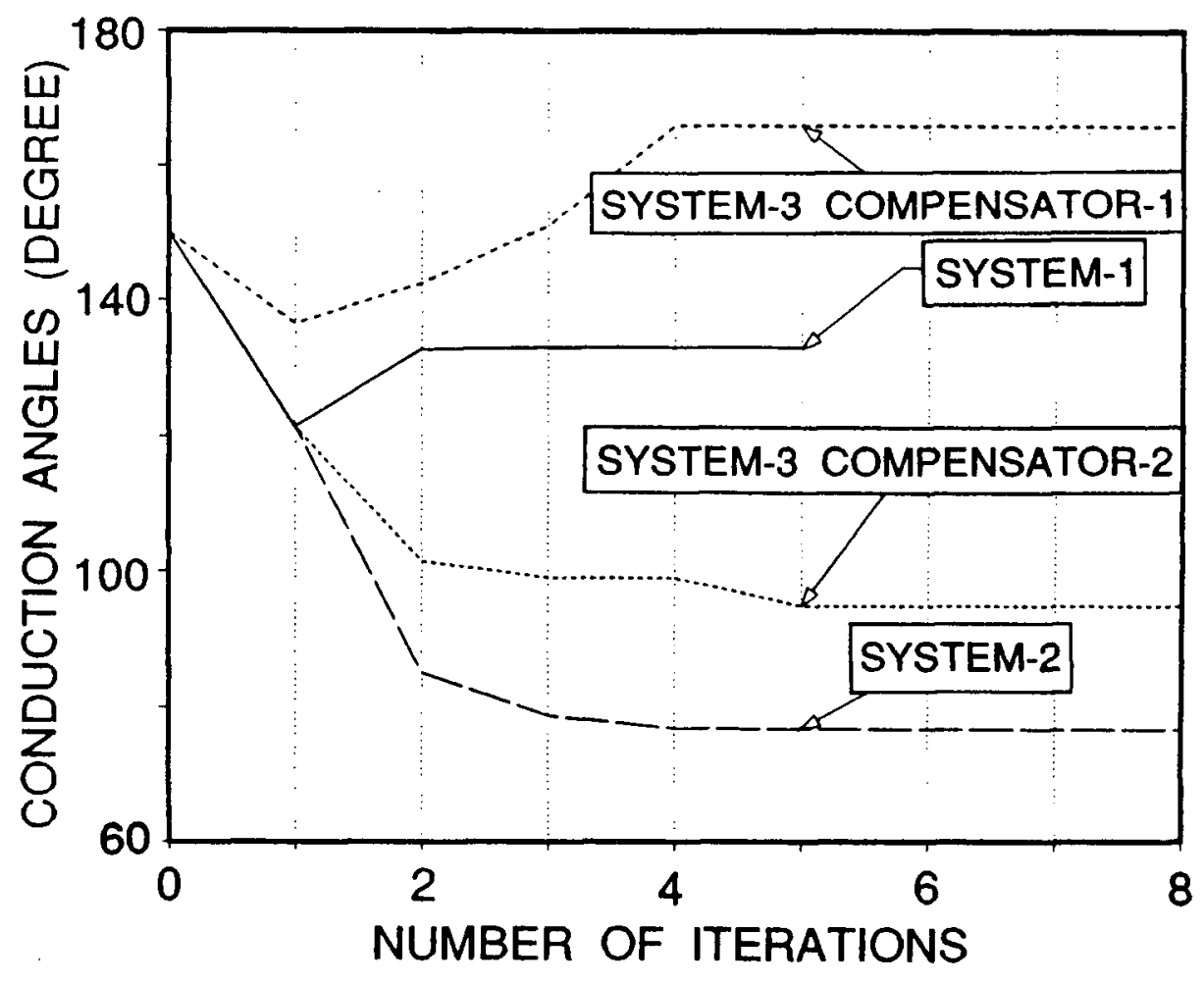

Figure 6.11: Convergence of conduction angles. 
Examination of Table 6.1 and Figure 6.11 suggests that the conduction angles converge first. Once the correct conduction angles are obtained, the harmonic iterative process converges in about 3 to 5 iterations. An initial conduction angle of $150^{\circ}$ was selected in all examples. To speed up the iteration, it is possible to compute an approximate conduction angle from the fundamental frequency component only in an initialization run. This angle could then be used as an initial guess for the harmonic load flow solutions.

\subsection{Summary}

The MHLF technique has been used to analyze the unbalanced harmonic load flows caused by static compensators. It is achieved by first deriving the Norton equivalent circuit of the TCR and then interfacing the model with the MHLF solution process. An efficient conduction angle adjusting scheme has been developed to include the compensator control characteristics. This scheme is very general and simple. It can be used for other nonlinear elements and other control characteristics as well. This chapter illustrated the typical procedure of using MHLF technique for the analysis of nonlinear elements with control characteristics.

The MHLF technique has been tested with a number of static compensator test cases. Good convergence behaviour was observed in all cases. The major observations from the case studies can be summarized as follows:

1. Both the simulation results and the measurements show that there are significant harmonic distortions inside the compensator system. The non-characteristic harmonics caused by unbalanced operation can also be significant in some cases.

2. The non-characteristic harmonic current injections are generally proportional to the degree of unbalance in the compensator bus voltage, TCR reactances, and firing angles. 
3. The harmonic components are more sensitive to unbalanced conditions than the fundamental frequency components.

4. The waveform distortions in the network are low in properly designed static compensator systems. In particular, the harmonic cancellation scheme with a threewinding transformer connection is quite effective in most unbalanced cases. 


\section{Chapter 7}

\section{Convergence Analysis of the MHLF Technique}

\subsection{General Considerations}

The MHLF techniques presented in previous chapters are based on the harmonic iteration scheme. With this scheme, the nonlinear effects are represented as harmonic current sources and then solved with the network equations, which makes the frequency-domain steady-state analysis of the network possible. However, this advantage is achieved with the cost of iteration. Although various case studies have exhibited the good convergence behaviour of the iteration, they are still not enough to explain the mechanism of the convergence and to identify possible causes if divergence happens.

A more rigorous analysis of the MHLF convergence behaviour is performed in this chapter. It provides a unified view on the iteration solutions of three apparently different nonlinearities studied in this work. Factors that affect convergence performance are identified. Convergence criteria are developed for some simplified cases. These criteria are also useful to explain the convergence of the general cases.

The convergence analysis is divided into two steps. In the first step, cases of static networks are analyzed, which makes it possible to visualize the iteration process. In the second step, the general cases are discussed. 


\subsection{A Simple Case: Static Network}

One of the main difficulties in the MHLF convergence analysis is that the results of each iteration are functions of time. This time-dependent iteration process is hard to visualize. To eliminate this difficulty, the analysis of the harmonic iteration in static networks is first considered. An example of such networks is a resistive network with nonlinear resistors. Since there are no dynamics in such networks, the network voltages and currents can be determined according to the instantaneous values of the sources and the network characteristics. Thus the explicit time-dependent solutions are then avoided.

\subsubsection{A Convergence Theorem}

Starting with the simplest case, the solution of the following equation is first considered:

$$
\left\{\begin{array}{l}
y=f(x) \\
x=g(y)
\end{array}\right.
$$

where $x$ and $y$ are simple real variables. The equations can be solved by the following iteration scheme

$$
y_{i}=f\left(x_{i}\right), \quad x_{i+1}=g\left(y_{i}\right),
$$

where $i$ is the number of iterations. A convergence theorem is developed for this case.

Theorem 1. Let $\Omega_{x}=\left[\begin{array}{ll}a, b\end{array}\right], \Omega_{y}=\left[\begin{array}{ll}c, d\end{array}\right]$ be closed finite intervals, and let the continuous functions $y=f(x), x=g(y)$ satisfy the following conditions:

1. $f(x) \in \Omega_{y}$ for all $x \in \Omega_{x} ;$ and $g(y) \in \Omega_{x}$ for all $y \in \Omega_{y}$.

2. $\left|f^{\prime}(x)\right| \leq c_{1}$, for all $x \in \Omega_{x} ; \quad\left|g^{\prime}(y)\right| \leq c_{2}$, for all $y \in \Omega_{y} ; \quad$ and $\quad c=c_{1} c_{2}<1$.

Then

1. There is a unique point $\alpha \in \Omega_{x}, \beta \in \Omega_{y}$ such that $\beta=f(\alpha), \alpha=g(\beta)$. 
2. For any choice of $x_{o} \in \Omega_{x}$, the sequence defined by iteration (7.2) converges to the point.

3. The rate of convergence is related to the parameter $c$ by the following equation

$$
\left|x_{i}-\alpha\right| \leq \frac{c^{i}}{1-c}\left|x_{1}-x_{o}\right|
$$

The proof of this theorem is given in Appendix B. The iteration process is shown in Figure 7.1. As shown in this figure, the basic requirement for the process to converge is a proper angle between the tangent lines of the two curves. This condition is formally stated as $c<1$ in the theorem.

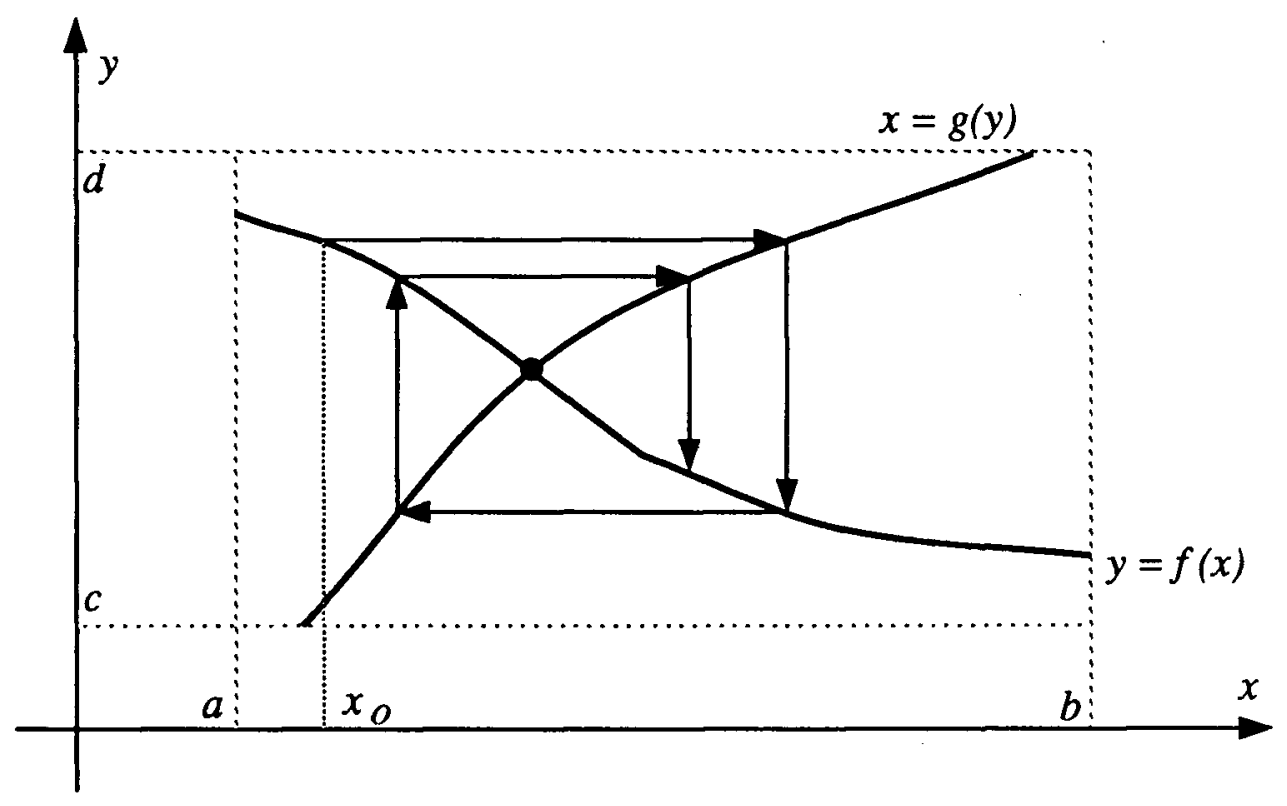

Figure 7.1: Iterative solution process of Equation (7.1). 


\subsubsection{Analysis of the Basic Iterative Scheme}

The application of Theorem 1 is best illustrated by analyzing the harmonic solution of the simple resistive network shown in Figure 7.2(a). Assuming that the characteristics of the nonlinear resistor are described as $i=g(v)$ (Figure 7.2(b)), the network equation is

$$
v(t)=e(t)-R i(t), \quad i(t)=g(v(t)),
$$

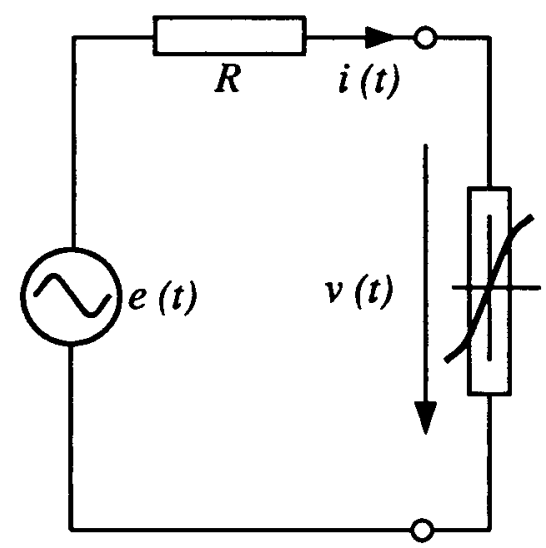

(a) network diagram

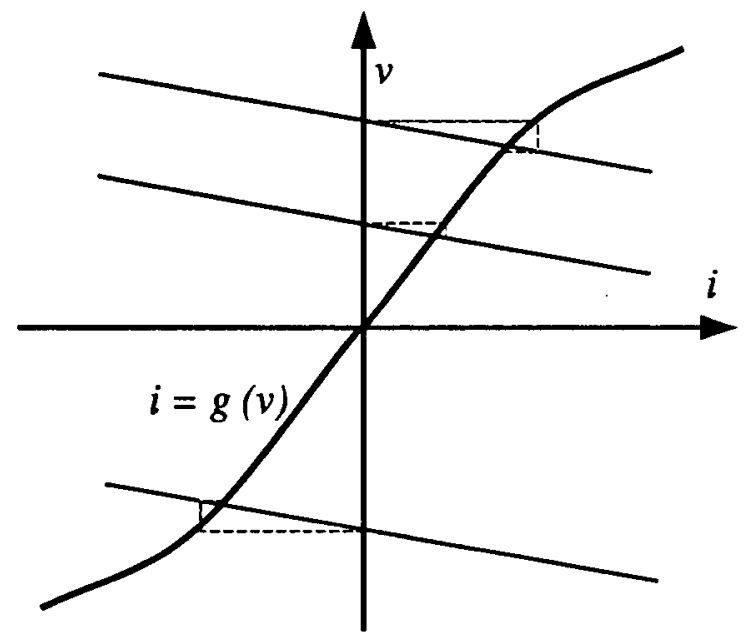

(b) iteration process

Figure 7.2: Harmonic iteration of a simple resistive network.

where $e(t)$ is a harmonic voltage source. Since this is a static network, the solution can be determined point-by-point from the instantaneous source voltage value. The circuit equations can therefore be rewritten as

$$
v=e-R i, \quad i=g(v) .
$$

The harmonic iteration scheme of this network corresponds exactly therefore to the case 
described in Theorem 1. Applying Theorem 1 to the equation yields

$$
c_{1}=R, \quad c_{2}=g^{\prime}(v)_{\max }
$$

Since $g^{\prime}(v)$ is a monotonic increasing function and the iteration starts with $i(t)=0$, $g^{\prime}(v)_{\max }$ can be selected as

$$
g^{\prime}(v)_{\max }=g^{\prime}\left(e_{m}\right)
$$

where $e_{m}$ is the peak value of the source voltage. The convergence condition is then obtained as

$$
c=c_{1} c_{2}=R g^{\prime}\left(\epsilon_{m}\right)<1
$$

This condition is intuitively obvious from Figure 7.2(b). Further analysis leads to following useful conclusions:

1. As shown in Eq. (7.3), the increase of the peak source voltage causes the increase of constant $c$. According to the Theorem, the rate of convergence is then reduced. This situation implies 'deep saturation' of nonlinear resistors or inductors in practice, hence confirms the observations that the degrees of saturation have adverse effects on the rate of convergence.

2. If $R$ increases, $c$ also increases. This case can also result in poor convergence rate or even divergence. The practical implication of the situation is the voltage harmonic resonance, where the magnitude of the equivalent network impedance is large. The conclusion is that the harmonic voltage resonance is likely to cause convergence difficulties.

3. Instead of using the current iteration, the following voltage iteration may be considered

$$
i=(e-v) / R, \quad v=q(i),
$$


where $q$ is the inverse function of $g$. According to Theorem 1 , the convergence criterion is

$$
c_{v-\text { iteration }}=(1 / R)\left|q^{\prime}(i)\right|_{\max }=(1 / R)\left|\underline{1} / g^{\prime}(v)\right|_{\max }=\left(R g^{\prime}(0)\right)^{-1}<1 .
$$

Note that $c_{v-\text { iteration }}$ is related to $g^{\prime}(0)$, and therefore is not the inverse of the parameter $c$ in the current iteration (Eq. (7.3)). This suggests that depending on the scheme used the convergence difficulties can happen at different regions of the $g(v)$ curve. For the same reason, 'deep saturation' is no longer a problem in the voltage iteration, which is an advantage of the scheme ${ }^{1}$.

4. The advantage of the Norton equivalent circuit over the simple current source modelling techniques can be formally justified by comparing the parameters $c$ 's for the two models. For the Norton equivalent circuit approach, the network equation can be written as:

$$
v=e_{n}-\left(R / / R_{e}\right) i, \quad i=g(v)-v / R_{e},
$$

where $R_{e}=1 / g^{\prime}(o)$, the equivalent resistance of the nonlinear element. $e_{n}$ is the new source voltage. The new parameter $c$ is

$$
c_{\text {notion }}=\left(R / / R_{e}\right)\left(g^{\prime}\left(e_{m}\right)-1 / R_{e}\right) .
$$

Since $g^{\prime}(v)>0$ and is a increasing function, and $R / / R_{e}<R$, we have

$$
\begin{aligned}
c_{\text {norton }} & =\left(R / / R_{e}\right)\left(g^{\prime}\left(e_{m}\right)-1 / R_{e}\right) \\
& <R\left(g^{\prime}\left(e_{m}\right)-1 / R_{e}\right) \\
& <R g^{\prime}\left(e_{m}\right)=c_{\text {current }}
\end{aligned}
$$

According to the 4th conclusion of the theorem, the convergence is improved. This improvement can be clearly seen from Figure 7.3.

\footnotetext{
${ }^{1}$ Care shall be taken to use this scheme since $g^{\prime}(0)$, the inverse of unsaturated resistance, can be very small. This may also lead to $c_{v-i t e r a t i o n}>1$.
} 
It shall be noted that the discussion is not only limited to the resistive networks. It is also suitable to the nonlinear inductor connected to a network with very small resistors and capacitors (inductive network).

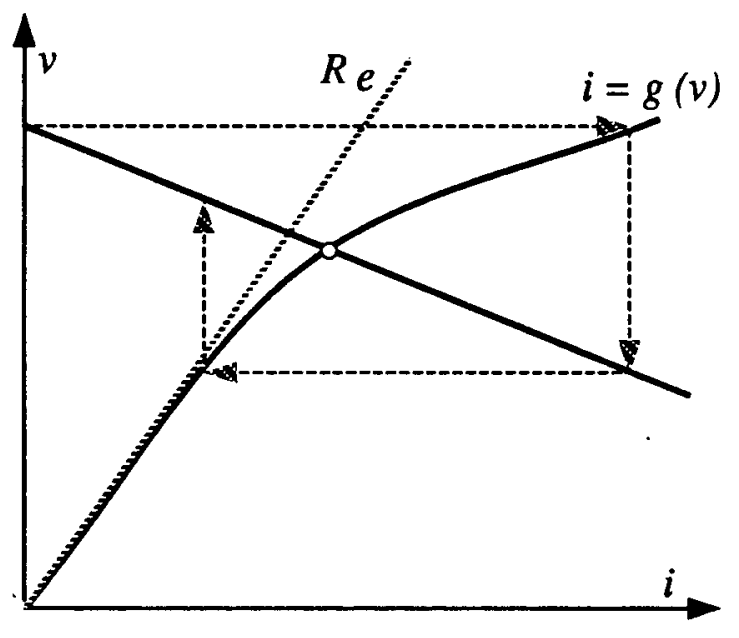

(a) current source model

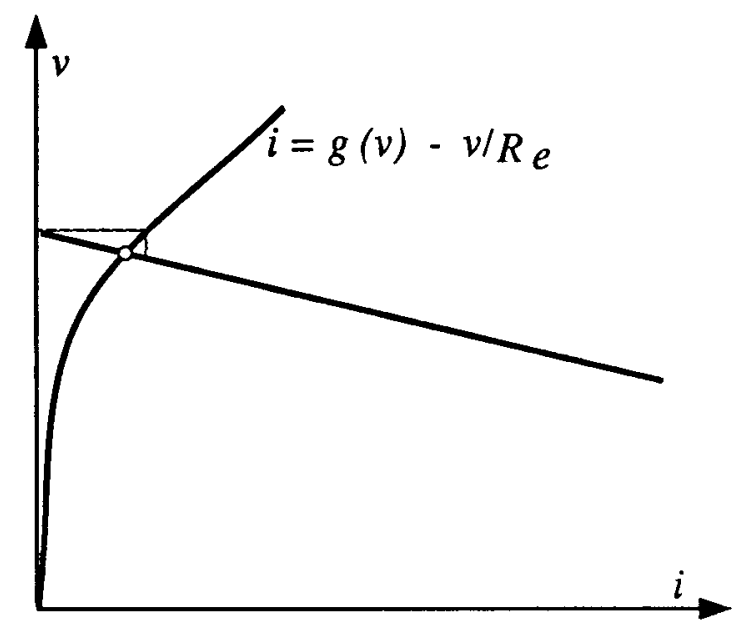

(b) Norton equivalent model

Figure 7.3: Convergence comparison between two modelling techniques.

\subsubsection{Cases with More Than One Nonlinear Elements}

For the cases with more than one nonlinear elements, the network equations can always be simplified as

$$
[v]=[e]-[R][i], \quad[i]=g([v]) .
$$

Again the notation $(t)$ is dropped. To simplify the problem, it is assumed that

$$
[i]=g([v])=\left[g_{1}\left(v_{1}\right), \ldots, g_{k}\left(v_{k}\right), \ldots, g_{m}\left(v_{m}\right)\right]^{T}
$$

where $\mathrm{m}$ is the number of nonlinear elements, and $g_{k}$ is continuous. 
Direct visualization of the iteration process is no longer possible for such a network. Similar convergence conditions can be developed however. These conditions are presented in the following theorem.

Theorem 2: Assume that Eq. (7.4) has a solution $[i]=[\alpha],[v]=[\beta]$, and that

$$
\left\|g\left(\left[v_{a}\right]\right)-g\left(\left[v_{b}\right]\right)\right\| \leq c_{2}\left\|\left[v_{a}\right]-\left[v_{b}\right]\right\|, \quad \text { where } \quad\|[v]-[\beta]\| \leq \xi
$$

Let $c_{1}=\|[R]\|$. If $c=c_{1} c_{2}<1$, then the iteration

$$
\left[v_{i}\right]=[e]-[R]\left[i_{i}\right], \quad\left[i_{i+1}\right]=g\left(\left[v_{i}\right]\right)
$$

started with $\left[i_{o}\right]$, where $\left\|[e]-[R]\left[i_{o}\right]-[\beta]\right\| \leq \xi$, converges to the solution point.

The proof is also given in the Appendix B. To make use of the theorem, parameters $c_{1}$ and $c_{2}$ must be related to the network conditions. According to matrix theory [40], a norm of $[R]$ can be defined as:

$$
c_{1}=\|[R]\|_{\infty}=\operatorname{Max}_{i} \sum_{j=1}^{m}\left|R_{i j}\right| .
$$

For function $g([v])$, it is true for each component

$$
\left|g_{k}\left(v_{a}\right)-g_{k}\left(v_{b}\right)\right| \leq\left|g_{k}^{\prime}(v)\right|_{\max }\left|v_{a}-v_{b}\right|
$$

Therefore the norm of $g([v])$ can be selected as the maximum derivatives of its components, namely,

$$
c_{2}=\operatorname{Max}_{k=1, m}\left|g_{k}^{\prime}(v)\right|,
$$

where voltage $v$ takes values in the $\xi$ region of $[\beta]$.

\subsection{General Case: Dynamic Network}

In this section, the convergence conditions are extended to nonlinear dynamic networks. In such networks, harmonic solutions must be considered as time functions in the entire 
iteration process. With the MHLF technique as a major concern, the harmonic iteration concept can be more rigorously stated as the solution of the following two transformations:

$$
\left\{\begin{array}{l}
{[v(t)]=T_{1}\{[i(t)]\}} \\
{[i(t)]=T_{2}\{[v(t)]\},}
\end{array}\right.
$$

where $[v(t)]$ denotes harmonic voltages at the terminals of nonlinear elements, and $[i(t)]$ the harmonic current injections of those elements. Transformation $T_{1}$ represents the network solution process (harmonic frequency scan plus load flow solutions at the fundamental frequency), while $T_{2}$ represents the computation of Norton or current source equivalent circuits for the nonlinear elements. The iteration process can be described as the successive transformation of harmonic waveforms in the harmonic space:

$$
\left[v_{i}\right]=T_{1}\left(\left[i_{i}\right]\right), \quad\left[i_{i+1}\right]=T_{2}\left(\left[v_{i}\right]\right)
$$

where $(t)$ is dropped to simplify the notations. To analyze the convergence property of this process, the theory of numerical functional analysis must be employed [41].

\subsubsection{Contraction Mappings in Harmonic Space}

Based on the theory of numerical functional analysis [41], following theorem is developed.

Theorem 3: Let $T_{1}: X \rightarrow Y$ be a transformation from Banach spaces $X$ to $Y$, and $T_{2}: Y \rightarrow X$ a transformation from $Y$ to $X$. If

1. $T_{1}$ is bounded in subspace $X_{s} \in X:\left\|T_{1}\left(\left[i_{a}\right]\right)-T_{1}\left(\left[i_{b}\right]\right)\right\| \leq c_{1}\left\|\left[i_{a}\right]-\left[i_{b}\right]\right\|$.

2. $T_{2}$ is bounded in subspace $Y_{s} \in Y:\left\|T_{2}\left(\left[v_{a}\right]\right)-T_{2}\left(\left[v_{b}\right]\right)\right\| \leq c_{2}\left\|\left[v_{a}\right]-\left[v_{b}\right]\right\|$.

3. $T_{1}([i]) \in Y_{s}$ for all $[i] \in X_{s}$, and $T_{2}([v]) \in X_{s}$ for all $[v] \in Y_{s}$

4. $c=c_{1} c_{2}<1$. 
Then for $\left[i_{o}\right] \in X_{s}$, the sequence $\left\{\left[i_{i}\right]\right\}$ defined by iteration $(7.6)$ has a limit. This limit is unique and equal to the solution point $[\alpha]:[\beta]=T_{1}([\alpha]),[\alpha]=T_{2}([\beta])$.

The proof is given in Appendix B. This Theorem can directly be used to analyze the harmonic iteration scheme. The following remarks are observed for this purpose:

1. For harmonic analysis, space $X$ denotes the collection of all harmonic current functions, and $Y$ the collection of all harmonic voltage functions. The requirement that $X$ and $Y$ are Banach spaces are naturally satisfied, since $X$ and $Y$ represent continuous harmonic functions.

2. The combined transformation, $T_{2} T_{1}: X \rightarrow X$, that satisfies the conditions of Theorem 3 is called contraction mapping [41]. With this transformation, for each input, the output stays in the same space as that of input, but the distance between the output and the solution point is reduced by a factor c, compared with the distance between the input and the solution. With repeated application of the transformation, any initial input will gradually be 'attracted' to the solution point. This solution point, which is the center of the attraction, is therefore also called fixed point. The contraction process can roughly be visualized by referring to Figure 7.1. In this case, axes $x$ and $y$ shall be imagined as harmonic function spaces $X$ and $Y$.

3. The importance of selecting a proper starting point is emphasized through the requirement of subspaces $X_{a}$ and $Y_{B}$, which describe the region where the contraction takes place. For the purpose of power system harmonic analysis, harmonic components are generally small and initialization with fundamental frequency load flow results was found appropriate. 
4. In the MHLF analysis, transformation $T_{1}$ represents the decoupled harmonic frequency scan. Therefore, it can be further expressed as

$$
T_{1}([i])=T_{1}\left(\sum_{h=1}^{n}[i(h)]\right)=\sum_{h=1}^{n} T_{1-h}([\mathbf{I}(h)]),
$$

where $h$ is the harmonic order, and $[\mathbf{I}(h)]$ is the current vector $[i]$ expressed in the form of harmonic phasors. All of the $T_{1-h}$ transformations are linear except that of $h=1$ which represents load flow solutions. Assuming there are no PQ and PV constraints, $T_{1-1}$ also becomes a linear operation. Thus, according to the Norton equivalent network theory, the network solution can always be simplified as:

$$
T_{1-h}([\mathbf{I}(h)])=[\mathbf{E}(h)]-[Z(h)][\mathbf{I}(h)],
$$

where $[\mathbf{E}(h)]$ is the equivalent source voltage vector and $[Z(h)]$ equivalent network impedance matrix. Since

$$
\left\|T_{1-h}\left(\left[I_{a}(h)\right]\right)-T_{1-h}\left(\left[I_{b}(h)\right]\right)\right\| \leq \|\left[Z(h)\|\|\left[I_{a}(h)\right]-\left[I_{b}(h)\right] \|,\right.
$$

it can be seen that parameter $c_{1}$ is directly related to the norm of the impedance matrices. Similar conclusions can also be valid even $\mathrm{PQ}$ and $\mathrm{PV}$ constraints are imposed, because the constraints only affect the $T_{1-1}$ transformation, and this transformation can be linearized around the solution point.

5. $T_{2}$ transformation is a nonlinear one. Its bounding parameter $c_{2}$ is entirely determined by the nonlinearity under consideration. No general comments are available. However some preliminary results obtained for the typical nonlinearities studied in this dissertation are explained below.

\subsubsection{Application 1: Nonlinear Inductor and Harmonic Resonance}

In this subsection, Theorem 3 is used to analyze the simple nonlinear inductor circuit shown in Figure 7.4. The circuit equation is 


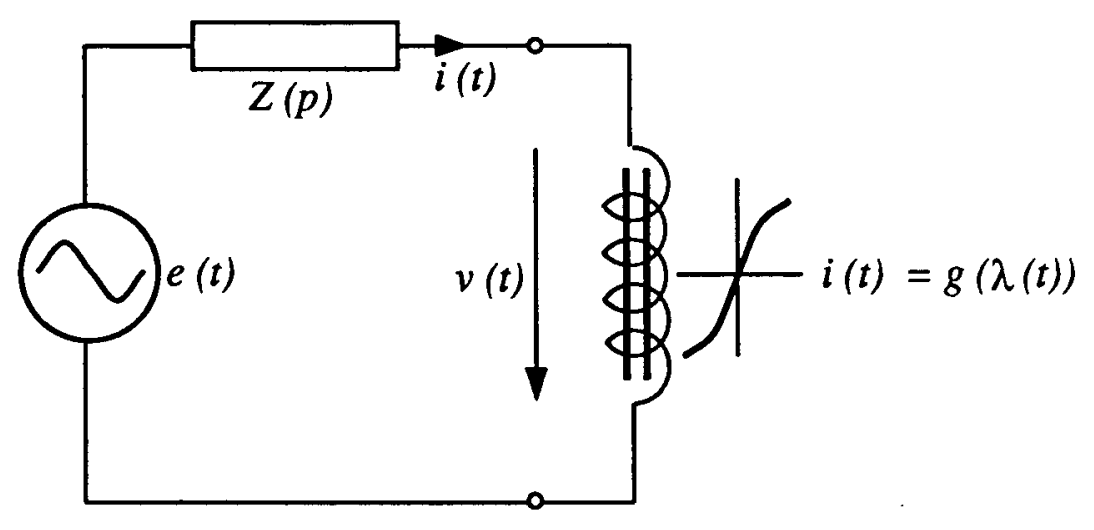

Figure 7.4: A simple nonlinear inductor circuit.

$$
v(t)=e(t)-Z(p) i(t), \quad i(t)=g(\lambda(t)),
$$

where $p=\frac{d}{d t}$ and $v(t)=p \lambda(t)$. Using $\lambda$ as a variable and simplifying the equation above yields:

$$
\begin{array}{cc}
T_{1}: & \lambda(t)=\frac{e(t)}{p}-\frac{Z(p)}{p} i(t), \\
T_{2}: & i(t)=g(\lambda(t)) .
\end{array}
$$

This form is suitable for the application of Theorem 3. For the first transformation,

$$
\left\|\lambda_{a}(t)-\lambda_{b}(t)\right\|=\left\|\frac{Z(p)}{p}\left(i_{a}(t)-i_{b}(t)\right)\right\| \leq\left\|\frac{Z(p)}{p}\right\|\left\|\left(i_{a}(t)-i_{b}(t)\right)\right\| .
$$

Since $\frac{Z(p)}{p}$ is a single variable transformation and only the harmonic components are involved in the transformation

$$
\left\|\frac{Z(p)}{p}\right\|_{2} \leq M a x_{h=1, n}\left|\frac{Z(j h \omega)}{(j h \omega)}\right|=c_{1}
$$


where $n$ is the maximum harmonic order considered. For transformation $T_{2}$,

$$
\left\|i_{a}(t)-i_{b}(t)\right\|=\left\|g\left(\lambda_{a}(t)\right)-g\left(\lambda_{b}(t)\right)\right\| \leq \mid g^{\prime}[\xi(t)]\|\| \lambda_{a}(t)-\lambda_{b}(t) \|,
$$

where

$$
\left[\lambda_{a}(t), \quad \lambda_{b}(t)\right]_{\min } \leq \xi(t) \leq\left[\lambda_{a}(t), \quad \lambda_{b}(t)\right]_{\max } .
$$

Since $g^{\prime}(\lambda)$ is a monotonic function for $\lambda>0$, we can let

$$
c_{2}=\left|g^{\prime}\left(\lambda_{\max }\right)\right|
$$

where $\lambda_{\max }$ is the possible maximum flux experienced by the inductor. The contraction factor is therefore:

$$
c=c_{1} c_{2}=g^{\prime}\left(\lambda_{\max }\right)\left|\frac{Z(j h w)}{h w}\right|_{\max } .
$$

The sufficient condition for convergence is $c<1$. As explained in last section, the parameter $c$ also determines the rate of convergence. Further analysis of the convergence condition suggests the following:

1. Harmonic voltage resonance indicates a large increase in $|Z(j h w)|$. Under such condition, the contraction factor $c$ will be increased. Therefore the harmonic voltage resonance is indeed a major factor of convergence difficulties.

2. It is interesting to note that $c$ is inversely related to the harmonic order. For the same $|Z(j h \omega)|$, low-order harmonics are more influential than the high-order harmonics on the rate of convergence. The reason behind this observation is the low pass filtering effect of all inductors.

3. Deep saturation implies an increase of $g^{\prime}\left(\lambda_{\max }\right)$, which also increases the contraction factor c. Slower convergence is expected in this situation. If the $\lambda-i$ curve is represented by a piecewise linear function, the convergence rate is then related to the segment of the curve with the least incremental inductance. 
4. The number of harmonics included in the iteration has no direct adverse effects on the convergence.

5. For this specific case, the selection of starting point is related to $\lambda_{\max }$. As long as the initial $\lambda_{\max }$ holds the condition $c<1$, the convergence is guaranteed.

This convergence condition can also be used to analyze the convergence difference between Norton equivalent circuit and current source modelling of the nonlinear inductors. Results indicate that convergence improvement can be obtained but it depends on $Z(j h \omega)$. All of these conclusions are consistent with the results obtained in the previous section. They also demonstrate the effectiveness of static network approach.

\subsubsection{Application 2: Thyristor-controlled Reactors}

To analyze the convergence behaviour of the harmonic iteration scheme for TCR's, only the second transformation $[i]=T_{2}([v])$ needs to be considered. Using switch function theory, it can be shown that the bounding parameter $c_{2}$ for the TCR transformation is $L^{-1}$, where $L$ is the TCR inductance. Instead of following this approach, an alternative idea is presented, which relates the TCR characteristics with that of nonlinear inductors.

The TCR characteristics can be approximately described as a $\lambda-i$ curve, as shown in Figure 7.5. The parameter $\lambda_{0}$ in the figure is determined by the conduction angle and the terminal voltage magnitude. It can be seen that this characteristic gives the same current waveform as that of the real TCR. Although this $\lambda-i$ model is approximate and is only suitable for steady-state analysis, it is sufficient for the convergence analysis.

With this model, the TCR can simply be considered as one type of nonlinear inductor. According to the analysis of the last subsection, the bounding parameter $c_{2}$ is $L^{-1}$ which is the same as the one from switch function theory. Most of the conclusions drawn for the nonlinear inductors are also suitable for TCR's. In addition the following are observed: 


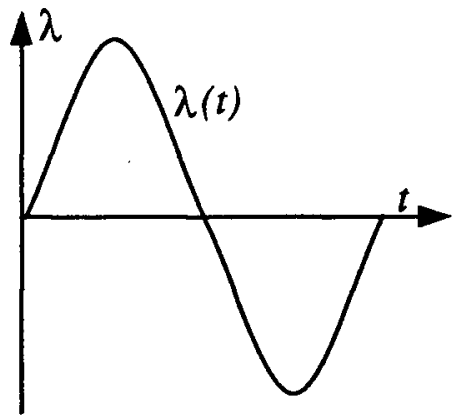

(a) $\lambda$ waveform

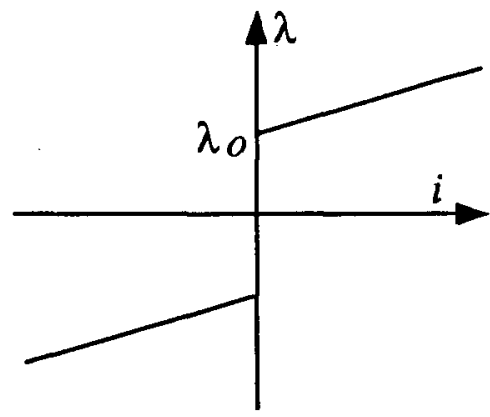

(b) $\lambda-i$ curve

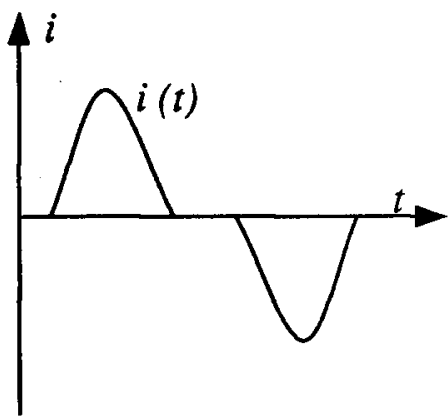

(c) $i$ waveform

Figure 7.5: TCR $\lambda-i$ characteristics.

1. The convergence rate is independent of the supply voltages, i.e. there is no 'deep saturation' in the TCR case.

2. The convergence rate is inversely related to the TCR inductance. One typical example of this observation is the case with $L \rightarrow 0$. In this case, an infinite TCR current is injected into the network, which will certainly cause numerical divergence.

\subsubsection{Application 3: Synchronous Machines}

The iteration scheme for solving the synchronous machine harmonics is unique due to its harmonic conversion mechanism. Under the framework of contraction operations, it is found that the scheme has many similarities with the iterative processes of other nonlinear elements. In this subsection, the convergence conditions for synchronous machine iteration are developed under certain operating conditions.

Assume that there are no $P Q$ and $P V$ constraints and that there is only one nonlinear machine in the network. With reference to Figure 7.6, the network equation can be written as: 


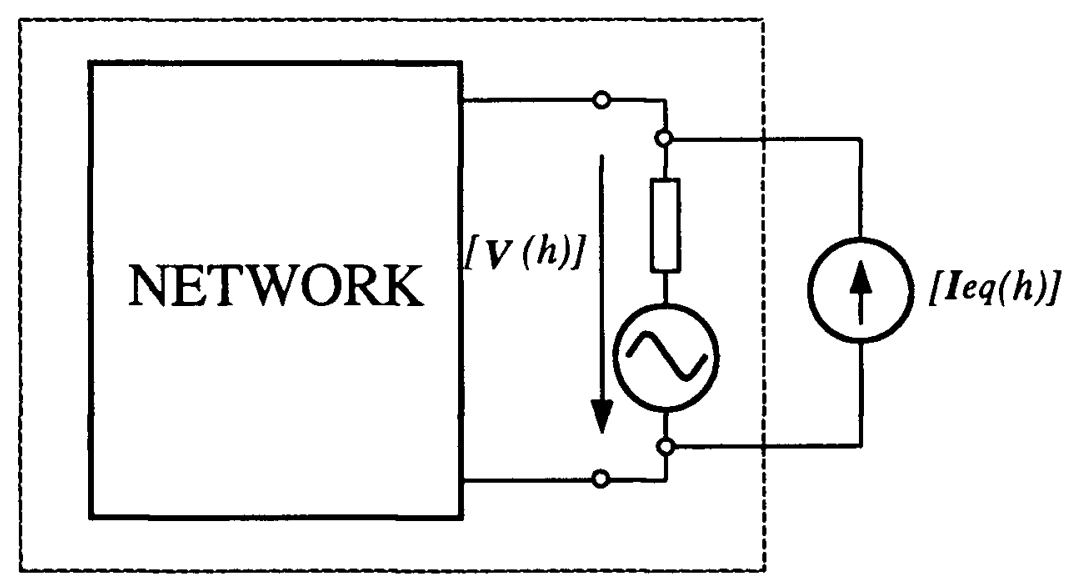

Figure 7.6: A synchronous machine connected to a network (single-line diagram).

$$
[\mathbf{V}(h)]=[\mathbf{E}(h)]-[Z(h)]\left[\mathbf{I}_{e q}(h)\right], \quad h=1, \ldots, n,
$$

where $\left[\mathbf{I}_{e q}(h)\right]$ is the equivalent current injection to account for the machine nonlinearity. These equations can be further expressed in a combined form, as follows:

$$
\left[\begin{array}{l}
\mathbf{V}(1) \\
\mathbf{V}(3) \\
\cdots \\
\mathbf{V}(n)
\end{array}\right]=\left[\begin{array}{l}
\mathbf{E}(1) \\
0 \\
\cdots \\
0
\end{array}\right]-\left[\begin{array}{llll}
Z(1) & 0 & \ldots & 0 \\
0 & Z(3) & \ldots & 0 \\
\ldots & \ldots & \ldots & \ldots \\
0 & 0 & \ldots & Z(n)
\end{array}\right]\left[\begin{array}{l}
\mathbf{I}_{e q}(1) \\
\mathbf{I}_{e q}(3) \\
\ldots \\
\mathbf{I}_{e q}(n)
\end{array}\right]
$$

or

$$
\left[\mathbf{V}_{H}\right]=\left[\mathbf{E}_{H}\right]-\left[Z_{H}\right]\left[\mathbf{I}_{H-e q}\right]
$$

If only frequency conversion is considered, the equivalent harmonic current source $\left[\mathrm{I}_{e q}(h)\right]$ is related to voltage $[\mathbf{V}(h)]$ in the form of Eq. (5.9) of Chapter 5, or 


$$
\left[\begin{array}{l}
\mathbf{I}_{e q}(1) \\
\mathbf{I}_{e q}(3) \\
\ldots \\
\mathbf{I}_{e q}(n)
\end{array}\right]=\left[\begin{array}{l}
\mathbf{I}_{f}(1) \\
\mathbf{I}_{f}(3) \\
\ldots \\
\mathbf{I}_{f}(n)
\end{array}\right]=\left[\begin{array}{llll}
0 & D^{H} Y_{d q o}(2) D^{c} & \ldots & 0 \\
D^{T} Y_{d q o}(2) D & 0 & \ldots & 0 \\
\ldots & \ldots & \ldots & \ldots \\
0 & 0 & \ldots & 0
\end{array}\right]\left[\begin{array}{c}
\mathrm{V}(1) \\
\mathrm{V}(3) \\
\ldots \\
\mathrm{V}(n)
\end{array}\right]
$$

The compact form is

$$
\left[\mathbf{I}_{H-e q}\right]=\left[Y_{f}\right]\left[\mathbf{V}_{H}\right]
$$

So that the iteration process is:

$$
\begin{array}{lc}
T_{1}: & {\left[\mathrm{V}_{H}\right]_{i}=\left[\mathbf{E}_{H}\right]-\left[Z_{H}\right]\left[\mathbf{I}_{H-e q}\right]_{i},} \\
T_{2}: & {\left[\mathbf{I}_{H-e q}\right]_{i+1}=\left[Y_{f}\right]\left[\mathbf{V}_{H}\right]_{i} .}
\end{array}
$$

The contraction factor of such a linear transformation is simply $c=\left\|\left[Z_{H}\right]\right\|\left\|\left[Y_{f}\right]\right\|$. Since both matrices are square matrices, the convergence condition can be further related to the eigenvalues of the matrices [40]:

$$
c=\left\|\left[Z_{H}\right]\right\|_{2}\left\|\left[Y_{f}\right]\right\|_{2}=\Lambda_{\max }\left(\left[Z_{H}\right]\right) \Lambda_{\max }\left(\left[Y_{f}\right]\right)<1,
$$

where function $\Lambda_{\max }($.$) denotes the magnitude of the largest eigenvalue of the input$ matrix. Since both $\left[Z_{H}\right]$ and $\left[Y_{H}\right]$ are independent of the load flow results, the convergence analysis can easily be performed.

This SM iteration process can also be looked at from the point of view of control theory. Combining transformations $T_{1}$ and $T_{2}$, the following discrete system is established:

$$
\left[\mathbf{I}_{H-e q}\right]_{i+1}=-\left[Y_{f}\right]\left[Z_{H}\right]\left[\mathbf{I}_{H-e q}\right]_{i}+\left[Y_{f}\right]\left[\mathbf{E}_{H}\right]
$$

The condition for this system to be stable is that all eigenvalues of the state matrix $\left[Y_{f}\right]\left[Z_{H}\right]$ are inside the unit circle. This condition is consistent with that required by the convergence condition of Eq. (7.7). 


\subsection{Summary}

A systematic convergence analysis of the MHLF technique has been presented. With this study, it is found that the combination of two parameters determines the convergence behaviour of the technique. The first parameter $c_{1}$ is related to the voltage response of a network when harmonic currents are injected, and the second parameter $c_{2}$ is related to the current response of the nonlinear elements when harmonic voltages are applied to them.

Although due to the variety of network conditions and nonlinearities, no general technique is developed to determine these parameters, specific convergence criteria have been developed for the nonlinear elements studied in this work. Analysis of these particular cases provided a number of useful conclusions on the convergence properties of general cases. For example, harmonic voltage resonance was found to be the most influential negative factor in the iteration process. Fortunately, such resonance conditions are generally suppressed in normal power system operations.

Based on the results of this work, the following step-by-step remedies may be taken in the event of convergence difficulties:

1. Check the fundamental frequency load flow results to verify that the initial starting conditions of the iteration are reasonable.

2. Examine the possibility of harmonic voltage resonance. This can be done by inspecting the harmonic voltages at the terminals of the nonlinear elements obtained after the first iteration step, or by performing non-iterative frequency scan calculations.

3. If no resonance is found, alternative modelling techniques for nonlinear elements can be examined, as for example, the Norton equivalent circuit, the current source 
or even the voltage source models.

4. If none of these approaches works, the degree of nonlinearity and the PQ, PV constraints may be relaxed to see the combined effects of $c_{1}$ and $c_{2}$. Furthermore, numerical sensitivity techniques may be used to determine the approximate range of parameters $c_{1}$ and $c_{2}$. These evaluations may tell whether the divergence is caused by the iteration scheme or by the nonexistence of solutions.

The convergence analysis presented in this chapter is far from providing final conclusions, but rather a starting point for further research. As illustrated with many examples, it is always possible in one way or another to evaluate the contraction parameters. Some of the approaches can even be directly extended to more complicated cases. 


\section{Chapter 8}

\section{Conclusions}

The growth of harmonic distortion in power systems has led to an increasing concern in the power industry. Harmonic load flow analysis plays a vital role in assisting the investigation of such distortions. In view of the importance and present progress of harmonic load flow methods, a multiphase harmonic load flow program MHLF has been developed in this work. The major contributions of this research are:

1. Two numerical techniques for power system steady-state analysis have been developed." The first technique, the multiphase load flow program, is a significant advancement over the existing ones in the area of unbalanced load flow analysis at the fundamental frequency. The second technique, the multiphase harmonic load flow (MHLF) program, is developed on the basis of the first technique. The MHLF technique is the first of its kind for systematic harmonic load flow analysis of multiphase power systems.

2. Three nonlinear elements, nonlinear inductor, synchronous machine and static compensator, have been analyzed by the MHLF program. New models were developed for these elements. The nonlinear inductor and synchronous machine models were built-in directly into the program. The built-in machine models include the machine load flow constraints and the effects of saturation. The static compensators including their control characteristics are analyzed as examples of applying the MHLF program for the harmonic analysis of user selected nonlınearities. 
3. A number of case studies were performed in this work. The results not only demonstrate the effectiveness of the proposed techniques, but also indicate the importance of a multiphase approach in solving the problem of harmonic analysis under both balanced and unbalanced conditions. The results obtained in the static compensator unbalanced harmonic studies also provide useful information on the performance of the various harmonic reduction schemes.

4. The MHLF was developed on the basis of a harmonic iteration scheme. The convergence property of this scheme was analyzed on a theoretical basis. The investigation provided a unified understanding on the iterative solution of the three different nonlinear characteristics studied in this work. Factors that affect convergence are identified. Convergence criteria were derived for some simplified cases.

In this dissertation, the advantages of the MHLF technique are discussed in detail and illustrated by a number of examples. They can be summarized as follows:

- It is a general purpose multiphase program for power system steady-state analysis.

- The technique's extendibility allows easy analysis of other nonlinear elements.

- A wide range of application options is provided for harmonic analysis.

- The technique is computationally efficient.

For future research, the following topics may be suggested:

- With respect to the solution techniques, a more detailed convergence analysis is necessary in connection with developing acceleration schemes for the harmonic iteration. 
- To achieve a wider range of applications, the MHLF technique can be extended with advanced load flow features such as remote voltage regulation and on-line transformer tap changing. Also, other popular nonlinear devices, such as three-phase full wave bridge rectifiers, could be included in the program as built-in elements.

- The construction of frequency-domain models of nonlinear elements is essential for the use of the MHLF technique. This technique can be significantly improved if a general method to derive these models is developed and built into the program. 


\section{References}

[1] R.C. Dugan and D.T. Rizy, "Harmonic Considerations for Electrical Distribution Feeders", Technical Report ORNL/Sub/81-95011/4, Oak Ridge National Laboratory, Oak Ridge, Tennessee 37831, Mar. 1988.

[2] IEEE Working Group on Power System Harmonics, "Power System Harmonics: An Overview", IEEE Transactions on Power Apparatus and Systems, vol. PAS102, pp. 2455-2460, Aug. 1983.

[3] T.J. Densem, "Three Phase Power System Harmonic Penetration", Ph.D. Dissertation, University of Canterbury, Christchurch, New Zealand, 1983.

[4] T.J. Aprille and T.N. Trick, "Steady-State Analysis of Nonlinear Circuits with Periodic Inputs", Proceedings of the IEEE, vol. 60, pp. 108-114, Jan. 1972.

[5] M.S. Nakhala and F.H. Branin, "Determining the Periodic Responses of Nonlinear Systems by a Gradient Method", International Journal on Circuit Theory Application, vol. 5, pp. 255-273, 1977.

[6] S. Skelboe, "Computation of the Periodic Steady-State Response of Nonlinear Networks by Extrapolation Methods", IEEE Transactions on Circuits and Systems, vol. CAS-27, pp. 161-175, Mar. 1980.

[7] E.M. Baily, "Steady State Harmonic Analysis of Nonlinear Network", Ph.D. Dissertation, Stanford University, Stanford, Calif., 1968.

[8] A. Ushida and L.O. Chua "Frequency-Domain Analysis of Nonlinear Circuits Driven by Multi-tone Signals", IEEE Transactions on Circuits and Systems, vol. CAS-31, pp. 766-778, Sept. 1984. 
[9] H.W. Dommel, "Digital Computer Solution of Electromagnetic Transients in Single and Multiphase Networks", IEEE Transactions on Power Apparatus and Systems, vol. PAS-88, No. 4, pp. 388-399, Apr. 1969.

[10] H.W. Dommel, et al Case Studies for Electromagnetic Transients, Dept. of Electrical Engineering, University of British Columbia, Dec., 1986.

[11] R.H. Kitchin "Convertor Harmonics in Power Systems Using State-variable Analysis", IEE Proceedings, vol. 128, Part C, No. 4, July 1981.

[12] H.W. Dommel, Electromagnetic Transients Program Reference Manual (EMTP Theory Book), Prepared for Bonneville Power Administration, Dept. of Electrical Engineering, University of British Columbia, Aug. 1986.

[13] R. Yacamini and J.C. de Oliveira "Harmonics in Multiple Convertor Systems: a Generalized Approach", IEE Proceedings, vol. 127, Part B, No. 2, Mar. 1980.

[14] H.W. Dommel, A. Yan and W. Shi, "Harmonics from Transformer Saturation", IEEE Transactions on Power Delivery, vol. PWRD-1, pp. 209-215, Apr. 1986.

[15] D. Xia and G.T. Heydt, "Harmonic Power Flow Studies, Part I - Formulation and Solution, Part II - Implementation and Practical Application", IEEE Transactions on Power Apparatus and Systems, vol. PAS-101, pp. 1257-1270, June 1982.

[16] W. Song, G.T. Heydt and W.M. Grady, "The Integration of HVDC Subsystems into Harmonic Power Flow Algorithm", IEEE Transactions on Power Apparatus and Systems, vol. PAS-103, pp. 1953-1961, Aug. 1984.

[17] A.H. El-Abiad and D.C. Tarsi, "Load Flow Solution of Untransposed EHV Networks", PICA, Pittsburgh, pp. 377-384, 1967.

[18] J. Arrillaga, C.P. Arnold and B.J. Harker, Computer Modeling of Electrical Power Systems, New Zealand: John Wiley\&Sons, 1983. 
[19] N.A. Wortman, D.L. Allen and L.L. Grigsby, "Techniques for the Steady State Representation of Unbalanced Power Systems", IEEE Transactions on Power Apparatus and Systems, vol. PAS-104, pp. 2805-2824, Oct. 1985.

[20] T.J. Miller, Ed. Reactive Power Control in Electric Systems, New York: John Wiley\&Sons Inc., 1982.

[21] G.W. Stagg and A.H. El-Abiad, Computer Methods in Power System Analysis, New York: McGraw-Hill Book Company, 1968.

[22] CIGRE-Working Group 36-05, "Harmonics, Characteristic Parameters, Methods of Study, Estimates of Existing Values in the Network", Electra, no. 77, pp. 35-54, July 1981 .

[23] W. Xu and H.W. Dommel "Computation of Steady-State Harmonics of Static Var Compensators", Proc. of the Third International Conference on Harmonics in Power Systems, Nashville, IN, Oct. 1988, pp. 239-245.

[24] D.M. Young and R.T. Gregory, A Survey of Numerical Mathematics, vol. I, Reading, Massachusetts: Addison-Wesley Publishing Company, 1972.

[25] Substation Standards and Performance, TransAlta Utilities Corporation, "Part I - Data Related to the $240 \mathrm{kV}$ Southern Alberta Transmission Network and Langdon Substation, Part II - Results of Field Measurements of Steady-State Voltage and Current Harmonics for Various SVC Operating Conditions", Internal Report, TransAlta Utilities Corp., Feb. 1986.

[26] T.K.P. Medicherla, "Second Order Load Flow Technique", M.Sc. Thesis, Dept. of Electrical Engineering, University of Saskatchewan, Sask., 1975.

[27] I.D. Lu, "Tumbler Ridge Unbalance and Harmonics 1986", Internal Report of RED Project No. 8711-86, B.C. Hydro, May 1987.

[28] W.E. Dillon and M.S. Chen, "Power System Modelling", Proceedings IEEE, pp. 539-554, July 1974. 
[29] A.S. Morched and P. Kundur, "Identification and Modeling of Load Characteristics at High Frequencies", IEEE Transactions on Power Systems, vol. PS-2, pp. 153160, Feb. 1987.

[30] G.L. Berg, "Power System Load Representation", Proc. IEE, vol. 120, pp. 344-348, Mar. 1973.

[31] W.F. Tinney and C.E. Hart, "Power Flow Solution by Newton's Method", IEEE Transactions on Power Apparatus and Systems, vol. PAS-86, pp. 1449-1460, Nov. 1967.

[32] M.B. Hughes, "Tumbler Ridge Harmonics Study", Diary of M.B. Hughes, B.C. Hydro, 16 March 1988.

[33] P.M. Anderson, Analysis of Faulted Power Systems, Ames, Iowa: The Iowa State University Press, 1973.

[34] A. Semlyen, E. Acha, and J. Arrillaga, "Harmonic Norton Equivalent for the Magnetising Branch of a Transformer", IEE Proceedings, Vol.134, Part C, No.2, pp. 162-169, March 1987.

[35] W.M. Grady, "Harmonics in Power Systems", IEEE Tutorial Course: Power System Harmonics, 84 EH0221-2-PWR, pp. 11-20, 1984.

[36] J.D. Roark and C.A. Gross, "Unbalanced Synchronous Machine Analysis Using Frequency Domain Methods", IEEE Paper No. 78 SM 524-1, Presented at the 1978 IEEE Summer Power Meeting, Los Angeles, CA.

[37] A. Semlyen, J.F. Eggleston and J. Arrillaga, "Admittance Matrix Model of a Synchronous Machine for Harmonic Analysis", IEEE Transactions on Power Systems vol. PS-2, pp. 833-840, Nov. 1987.

[38] R.T. Smith, Analysis of Electrical Machines, New York: Pergamon Press, 1982. 
- [39] A.P. Meliopoulos, K.R. Chakravarthi, and T. Ortmeyer, "Effects of Harmonics on Power System Equipment and Loads", IEEE Tutorial Course: Power System Harmonics, 84 EH0221-2-PWR, pp. 21-31, 1984.

[40] G.H. Golub and C.F. Van Loan, Matrix Computations, the Johns Hopkins University Press, Baltimore, Maryland, 1983.

[41] W. W. Sawyer, A First Look at Numerical Functional Analysis, Clarendon Press, Oxford, 1978.

[42] J. A. Jensen and J.H. Rowland, Methods of Computation - the Linear Space Approach to Numerical Analysis, Scott, Foresman and Company, Glenview, Hlinois, 1978.

[43] R. L. Burden, J.D. Faires and A. C. Reynolds, Numerical Analysis, Prindle, Weber\&Schmidt, Inc., Boston, Massachusetts, 1978. 


\section{Appendix A}

\section{DQO-machine Model with Saturation}

\section{A.1 Flux Linkage Equation with Saturation}

To facilitate the explanation, assume that all machine quantities are described in perunit. It is also assumed that the saturation curve has been scaled so that the unsaturated curve is at a $45^{\circ}$ angle as shown in Figure A.1.

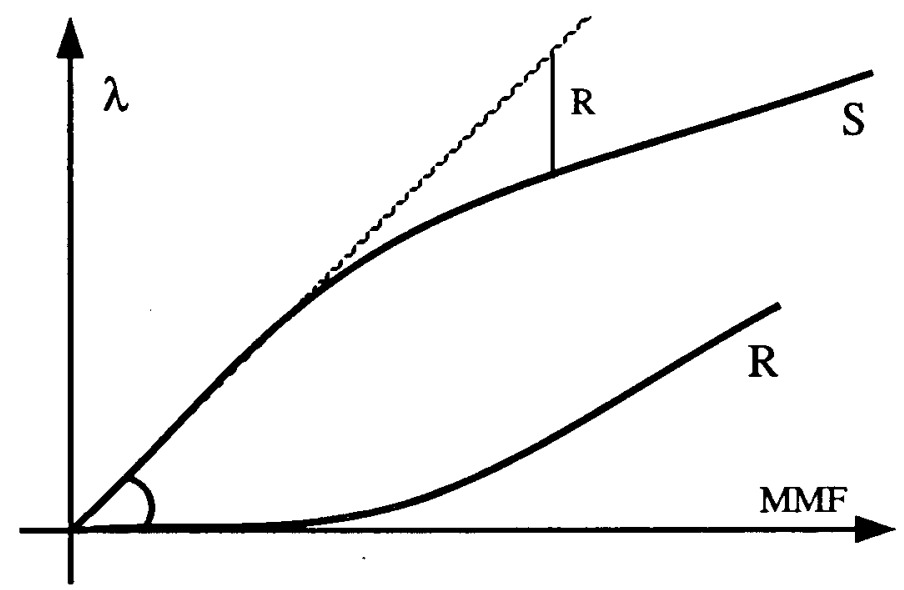

Figure A.1: Normalized saturation curve of a synchronous machine.

This scaling leads the magnetomotive force (MMF) equal to the unsaturated flux. As 
a result, the MMF's at instant $t$ can be obtained as:

$$
\begin{aligned}
& f_{d}(t)=M_{d}\left(i_{d}+i_{f}+i_{D}\right) \\
& f_{q}(t)=M_{q}\left(i_{q}+i_{g}+i_{Q}\right) .
\end{aligned}
$$

where $f_{d}(t)$ and $f_{q}(t)$ represent the MMF's along $d$ - and q-axes, respectively. $M_{d}$ and $M_{q}$ are the unsaturated mutual inductances in $\mathrm{d}$ - and $\mathrm{q}$-axes and are related to the unsaturated inductance matrix $[L]$ in the following form:

$$
[L]=\left[\begin{array}{lllllll}
L_{l}+M_{d} & 0 & 0 & M_{d} & 0 & M_{d} & 0 \\
0 & L_{l}+M_{q} & 0 & 0 & M_{q} & 0 & M_{q} \\
0 & 0 & L_{0} & 0 & 0 & 0 & 0 \\
M_{d} & 0 & 0 & L_{f l}+M_{d} & 0 & M_{d} & 0 \\
0 & M_{q} & 0 & 0 & L_{g l}+M_{q} & 0 & M_{q} \\
M_{d} & 0 & 0 & M_{d} & 0 & L_{D l}+M_{d} & 0 \\
0 & M_{q} & 0 & 0 & M_{q} & 0 & L_{Q l}+M_{q}
\end{array}\right],
$$

where subscript $l$ indicates leakages. Note that this matrix is different with the $[L]$ matrix described in Chapter 5 because of the per-unit scaling. The total air-gap MMF, $f_{\text {total }}(t)$, is

$$
f_{\text {total }}(t)=\sqrt{f_{d}(t)^{2}+f_{q}(t)^{2}} .
$$

It is this MMF that generates air-gap flux through the function of saturation [12] shown in Figure A.1, as follows:

$$
\begin{aligned}
\lambda_{\text {total }}(t) & =S\left(f_{\text {total }}(t)\right) \\
& =f_{\text {total }}(t)-R\left(f_{\text {total }}(t)\right) .
\end{aligned}
$$


The air-gap fluxes along the $d$ - and $q$-axes are then

$$
\begin{aligned}
\lambda_{m d}(t) & =\left[f_{d}(t) / f_{\text {total }}(t)\right] \lambda_{\text {total }}(t) \\
& =f_{d}(t)-\left[f_{d}(t) / f_{\text {total }}(t)\right] R\left(f_{\text {total }}(t)\right) \\
& =f_{d}(t)-w_{d}(t) \\
\lambda_{m q}(t) & =\left[f_{q}(t) / f_{\text {total }}(t)\right] \lambda_{\text {total }}(t) \\
& =f_{q}(t)-\left[f_{q}(t) / f_{\text {total }}(t)\right] R\left(f_{\text {total }}(t)\right) \\
& =f_{q}(t)-w_{q}(t),
\end{aligned}
$$

where

$$
\begin{aligned}
& w_{d}(t)=\left[f_{d}(t) / f_{\text {total }}(t)\right] R\left(f_{\text {total }}(t)\right) \\
& w_{q}(t)=\left[f_{q}(t) / f_{\text {total }}(t)\right] R\left(f_{\text {total }}(t)\right) .
\end{aligned}
$$

It can be seen that, due to the saturation, both the air-gap fluxes in $d$ - and q-axes are reduced by the amount of $w_{d}$ and $w_{q}$, determined by the nonlinear function $R$. With this modification, the flux linkage equation becomes

$$
\left[\lambda_{p a r k}\right]=\left[\begin{array}{l}
L_{l} i_{d}+\lambda_{m d} \\
L_{l} i_{q}+\lambda_{m q} \\
L_{o} i_{o} \\
L_{f l} i_{f}+\lambda_{m d} \\
L_{g l} i_{g}+\lambda_{m q} \\
L_{D l} i_{D}+\lambda_{m d} \\
L_{Q l} i_{Q}+\lambda_{m q}
\end{array}\right]=\left[\begin{array}{l}
L_{l} i_{d}+f_{d} \\
L_{l} i_{q}+f_{q} \\
L_{o} i_{o} \\
L_{f l} i_{f}+f_{d} \\
L_{g l} i_{g}+f_{q} \\
L_{D l} i_{D}+f_{d} \\
L_{Q l} i_{Q}+f_{q}
\end{array}\right]-\left[\begin{array}{l}
w_{d} \\
w_{q} \\
0 \\
w_{d} \\
w_{q} \\
w_{d} \\
w_{q}
\end{array}\right] .
$$


According to Eq. (A.3), Eq. (A.7) can be simplified as:

$$
\left[\lambda_{\text {park }}\right]=[L]\left[i_{p a r k}\right]-[E]\left[w_{p a r k}\right]
$$

where

$$
\begin{gathered}
{[E]=\left[\begin{array}{ccccccc}
1 & 0 & 0 & 1 & 0 & 1 & 0 \\
0 & 1 & 0 & 0 & 1 & 0 & 1
\end{array}\right]^{T}} \\
{\left[w_{\text {park }}\right]=\left[\begin{array}{ll}
w_{d} & w_{q}
\end{array}\right]^{T}}
\end{gathered}
$$

Eq. (A.8) is the flux linkage equation with saturation included, which is similar to Eq. (5.2) of Chapter 5 but with additional correction term $\left[w_{\text {park }}\right]$.

\section{A.2 Machine Model in DQO-coordinates}

The machine dynamic equation, Eq. (5.1) (Section 5.3), remains unchanged even with saturation. To relate the voltages to the currents in dqo-coordinates, the new flux linkage equation (Eq. (A.8)) is substituted into Eq. (5.1), which yields

$$
\begin{aligned}
{\left[v_{\text {park }}\right] } & =-[R]\left[i_{\text {park }}\right]-p\left[\lambda_{\text {park }}\right]+[F]\left[\lambda_{\text {park }}\right] \\
& =(-[R]-p[L]+[F][L])\left[i_{\text {park }}\right]+(p[U]-[F])[E]\left[w_{\text {park }}\right] \\
& =[Z(p)]\left[i_{\text {park }}\right]+[G(p)]\left[w_{\text {park }}\right]
\end{aligned}
$$

where

$$
[G(p)]=(p[U]-[F])[E]
$$

and $[U]$ is a $7 \times 7$ unit matrix. 
As shown above, Eq. (A.9) is similar to Eq. (5.3) of Chapter 5 except an additional term $[G(p)]\left[w_{p a r k}\right]$. Since $\left[w_{p a r k}\right]$ is an nonlinear function of $\left[i_{p a r k}\right]$, Eq. (A.9) describes an nonlinear dynamic system. Consequently, previously used technique of frequency analysis can not be directly applied.

It is noted, however, that $\left[w_{\text {park }}\right]$ is a periodic time function in steady-states. It can be assumed therefore that $\left[w_{\text {park }}\right]$ is represented by a known discrete Fourier expansion

$$
\left[w_{p a r k}(t)\right]=\operatorname{Real}\left\{\sum_{h=0}^{n+1} \sqrt{2}[\mathbf{W}(h)] e^{j h \omega t}\right\} .
$$

Replacing the $\left[w_{\text {park }}\right]$ term in Eq. (A.9) with above expression, Eq. (A.9) becomes a linear system with harmonic excitation. The frequency domain analysis can again be performed. For the case of $h \neq 0$, it leads to

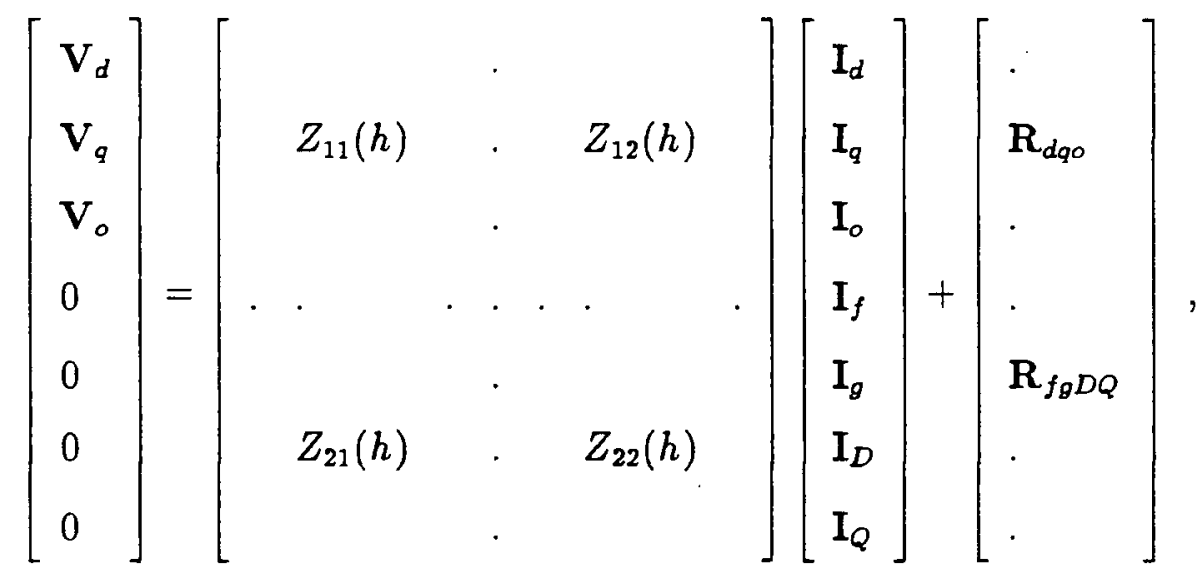

where

$$
\left[\begin{array}{l}
\mathbf{R}_{d g \circ} \\
\mathbf{R}_{f g D Q}
\end{array}\right]=[G(h)][\mathbf{W}(h)] .
$$

Solving for $\left[\mathbf{I}_{d q o}(h)\right]$ as a function of $\left[\mathbf{V}_{d q o}(h)\right]$ and $[\mathbf{W}(h)]$ yields

$$
\left[\mathbf{I}_{d q o}(h)\right]=\left[Y_{d q o}(h)\right]\left[\mathbf{V}_{d q o}(h)\right]+\left[\mathbf{I}_{d q o-s}(h)\right]
$$


where

$$
\left[\mathbf{I}_{q d o-s}(h)\right]=\left[Y_{d q o}(h)\right]\left(\left[\mathbf{R}_{d q o}\right]-\left[Z_{12}(h)\right]\left[Z_{22}(h)\right]^{-1}\left[\mathbf{R}_{f g D Q}\right]\right)
$$

Equation (A.10) is the required machine model with saturation. Therefore, the effects of saturation can be simply represented as a known current source in parallel with the dqo-machine model developed in Section 5.3.

\section{A.3 A Subiteration Process}

It has been assumed in the last subsection that the discrete Fourier expansion of $\left[w_{\text {park }}\right]$ is known. With this assumption, the nonlinear dynamic system is converted into a linear one. This subsection describes a numerical process to determine that Fourier expansion.

According to the development in last subsection, a machine model in dqo-coordinates can be represented by Eq. (A.9), namely

$$
\left[v_{\text {park }}\right]=[Z(p)]\left[i_{\text {park }}\right]+[G(p)]\left[w_{\text {park }}\right]
$$

It has also been shown that $\left[w_{p a r k}\right]$ are the nonlinear algebraic function of vector $\left[i_{p a r k}\right]$. There are only two variables, $\left[v_{p a r k}\right]$ and $\left[i_{p a r k}\right]$, in the equation therefore. The idea is to solve for the vector $\left[i_{p a r k}\right]$ in the frequency-domain from Eq. (A.12) given the voltage $\left[v_{p a r k}\right]$ that is the result of previous load flow run. The solution is obtained with a subiteration process, as listed in following steps.

1. Compute all harmonic components of $\left[v_{p a r k}\right]$ from the load flow results, and assume $\left[w_{\text {park }}\right]=0$. 
2. Solve for all harmonic components of $\left[i_{\text {park }}\right]$ by using Eq. (A.12) in frequency domain.

3. Compute $f_{d}(t), f_{q}(t)$ and $f_{\text {total }}(t)$ according to Eqs. (A.1), (A.2), and (A.4), and then compute $w_{d}(t)$ and $w_{q}(t)$ according to Eqs. (A.5) and (A.6), all point-bypoint in time domain. Waveforms of a half cycle of the fundamental frequency are sufficient.

4. The point-by-point results of $w_{d}(t)$ and $w_{q}(t)$ are then analyzed using DFT to provide $[\mathbf{W}(h)]$ in frequency domain.

5. If the obtained $[\mathbf{W}(h)]$ is close to the one in last subiteration, then stop iteration; otherwise replace $[\mathbf{W}(h)]$ with the newly computed results and go to step 2 .

This process is similar to the one used to solve the harmonic problem of nonlinear inductors. After its convergence, the subiteration provides the $[\mathbf{W}(h)]$ solution that is accurate for the given voltage conditions. 


\section{Appendix B}

\section{Proof of Convergence Theorems}

\section{Proof of Theorem 1}

\section{(1) Existence.}

If $f(a)=c$ and $g(c)=a$ or $f(b)=d$ and $g(d)=b$, the existence of a solution point is obvious. Suppose this is not the case, then it must be true that $g(f(a))>a$ and $g(f(b))<b$. Define

$$
h(x)=g(f(x))-x
$$

$h$ is continuous on $\Omega_{x}$, and moreover, $h(a)>0, h(b)<0$. The Intermediate Value Theorem [43] implies that there exists a point $\alpha \in \Omega_{x}$, for which $h(\alpha)=0$, that is $f(\alpha)=\beta$, $g(\beta)=\alpha$.

\section{(2) Uniqueness.}

Assume that there are two different solutions $\left(\alpha_{1}, \beta_{1}\right)$ and $\left(\alpha_{2}, \beta_{2}\right)$. According to the Mean Value Theorem [43], there are $\xi \in \Omega_{x}$ and $\zeta \in \Omega_{y}$ such that

$$
\begin{aligned}
\left|\alpha_{1}-\alpha_{2}\right| & =\left|g\left(\beta_{1}\right)-g\left(\beta_{2}\right)\right| \leq\left|g^{\prime}(\zeta)\right|\left|\beta_{1}-\beta_{2}\right| \\
& =\left|g^{\prime}(\zeta)\right|\left|f\left(\alpha_{1}\right)-f\left(\alpha_{2}\right)\right| \leq\left|g^{\prime}(\zeta)\right|\left|f^{\prime}(\xi)\right|\left|\alpha_{1}-\alpha_{2}\right|
\end{aligned}
$$


Applying condition 2 to $g^{\prime}(\zeta)$ and $f^{\prime}(\xi)$, yields

$$
\left|\alpha_{1}-\alpha_{2}\right| \leq c_{1} c_{2}\left|\alpha_{1}-\alpha_{2}\right|<\left|\alpha_{1}-\alpha_{2}\right|
$$

which is a contradiction. Hence the solution point is unique.

\section{(3) Convergence.}

According to Eq. (7.2) and the Mean Value Theorem,

$$
\begin{aligned}
\left|x_{i+1}-\alpha\right| & =\left|g\left(y_{i}\right)-g(\beta)\right| \leq\left|g^{\prime}(\zeta)\right|\left|y_{i}-\beta\right| \\
& =\left|g^{\prime}(\zeta)\right|\left|f\left(x_{i}\right)-f(\alpha)\right| \leq\left|g^{\prime}(\zeta)\right|\left|f^{\prime}(\xi)\right|\left|x_{i}-\alpha\right| \\
& \leq c_{1} c_{2}\left|x_{i}-\alpha\right|=c\left|x_{i}-\alpha\right|
\end{aligned}
$$

Applying above relationship inductively gives

$$
\left|x_{i}-\alpha\right| \leq c\left|x_{i-1}-\alpha\right| \leq \ldots \leq c^{i}\left|x_{o}-\alpha\right|
$$

Since $c<1$

$$
\lim _{n \rightarrow \infty}\left|x_{i}-\alpha\right| \leq \lim _{n \rightarrow \infty} c^{i}\left|x_{o}-\alpha\right| \leq 0
$$

That is, iteration (7.2) converges to $\alpha$.

(4) Rate of convergence.

Again applying the Mean Value Theorem and the given conditions yields

$$
\left|x_{i+1}-x_{i}\right| \leq c\left|x_{i}-x_{i-1}\right| \leq \ldots \leq c^{i}\left|x_{1}-x_{0}\right|
$$


Thus for $m>i>1$

$$
\begin{aligned}
\left|x_{m}-x_{i}\right| & =\left|x_{m}-x_{m-1}+x_{m-1}-\ldots+x_{i+1}-x_{i}\right| \\
& \leq\left|x_{m}-x_{m-1}\right|+\left|x_{m-1}-x_{m-2}\right|+\ldots+\left|x_{i+1}-x_{i}\right| \\
& \leq\left(c^{m-1}+c^{m-2}+\ldots+c^{i}\right)\left|x_{1}-x_{0}\right| \\
& =c^{i}\left(c^{m-i-1}+c^{m-i-2}+\ldots+c+1\right)\left|x_{1}-x_{0}\right|
\end{aligned}
$$

Since $\lim _{m \rightarrow \infty} x_{m}=\alpha$,

$$
\left|\alpha-x_{i}\right|=\lim _{m \rightarrow \infty}\left|x_{m}-x_{i}\right| \leq c^{i}\left|x_{1}-x_{0}\right| \sum_{k=0}^{\infty} c^{k}=\frac{c^{i}}{1-c}\left|x_{1}-x_{0}\right| .
$$

\section{Proof of Theorem 2}

The proof is quite similar to the proof of Theorem 1. According to the given bounding conditions

$$
\begin{aligned}
\left\|\left[i_{i+1}\right]-[\alpha]\right\| & =\left\|g\left(\left[v_{i}\right]\right)-g([\beta])\right\| \leq c_{2}\left\|\left[v_{i}\right]-[\beta]\right\| \\
& =c_{2}\left\|[R]\left(\left[i_{i}\right]-[\alpha]\right)\right\| \leq c_{2}\|[R]\|\left\|\left[i_{i}\right]-[\alpha]\right\| \\
& =c_{1} c_{2}\left\|\left[i_{i}\right]-[\alpha]\right\|
\end{aligned}
$$

By inductively using above relationship and considering $c=c_{1} c_{2}<1$,

$$
\lim _{n \rightarrow \infty}\left\|\left[i_{i}\right]-[\alpha]\right\|=\lim _{n \rightarrow \infty} c^{i}\left\|\left[i_{o}\right]-[\alpha]\right\|=0
$$

So that the iterative process converges to the solution point.

\section{Proof of Theorem 3}


This proof is based on the fixed point theory in generalized vector space [41, 42]. With the given conditions, the distance between the results from two successive iterations are

$$
\begin{aligned}
\left\|\left[i_{2}\right]-\left[i_{1}\right]\right\| & =\left\|T_{2}\left(\left[v_{1}\right]\right)-T_{2}\left(\left[v_{o}\right]\right)\right\| \leq c_{2}\left\|\left[v_{1}\right]-\left[v_{\mathrm{o}}\right]\right\| \\
& =c_{2}\left\|T_{1}\left(\left[i_{1}\right]\right)-T_{1}\left(\left[i_{\mathrm{o}}\right]\right)\right\| \leq c_{1} c_{2}\left\|\left[i_{1}\right]-\left[i_{\mathrm{o}}\right]\right\| \\
& =c\left\|\left[i_{1}\right]-\left[i_{\mathrm{o}}\right]\right\| .
\end{aligned}
$$

Similarly

$$
\left\|\left[i_{i+1}\right]-\left[i_{i}\right]\right\| \leq c \mid\left\|\left[i_{i}\right]-\left[i_{i-1}\right]\right\| \leq \ldots \leq c^{i}\left\|\left[i_{1}\right]-\left[i_{0}\right]\right\| .
$$

Now if $m>i>1$, we have

$$
\begin{aligned}
\left\|\left[i_{m}\right]-\left[i_{i}\right]\right\| & =\left\|\left[i_{m}\right]-\left[i_{m-1}\right]+\left[i_{m-1}\right]-\ldots+\left[i_{i+1}\right]-\left[i_{i}\right]\right\| \\
& \leq\left\|\left[i_{m}\right]-\left[i_{m-1}\right]\right\|+\left\|\left[i_{m-1}\right]-\left[i_{m-2}\right]\right\|+\ldots+\left\|\left[i_{i+1}\right]-\left[i_{i}\right]\right\| \\
& \leq\left(c^{m-1}+c^{m-2}+\ldots+c^{i}\right)\left\|\left[i_{1}\right]-\left[i_{o}\right]\right\| \\
& \leq \sum_{k=i}^{\infty} c^{k}\left\|\left[i_{1}\right]-\left[i_{0}\right]\right\|=\frac{c^{i}}{1-c}\left\|\left[i_{1}\right]-\left[i_{o}\right]\right\| .
\end{aligned}
$$

Since $c<1$, for any given $\varepsilon$, we can always find a number $N, m>i>N$, such that $\left\|\left[i_{m}\right]-\left[i_{i}\right]\right\|<\varepsilon$. Thus $\left\{\left[i_{i}\right]\right\}$ is a Cauchy sequence and converges to a limit. Due to conditions 1 and 2 , this limit is the solution point $[\alpha]$.

In order to show that $[\alpha]$ is the only solution, suppose $\left[\alpha^{\prime}\right]$ is a different solution, then

$$
\begin{aligned}
\left\|[\alpha]-\left[\alpha^{\prime}\right]\right\| & =\left\|T_{2}([\beta])-T_{2}\left(\left[\beta^{\prime}\right]\right)\right\| \leq c_{2}\left\|[\beta]-\left[\beta^{\prime}\right]\right\| \\
& =c_{2}\left\|T_{1}([\alpha])-T_{1}\left(\left[\alpha^{\prime}\right]\right)\right\| \leq c_{1} c_{2}\left\|[\alpha]-\left[\alpha^{\prime}\right]\right\|<\left\|[\alpha]-\left[\alpha^{\prime}\right]\right\| .
\end{aligned}
$$

This contradiction rules out the possibility of another solution point. 


\section{Appendix C}

\section{Multiple Solutions in Unbalanced Load Flow Analysis}

It was observed during the course of this work that multiple solutions exist in some unbalanced load flow studies. Although the problem of multiple solution is not new for the single-phase load flow analysis, it has some distinct characteristics in the unbalanced load flow cases. One of such characteristics is that the solutions are dependent on the degree of network unbalance and the load models. The purpose of this Appendix is to present some typical observations on the problem. These observations may be useful to stimulate the future research on the subject, and also to provide guidelines for the related load flow studies.

The observed multiple solution problems are all related to the individual-phase load (type-2) model. If the load models are arranged in the form shown in Figure C.1, two solutions can be obtained for the voltage at the star-point $\mathrm{N}$.

To compute the voltage at node $\mathrm{N}, \mathrm{V}_{N}$, following equation can be established:

$$
\mathbf{I}_{a}+\mathbf{I}_{b}+\mathbf{I}_{c}=0 .
$$

Substituting the loads and source voltages yields 


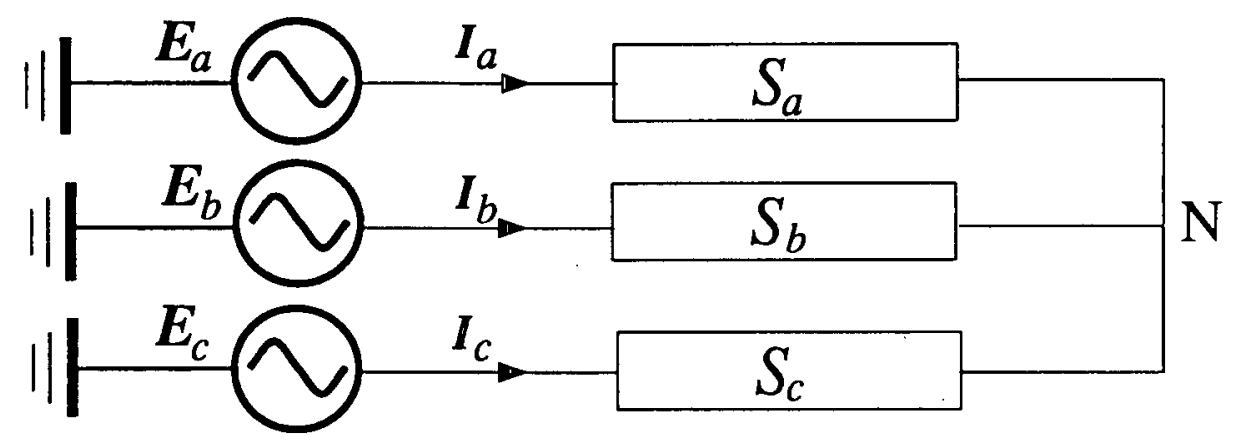

Figure C.1: Load arrangement that causes multiple solutions.

$$
\frac{S_{a}}{\mathbf{E}_{a}-\mathbf{V}_{N}}+\frac{S_{b}}{\mathbf{E}_{b}-\mathbf{V}_{N}}+\frac{S_{c}}{\mathbf{E}_{c}-\mathbf{V}_{N}}=0
$$

This is the load flow equation that has multiple solutions. The solutions are illustrated by following two unbalanced cases.

Case 1: Voltage unbalance related multiple solution.

It is assumed in this case that only the source voltages are unbalanced:

$$
\begin{gathered}
\mathbf{E}_{a}=1-\alpha, \quad \mathbf{E}_{b}=e^{-j 120^{\circ}}, \quad \mathbf{E}_{c}=e^{-j 240^{\circ}}, \\
S_{a}=S_{b}=S_{c}
\end{gathered}
$$

With these conditions, Eq. (C.2) can be further simplified. It results in following equation:

$$
3 \mathbf{V}_{N}^{2}+2 \alpha \mathbf{V}_{N}+\alpha=0
$$

This equation is a quadratic equation. There are two solutions for it in theory. In order to view the property of the solutions, function $f$ is defined:

$$
f=3 \mathbf{V}_{N}^{2}+2 \alpha \mathbf{V}_{N}+\alpha
$$



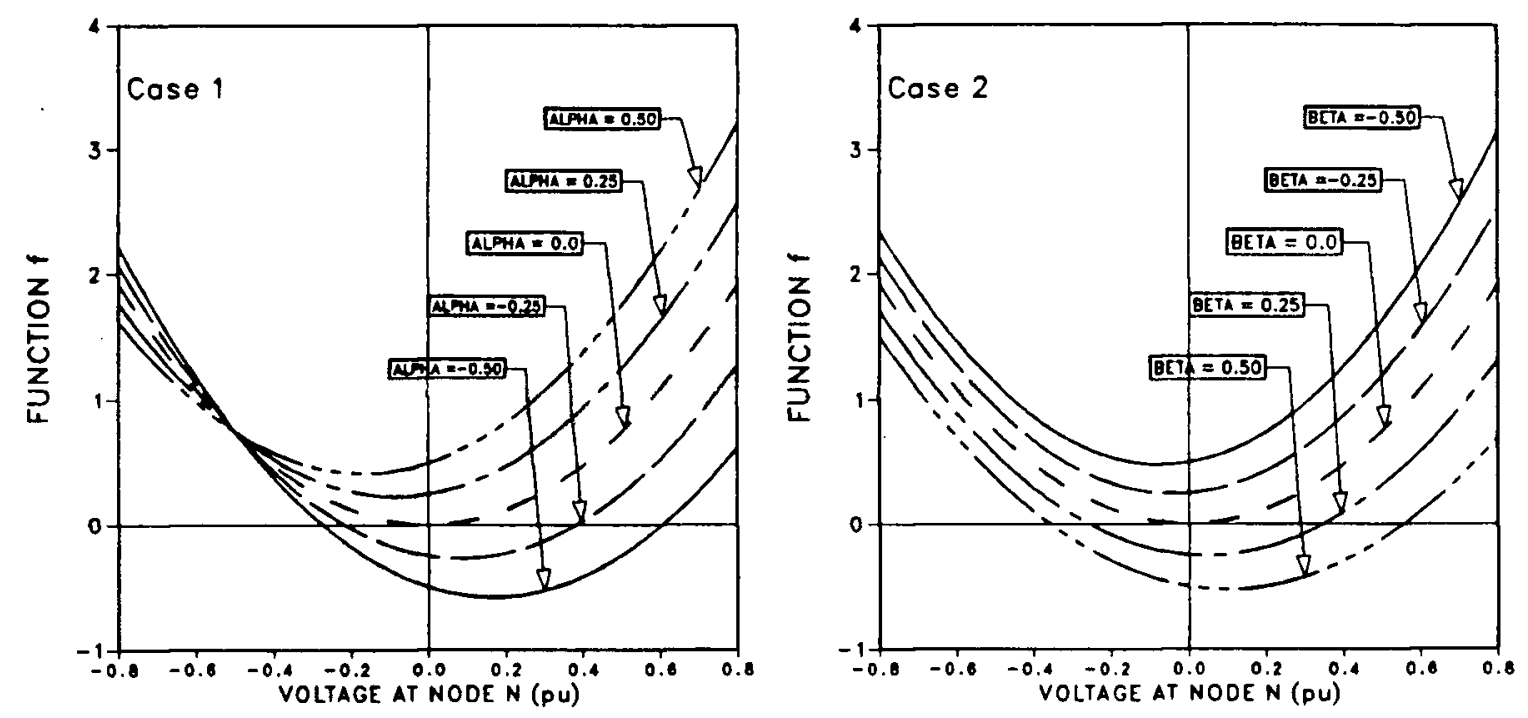

Figure C.2: Solution curves of two illustrated cases.

The plot of $f$ as a function of $\mathrm{V}_{N}$ is shown in Figure C.2(case 1). Each curve in the figure represents the $f \sim V_{N}$ function with different unbalance parameter $\alpha$. The solutions are the intersection points of the curves with the $\mathrm{x}$-axis, that is $f=0$. As shown in the figure, there exist two real solutions for some values of parameter $\alpha$. Inspection of the curves and Eq. (C.3) may lead to following preliminary conclusions:

1. The solution points are independent of the load conditions. It is purely related to the degree of unbalance of the source voltages.

2. If the sources are balanced, two solution points merge into one point, $\mathbf{V}_{N}=0$. The two solutions are quite close if the degree of unbalance is small.

3. A pair of conjugate complex solutions exist for $\alpha>0$.

Case 2: Load unbalance related multiple solution. 
In this case, it is assumed that the only unbalance is the load unbalance. One of such operating conditions is:

$$
\begin{gathered}
\mathrm{E}_{a}=1.0, \quad \mathbf{E}_{b}=e^{-j 120^{\circ}}, \quad \mathbf{E}_{c}=e^{-j 240^{\circ}} \\
S_{a}=(1-\beta) S, \quad S_{b}=S, \quad S_{c}=S
\end{gathered}
$$

With these conditions, the general equation, Eq. (C.2), can be simplified as:

$$
(3-\beta) \mathbf{V}_{N}^{2}-\beta \mathbf{V}_{N}-\beta=0
$$

Again, there are two solutions expected for this quadratic equation. The property of the solutions can be viewed from Figure C.2(case 2), which shows the $f \sim V_{N}$ curves with different unbalance parameter $\beta$. Function $f$ is defined as

$$
f=(3-\beta) \mathrm{V}_{N}^{2}-\beta \mathrm{V}_{N}-\beta
$$

As shown in the figure, most conclusions drawn for the case 1 apply to this case as well. The two solutions are directly related to the degree of unbalance, and are independent of the source voltages. To interpret the physical meaning of the two solution points, case with $\beta=1$, i.e. zero phase a load is considered. This zero load condition can be modelled as either open circuit or short circuit of phase a, as shown in Figure C.3. Accordingly there are two solutions for the problem.

Besides the above reported cases, more investigations have been done during the course of this work. The general impression obtained from these studies can be summarized as follows: 


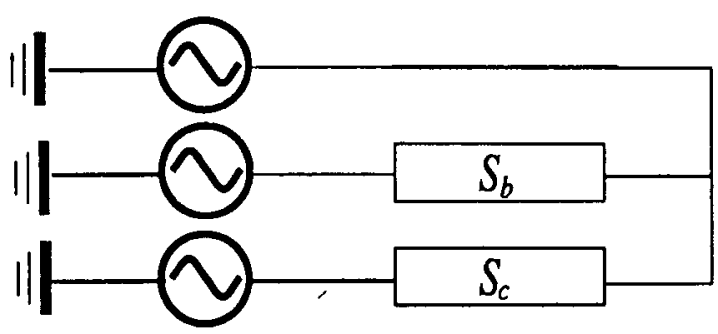

(a) short circuit

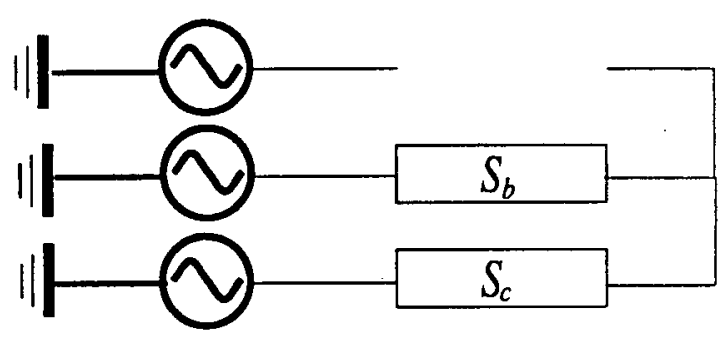

(b) open circuit

Figure C.3: Interpretation of two solutions when $\beta=1$.

1. The discussed multiple solution problems are characterized with the dependence on the degree of unbalance. If the network is balanced, the solutions merge into one point.

2. It seems that only the individual-phase load model connected in $\mathrm{Y}$ or $\mathrm{Y}$-impedanceto-ground causes such kind of multiple solution problems.

3. It is likely that the load arrangement of Figure C.1 may not be practical. Therefore, care must be taken to construct unusual load structures using the various options provided by the MHLF technique.

4. If the cases are practical, correct solution point may only be determined by considering the dynamics of the load. 


\section{Appendix D}

\section{Equivalent Circuit Models of Nonlinear Elements}

Three nonlinear elements, nonlinear inductor, synchronous machine and static compensator with TCR, have been analyzed by the MHLF program in this thesis. This appendix summarizes the harmonic Norton equivalent circuit models of these elements and the associated convergence criteria.

Table D.1: List of nonlinear elements and their equivalent circuits

\begin{tabular}{|c|c|c|c|}
\hline Elements & nonlinear inductor & synchronous machine & static compensator \\
\hline $\begin{array}{l}\text { Source of } \\
\text { Harmonics }\end{array}$ & saturation & $\begin{array}{l}\text { frequency conversion } \\
\text { and saturation }\end{array}$ & $\begin{array}{l}\text { convertor } \\
\text { operation }\end{array}$ \\
\hline $\begin{array}{l}\text { Control } \\
\text { Characteristics }\end{array}$ & none & $\begin{array}{l}\text { voltage or/and } \\
\text { power constraints }\end{array}$ & $\begin{array}{l}\text { compensator } \\
\text { characteristics }\end{array}$ \\
\hline $\begin{array}{l}\text { Equivalent } \\
\text { Circuit Model }\end{array}$ & $\begin{array}{l}\text { Figure } 4.2 \\
\text { Eq. (4.3) }\end{array}$ & $\begin{array}{l}\text { Figure } 5.1 \\
\text { Eqs. }(5.8),(5.9),(5.12)\end{array}$ & $\begin{array}{l}\text { Figure } 6.1 \\
\text { Eqs. }(6.3),(6.4)\end{array}$ \\
\hline $\begin{array}{l}\text { Modelling of } \\
\text { Control Char. }\end{array}$ & none & $\begin{array}{l}\text { by network load flow } \\
\text { solution process }\end{array}$ & $\begin{array}{l}\text { by conduction angle } \\
\text { adjustment scheme }\end{array}$ \\
\hline $\begin{array}{l}\text { Convergence } \\
\text { Criteria }\end{array}$ & \multicolumn{3}{|c|}{$\begin{array}{l}\text { Solution has converged when changes of the equivalent } \\
\text { harmonic current sources between two successive } \\
\text { iterations are within certain limits (typically } 10^{-4} \text { p.u.). } \\
\text { (in the case of compensator with unknown conduction } \\
\text { angles, the changes of conduction angles are also checked.) }\end{array}$} \\
\hline Model Feature & built-in element & built-in element & non-built-in element \\
\hline Comments & \multicolumn{3}{|c|}{$\begin{array}{l}\text { The TCR current source model in Chapter } 2 \text { is a special } \\
\text { case of the TCR harmonic Norton equivalent circuit model. }\end{array}$} \\
\hline
\end{tabular}




\section{Appendix E}

\section{Selected Test System Data}

There are many case studies presented in this dissertation. These cases are summarized in Table E.1. Among them, the data of two extensively tested systems and synchronous machines are listed in detail in this appendix.

1. List of test cases.

Table E.1: List of test cases.

\begin{tabular}{|c|c|c|c|c|}
\hline Chpt. & Case & Application & Ref. & Comments \\
\hline \multirow{3}{*}{2} & $\begin{array}{l}\text { Case 1: } \\
\text { TAUC-19 bus }\end{array}$ & $\begin{array}{l}\text { measurement comparison, } \\
\text { harmonic profile, } \\
\text { convergence test }\end{array}$ & {$[25]$} & data is listed \\
\hline & $\begin{array}{l}\text { Case } 2: \\
\text { TAUC- } 9 \text { bus }\end{array}$ & $\begin{array}{l}\text { harmonic profile, } \\
\text { convergence test }\end{array}$ & [25] & $\begin{array}{l}\text { modified } \\
\text { from case } 1\end{array}$ \\
\hline & $\begin{array}{l}\text { Case 3: } \\
\text { IEEE-14 bus }\end{array}$ & & {$[26]$} & modified form \\
\hline \multirow{4}{*}{3} & $\begin{array}{l}\text { Case 1: } \\
\text { B.C. Hydro } \\
\text { TLR railway }\end{array}$ & $\begin{array}{l}\text { measurement comparison, } \\
\text { unbalanced load flow, } \\
\text { frequency scan, } \\
\text { convergence test }\end{array}$ & $\begin{array}{l}{[32]} \\
{[27]} \\
{[33]}\end{array}$ & data is listed \\
\hline & Case 2: BPA & convergence test & {$[12]$} & \\
\hline & Case 3 & & {$[25]$} & modified form \\
\hline & $\begin{array}{l}\text { Case } 4: \\
\text { IEEE-118 bus }\end{array}$ & & [26] & $\begin{array}{l}\text { single-phase } \\
\text { test case }\end{array}$ \\
\hline
\end{tabular}

TAUC - TransAlta Utilities Corporation system. 
Table E.2: List of test cases (continue from previous table).

\begin{tabular}{|c|c|c|c|c|}
\hline Chpt. & Case & Application & Ref. & Comments \\
\hline \multirow[b]{2}{*}{4} & Case 1: CEMIG & measurement comparison & {$[10]$} & \\
\hline & $\begin{array}{l}\text { Case 2: } \\
\text { CEMIG modified }\end{array}$ & $\begin{array}{l}\text { EMTP comparison, } \\
\text { harmonic unbalance }\end{array}$ & {$[10]$} & \\
\hline 5 & $\begin{array}{l}\text { machine } \\
\text { test cases }\end{array}$ & $\begin{array}{l}\text { comparison with } \\
\text { EMTP \& theory, } \\
\text { harmonic profile, } \\
\text { convergence test }\end{array}$ & $\begin{array}{l}36] \\
{[37]} \\
{[38]}\end{array}$ & data is listed \\
\hline \multirow{4}{*}{6} & $\begin{array}{l}\text { A practical } \\
\text { compensator }\end{array}$ & $\begin{array}{l}\text { unbalanced } \\
\text { harmonic studies, }\end{array}$ & {$[25]$} & data is listed \\
\hline & $\begin{array}{l}\text { Case } 1: \\
\text { TAUC-19 bus }\end{array}$ & $\begin{array}{l}\text { measurement comparison, } \\
\text { harmonic profile, } \\
\text { convergence test }\end{array}$ & [25] & $\begin{array}{l}\text { data is listed, } \\
\text { three-phase } \\
\text { test case }\end{array}$ \\
\hline & $\begin{array}{l}\text { Case } 2: \\
\text { TAUC-9 bus }\end{array}$ & $\begin{array}{l}\text { harmonic profile, } \\
\text { convergence test }\end{array}$ & {$[25]$} & $\begin{array}{l}\text { modified } \\
\text { from case } 1\end{array}$ \\
\hline & $\begin{array}{l}\text { Case 3: } \\
\text { IEEE-14 bus }\end{array}$ & & {$[26]$} & $\begin{array}{l}\text { three-phase } \\
\text { test case }\end{array}$ \\
\hline
\end{tabular}

TAUC - TransAlta Utilities Corporation system.

2. List of 19 bus TransAlta Utilities Corporation system data.

Table E.3: Transformer short circuit test data.

\begin{tabular}{||l|c|c|c|c||}
\hline Name & Test winding & $V_{\text {rate }}(\mathrm{KV})$ & $S_{\text {rate }}(\mathrm{MVA})$ & Value (pu\%) \\
\hline \hline Compensator & $\mathrm{H}-\mathrm{Y}$ & 240.0 & 250.0 & 9.53 \\
transformer & $\mathrm{H}-\Delta$ & 240.0 & 250.0 & 9.43 \\
& $\mathrm{Y}-\Delta$ & 20.0 & 125.0 & 15.3 \\
\hline LDN & $\mathrm{H}-\mathrm{L}$ & 500.0 & 400.0 & 11.85 \\
transformer & $\mathrm{H}-\mathrm{T}$ & 500.0 & 400.0 & 76.95 \\
& $\mathrm{~L}-\mathrm{T}$ & 240.0 & .400 .0 & 61.07 \\
\hline
\end{tabular}


Table E.4: Transmission line and equivalent source data.

\begin{tabular}{|c|c|c|c|c|c|c|}
\hline Bus-I & Bus-J & $\begin{array}{r}R_{1} \\
\text { Ohm }\end{array}$ & $\begin{array}{r}X_{1} \\
\text { Ohm }\end{array}$ & $\begin{array}{r}B \\
\mu \text { Mho }\end{array}$ & $\begin{array}{r}R_{0} \\
\text { Ohm }\end{array}$ & $\begin{array}{r}X_{0} \\
\text { Ohm }\end{array}$ \\
\hline \multicolumn{7}{|c|}{ Transmission line data } \\
\hline BENA & R.DE & 1.846 & 11.306 & 131.437 & 10.262 & 39.918 \\
\hline R.DE & CROS & 5.574 & 34.142 & 396.916 & 2.560 & 15.871 \\
\hline CROS & JANE & 3.136 & 22.324 & 206.503 & 18.816 & 0.267 \\
\hline BENA & SARC & 9.770 & 56.200 & 616.650 & 49.687 & 191.777 \\
\hline JANE & PEIG & 11.41 & 63.179 & 678.093 & 55.998 & 215.451 \\
\hline R.DE & NEVI & 4.604 & 23.953 & 274.106 & 22.371 & 3.683 \\
\hline SARC & E.CA & 0.870 & 5.871 & .535 & 3.954 & 7.495 \\
\hline E.CA & JANE & 0.660 & 5.602 & 39.144 & 3.663 & 6.875 \\
\hline BENA & JANE & 10.955 & 69.196 & 745.055 & 61.388 & 31.148 \\
\hline W.BR & LETH & 9.839 & 63.149 & 614.901 & 48.861 & 213.104 \\
\hline LANG & W.BR & 11.461 & 64.288 & 740.733 & 5.688 & 30.012 \\
\hline R.DE & JANE & 532 & 55.824 & .441 & 0.788 & 37.278 \\
\hline BENA & SARC & 8.689 & 56.184 & 243 & 0.065 & 0.352 \\
\hline R.DE & JANE & 9.529 & 55.803 & 248 & .475 & 188.293 \\
\hline W.BR & $3 \mathrm{~A}$ & 2.284 & 13.374 & 173 & .397 & 8.612 \\
\hline $933 \mathrm{~A}$ & ANDE & 100 & 23.766 & 953 & .492 & 7.122 \\
\hline W.BR & $4 \mathrm{~A}$ & 284 & 13.374 & 173 & 397 & 8.612 \\
\hline $934 \mathrm{~A}$ & ANDE & 08 & 23.766 & 953 & 492 & 7.122 \\
\hline $933 \mathrm{~A}$ & DME & 28 & 56.923 & 457 & .519 & 206.979 \\
\hline $934 \mathrm{~A}$ & JENN & 93 & 26.881 & 077 & 894 & 7.681 \\
\hline JENN & DOME & 132 & 30.021 & 228 & 25.602 & 109.174 \\
\hline JENA & LANG & 58 & 8.354 & 002 & 5.468 & 5.168 \\
\hline JENA & LANG & 0.758 & 8.354 & 002 & 468 & 5.168 \\
\hline NEVI & CORD & 4.666 & 24.250 & 285.141 & 100 & 5.824 \\
\hline ANDE & CORD & 34 & 48.960 & & 381 & 31.44 \\
\hline ALX & LDN & 7.924 & $100: 64$ & 1605.09 & 098 & 3.11 \\
\hline \multicolumn{7}{|c|}{ Equivalent source data } \\
\hline \multicolumn{2}{|c|}{ BENA } & 1.91 & 16.16 & & 9.094 & 39.152 \\
\hline \multicolumn{2}{|c|}{ R.DE } & 3.929 & 28.18 & & 14.472 & 62.272 \\
\hline \multicolumn{2}{|c|}{ CORD } & 1.545 & 31.11 & & 0.921 & 24.106 \\
\hline
\end{tabular}


Table E.5: Bus load conditions.

\begin{tabular}{||c|c|c||}
\hline Bus & Active Power (MW) & Reactive Power (MVar) \\
\hline \hline DOME & 76.3 & 0.0 \\
\hline CROS & 81.0 & 17.7 \\
\hline W.BR & 90.0 & 66.5 \\
\hline NEVI & 45.0 & -2.7 \\
\hline LETH & 87.5 & 49.2 \\
\hline PEIG & 95.7 & 11.8 \\
\hline JANE & 419.0 & 19.5 \\
\hline E.CA & 151.0 & -23.8 \\
\hline SARC & 371.0 & -40.1 \\
\hline ALX & -28.0 & 98.0 \\
\hline BENA & \multicolumn{3}{|c||}{ Slack bus } \\
\hline R.DE & \multicolumn{2}{|c||}{ Slack bus } \\
\hline CORD & \multicolumn{2}{|c||}{ Slack bus } \\
\hline
\end{tabular}

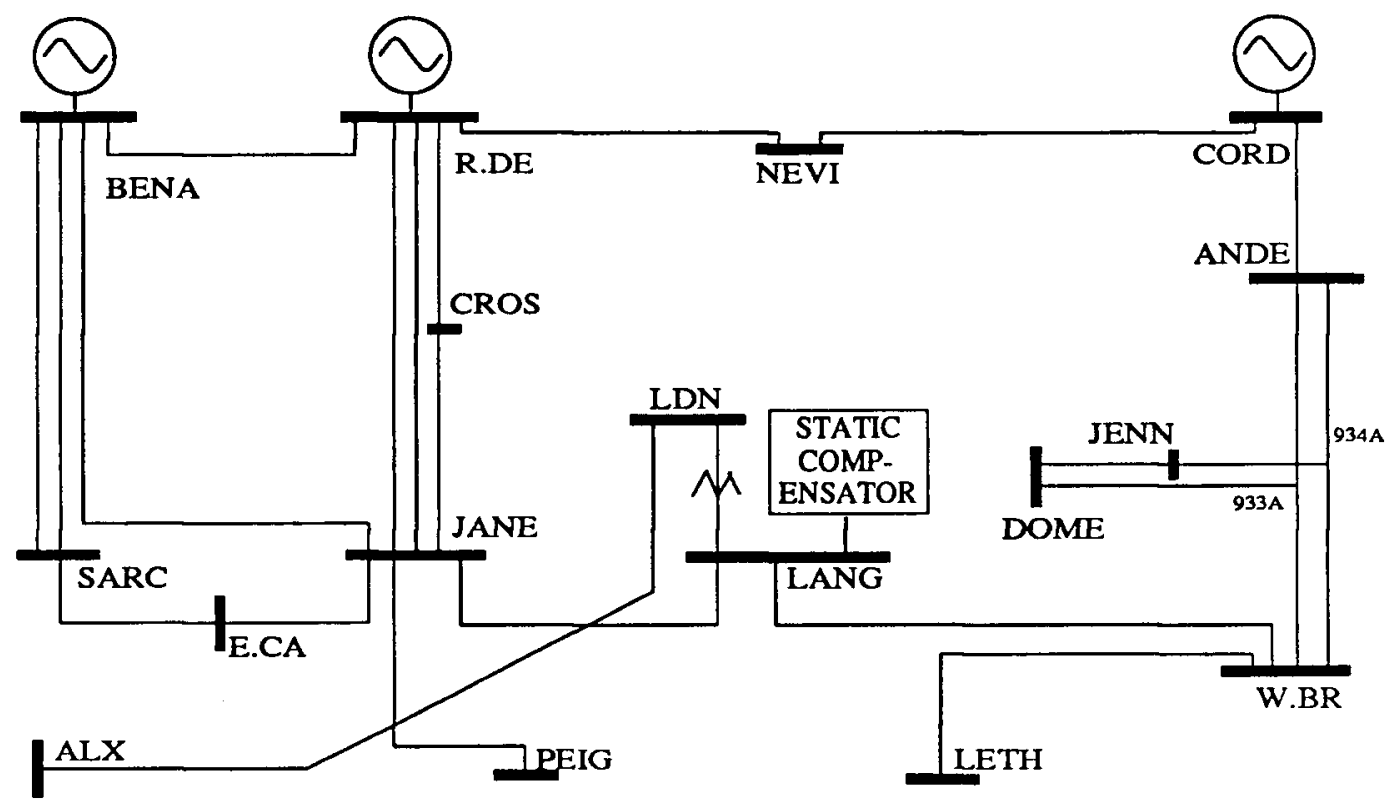

Figure E.1: 19 bus TransAlta Utilities Corporation test system. 
Table E.6: Data of static compensator components and filters.

\begin{tabular}{||l|c|c|c||}
\hline Device & Reactor $(\mathrm{mH})$ & Capacitor $(\mu \mathrm{F})$ & Resistor $(\mathrm{Ohm})$ \\
\hline Shunt & 6.1405 & 178.4 & \\
\hline TSC SRG & 1.959 & & 33.0 \\
\hline 11th & 1.456 & 39.47 & \\
\hline 13th & 0.892 & 46.16 & 88.0 \\
\hline 23rd & 0.159 & 82.74 & 14.0 \\
\hline
\end{tabular}

3. List of machine data.

Table E.7: Machine data in per-unit.

\begin{tabular}{||l|lllll||}
\hline parameter & \multicolumn{5}{|c||}{ value } \\
\hline machine \# & 1 & 2 & 3 & 4 & 5 \\
\hline$L_{d}$ & 1.0 & 1.4 & 1.0 & 1.505 & 1.2 \\
$M_{d f}$ & 0.8 & 1.3 & 0.8 & 1.365 & 1.0 \\
$L_{f f}$ & 1.1 & 2.0 & 1.0 & 1.505 & 1.2 \\
$L_{D D}$ & 0.9 & 1.34 & 0.95 & 1.405 & 1.0 \\
$L_{q}$ & 0.8 & 0.7 & 0.6 & 1.368 & 0.8 \\
$M_{q g}$ & 0.6 & 0.6 & 0.4 & 1.275 & 0.6 \\
$L_{g g}$ & 1.0 & 1.6 & 0.7 & 1.368 & 0.831 \\
$L_{o}$ & 0.08 & 0.08 & - & 0.01 & - \\
$R_{a}$ & 0.05 & 0.05 & 0.001 & 0.03 & 0.005 \\
$R_{f}$ & 0.2 & 0.08 & 0.004 & 0.00086 & 0.0005 \\
$R_{D}$ & 0.01 & 0.06 & 0.02 & 0.0056 & 0.02 \\
$R_{g}$ & 0.01 & 0.08 & 0.04 & 0.0072 & 0.02 \\
\hline Note: $\quad M_{d f}=M_{d D}=M_{f D}$ \\
\hline \multicolumn{7}{|c|}{} \\
\hline
\end{tabular}

4. List of B.C. Hydro Tumbler Ridge area system data. 


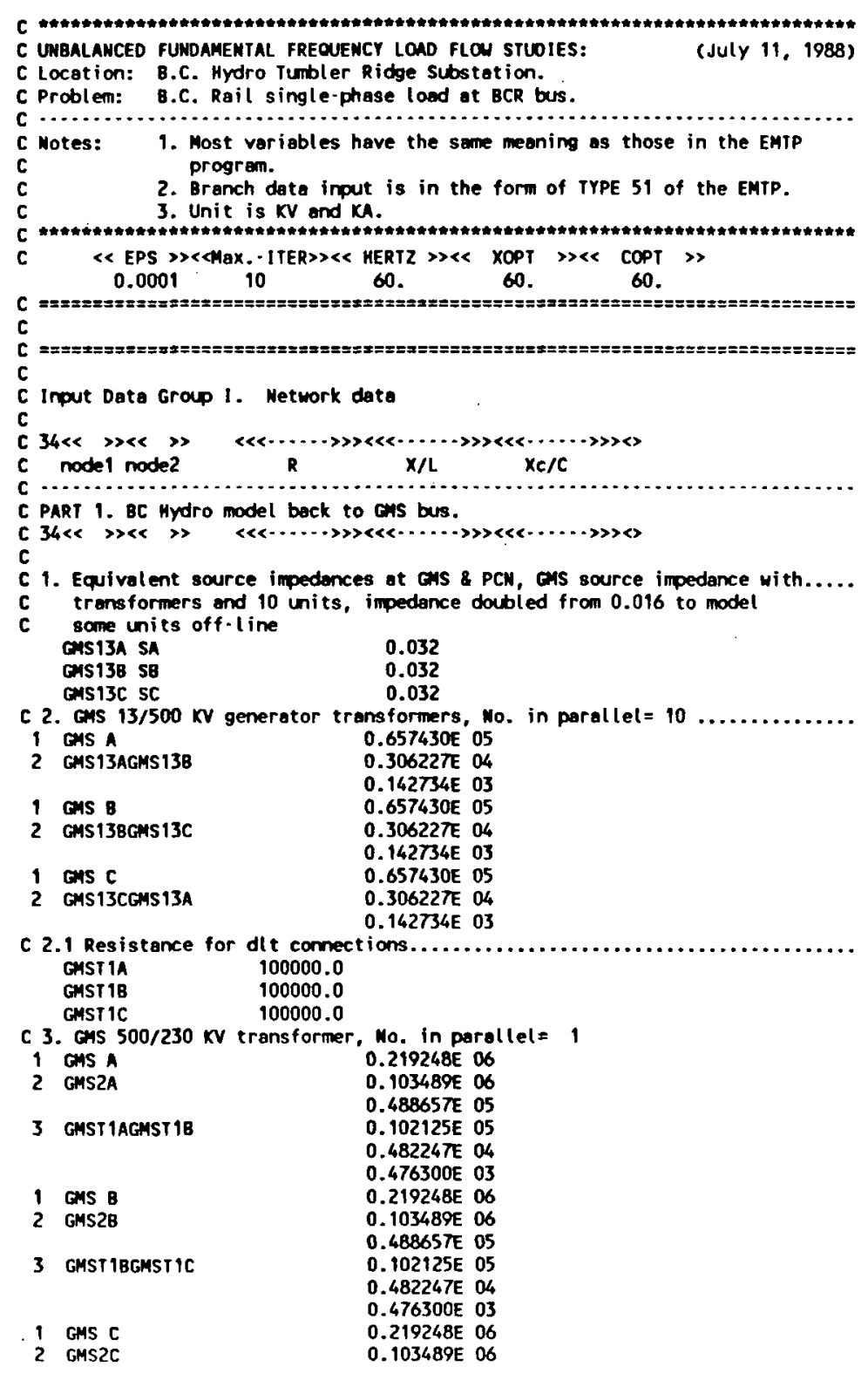

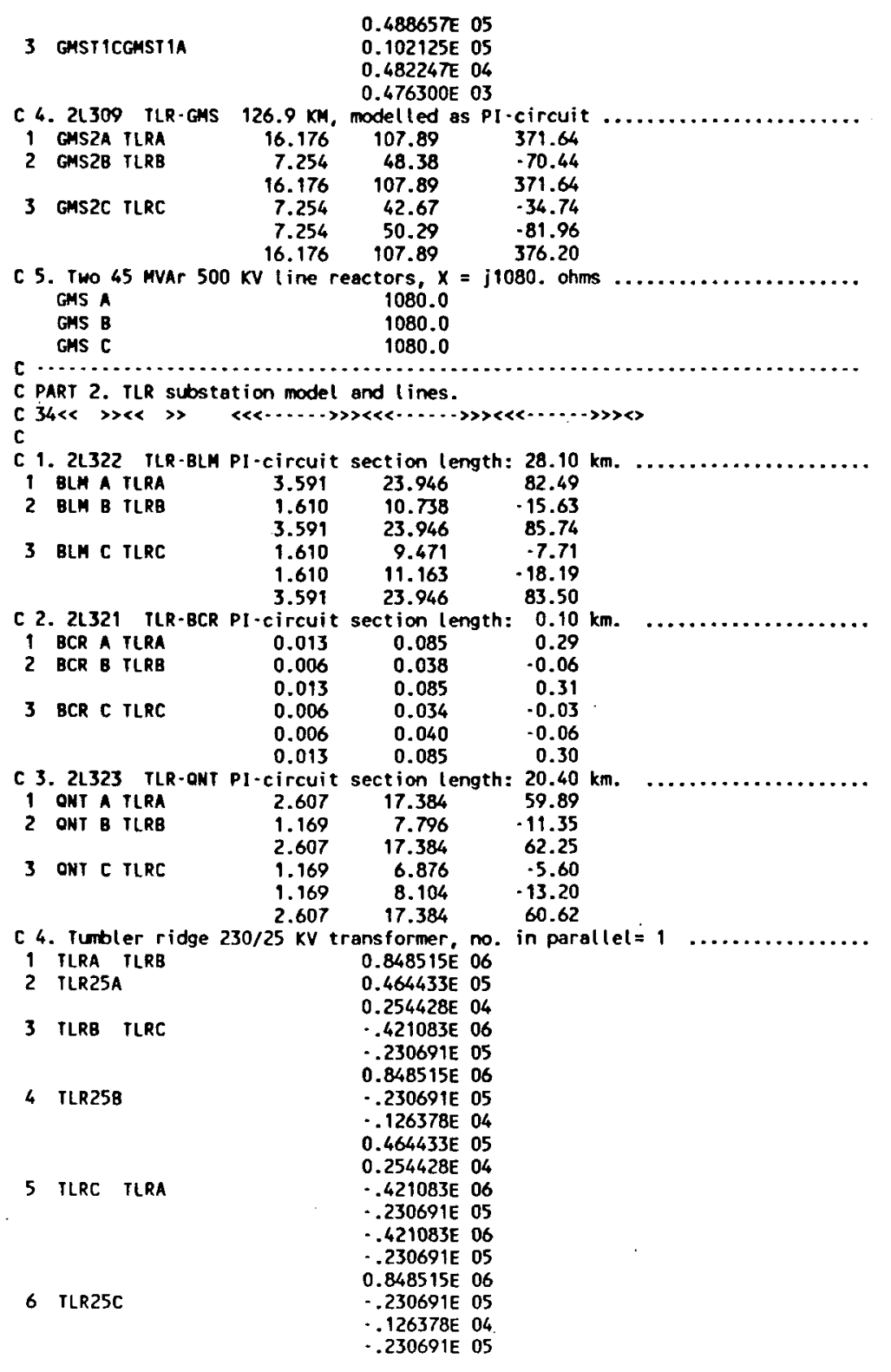




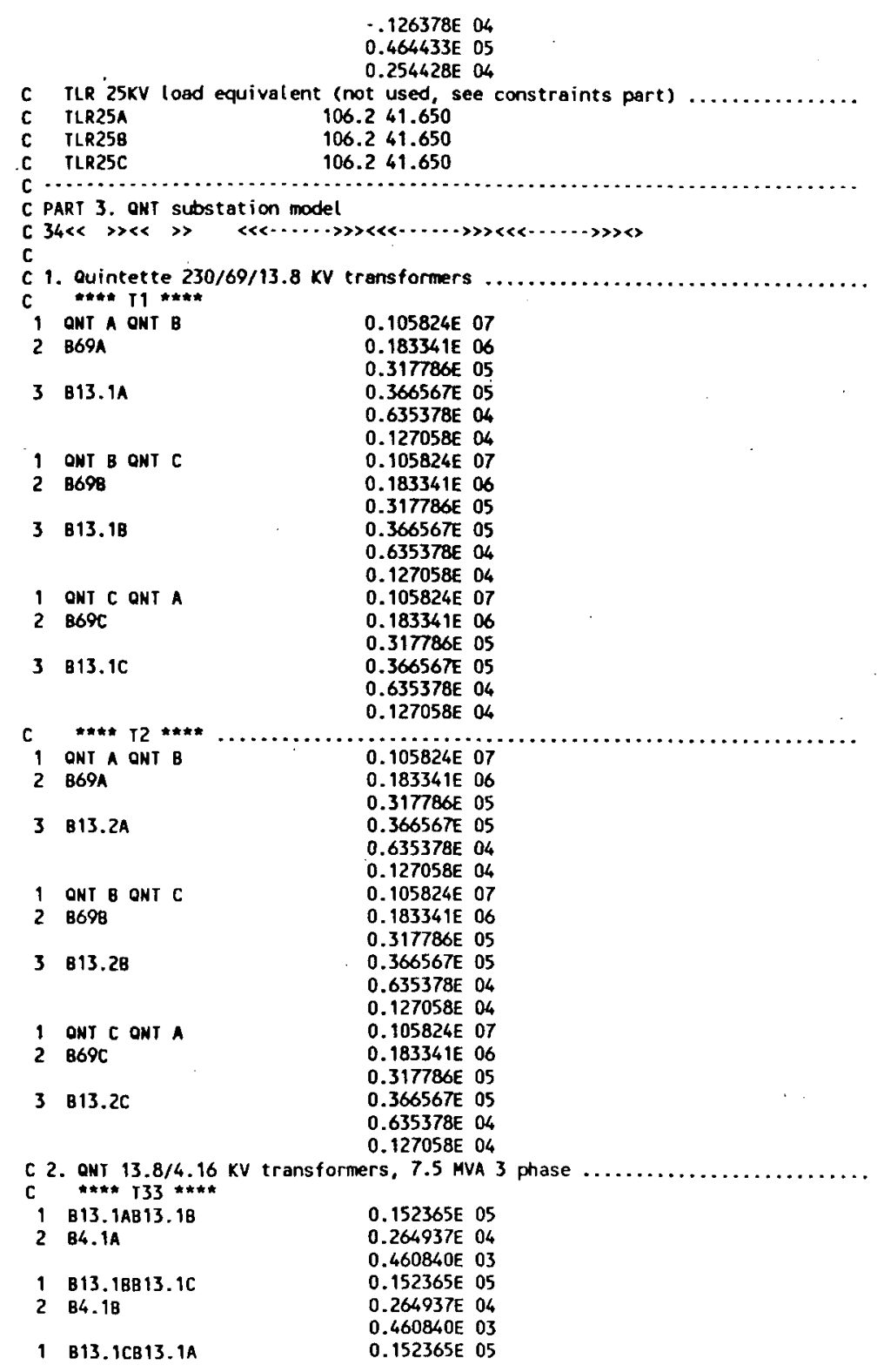

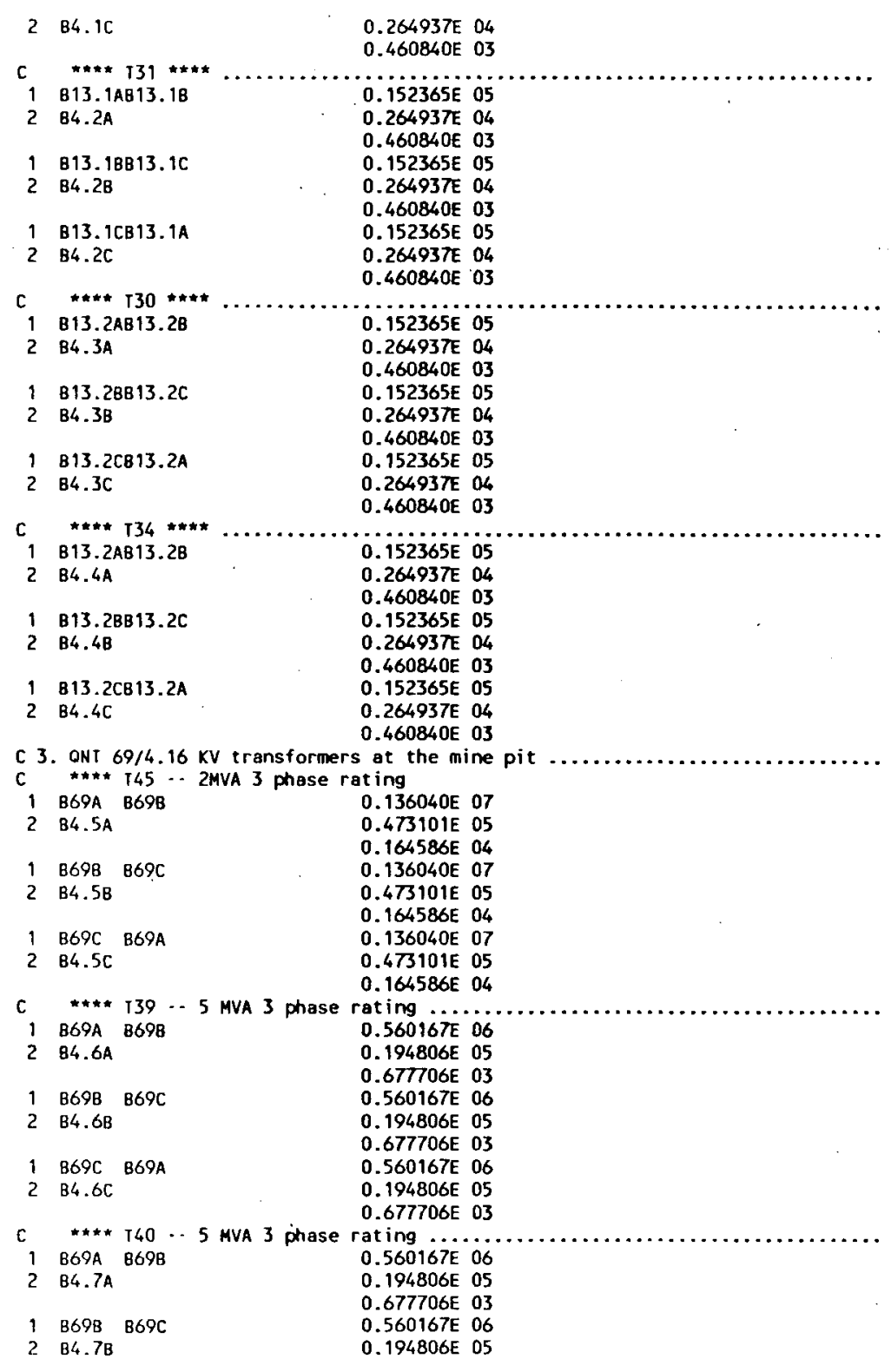




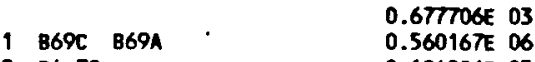 \\ 2 B4.7C $0.194806 E 05$ \\ 4. Motor shunt capacitors 0.677 rot}

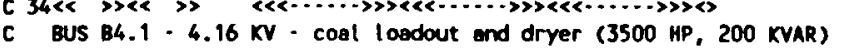

B4.1A 11557.

$\begin{array}{ll}\text { B4.18 } & 11557 . \\ \text { B4.1C } & 11557 .\end{array}$

C BUS B4.2- $4.16 \mathrm{kV}$ - preparation plant and thickener (2300 MP, 550 KVAR)

$34.2 A$

$84.2 \mathrm{C}$

C BUS $84.3-4.16 \mathrm{kV}$ - preparation plont (6600 MP, $1500 \mathrm{KVAR})$

$84.31 .4 .16 \mathrm{kN}-$ preparation plont 866067

34.38 86677

C BUS 84.4 - $4.16 \mathrm{KV} \cdot$ dryer \& elean cool (5700 HP, $125 \mathrm{KVAR})$

$84.4 \mathrm{~A}$
$\mathrm{BL} .4 \mathrm{~B}$

B4.4B

7223 . B4.5A 8668. $\begin{array}{ll}\text { B4.5B } & 8668 . \\ 84.5 \mathrm{C} & 8668 .\end{array}$

C BUS $84.6 \cdot 4.16 \mathrm{kV}$ - pit conveyor oreo (1100 HP, 150 KVAR ossumed)

84.61. 8668 .

$\begin{array}{ll}84.68 & 8668 . \\ 84.6 C & 8668 .\end{array}$

C BUS B4.7-4.16 kV - pit conveyor orea (400 HP, 75 KVAR)

84.7A 4334 .

$\begin{array}{ll}84.7 \mathrm{~B} & 4334 . \\ 84.7 \mathrm{C} & 4334 .\end{array}$

C PART 4. BLM substation

C PART 4. BLM substation

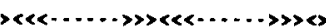

C Bullmoose $230 / 25 \mathrm{kV}$ transformers, no. in parallel= 2

1 BLM A BLM B

$0.848515 E$ OS

$0.530924 E$ OS
$0.332405 E$ O4

3 BLM B BLM C

$\because .421083 E$ O6

$0.848515 E$ OS

$.263637 \mathrm{E} 05$

$0.530924 \mathrm{E}$ OS

4 BL.M258

$0.332405 E 04$

$.421083 E 06$

.263637 OS

.421083E 06

-.263637 E 05

$0.848515 E 06$

6 BLM25C
$0.332405 E 04$

C END OF METHORK DATA

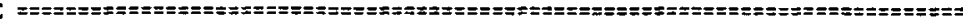

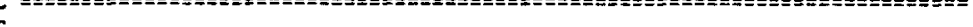

C Input Data Group 11. Constraints data

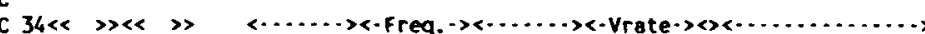

$c$ nodel node2 P/Mag. freq. O/Ang. Vrated P" load character

C Note: load types are identified as

$P Q \ldots$ constant $P Q$ lood. $P *=1$, single-phase. $P \#=1=1$, type-3.

op .... mult iphase motor load (ingut value < 0 means comsumption).

sK .... slack machine.

PV ... PV machine.

cS... current source.

vs $\ldots$ vol tage source.

c $\ldots$. vs $\ldots$ voltage source.

C 1. Source at GMS: essume 13.0/SoRT(3), keep TLR voltage normal

$\begin{array}{lllll}\text { VS } S A & 8.30 & 60.0 & 0.0 & 1 \\ \text { VS } & 8 B & 8.30 & 60.0 & -120.0\end{array}$

$\begin{array}{llll}\text { VS SB } & 8.30 & 60.0 & -120.0 \\ \text { YS } & 8.30 & 60.0 & -240.0\end{array}$

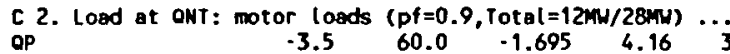

$\begin{array}{lll}B 4.1 A \text { B4.1B } & 0.0989 & 0.9843 \\ \text { B4.18 B4.1C } & 0.0247 & 0.2469\end{array}$

$\begin{array}{ll}0.0247 & 0.2461 \\ 0.0989 & 0.9843\end{array}$

$\begin{array}{lll}84.9 C 84.11 & 0.0247 & 0.2461 \\ & 0.0247 & 0.2461\end{array}$

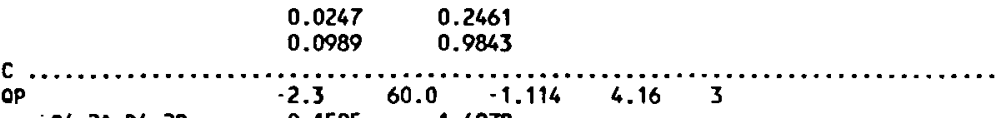

$\begin{array}{lll}B 4.2 A B 4.28 & 0.1505 & 1.4978 \\ B 4.2 B \text { B } 4.2 C & 0.0376 & 0.3745\end{array}$

$\begin{array}{lll}0.1505 & 1.4978 \\ & 0.036 & 0.375\end{array}$

B4.2C B4.2A $\quad 0.0376 \quad 0.3745$

$\begin{array}{rr}0.0376 & 0.3745 \\ 0.1505 & 1.4978\end{array}$

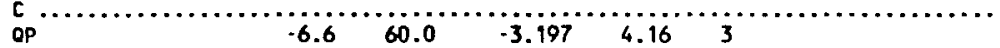

$\begin{array}{lll}84.3 A \text { B4.3B } & 0.0524 & 0.5520 \\ 84.38 B 4.3 C & 0.0131 & 0.1305\end{array}$

$\begin{array}{lll}84.3 C \text { B } 4.3 \mathrm{~A} & 0.0524 & 0.5520 \\ & 0.0131 & 0.1305\end{array}$

$\begin{array}{rrr}0.0131 & 0.1305 \\ 0.0524 & 0.5520\end{array}$

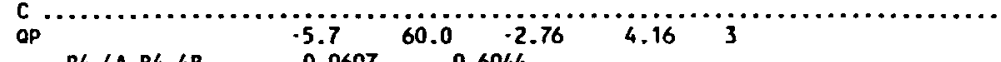

$\begin{array}{lll}B 4.4 A B 4.4 B & 0.0607 & 0.6044 \\ B 4.4 B ~ B 4.4 C & 0.0152 & 0.551\end{array}$

$\begin{array}{ll}0.0152 & 0.1511 \\ 0.0607 & 0.6044\end{array}$

$\begin{array}{lll}B 4.4 C B 4.4 \mathrm{~A} & 0.0152 & 0.1511\end{array}$

$\begin{array}{ll}0.0152 & 0.1511 \\ 0.0607 & 0.6044\end{array}$


OP

\begin{tabular}{|c|c|c|}
\hline & .1 .05 & 60.0 \\
\hline $\begin{array}{l}84.5 A \quad B 4.5 B \\
B 4.5 B \quad B 4.5 C\end{array}$ & $\begin{array}{l}0.3296 \\
0.0824 \\
0.3296\end{array}$ & $\begin{array}{l}3.2810 \\
0.8202 \\
3.2810\end{array}$ \\
\hline $84.5 C B 4.5 \mathrm{~A}$ & $\begin{array}{l}0.0824 \\
0.0824 \\
0.3296\end{array}$ & $\begin{array}{l}0.8202 \\
0.8202 \\
3.2810\end{array}$ \\
\hline
\end{tabular}

c... Looding too small compering with $\times$ former rating (assume 2 in parallel) ...

$04.6484 .68 \quad-2.200 .1573 \quad 1.5659$

$\begin{array}{lll}0.0394 & 0.3915 \\ 0.1573 & 1.5659\end{array}$

$84.6 C 84.64 \quad 0.0394 \quad 0.3915$

$0.0394 \quad 0.3915$

C... Laeding increased $(0.4 * 5$ in parollel) since xformer rating 5AVA

op $\quad 2.0 \quad 60.0 \quad-0.194 \quad 4.16 \quad 3$

B4.7A B4.78 $\quad 0.2880 \quad 1.7178$

$\begin{array}{lll} & 0.0721 & 0.5294 \\ & 0.2880 & 1.7178\end{array}$

$84.7 \mathrm{C} 84.7 \mathrm{~A} \quad 0.0729 \quad 0.5294$

$0.0721 \quad 0.529$

0.2880

c 3 . Load at BLM: motor loads + individual load (total $=8-9$ 9M)

(6)

$c$....... lood increased as individual laods

$\begin{array}{lllll} & -2.0 & -0.8 & 25.0 & 3\end{array}$

$\begin{array}{lll}\text { BLM25ABLM25B } & 9.333 & 62.213 \\ \text { BLM25BBLL25C } & 2.333 & 15.553\end{array}$

$2.333 \quad 15.553$

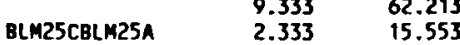

$\begin{array}{ll}2.333 & 15.553 \\ 2.333 & 15.553 \\ 9.333 & 62.213\end{array}$

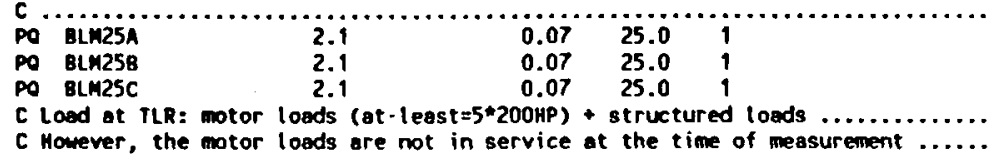

c The use of these motors will generally reduce voltage unbalance ...............

c OP TLR25ATLR25B $\quad-1.0 \quad 28.98 \quad 126.0903$

$\begin{array}{llrr}\text { C } & \text { TLR25ATLR25B } & 28.98 & 124.09 \\ \text { C } & \text { TLR25BTLR25C } & 7.25 & 31.02 \\ \text { C } & & 28.98 & 124.09\end{array}$

$\begin{array}{lrr}\text { TLR25CTLR25A } & 28.98 & 124.09 \\ & 7.25 & 31.02\end{array}$

$\begin{array}{rrr}\text { C } & 7.25 & 31.02 \\ \text { C } & 28.98 & 124.09\end{array}$

C 4. Toun loods, pf correction assumed $0.7 \quad 25.0$.

PO TL25A

TLR25B

TLR25C

C Loed of B.C Rail: assume 4 4.5mw/GHVA. pf -0.8

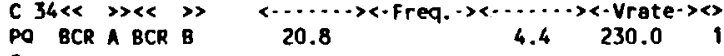

20.8

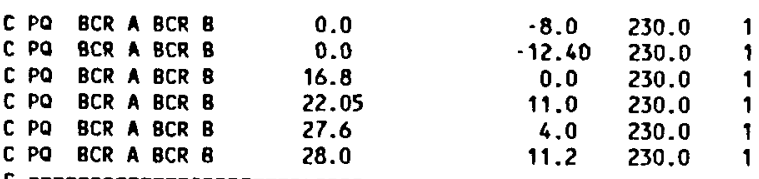

C PO BCR A BCR B 28.0

C END OF CONSTRAINTS DAIA

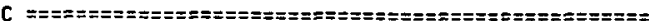




\section{PUBLICATIONS}

Xu, W., J.R. Marti and H.W. Dommel, "Harmonic Analysis of Systems with Static Compensators", Accepted for publication on IEEE Trans. on Power Systems.

$X u$, W., J.R. Marti and H.W. Dommel, "A Multiphase Harmonic Load Flow Solution Technique", Accepted for publication on IEEE Trans. on Power Systems.

XU, W. and H.W. Dommel, "Computation of Steady-State Harmonics of Static Var Compensators", Proc. of the Third International Conference on Harmonics in Power Systems, Nashville, IN, Oct 1988, pp. 239-245.

Xu, W. and Q. Lu, "A New Stabilizer Design Technique for Multimachine Power Systems", Electric Power System Research (Switzerland), vol. 15, no. 2, Oct 1988, pp. 89-97.

Yu, Y., W. Xu, Q. Lu, G.K.F. Lee and J. Gao, "Decentralized Stabilizers for Large Electric Power Systems", IMACS 1988 12th World Congress on Scientific Computation Proceedings, Paris, July 1988, vol. 1, pp. 270-273.

Sachdev, M.S. and W. Xu, "A Second Order Sensitivity Load Flow Technique", Transactions on Engineering and Operating Division, CEA, vol. 26, part 3, 1987. 